\title{
SIMULATION-ENHANCED FRACTURE DETECTION: RESEARCH AND DEMONSTRATION IN U.S. BASINS
}

\author{
Phase II Final Report 2004
}

August 3, 2000 - November 30, 2004

Principal Investigator, Peter J. Ortoleva

January 2005

DOE Contract \# DE-AC26-00NT40689

Laboratory for Computational Geodynamics

Chemistry Building, Suite 203

Indiana University

Bloomington, Indiana 47405

812.855.2717 Phone

812.855.8300 Fax

ortoleva@indiana.edu 


\section{Disclaimer}

This report was prepared as an account of work sponsored by an agency of the United States Government. Neither the United States Government nor any agency thereof, nor any of their employees, makes any warranty, express or implied, or assumes any legal liability or responsibility for the accuracy, completeness, or usefulness of any information, apparatus, product, or processes disclosed, or represents that its use would not infringe privately owned rights. Reference herein to any specific commercial product, process, or service by trade name, trademark, manufacturer, or otherwise does not necessarily constitute or imply its endorsement, recommendation, or favoring by the United States Government or any agency thereof. The views and opinions of authors expressed herein do not necessarily state or reflect those of the United States Government or any agency thereof. 


\section{Abstract}

Remote detection and characterization of fractured reservoirs is facilitated in this project by developing a revolutionary software system. The Model-Automated Geo-Informatics (MAGI) software integrates basin modeling, seismic data, synthetic seismic wave propagation and well data via information theory. The result is a seismic inversion cast in terms of fracture and other reservoir characteristics. The MAGI software was fully tested on synthetic data to verify program accuracy and robustness to data error.

In Phase II, we

- collected geological information (stratigraphic, structural, thermal, geochemical, fracturing and other information across the study area (Task 4.1);

- created a GIS database that is compatible with the input requirements of MAGI (Task 4.1);

- implemented a web-based interface for user friendly access (Task 4.2);

- gathered and preprocessed seismic data for input into MAGI;

- developed two- and three-dimensional wave propagation simulators (in time domain) for fluid saturated porous media and implemented matching layer methodology for absorbing boundary conditions (Task 4.3);

- developed parallel version of the seismic simulators (Task 4.3);

- proposed an information theory framework that allows for the integration of multiple data types of a range of quality (Task 4.4);

- developed and implemented highly efficient, parallel, Gauss-Newton seismic waveform inversion code based on reciprocity theorem (Task 4.5)

- verified and demonstrated the accuracy and efficiency of the wave propagation and seismic waveform inversion codes (Tasks 4.3 and 4.5 ); and

- identified the requirements for seismic data to allow seismic inversion (Task 4.6). With these accomplishments, we are prepared to carry out a demonstration in the Illinois Basin. A database of the proposed study area and the web-based system to facilitate geologic and seismic data input are ready for this demonstration as are mapping tools for comparison and observations. 


\section{Table of Contents}

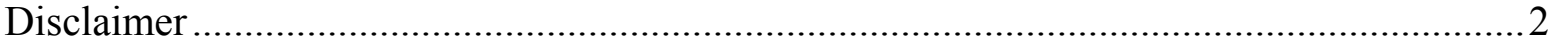

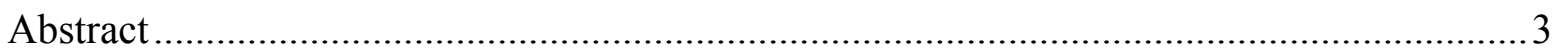

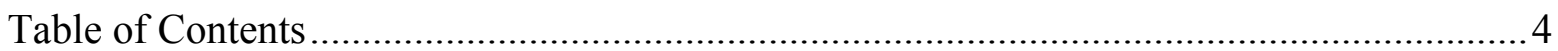

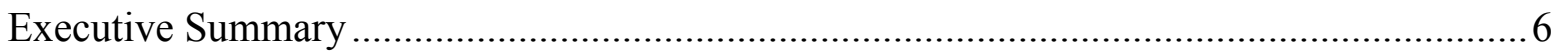

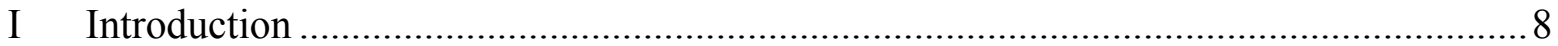

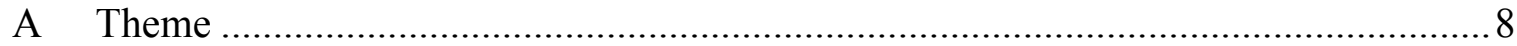

B Meeting the Phase II Plan of Work .................................................................... 13

C Remote Detection of Reservoir Properties Achieved Through the MAGI System ... 13

II Review, Quality Screen and Organize Data for Test Cases (Subtask 4.1).................. 14

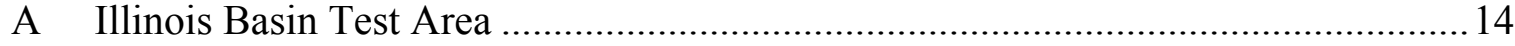

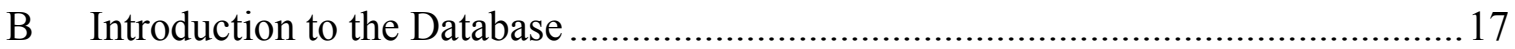

C Instructions for Using the Database ................................................................. 18

III Implement the Web-Based User Interface (Subtask 4.2) .........................................27

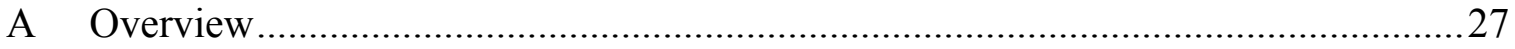

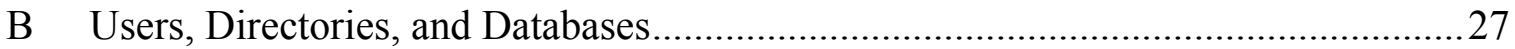

C Setting up for a Run ...................................................................................2 29

1 The Project File............................................................................................ 29

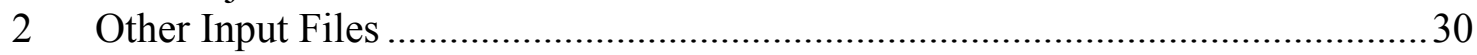

3 The Session File .......................................................................................... 30

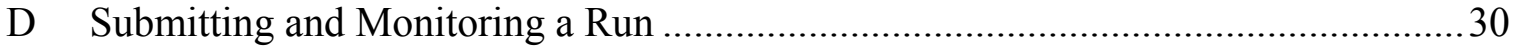

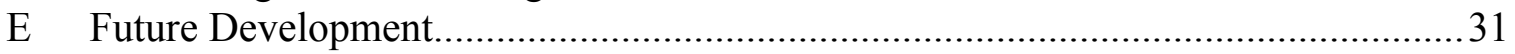

IV Develop Efficient Seismic Simulator (Subtask 4.3) ............................................... 33

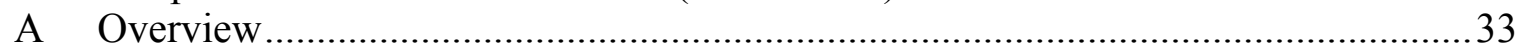

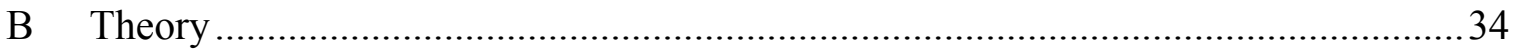

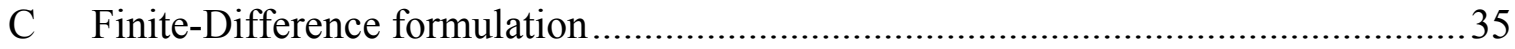

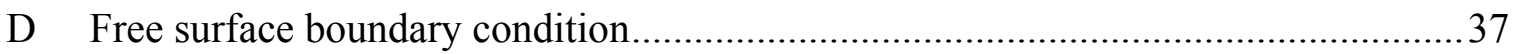

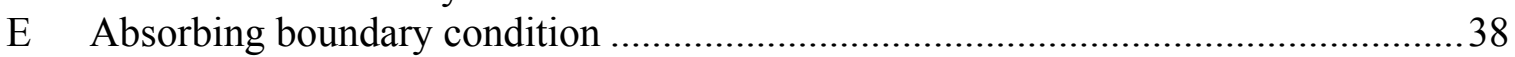

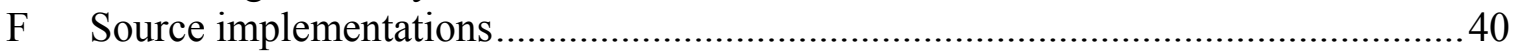

G Numerical examples: Homogeneous model ................................................ 40

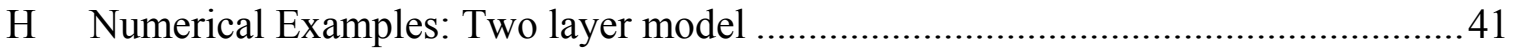

I Numerical Examples: Transition layer model ..................................................42

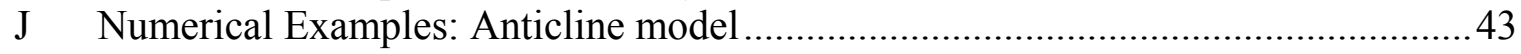

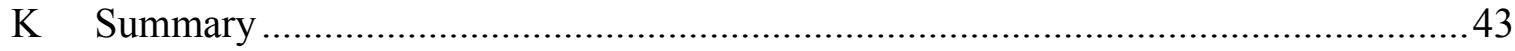

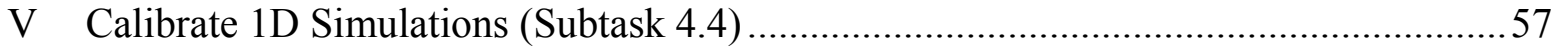

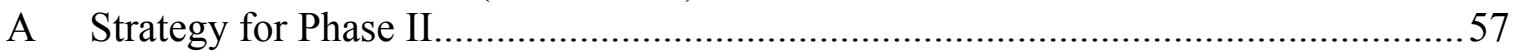

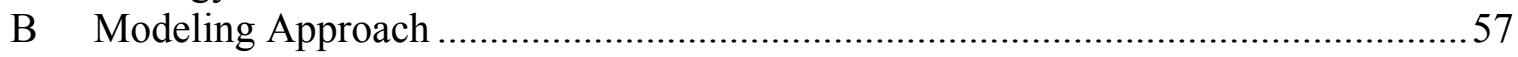

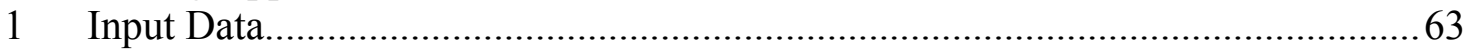

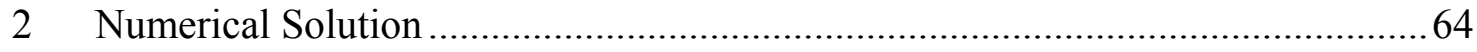

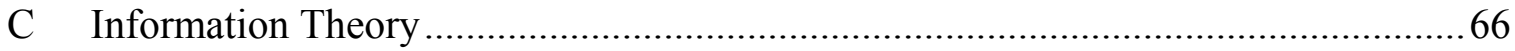

VI Develop Seismic Inversion Code Using Reciprocity Principle (Subtask 4.5) ............. 72

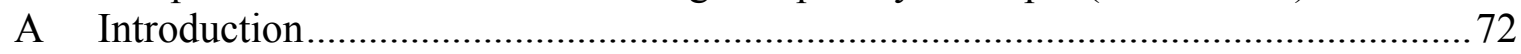

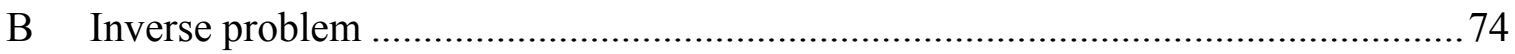

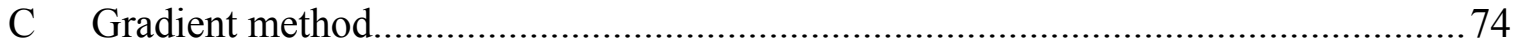

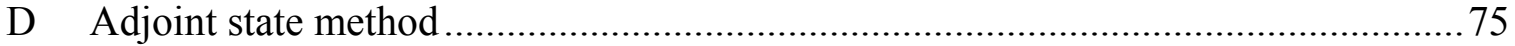


E Newton method \& Gauss-Newton method ........................................................76

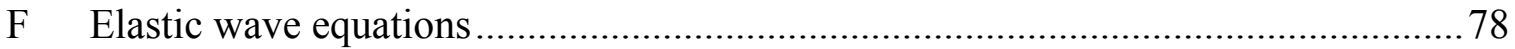

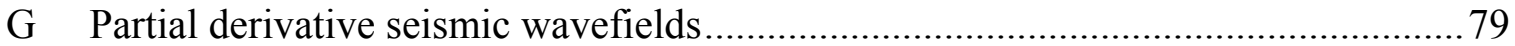

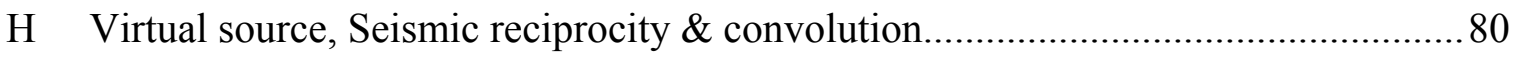

I Gradient \& Gauss-Newton method with reciprocity \& convolution ............................82 82

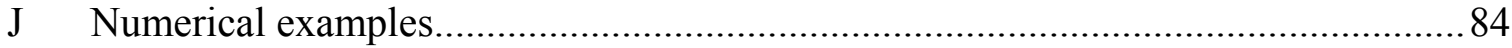

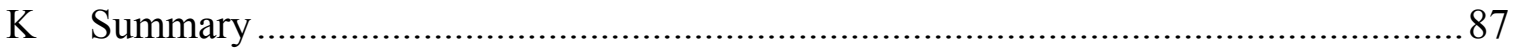

VII Compare Predicted and Observed Fracture Network Properties (Subtask 4.6) .............99

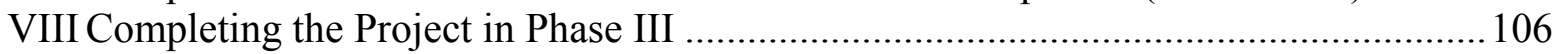

Task 5 Demonstrate SEFD in the Laconia Field of Central Harrison County ............ 106

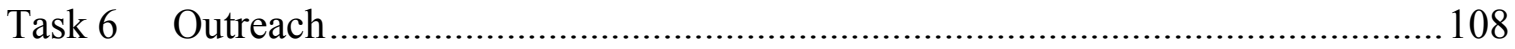

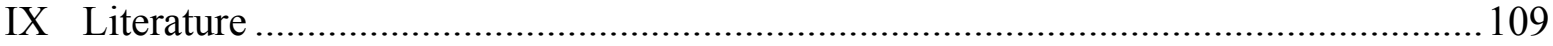

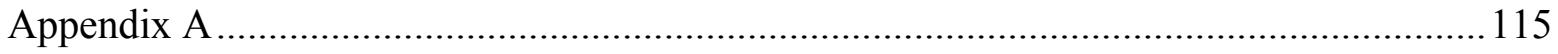

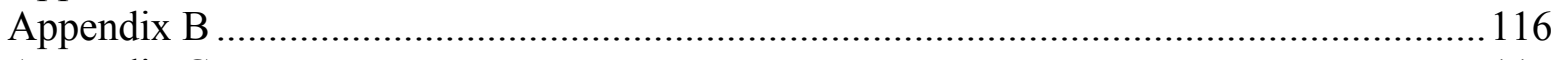

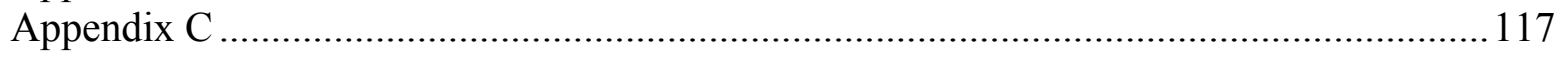

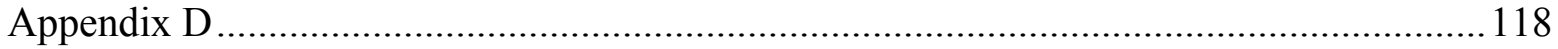




\section{Executive Summary}

This SEFD project ("Simulation-Enhanced Fracture Detection," Contract \#DE-AC2600NT40689) is a three-phase effort to develop, implement, test and demonstrate a novel strategy for characterizing the state of fractured reservoirs in the subsurface via remote technologies. This report summarizes Phase II accomplishments and concludes with recommendations for the final (Phase III) effort.

In our SEFD approach, computer simulation of the evolution of the subsurface over geological or engineering time scales is used to enhance the inversion of remote data (e.g. seismic reflections) and arrive at more refined physical images (e.g. fracture characteristics and other reservoir properties) than the more typical display of seismic velocity anomalies. Difficulties in basin modeling are overcome by model/data integration, while the model facilitates the interpretation of remote data. In this way, basin modeling and seismic inversion become one seamless activity, with the strengths of one compensating for the weaknesses of the other.

This project arose out of a need to integrate a number of approaches. Our strategy is to develop a computational framework to realize and automate this integration. Our vision starts with recognition that the industry must address the great uncertainty we have in the state of the subsurface. Rather than view this uncertainty as a barrier, we use it as the starting point of our approach. A classic measure of the uncertainty in the state of a system is the entropy. Our objective is to construct the probability by admitting maximum uncertainty that could be present in light of what is actually known - i.e. seismic, well log, production and other data. In this way, we arrive at an objective assessment of uncertainty. We have developed this general approach, using it to construct the most probable state of the subsurface in light of the available data. This framework yields an automatable algorithm for constructing the subsurface state.

The difficulty is that seismic data is only indirectly related to the spatial distribution of fracturing, porosity, gas versus water or oil saturation, and a myriad of other seismic wave velocity-altering factors. Similarly we address the difficulties in extrapolating information away from the well and interpolating between wells.

Achievement of our goal required the following technical advances:

- two- and three-dimensional seismic wave propagation programs were written that are major advances over pre-existing software due to their ability to simulate wave propagation in a fluid-saturated poroelastic medium with absorbing boundary conditions at the computational boundaries to avoid artifacts to arrive at the most efficient simulator available;

- an information theory framework that allows for the integration of multiple data types of a range of quality;

- a seismic waveform inversion approach based on the reciprocity theorem and a regularization approach for noise reduction;

- a MAGI user interface for input manipulation and archiving of user information about a study area;

- a more computationally efficient one-dimensional basin RTM simulator;

- a relational database of geological information that is compatible with the input requirements of our MAGI system and has the requisite stratigraphic, structural, thermal, 
geochemical, fracturing and other information across the Harrison County, Indiana, study area; and

- seismic data was gathered and preprocessed for input into our software system and chosen specifically to allow us to carry out our test for Phase II.

All the above software and database were written/collated by us constituting an extraordinary accomplishment that in many ways exceeded the original project scope but which was necessary to accomplish our goals.

In particular, our seismic waveform inversion methodology has the following special features. Most geophysical inverse problems have been solved by linearized iterative inversion. The forward simulation is approximated by a set of equations linearized about a reference model and a solution of the resulting linearized inverse problem is computed. Then the solution is used as a new reference model for the next step and the process is repeated until convergence. For seismic waveform inverse problems, Newton-type second order methods are computationally too expensive to be practical. Instead, seismic waveform inversion has been accomplished by the gradient method (Lailly 1983; Tarantola 1984) wherein it was shown that the adjoint state method for constructing the gradient direction for the inversion of the acoustic problem could be determined without computing the partial derivatives explicitly. Newton-type seismic waveform inversion requires huge amounts of memory and computation, far greater than that of a typical scientific workstation. In order to overcome this computer resource limitation, we have implemented such methods for solving forward and inverse problems in a fully parallelized fashion for massively parallel computers by Message Passing Interface (MPI). Thus we are able to use more than 10,000 parameters for realistic representation of a 2-D heterogeneous model, far exceeding the resolution (by a factor of 500) that has been accomplished to date by Newton-type seismic waveform inversion.

In previous studies the finite element method was used to solve the acoustic forward problem in the frequency domain. In contrast, we solve the elastic forward problem in the time domain based on the finite difference method and attain greater accuracy by using high order approximation to the derivatives and achieve greater computational efficiency due to the structure of the resulting numerical problem. 


\section{Introduction}

\section{A Theme}

This SEFD project ("Simulation-Enhanced Fracture Detection," Contract \#DE-AC2600NT40689) is a three-phase effort to develop, implement, test and demonstrate a novel strategy for characterizing the state of fractured reservoirs in the subsurface via remote technologies. This report summarizes Phase II accomplishments and concludes with recommendations for Phase III.

In our SEFD approach, computer simulation of the evolution of the subsurface over geological time is used to enhance the inversion of remote data (e.g. seismic reflections) and arrive at more refined physical images (e.g. fracture characteristics and other reservoir properties) than the more typical display of velocity anomalies. In our procedure difficulties in basin modeling are overcome by model/data integration, while the model facilitates the interpretation of remote data. In this way, basin modeling and seismic inversion become one seamless activity, with the strengths of one compensating for the weaknesses of the other. This data/model integration is the essence of our SEFD technology.

This project arose out of a need to integrate a number of approaches for delineating the state of fracturing in the subsurface. Our specific strategy is to develop a computational framework to realize and automate this integration. As the project evolved we deepened our understanding of the challenges to be addressed and thereby arrived at an approach that is both novel in vision and has practical, attainable goals within the scope of the project.

Our vision starts with recognition that the industry, as well as geological science itself, must address the great uncertainty we have in the state of the subsurface. Rather than view this uncertainty as a barrier, we use it as the starting point of our approach. A classic measure of the uncertainty in the state $\psi$ of a system is the entropy $S$. Uncertainty is usually expressed in terms of probability $\rho(\psi)$. Thus $\rho$ is the probability of the state $\psi$, and entropy $S$ is to be expressed in terms of $\rho$. The objective in such a philosophy is to construct $\rho$ by admitting maximum uncertainty $S$ that could be present in light of what is 
actually known - i.e. seismic, well log, production and other data in the present context. In this way, one arrives at an objective assessment of uncertainty. We have developed this general approach, using it to construct the most probable state of the subsurface in light of the available data. This framework yields an automatable algorithm for constructing the subsurface state.

The question then arises as to the details of how we can use the aforementioned data to construct the most probable state of the subsurface. The difficulty is that seismic data is only indirectly related to the spatial distribution of fracturing, porosity, gas versus water or oil saturation, and a myriad of other seismic wave speed-altering factors. Similarly one must address the difficulties in extrapolating information away from the well and interpolating between wells.

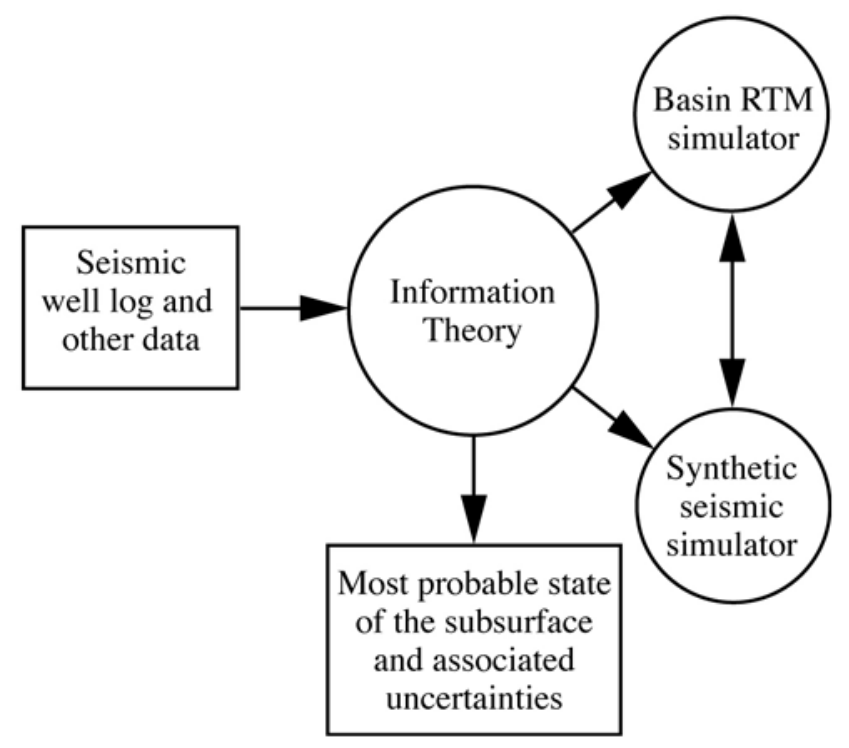

Fig. I.1 Schematic flow for the construction of the most probable state of the subsurface given the seismic and other data. The physics and chemistry of a reaction/transport/mechanical (RTM) basin simulator and a seismic wave simulator is required to translate the RTM model prediction into seismic data. In turn, this is compared with the observed data to arrive at an error measure used in our information theory approach to predict the most probable state of the subsurface.

The overall logic of our approach is depicted schematically in Fig. I.1. The information theory module is the organizing center of the computation. It continuously adjusts the parameters sent to the reaction/transport/mechanical (RTM) basin simulator until the synthetic seismic data created by the seismic simulator agrees with the observed seismic data within acceptable limits. In this sense our procedure is a seismic inversion. However, there are important differences:

- our approach introduces a basin model and thereby constrains the construction of the subsurface state with the laws of physics and chemistry; 
- our approach can be generalized to incorporate other information in a manner that appropriately evaluates each data set with respect to its inaccuracies;

- qualitative information (i.e. expertise) can be folded into the analysis, thereby minimizing losses with personnel turnover;

- as our approach is cast in a probabilistic framework, all predictions can be accompanied with an assessment of uncertainty/risk; and

- many aspects of the procedure can be automated as a computational exploration and production technology.

Taking advantage of these features, implementing them in a reservoir characterization system, and testing/demonstrating this system are the objectives of this SEFD project.

Difficulties of two general types were encountered and overcome in Phase II:

- computational limitations due to the demands of basin RTM simulation; and

- the need for an efficient synthetic seismic program to implement our information theory approach.

Despite these challenges, we have developed a strategy and implemented MAGI (ModelAutomated Geo-Informatics), a software system that we believe to be a major advance in gas exploration and production. Through a major effort, the SEFD project is on track and will be completed in a manner consistent with the original vision in a Phase III effort.

The computational difficulty was overcome via a new strategy that simplified the basin RTM modeling component of our approach. Our strategy is to forego lengthy threedimensional RTM simulations. Rather we calibrated our basin model using a onedimensional simulation at selected sites using well logs and seismic data. The calibrated model was then used to create synthetic one-dimensional data (e.g. mineralogy/texture/pore fluid state/fracture characteristics) at a set of geographic locations and then use this information to stabilize a two- or three-dimensional seismic inversion.

To achieve our goal required the following technical advances:

- two- and three-dimensional seismic wave propagation programs were written that are major advances over pre-existing software due to their ability to simulate wave propagation in a fluid-saturated poroelastic medium with absorbing boundary conditions at the computational boundaries to avoid artifacts to arrive at the most efficient simulator available; 
- an information theory framework that allows for the integration of multiple data types of a range of quality;

- a seismic waveform inversion approach based on the reciprocity theorem, a regularization approach for noise reduction, and the elastic wave equation to use all the information contained in seismic data effectively;

- a MAGI user interface for input manipulation and archiving of user information about a study area;

- a more computationally efficient one-dimensional basin RTM simulator;

- a relational database of geological information that is compatible with the input requirements of our MAGI system and has the requisite stratigraphic, structural, thermal, geochemical, fracturing and other information across the Harrison County, Indiana, study area; and

- seismic data was gathered and preprocessed for input into our software system and chosen specifically to allow us to carry out our test for Phase II.

All the above software and database were written/collated by us constituting an extraordinary accomplishment that in many ways exceeded the original project scope but which was necessary to accomplish our goals.

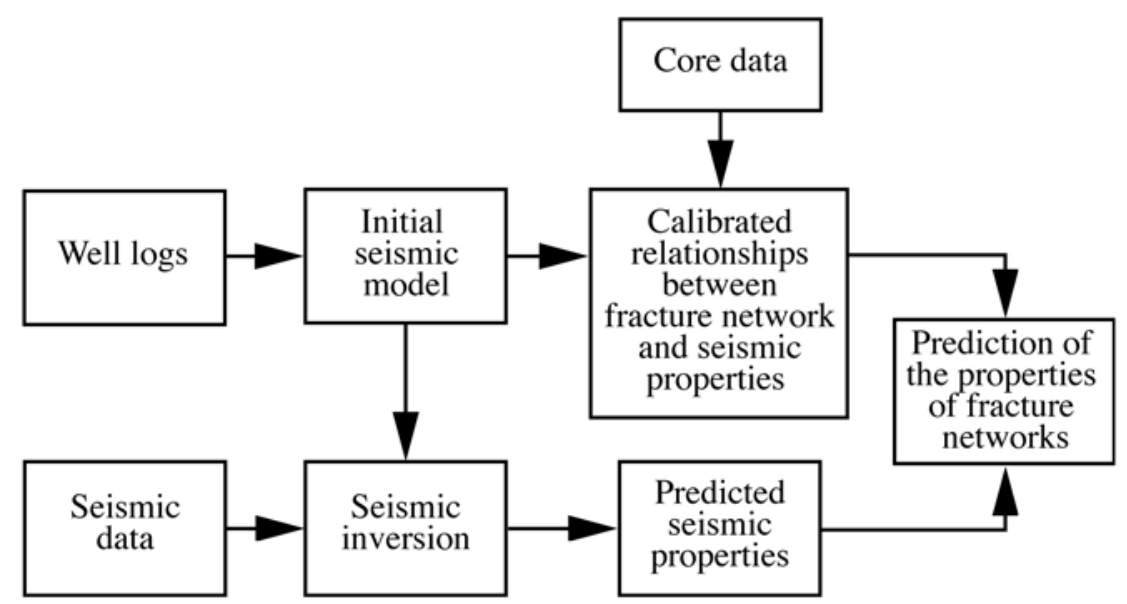

Fig. I.2 Schematic workflow for remote fracture network discovery and characterization.

Our information theory approach also allows for a new inversion process that does not involve basin modeling. The approach is outlined in the workflow of Fig. I.2. Special features of this option, which are embedded in the workflow of Fig. I.1, are as follows. Most geophysical inverse problems have been solved by linearized iterative inversion. The 
forward simulation is approximated by a set of equations linearized about a reference model and a solution of the resulting linearized inverse problem is computed. Then the solution is used as a new reference model for the next step and the process is repeated until convergence. This is a type of Newton method, and a Gauss-Newton approach is typically used. However, for seismic waveform inverse problems, this procedure is too computationally expensive to be practical. Instead, seismic waveform inversion has been accomplished by the gradient method (Lailly 1983; Tarantola 1984) wherein it was shown that the adjoint state method for constructing the gradient direction for the inversion of the acoustic problem could be determined without computing the partial derivatives explicitly.

Recently, as computing resources have been increased, several investigations have been carried out to solve seismic waveform inversion via a Newton-type method. Pratt et al. (1998) implemented the Newton method with "virtual sources" but they parameterized only 4 cubic spline node points of the velocity model; in contrast, we can use 10,000 spline points. Shin et al. (2001) applied the reciprocity theorem to the virtual sources but they did not use a full matrix, keeping only diagonal elements of the approximate Hessian matrix; in contrast, we use all elements of the matrix and therefore obtain a higher quality result. Hicks and Pratt (2001) proposed a two-step inversion procedure which combined the gradient and Newton methods. For the gradient method they used 95,046 parameters, but only 15 parameters were used for the Newton method; in contrast, we can solve the problem with one step of the Gauss-Newton method with more than 10,000 parameters. In all of these studies the finite element method was used to solve the acoustic forward problem in the frequency domain but we solve the elastic forward problem in the time domain based on the finite difference method and attain greater accuracy by using high order approximation to the derivatives and achieve greater computational efficiency due to the structure of the resulting numerical problem.

Newton-type seismic waveform inversion requires huge amounts of memory and computation, far greater than that of a typical scientific workstation. In order to overcome this computer resource limitation, we have implemented such methods for solving forward and inverse problems in a fully parallelized fashion for massively parallel computers by Message Passing Interface (MPI). Thus we are able to use more than 10,000 parameters for realistic representation of a 2-D heterogeneous elastic model, far exceeding the resolution 
by a factor of 500 that has been accomplished to date by Newton-type seismic waveform inversion.

\section{B Meeting the Phase II Plan of Work}

In the following chapters we give an accounting of how we met the objectives of the proposed Plan of Work. All activities focused on Task 4 of the plan of work for the full proposal entitled "Test Fracture Prediction Capability of SEFD in Antrim Shale Fields." Task 4 involves subtasks, to each of which we dedicate a chapter.

\section{Remote Detection of Reservoir Properties Achieved Through the MAGI System}

The technical and conceptual advances of this project have been integrated and implemented into the Model-Automated Geo-Informatics (MAGI) software system. MAGI combines the following elements:

- a basin model to constrain the relationship of the spatial distribution of reservoir characteristics with depth and geological setting;

- $\quad$ seismic wave propagation model and an implementation of the reciprocity theorem to efficiently establish the relation between reservoir characteristics and the seismic signal;

- information theory to construct the most probable state of the subsurface given seismic, well log and other data; and

- graphical techniques to depict the predicted state of fractures and other reservoir characteristics in the subsurface. 


\section{Review, Quality Screen and Organize Data for Test Cases (Subtask 4.1)}

The goals of this subtask were to develop a database of information on a range of geological, reservoir and other information, create a relational database, and structure the data for use with our SEFD software. The data for this subtask was taken from the Illinois Basin (Fig. II.1) with a particular emphasis on the New Albany Shale (Upper Devonian and Lower Mississippian) gas play in Harrison County, Indiana (Fig. II.2). This area will be used to test and demonstrate our SEFD approach.

\section{A Illinois Basin Test Area}

New Albany Shale is an unconventional shale gas reservoir with significant production potential. The total gas content of the New Albany Shale in the Illinois Basin has been estimated to be 86 trillion cubic feet (TCF) by the Devonian Shale Task Group of the National Petroleum Council Committee on Unconventional Gas Sources (Bookout 1980). Although the New Albany Shale has produced commercial quantities of gas for more than 100 years from many fields in southern Indiana and western Kentucky, only a small fraction of its potential has been realized. It is commonly accepted that the reason for the low gas recovery is very low matrix permeability and that natural fractures must be present for the shale to act as an effective gas reservoir.

Fractured gas reservoirs constitute a huge and relatively untapped unconventional resource that is expected to contribute significantly to the national and global gas supply during the next 15 years. A key to successfully exploiting this resource is to develop reliable methods to detect fractures in the subsurface. The fact that New Albany Shale has a long documented history as a fractured gas reservoir in southern Indiana and is currently a target for gas exploration and development makes it an ideal candidate on which to test our simulation enhanced fracture detection methodology.

The area chosen for testing is Harrison County, Indiana, and the immediate vicinity (Fig. II.2). Natural gas seeps were first noted in the bed of the Ohio River in Indiana in 1870 (Collett 1879) and in 1885 drilling for New Albany Shale gas began in Harrison County (Sorgenfrei 1952). To date, most of the gas produced from the New Albany Shale in Indiana 
has been from Harrison County and sufficient subsurface geological data are available to run the simulations and evaluate the validity of our new fracture detection model.

The New Albany Shale consists of interbedded dark-gray and medium-greenish-gray shale, with less abundant beds of argillaceous dolomite in the lower part of the section. Porosity of the New Albany Shale in a core from Christian County, Kentucky, varies from 0.5 to 3.1 percent, averaging 1.8 percent. Porosity of the New Albany Shale in a core from Sullivan County, Indiana, varies from 0.6 to 9.3 percent, averaging 4.0 percent (Kalyoncu et al. 1979). Matrix permeability values in a core of the New Albany Shale from Clark County, Indiana, varied from $2.5 \times 10^{-6}$ to 1.9 millidarcies and had a geometric average of $1.4 \times 10^{-3}$ millidarcies (Zielinski and Moteff 1980). Core analysis of productive zones in the New Albany Shale indicate that fractures provide the effective reservoir porosity that allows recovery of sufficient volumes of gas to make this unit a commercially viable exploration target. 


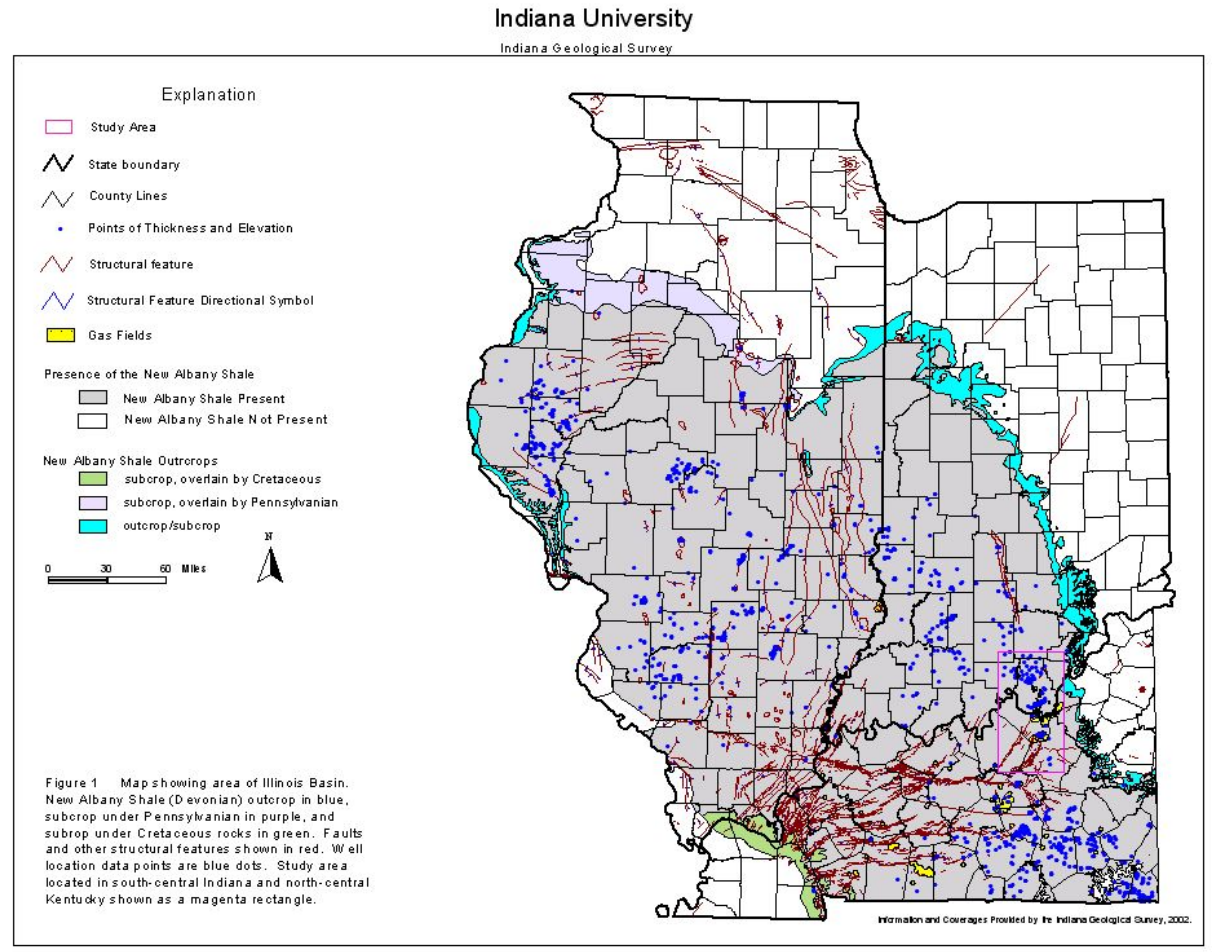

GIS Coverage Showing Structural Features of the Illinois Basin

Fig. II.1

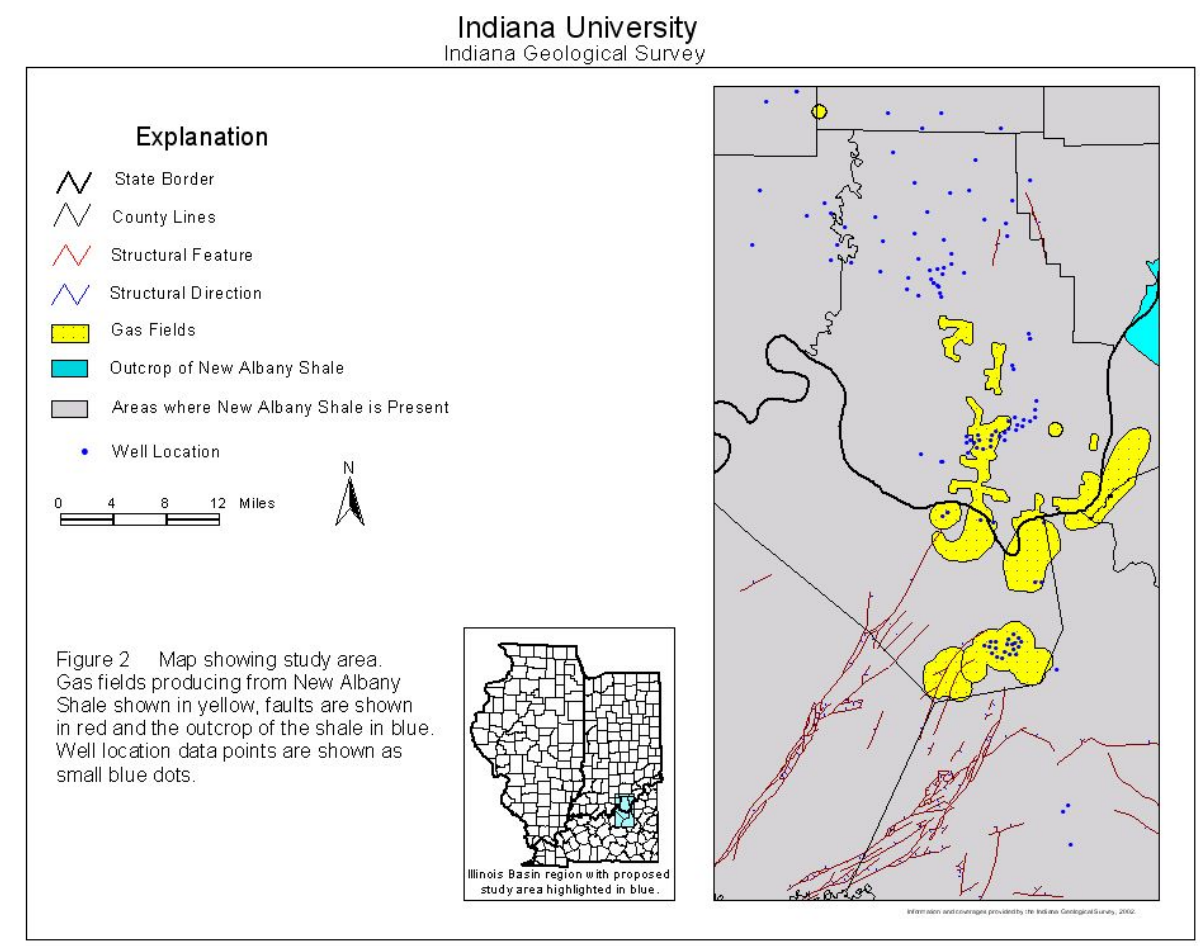

GIS Coverage Showing Structural Features and Gas Fields of the Study Area

Fig. II.2 


\section{B Introduction to the Database}

A database was compiled in Microsoft Access 2002 that contains all of the input and output data that was used for our basin simulations. The database provides optimum organization so that observed and simulated data can be quickly and efficiently compared and analyzed. The Access database allows for simple querying of the data and for exporting data to Geographic Information System (GIS) software. Extracting a table containing selected data is simple and the table may be easily imported into our GIS (Environmental Systems Research Institute, Inc [ESRI] ArcGIS 8.x). The ability to retrieve and analyze data from the large dataset compiled for this project and the ease of use with the ESRI GIS software makes our Access database an ideal platform for storing the data required for testing our automated fracture detection simulator. The database and its instructions may be found on the accompanying $\mathrm{CD}$. An overview for using the database is given in Section $\mathrm{C}$ below.

As GISs have become increasingly popular, their uses have become increasing sophisticated. In addition to making maps and showing locations, innovations allow for 3-D viewing of the surface and the subsurface. Using new versions of the software along with the observed data and data output from the fracture detection simulator, traditional visual representations of the subsurface can be created. Displaying the predicted locations of fractured gas reservoirs in an objectively rendered three-dimensional view should greatly assist exploration and development efforts and reduce risks for petroleum geologists, engineers, and managers. Petroleum companies may expect to increase profits as a result of the reduced risk derived from our objective and probabilistic approach that uses modelautomated informatics to predict the location of fractured reservoirs. As GIS technology advances and prices drop, more companies are likely to invest in this tool for making their

geological and engineering models. The demand for GIS is only likely to increase because it accommodates the need for displaying and analyzing large amounts of data with precise geographic attributes. Using GIS coupled with our automated fracture detection simulator provides a powerful tool for locating fractured gas reservoirs and reducing the financial risks inherent in their exploration and development.

Because porosity data from core analyses is limited to small intervals in producing formations in Indiana, a synthetic porosity log was created for Harrison County, Indiana. This curve includes data from core analyses from regions around Harrison County and also 
data derived from crossplots of well log information. Although the proportion of section represented is small, the porosity data are considered to be of optimum quality. The log data were acquired using a crossplot of neutron porosity and bulk density overlaid with a Schlumberger curve. This approach was used to fill in the stratigraphic gaps from the core analyses. In addition, using both core and log porosity data allows for comparison and evaluation of the quality of the log data. Log crossplot data that matches up reasonably well with the core analysis data is considered to be valid. This practice is commonly used in the petroleum industry and provides critical information on the porosity of the subsurface formations.

Information from the core analyses and the crossplots was then placed into a program (Rockware's LogPlot 2003) to make a synthetic porosity log. These porosity curves can be compared with porosity curves from values output by the simulator, thereby allowing for an in depth comparison and analysis of the simulated and observed data. The LogPlot software allows for multiple curves to be made from different time periods which show the changes in porosity of the rock through time. This software facilitates the data analysis by converting simulator output into the traditional well log format commonly used in the petroleum industry. Agreement between simulated and observed porosity also allows for confidence in interpreting the simulated porosity evolution through time. Because porosity is influenced by fractures, the simulated porosity predictions aid in determining the location and distribution of fractured reservoirs.

\section{Instructions for Using the Database}

The database was designed to store output from the simulator along with observed presentday data gathered from the study area. This is a simple and quick look at how to navigate and use the database to its full potential.

Load the $\mathrm{CD}$ into your computer $\mathrm{CD}$ drive and wait for the $\mathrm{CD}$ drive window to open. Double-click on the file called SimulationOutput.mdb and follow the directions on the screen. 
The first screen that you will come across will look like this:

\section{Form1 : Form}

\section{Please Select an Action From the Following:}

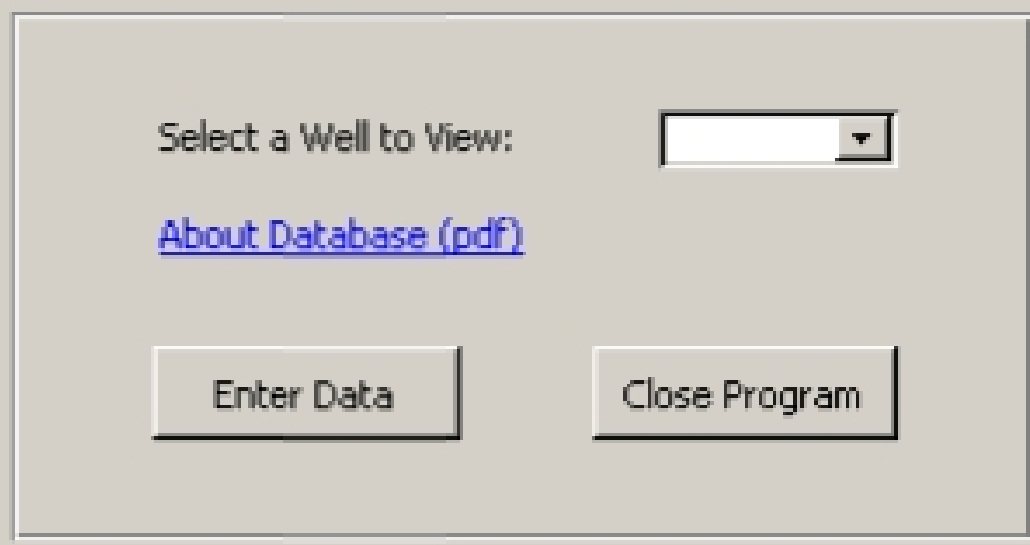

Here you have two options to work with the database:

- Click on the "Enter Data" button, and you will be given a chance to enter new information into the database. Later this information can be viewed by following step 2 .

- Click on the "Select a Well to View" drop down box. The numbers in the drop down list are well identification (ID) numbers. Click on the desired well ID number to view the information in the database for that particular well. 
Clicking on the "Enter Data" button opens the "key" screen that looks like this:

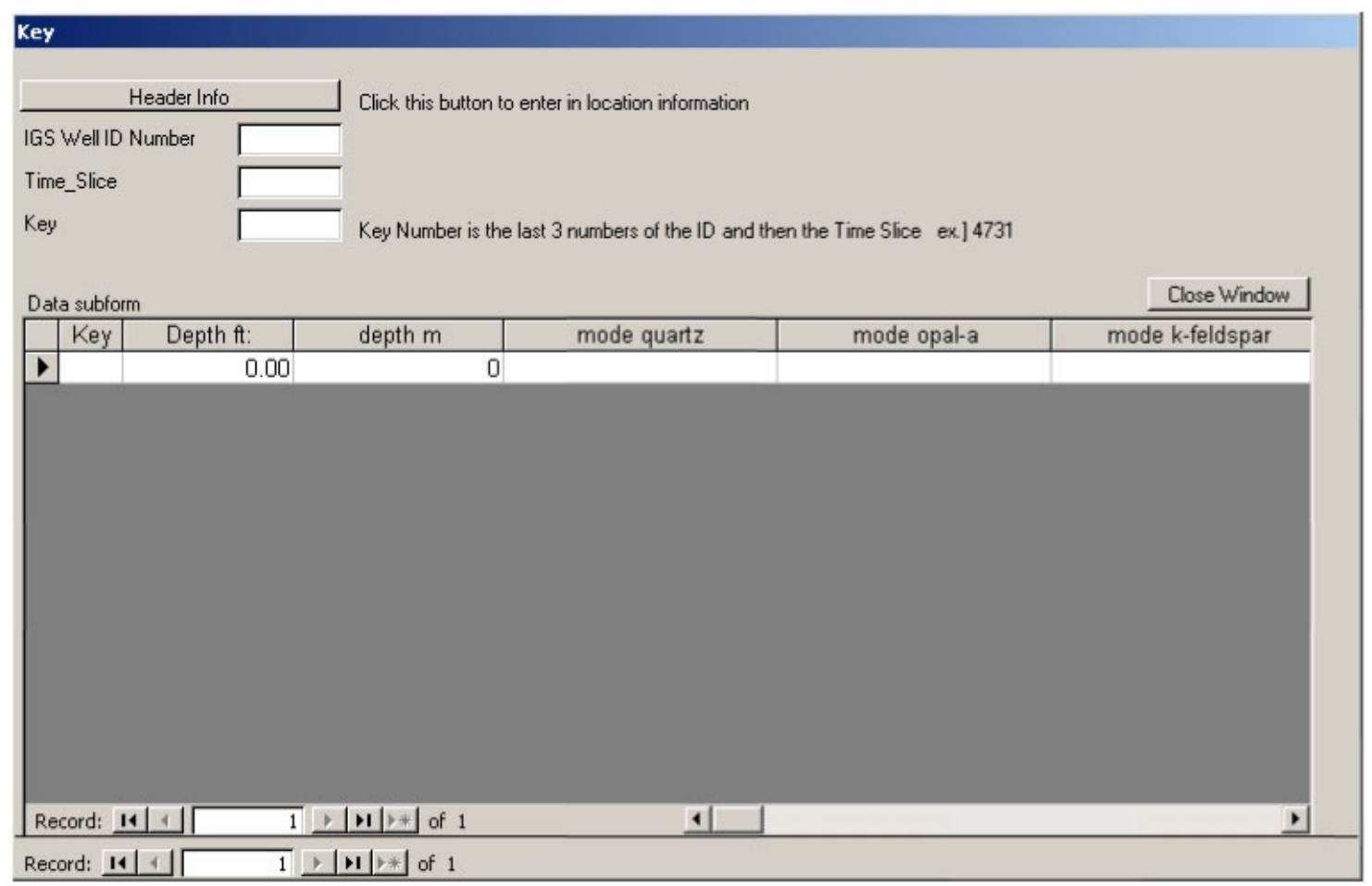

This table allows you to enter data from the simulator by a simple cut-and-paste method. However, you must first click on the "Header Info" button and type in the well location information.

Once the header information has been entered, data from the simulator may be entered. Data output from the simulator is in a text file format that can easily be imported into a spreadsheet. To copy data from the spreadsheet to the database:

- Select the information that you want to copy (do not include column headings).

- Copy the information onto the office clipboard (Edit Copy).

- Select and highlight the first row in the data entry window from the depth $\mathrm{m}$ heading to the last column on the right.

- Paste the information into the database window (Edit Paste).

Once you have entered the data for one time slice, press the record advance button in the bottom left corner to advance the window to the next time slice. When you have entered in all the information that you want, close the window by clicking the "Close Window" button and go back to the main screen. 
The header table for entering location information looks like this:

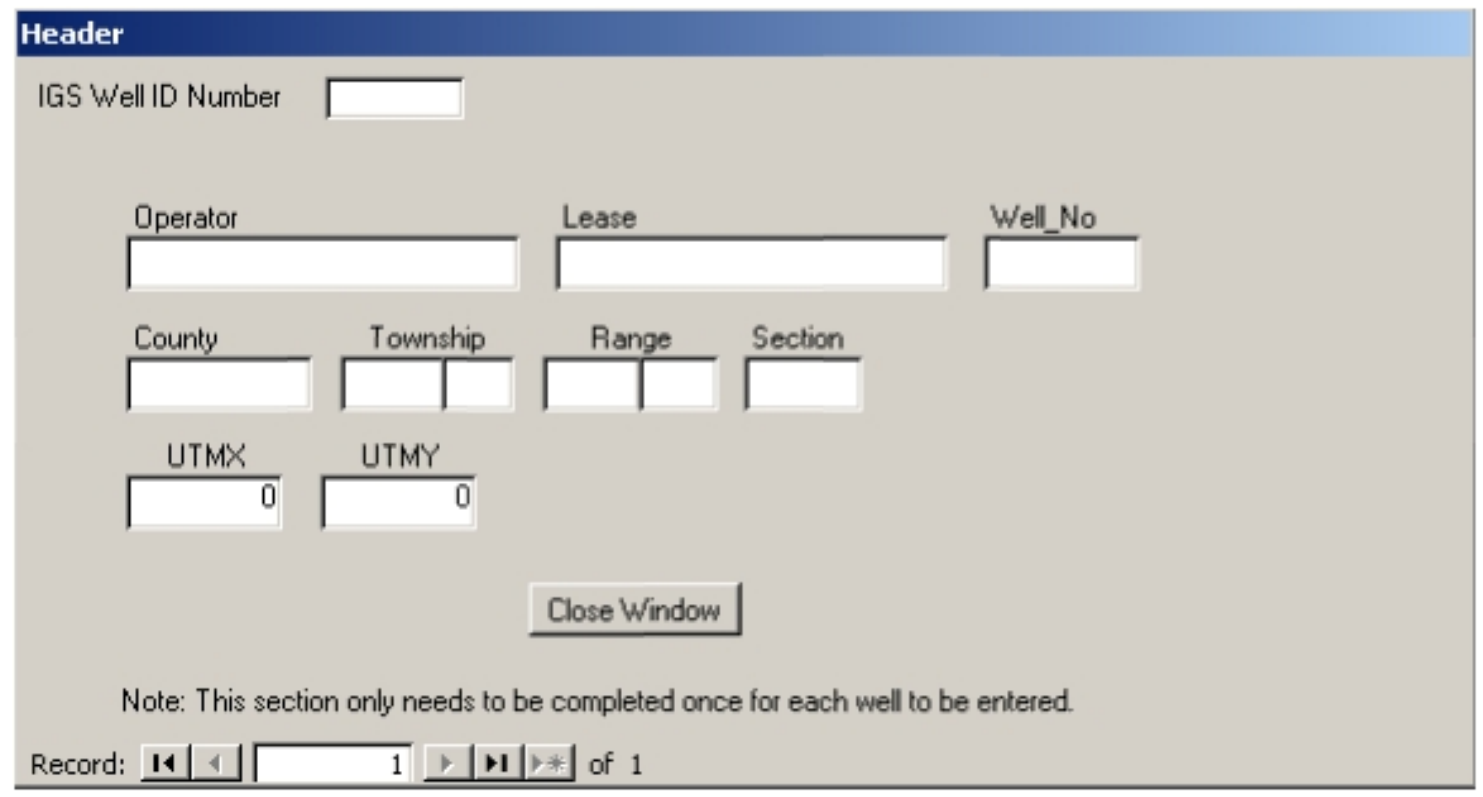

This table allows for the location information to be entered so that a well may be uniquely identified. Enter the operator's name, lease name, etc. as requested. As noted at the bottom, this section only needs to be completed once per well. Be sure to enter the UTMX and UTMY values, otherwise the data cannot be used in a GIS application. After entering in the location information, close the window and go back to the data entry window.

\section{Form1 : Form}

\section{Please Select an Action From the Following:}

Select a Well to View:

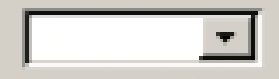

About Database (pdf)

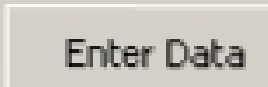

$$
\text { Close Program }
$$


Back at the main screen, choose a well from the dropdown list in order to view what information is available for that particular well. The wells are listed by well ID numbers (which are in numerical order). Click the down arrow and click on the well number that contains the information that you want to view. Click "here" to view the resulting window after choosing a well to view.

When a well ID is chosen (well 124473 has been chosen as an example) the screen below will open:

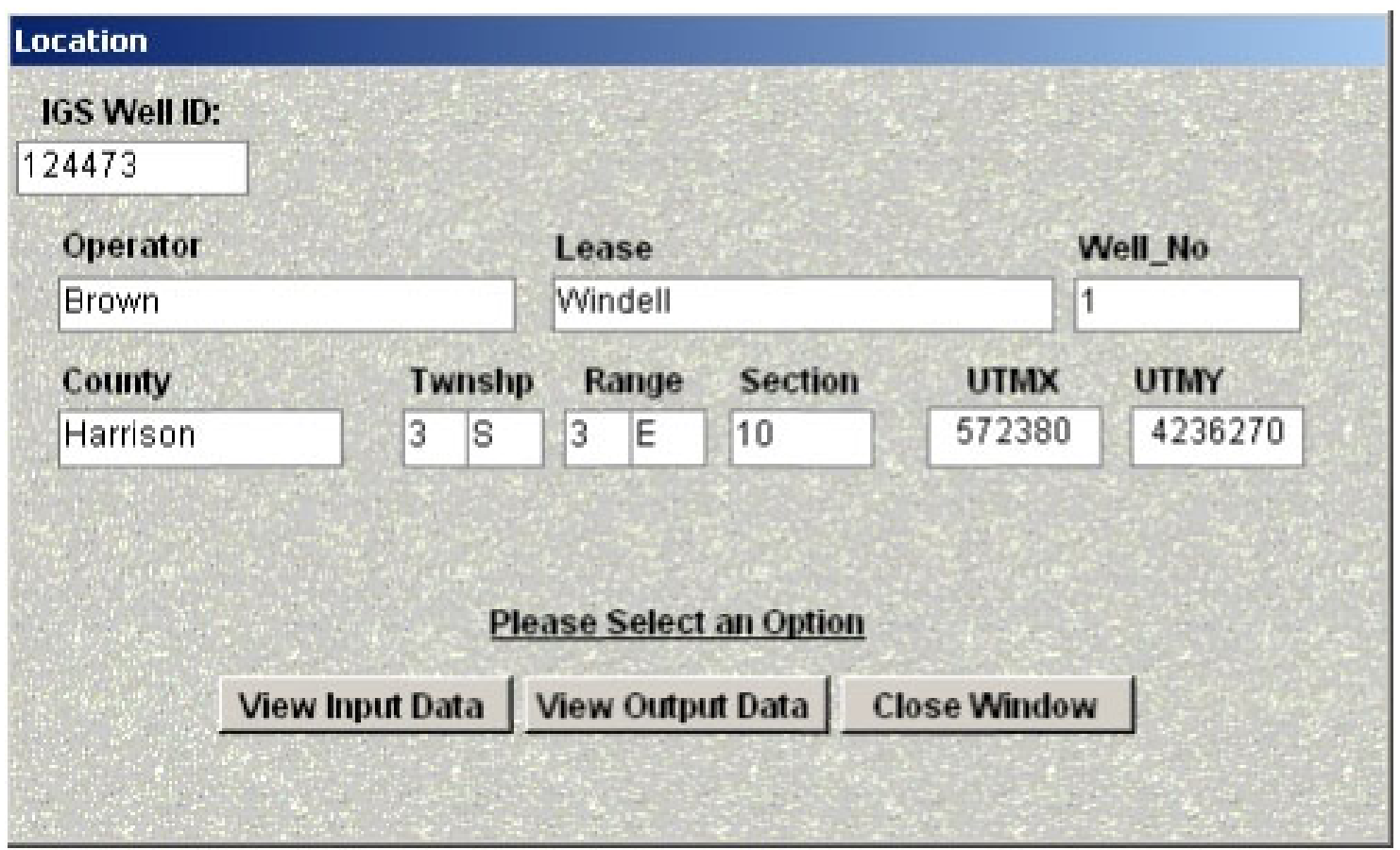

This screen shows the location information including the ID number, the operator, lease name, well number, county, township, range, section, and GIS map coordinates (UTMX and UTMY). This information allows the user to locate the well on a paper map using township, range, and section and to plot the location using GIS application. The UTM coordinates are in Universal Transverse Mercator coordinates, 1983 North American Datum, Zone 16.

To view the data either:

1. Click "View Input Data" to view the present-day data for this well, or 2. Click "View Output Data" to view the data that was output from the simulator. 
When the "View Input Data" button is clicked, the screen shown will appear:

\section{Input Data : Form}

IGS Well ID Number: 124473
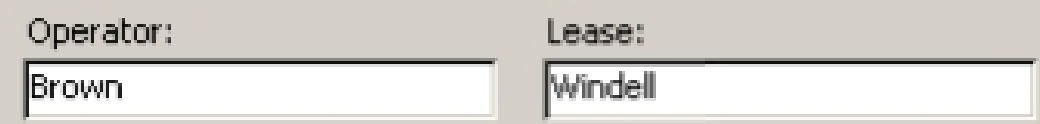

Well Number:

1

Please Select a Table From the Following:

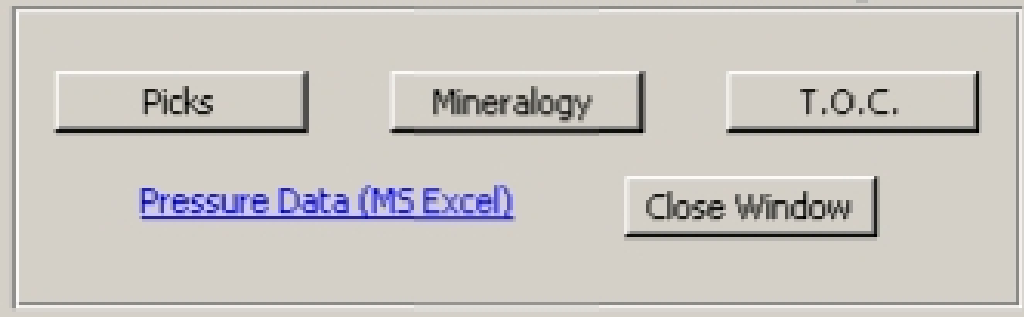

This screen shows the ID number along with the operator, lease and well number so that you are sure you are looking at the correct well. There are three tables that you may choose to view. Each of these tables contains essential data that was input into the simulator to calibrate the program. The names of the tables and a brief description are given below:

1. Picks: This table shows the present day depths to the top of the geologic group/ formation. These "tops" were determined using geophysical logs and maps depicting the average thickness and depth to the top of the formations. Not all of the tops were represented on the well logs so some inferences were made using the maps and were thus denoted as "projected" in the table with a check in the appropriate check box.

2. Mineralogy: This table shows the proto-mineralogy or what the composition is thought to have been at the time of deposition. A list of the most common minerals is given and its associated volume fraction along with the approximate grain size given in centimeters.

3. TOC: The total organic content present in the rock. This is given as a volume fraction that represents the amount of organic material though to be present in the rocks at the time of deposition. 
4. Pressure Data: This is a spreadsheet file that opens in Microsoft Excel and contains pressure data from the Devonian group. The area covered is southwest Indiana and contains standard pressure data entries and terminology.

When viewing these tables, use the record advance buttons in the bottom left corner of the window. To close each window, click on the "Close Window" button in each window. Click "here" to go back to the location table.

When the "View Output Data" button is clicked, the screen shown below will appear:

\section{Form2 : Form} Well ID Number:

124473

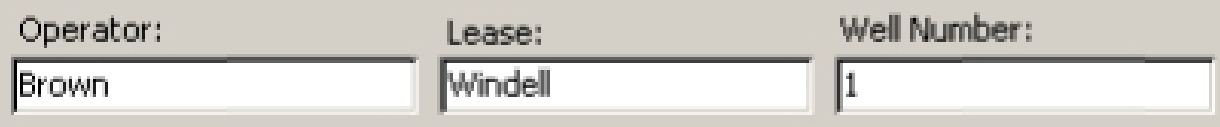

Select an Action From the Following:

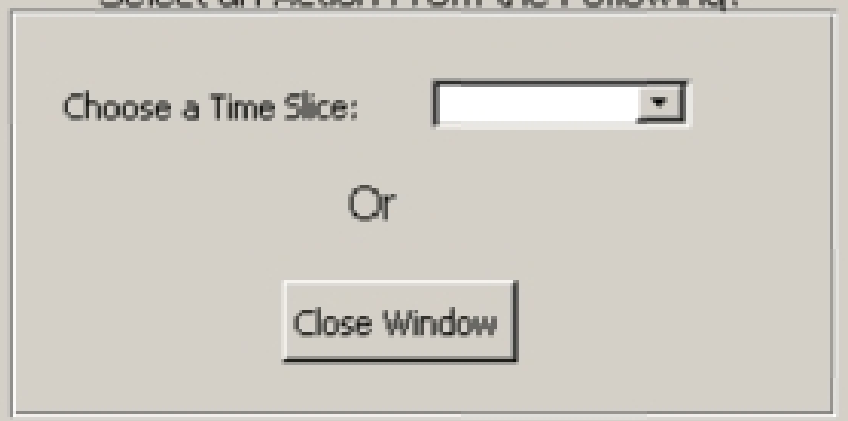

Click the dropdown button to view a time slice. This will limit the amount of data to view and it will allow you to more easily compare observed data to the simulator output. A time slice includes all the data that represents the condition of the basin at a given instant in geologic time. There are 130 time slices per well with each time slice representing a different set of data. Time slice 1 is the oldest and has the least amount of data, while time slice 130 represents simulated present day conditions. Choose a time slice to view by clicking the dropdown box and selecting the time slice you want to view. You are then taken to a corresponding data table. For an example, click "here". 
When the time slice is selected (well number 124473, time slice 1 is being used as an example), the following screen will appear:

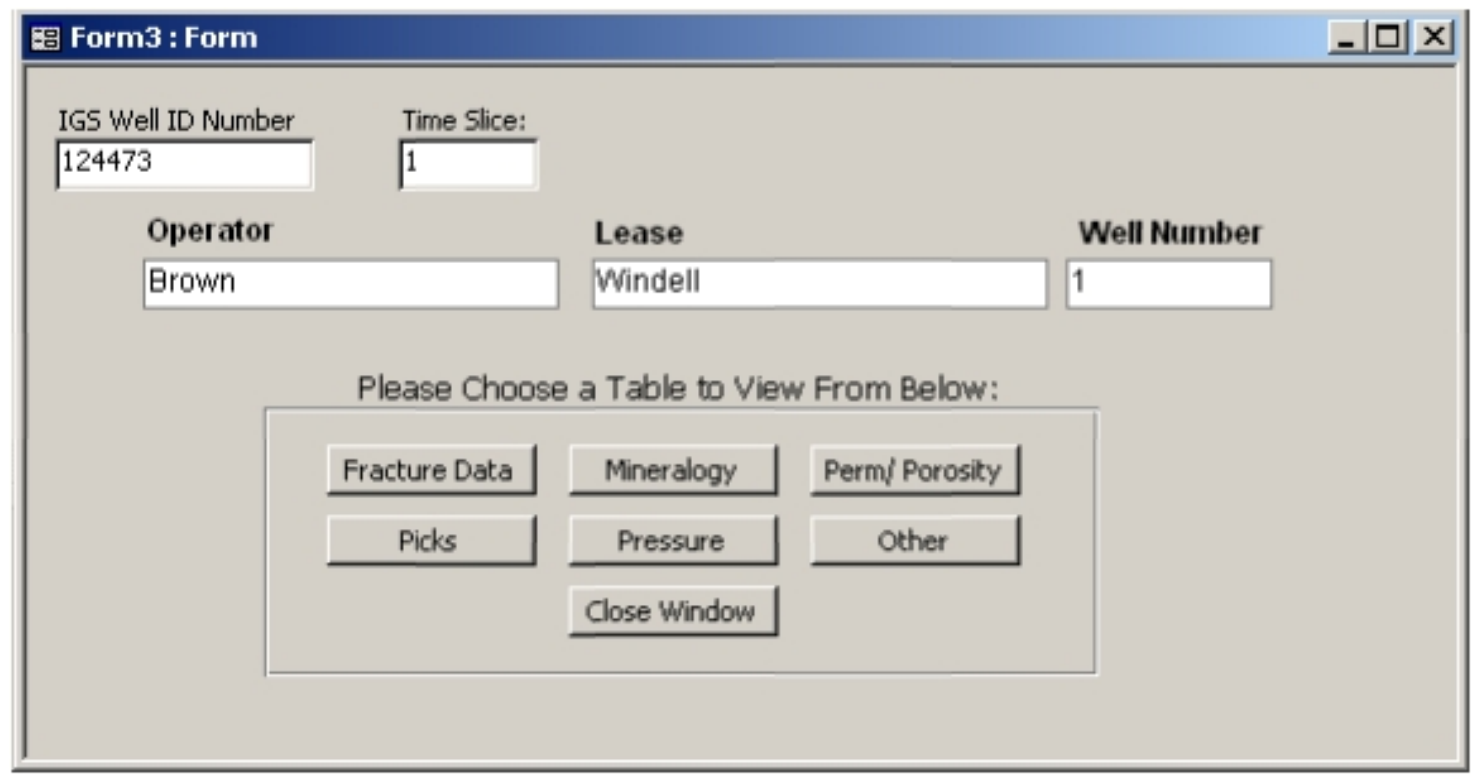

This screen looks similar to the input data screen but shows the time slice along with six tables. The data output from the simulation was grouped into categories based upon characteristics and data type. Below is a brief description of each of the tables and their contents:

1. Fracture Data: This table contains all the data on the fractures predicted by the simulator. The data for each time slice is arranged by depth, which means that the data is ordered by geologic formation.

2. Mineralogy: This table represents the mineralogy of the geologic formation. A list of the most common minerals is given along with their approximate volume fraction. Also given is the grain size expressed in centimeters.

3. Perm/ Porosity: This table contains all the information related to permeability and porosity that is output by the simulator. The data for each time slice is arranged by depth, which means that the data is ordered by geologic formation.

4. Picks: This table shows the depths to the geologic formations. Many formations have multiple depth entries which represent different time lines through the same geological formation. Only the last depth listed for a given formation represents the top of that unit. Also the present day depth is calculated here for convenient comparison with the input 
data. Use this table when comparing the tops of the observed formation to the tops of the output by the simulator.

5. Pressure: This table contains all of the pressure information that is output by the simulator. The data for each time slice is arranged by depth, which means that the data is ordered by geologic formation.

6. Other: This table contains all of the miscellaneous information that does not fit into one of the above tables. The data for each time slice is arranged by depth, which means that the data is ordered by geologic formation.

This concludes the overview of the SEFD database. These instructions are also included on the CD in the SEFDAbout.pdf file. 


\section{Implement the Web-Based User Interface (Subtask 4.2)}

\section{A Overview}

The goal was to realize the practical goals of SEFD by developing a user-friendly end-toend system of data entry, seismic physical imaging, and graphical tools for result interpretation. The web interface called MAGI (Model-Automated Geo-Informatics) was constructed to simplify the process of collecting geologic data and performing simulations using information theory (IT). One can use this interface from any computer to enter well and lithology data into a database, edit it, and select wells to include in a simulation; to specify various parameters and settings for a simulation and create the appropriate input files; and finally, to submit a job to a specified machine and monitor its progress. The interface is programmed in PHP, using mySQL for the database component. Javascript has been used occasionally for pop-up windows. The look has been optimized for the web browser Mozilla.

\section{B Users, Directories, and Databases}

MAGI includes a Users table in the administrative database, which records each user's information, such as name, affiliation, mail and email address. Each user belongs to a group of one or more users, and each member of a group has access to the other members' files and data. To ensure privacy, members of one group never see the files or data of other groups. (Administrators can facilitate sharing of information by putting files into the admin/examples/ directory, which is available to all users.)

To use MAGI, one has to login using a password. Only authenticated users have access to the web pages. Each group can have a number of databases containing well and lithology data. It is expected that each "project" the group is working on would correspond to a different database. Databases are named geo_[group_name]_[proj_name]. Currently, any user can create a new database for his group and any user can edit the data in his group's databases. See the next section for more details about the databases.

Each group is also given directory space ([group_name]/) on the web server and each user is given a subdirectory in that home directory ([group_name]/[user_name]/). It is 
expected that each user will normally keep all his files in his own directory, to avoid confusion. To do simulations, the user must create a subdirectory corresponding to the database containing the data ([group_name]/[user_name]/cirf/[proj_name]/). Input and output files for/from the runs are located in this directory and its subdirectories.

A "Manage Files" web page allows the user to view his directories and files; delete, copy and move files; create and delete subdirectories; and upload files from a home computer. Another web page automates creation of a new database and/or project directory (with subdirectories).

Each geologic database consists of 5 tables: wells, layers, lithologies, lith_compositions, and minerals. These database tables contain all the choices that the user will have when he or she sets up the input files for a simulation. For example, all the known wells in an area can be recorded in the wells table, but not all have to be used in a particular calculation. Fig. III.1 shows the contents of the tables and how they are connected.

Basically, the wells table contains information relevant to each well as a whole (e.g. the well name and ID and its location). The layers table contains the tops data for each well-the age, depth, and lithology of each layer in the well. The lithologies table consists of a list of lithologies that may be found in wells and their overall characteristics. The composition of each lithology — the minerals and organics that it is made of - are found in the lith_compositions table. Currently, the minerals table is a list of minerals that may be used in the lith_compositions table. Eventually, physical properties of each mineral could be included.

Users have the option of reading well data from a file with a specific format (a comma-separated text file), reading from a previously written "Project File" (see below), or adding wells by hand one at a time. As users request, special scripts can be written to allow data to be imported from files in other formats. Lithology data can be entered by hand or read from a previously constructed Project File. The user will be able to read example Project Files to quickly obtain basic lithology data. Mineral data is currently put into each database automatically when the database is created. An administrator would have to edit the list for a user if changes were desired.

Any of the data in the database can be displayed and edited via convenient forms produced by MAGI (except that the minerals table cannot currently be edited). Web pages 
with tables of wells and lithologies show basic data for each and allow the user to select one for editing or deletion. After selecting a well or lithology, layer and composition data can then be displayed, edited, added to, or deleted. When a change is made, the date and username of the person who did the editing are recorded in the database, as well as an optional comment. Old versions are not actually deleted but will not normally be displayed.

\section{Setting up for a Run}

Once the user has set up a database and a directory and filled the database with data, he or she is ready to prepare for doing a simulation. Each IT run requires several inputs, the main ones being the Project File and the Session File. The Project File contains all the data and settings necessary to do a single cirfb run. (Each IT, or Tropy, run involves many cirfb runs.) It contains all the geologic data, the related lithology data, material properties, and control settings. The Tropy run is controlled by the Session File. This file contains information such as which machine to run cirfb on, where the files are that contain observed data, and the location of the files required by the synthetic seismic routine.

\section{The Project File}

The Project File contains all the well, layer, lithology, and lithology composition data to be used in the simulation. In MAGI, the user first specifies the database from which to obtain the data. He can also choose another previously written Project File from which to select wells. From the list of wells in the database and the specified Project File, the user then simply selects the wells to include in the simulation. All the related data (layers and lithologies) are gathered and written to the Project File automatically.

After selecting the wells, the user selects the thermal profiles (temperature versus time, at a specific location) to use in the simulation. Default profiles are presented, which the user can edit and save for future use in a file. The profiles that are selected are written to the Project File.

The user than chooses the control settings, material properties, and output variables. Currently, the user is not able to edit these individually, but a preset combination can be chosen from the files in the MAGI example directory (or elsewhere in the group directory space). The contents of the three chosen files are copied into the Project File. Finally, the 
user specifies a directory and root filename for cirfb output and tells MAGI what to name the Project File (PROJDAT.something) and where to write it.

\section{Other Input Files}

Before doing an IT run, one must also have a synthetic seismic input file in the project directory. The user is presented with a list of variables with default values. These may be changed or used as-is. The default values are kept in an administrative database table, so they are easily updated, though not edited by the user. The changed values are written out automatically to the project directory in a file that the user names.

An IT run also requires another input file for the synthetic seismic routine: sweep3.dat, as well as files containing observed data of various types. Currently, these cannot be modified by the user, but default versions created by administrators can be found in the MAGI example directory. When a user creates a new project directory, they are automatically copied to it.

\section{The Session File}

The control file for the IT part of a run is the Session File. Again, the user first specifies the project (the database and associated directory) for the run. This determines where the output will go and where the other input files will be found. A form is then presented in which the user can specify the name of the Session (used in the name of the Session File and in the names of the output files), choose whether to do a Newton-Raphson calibration or a grid search, and select which parameters to calibrate via with information theory. The Project File to be used for the cirfb runs must be specified, along with the synthetic seismic input files and the observed data files. These are chosen from drop-down menus.

Finally, the user chooses which version of cirfb to use, where to run the cirfb processes (on the web server or the SP supercomputer), and how many concurrent processes (cirfbs) to run. (Tropy itself always runs on the web server.) MAGI then writes the Session File and the user is ready to submit a run.

\section{Submitting and Monitoring a Run}

To submit a Tropy run (i.e. an IT run) the user clicks on the appropriate button on the main menu, chooses the Session File for the run, specifies whether to start a new run or resume one that was not completed earlier, and clicks the "Submit Job" button. MAGI starts Tropy 
running in the background, and Tropy, following the instructions in the Session File, starts cirfb processes on either the web server or the supercomputer (SP). Tropy keeps track of the cirfb processes and continuously updates various log files and output files, such as the Status File, so that the run can be resumed if Tropy and/or the cirfb processes crash or are killed.

Anytime the user wants to see the progress of a Tropy run, the "Check on an IT Run" button on the main menu can be used to get to the monitoring web page. The user simply selects the Session File of the job to be checked. MAGI looks on the web server for that Tropy job, as well as any of its cirfb processes that are running on the web server. It informs the user of which jobs are running, then reads the Status File of the job for further information from Tropy. If cirfb jobs are running on a remote machine, the Status File will indicate which jobs are running. The web page will automatically reload itself to continue monitoring until the user moves to a different web page. When or if the job has ended, MAGI will show the basic final results as written to the Status File.

\section{E Future Development}

There are many modifications, enhancements, and additions that need to be made to the current version (Version 1). First, the underlying programming and logic needs to be streamlined and standardized so that it is easier to fix bugs and add new features. Using more functions, standardizing naming schemes and ways of doing things, and developing a well thought-out style sheet would be part of this. Without a lot of experience and planning, the user can find PHP and HTML complex and confusing. Although we have attempted to keep things as organized as possible, Version 1 has been a learning experience, and a rewrite for Version 2 would be extremely useful in the long run. The database structure (the particular variables that are stored) should be reconsidered in light of the needs of the users, especially after some testing of Version 1. If the users need to have access to old (unedited) data, to see what combination of values they used at some earlier time, for example, the layer data needs to be more tightly related to the well header data, the composition data to the lithology header data and vice versa. The lithology data also needs to be related to a specific version of a well or layer. If it is determined that old data is not really needed, the database and the program logic can be simplified. 
Other improvements that can be made include

- $\quad$ adding options to look at, plot, and analyze the output.

- $\quad$ adding options to share information and files between users in different groups.

- enabling different classes of users with different permissions with regard to creating and editing database data, and so forth.

- adding material properties (e.g. for minerals), thermal profiles, control settings, and so forth to the database instead of using files, and making it possible for the user to easily edit them.

- adding the ability to use more information from a template Project File and/or Session File, so that a user can copy from what he or someone else has done previously, rather than start from scratch every time.

- allowing the user to edit a Project File without changing the database.

- considering security issues more thoroughly.

- adding the ability to download files from the web server to the user's computer.

- adding more error checking.

- adding a "help" button to each page.

- adding tables to the database where each user's runs are recorded.

- adding options to sort wells and other items in different ways and show only selected variables in the web page tables. 


\section{Develop Efficient Seismic Simulator (Subtask 4.3)}

\section{A Overview}

Seismic methods have been successful in interpreting geologic structures and stratigraphic features, although they generally treat the medium as a single phase elastic solid rather than a fluid saturated porous medium. Pore fluids strongly influence the seismic properties of rocks. However, properties of pore fluids such as density, bulk modulus, saturation and viscosity have been ignored in most studies. In recent years, numerical simulation of wave propagation in fluid saturated poroelastic media has received more attention as its importance in geophysical exploration and reservoir characterization is now recognized (Arntsen and Carcione 2001; Pride et al. 2002).

Numerical simulation of wave propagation in fluid saturated poroelastic media is based on Biot's theory (Biot 1962). The finite-difference (FD) method for Biot's equations has been formulated in several ways; central difference FD method in displacement (Zhu and McMechan 1991; Zeng et al. 2001), velocity-stress predictor-corrector FD method (Dai et al. 1995), and velocity-strain staggered-grid FD method (Zeng and Liu 2001). Because central difference operators to perform first derivatives are less accurate than staggered-grid operators for high frequencies close to the Nyquist limit (Kneib and Kerner 1993), we employ a staggered-grid method to increase the accuracy of the numerical discretization.

In order to simulate an unbounded medium, an absorbing boundary condition $(\mathrm{ABC})$ is often used to truncate the computational domain. A commonly used $\mathrm{ABC}$ in seismic modeling is the one-way wave equation based on the paraxial approximations of the acoustic or elastic wave equations (Clayton and Engquist 1977). Recently, the perfectly matched layer (PML) method for electromagnetic problems has been proposed by Berenger (1994) and it has been successfully applied to various wave propagation problems (Chew and Weedon 1994; Zeng and Liu 2001).

In this section, we present a numerical method to solve Biot's equations in heterogeneous, fluid saturated poroelastic media based on a first order hyperbolic formulation whose unknowns consists of solid phase velocity, velocity of fluid phase relative to that of solid phase, solid stress, and fluid pressure. The method of complex 
coordinates (Chew and Weedon 1994) is used to formulate the PML method for the velocity-stress staggered-grid formulation. Furthermore, to increase the accuracy, a harmonic average of material properties is employed (Graves 1996; Moczo et al. 2002).

\section{B Theory}

Biot's theory (1962) takes account of energy dissipation due to the relative motion between viscous pore fluid and the solid matrix. The theory is developed under the following assumptions: (1) seismic wavelengths are larger than the representative elementary volume; (2) deformations in both solid and pore fluid are small in order to remain in the linear regime; (3) the solid matrix is elastic and locally homogeneous; (4) fluid phase is continuous and disconnected pores are treated as part of the solid matrix and the porous medium is fully saturated; (5) seismic response is computed at frequencies low enough so that fluid flow can be described by Darcy's law; and (6) gravity forces and scattering effects due to individual pores are neglected. Biot's equations for a fluid-saturated, statistically isotropic, locally homogeneous, poroelastic medium are given by

$$
\begin{aligned}
& \rho \ddot{\mathbf{u}}+\rho_{f} \ddot{\mathbf{w}}-\left(\lambda_{c}+\mu\right) \nabla \nabla \cdot \mathbf{u}-\mu \nabla^{2} \mathbf{u}-\alpha M \nabla \nabla \cdot \mathbf{w}=0, \\
& \rho_{f} \ddot{\mathbf{u}}+m \ddot{\mathbf{w}}+b \dot{\mathbf{w}}-\alpha M \nabla \nabla \cdot \mathbf{u}-M \nabla \nabla \cdot \mathbf{w}=0,
\end{aligned}
$$

where

u: displacement vector for the solid;

w: displacement vector of the fluid relative to that for the solid;

$\rho$ : the overall density of the saturated medium determined by $. \rho_{f}+(1-\quad) \rho_{s}$;

$\rho_{f}$ : density of the fluid;

$\rho_{s}$ : density of the solid;

$\phi$ : porosity;

$\lambda_{c}$ : Lamé constant of the saturated matrix;

$\mu$ : shear modulus of the dry porous matrix;

$m$ : effective fluid density;

$\eta$ : viscosity of the fluid;

$\kappa$ : permeability of the porous medium;

$b$ : mobility of the fluid defined by $\eta / \kappa$;

$K_{s}$ : bulk modulus of the solid; 
$K_{f}$ : bulk modulus of the fluid;

$K_{b}$ : bulk modulus of the dry porous matrix;

$\alpha=1-K_{b} / K_{s} ;$

$M=\left[\phi / K_{f}+(\alpha-\phi) / K_{s}\right]^{-1}$.

From the definition of strain energy function in porous media (Biot 1962), the stress $\tau$ and the pore fluid pressure $p$ are given by

$$
\begin{aligned}
& \tau_{i j}=2 \mu e_{i j}+\delta_{i j}\left(\lambda_{c} e_{k k}+\alpha M w_{k, k}\right), \\
& p=-\alpha M e_{k k}-M w_{k, k},
\end{aligned}
$$

where $e$ denotes the strain tensor. The time derivatives of the displacements can be written in terms of the stress and the pore fluid pressure:

$$
\begin{aligned}
& \left(m \rho-\rho_{f}^{2}\right) \ddot{u}_{i}=m \tau_{i j, j}+\rho_{f} b \dot{w}_{i}+\rho_{f} p_{, i}, \\
& \left(m \rho-\rho_{f}^{2}\right) \ddot{w}_{i}=-\rho_{f} \tau_{i j, j}-\rho b \dot{w}_{i}-\rho p_{, i} .
\end{aligned}
$$

These equations can be written as a set of first order differential equations in time by differentiating eq. (IV.3) and (IV.4) with respect to time:

$$
\begin{aligned}
& \left(m \rho-\rho_{f}^{2}\right) \dot{v}_{i}=m \tau_{i j, j}+\rho_{f} b V_{i}+\rho_{f} p_{, i}, \\
& \left(m \rho-\rho_{f}^{2}\right) \dot{V}_{i}=-\rho_{f} \tau_{i j, j}-\rho b V_{i}-\rho p_{, i}, \\
& \dot{\tau}_{i j}=\mu\left(v_{i, j}+v_{j, i}\right)+\delta_{i j}\left(\lambda_{c} v_{k, k}+\alpha M V_{k, k}\right), \\
& \dot{p}=-\alpha M v_{k, k}-M V_{k, k},
\end{aligned}
$$

where $v_{i}=\dot{u}_{i}$ and $V_{i}=\dot{w}_{i}$. Eqs. (IV.7)-(IV.10) form a set of first order hyperbolic equations in time for $v, V, \tau$, and $p$.

\section{Finite-Difference formulation}

Eqs. (IV.7)-(IV.10) can be discretized using a staggered-grid FD method (Graves 1996).

The most outstanding feature of this method is that the differential operators are all naturally centered at the same point in space and time (Fig. IV.1). The discretization yields

$$
\begin{aligned}
& v_{x}^{n+1 / 2 / 2, j, k}=v_{x}^{n-1 / 2}{ }_{x}^{i+1 / 2, j, k}+\left.d t\left[A_{x}\left(\Delta_{x} \tau_{x x}+\Delta_{y} \tau_{x y}+\Delta_{z} \tau_{x z}\right)+B_{x} \bar{V}_{x}+C_{x} \Delta_{x} p\right]\right|_{i+1 / 2, j, k} ^{n}, \\
& v_{y}^{n+1 / 2+1 / 2, k}=v_{y i, j+1 / 2, k}^{n-1 / 2}+d t\left[A_{y}\left(\Delta_{x} \tau_{x y}+\Delta_{y} \tau_{y y}+\Delta_{z} \tau_{y z}\right)+B_{y} \bar{V}_{y}+C_{y} \Delta_{y} p\right]_{i, j+1 / 2, k}^{n},
\end{aligned}
$$




$$
\begin{aligned}
& v_{z}^{n+1, j, k+1 / 2}=v_{z}^{n i, j, k+1 / 2}+d t\left[A_{z}\left(\Delta_{x} \tau_{x z}+\Delta_{y} \tau_{y z}+\Delta_{z} \tau_{z z}\right)+B_{z} \bar{V}_{z}+C_{z} \Delta_{z} p\right]_{i, j, k+1 / 2}^{n} \\
& V_{x}^{n+1 / 2}=D_{x} V_{x}^{n-1 / 2,1 / 2, j, k, k}-\left.d t\left[E_{x}\left(\Delta_{x} \tau_{x x}+\Delta_{y} \tau_{x y}+\Delta_{z} \tau_{x z}\right)+F_{x} \Delta_{x} p\right]\right|_{i+1 / 2, j, k} ^{n}, \\
& V_{y}^{n+1 / 2+1 / 2, k}=D_{y} V_{y}^{n-1 / j+1 / 2, k}-d t\left[E_{y}\left(\Delta_{y} \tau_{x y}+\Delta_{y} \tau_{y y}+\Delta_{z} \tau_{y z}\right)+F_{y} \Delta_{y} p\right]_{i, j+1 / 2, k}^{n}, \\
& V_{z}^{n+1, j, k+1 / 2}=D_{z} V_{z}^{n-1, j, k+1 / 2}-\left.d t\left[E_{z}\left(\Delta_{z} \tau_{x z}+\Delta_{z} \tau_{y z}+\Delta_{z} \tau_{z z}\right)+F_{z} \Delta_{z} p\right]\right|_{i, j, k+1 / 2} ^{n},
\end{aligned}
$$

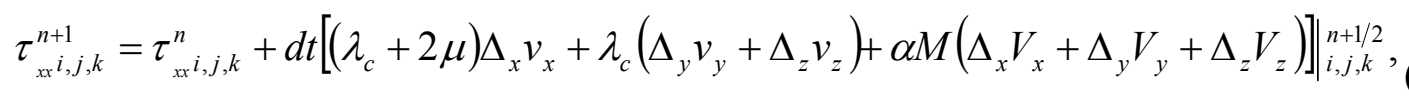

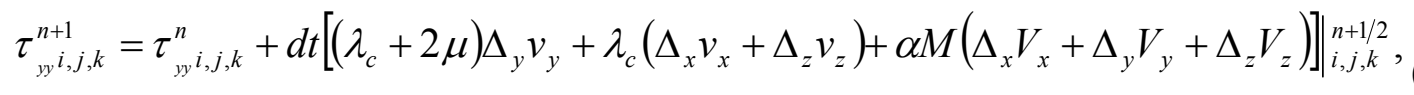

$$
\begin{aligned}
& \left.\tau_{z z}^{n+1, j, k}=\tau_{z z}^{n}{ }_{z i, j, k}+d t\left[\left(\lambda_{c}+2 \mu\right) \Delta_{z} v_{z}+\lambda_{c}\left(\Delta_{x} v_{x}+\Delta_{y} v_{y}\right)+\alpha M\left(\Delta_{x} V_{x}+\Delta_{y} V_{y}+\Delta_{z} V_{z}\right)\right]\right]_{i, j, k}^{n+1 / 2},
\end{aligned}
$$

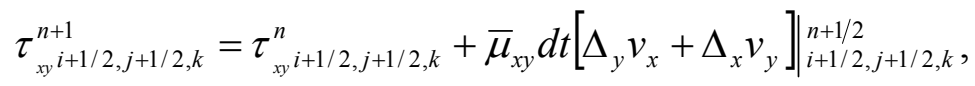

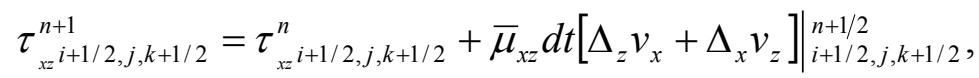

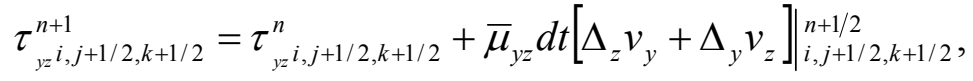

and

$$
\left.p_{i, j, k}^{n+1}=p_{i, j, k}^{n}-d t\left[\alpha M\left(\Delta_{x} v_{x}+\Delta_{y} v_{y}+\Delta_{z} v_{z}\right)+M\left(\Delta_{x} V_{x}+\Delta_{y} V_{y}+\Delta_{z} V_{z}\right)\right]\right]_{i, j, k}^{n+1 / 2}
$$

In the above equations, the superscripts denote the time step, and the subscripts denote the spatial indices. The symbol $\Delta$ represents the discrete form of the spatial differential operator, for example,

$$
\left.\Delta_{x} v_{x}\right|_{i, j, k}=\frac{27\left(v_{x i+1 / 2, j, k}-v_{x i-1 / 2, j, k}\right)-\left(v_{x^{i+3 / 2, j, k}}-v_{x^{i}-3 / 2, j, k}\right)}{24 h},
$$

where $h$ denotes grid spacing, $\bar{V}$ denotes the arithmetic average in time domain, $\left(V^{n+1 / 2}+V\right.$ $\left.{ }^{n-1 / 2}\right) / 2$ and the coefficients $A, B, C, D, E$ and $F$ are defined as 


$$
\begin{aligned}
A_{x} & =\frac{\bar{m}_{x}}{\left(\bar{m}_{x} \bar{\rho}_{x}-\bar{\rho}_{f_{x}}^{2}\right)}, \\
B_{x} & =\frac{\bar{\rho}_{f_{x}} \bar{b}_{x}}{2\left(\bar{m}_{x} \bar{\rho}_{x}-\bar{\rho}_{f_{x}}^{2}\right)}, \\
C_{x} & =\frac{\bar{\rho}_{f_{x}}}{\left(\bar{m}_{x} \bar{\rho}_{x}-\bar{\rho}_{f_{x}}^{2}\right)}, \\
D_{x} & =\frac{\left(2 \bar{m}_{x} \bar{\rho}_{x}-2 \bar{\rho}_{f_{x}}^{2}-\bar{\rho}_{x} \bar{b}_{x} d t\right)}{\left(2 \bar{m}_{x} \bar{\rho}_{x}-2 \bar{\rho}_{f_{x}}^{2}+\bar{\rho}_{x} \bar{b}_{x} d t\right)}, \\
E_{x} & =\frac{2 \bar{\rho}_{f_{x}}}{\left(2 \bar{m}_{x} \bar{\rho}_{x}-2 \bar{\rho}_{f_{x}}^{2}+\bar{\rho}_{x} \bar{b}_{x} d t\right)}, \\
F_{x} & =\frac{2 \bar{\rho}_{x}}{\left(2 \bar{m}_{x} \bar{\rho}_{x}-2 \bar{\rho}_{f_{x}}^{2}+\bar{\rho}_{x} \bar{b}_{x} d t\right)} .
\end{aligned}
$$

The effective media parameters yield a more accurate representation in the region near interfaces (Graves 1996). The parameters are given by the harmonic average:

$$
\begin{aligned}
& \bar{\rho}_{x}=\left[\frac{1}{2}\left(\frac{1}{\rho_{i, j, k}}+\frac{1}{\rho_{i+1, j, k}}\right)\right]^{-1}, \\
& \bar{\rho}_{y}=\left[\frac{1}{2}\left(\frac{1}{\rho_{i, j, k}}+\frac{1}{\rho_{i, j+1, k}}\right)\right]^{-1}, \\
& \bar{\rho}_{z}=\left[\frac{1}{2}\left(\frac{1}{\rho_{i, j, k}}+\frac{1}{\rho_{i, j, k+1}}\right)\right]^{-1},
\end{aligned}
$$

for the density. Similar averages are used for $m, \rho_{f}$, and $b$. The rigidity $\mu$ is given by

$$
\begin{aligned}
& \bar{\mu}_{x y}=\left[\frac{1}{4}\left(\frac{1}{\mu_{i, j, k}}+\frac{1}{\mu_{i+1, j, k}}+\frac{1}{\mu_{i, j+1, k}}+\frac{1}{\mu_{i+1, j+1, k}}\right)\right]^{-1}, \\
& \bar{\mu}_{x z}=\left[\frac{1}{4}\left(\frac{1}{\mu_{i, j, k}}+\frac{1}{\mu_{i+1, j, k}}+\frac{1}{\mu_{i, j, k+1}}+\frac{1}{\mu_{i+1, j, k+1}}\right)\right]^{-1}, \\
& \bar{\mu}_{y z}=\left[\frac{1}{4}\left(\frac{1}{\mu_{i, j, k}}+\frac{1}{\mu_{i, j+1, k}}+\frac{1}{\mu_{i, j, k+1}}+\frac{1}{\mu_{i, j+1, k+1}}\right)\right]^{-1} .
\end{aligned}
$$

\section{Free surface boundary condition}

The free surface boundary condition in the staggered-grid scheme is easily implemented by explicitly satisfying the zero-stress condition (Graves 1996; Kristek et al. 2002) assuming pore fluid pressure at the free surface vanishes (Zhu and McMechan 1991): 
$\tau_{z z}=\tau_{x z}=\tau_{y z}=p=\left.0\right|_{z=0}$.

Lavender (1988) and Graves (1996) used imaged values of the stress components above the free surface based on their anti-symmetry condition about the free surface. Since, however, this stress-imaging method degrades accuracy of the fourth order FD formulation to the second order level, the adjusted FD approximations are used for the free surface boundary condition (Kristek et al. 2002).

\section{E Absorbing boundary condition}

Chew and Liu (1996) showed the effectiveness of the PML as an absorbing boundary condition for elastic waves. Using the concept of complex coordinates (Chew and Weedon 1994) in the frequency domain with a time dependence of $e^{-i \omega t}$, the complex coordinate stretching variables can be written as

$\tilde{x}_{i}=\int_{0}^{x_{i}} s_{i}\left(x_{i}^{\prime}\right) d x_{i}^{\prime}, s_{i}\left(x_{i}^{\prime}\right)=a_{i}^{s}+i \omega_{i}^{s} / \omega$,

where $a_{i}^{s} \geq 1$ is a scaling factor and $\omega_{i}^{s} \geq 0$ is an attenuation factor. The derivative $\partial / \partial \widetilde{x}_{i}$ can be expressed in terms of the regular coordinate stretching variables, $\partial / \partial \widetilde{x}_{i}=1 / s_{i} \partial / \partial x_{i}$ In the PML regin and the frequency domain, eqs. (IV.7)-(IV.10) become

$$
\begin{aligned}
& (-i \omega)\left(m \rho-\rho_{f}^{2}\right) \hat{v}_{i}=m \hat{\tau}_{i j, \tilde{j}}+\rho_{f} b \hat{V}_{i}+\rho_{f} \hat{p}_{, \tilde{i}}, \\
& (-i \omega)\left(m \rho-\rho_{f}^{2}\right) \hat{V}_{i}=-\rho_{f} \hat{\tau}_{i j, \tilde{j}}-\rho b \hat{V}_{i}-\rho \hat{p}_{, \tilde{i}}, \\
& (-i \omega) \hat{\tau}_{i j}=\mu\left(\hat{v}_{i, \widetilde{j}}+\hat{v}_{j, \tilde{i}}\right)+\delta_{i j}\left(\lambda \hat{v}_{k, \widetilde{k}}+\alpha M \hat{V}_{k, \widetilde{k}}\right), \\
& \hat{p}=-\alpha M \hat{v}_{k, \tilde{k}}-M \hat{V}_{k, \tilde{k}},
\end{aligned}
$$

where the hat refers to the frequency domain. The regular coordinate variable $x_{i}$ is replaced by the complex coordinate stretching variable $\tilde{x}_{i}$,

$$
\begin{aligned}
& (-i \omega)\left(m \rho-\rho_{f}^{2}\right) \hat{v}_{i}=\frac{m}{s_{j}} \hat{\tau}_{i j, j}+\rho_{f} b \hat{V}_{i}+\frac{\rho_{f}}{s_{i}} \hat{p}_{, i}, \\
& (-i \omega)\left(m \rho-\rho_{f}^{2}\right) \hat{V}_{i}=-\frac{\rho_{f}}{s_{j}} \hat{\tau}_{i j, j}-\rho b \hat{V}_{i}-\frac{\rho}{s_{i}} \hat{p}_{, i},
\end{aligned}
$$




$$
\begin{aligned}
& (-i \omega) \hat{\tau}_{i j}=\mu\left(\frac{\hat{v}_{i, j}}{s_{j}}+\frac{\hat{v}_{j, i}}{s_{i}}\right)+\frac{\delta_{i j}}{s_{k}}\left(\lambda_{c} \hat{v}_{k, k}+\alpha M \hat{V}_{k, k}\right), \\
& \hat{p}=-\frac{\alpha M}{s_{k}} \hat{v}_{k, k}-\frac{M}{s_{k}} \hat{V}_{k, k},
\end{aligned}
$$

To simplify the PML equations, the field variables are split as $v_{j}=v_{j}^{k}$ and $\tau_{i j}=\tau_{i j}^{k}$, where the superscript denotes the splitting direction. For example, eq. (IV.34) for $x$ component can be written as,

$$
\begin{aligned}
& (-i \omega)\left(m \rho-\rho_{f}^{2}\right) \hat{v}_{x}^{(x)}=\frac{m}{s_{x}} \hat{\tau}_{x x, x}+\rho_{f} b \hat{V}_{x}^{(x)}+\frac{\rho_{f}}{s_{x}} \hat{p}_{, x}, \\
& (-i \omega)\left(m \rho-\rho_{f}^{2}\right) \hat{v}_{x}^{(y)}=\frac{m}{s_{y}} \hat{\tau}_{x y, y}+\rho_{f} b \hat{V}_{x}^{(y)} \\
& (-i \omega)\left(m \rho-\rho_{f}^{2}\right) \hat{v}_{x}^{(z)}=\frac{m}{s_{z}} \hat{\tau}_{x z, z}+\rho_{f} b \hat{V}_{x}^{(z)}
\end{aligned}
$$

By taking the inverse Fourier transform, the PML equations in the time domain are obtained:

$$
\begin{aligned}
& \left(m \rho-\rho_{f}^{2}\right)\left(a_{x}^{s} \partial_{t}+\omega_{x}^{s}\right) v_{x}^{(x)}=m \tau_{x x, x}+\rho_{f} b\left(a_{x}^{s} V_{x}^{(x)}+\omega_{x}^{s} \int_{-\infty}^{t} V_{x}^{(x)} d t^{\prime}\right)+\rho_{f} p_{, x}, \\
& \left(m \rho-\rho_{f}^{2}\right)\left(a_{y}^{s} \partial_{t}+\omega_{y}^{s}\right) v_{x}^{(y)}=m \tau_{x y, y}+\rho_{f} b\left(a_{y}^{s} V_{x}^{(y)}+\omega_{y}^{s} \int_{-\infty}^{t} V_{x}^{(y)} d t^{\prime}\right) \\
& \left(m \rho-\rho_{f}^{2}\right)\left(a_{z}^{s} \partial_{t}+\omega_{z}^{s}\right) v_{x}^{(z)}=m \tau_{x z, z}+\rho_{f} b\left(a_{z}^{s} V_{x}^{(z)}+\omega_{z}^{s} \int_{-\infty}^{t} V_{x}^{(z)} d t^{\prime}\right) .
\end{aligned}
$$

Eqs. (IV.35)-(IV.37) can be transformed similarly.

To incorporate the PML boundary condition, the computational domain is divided into a PML region and an interior region. The outgoing waves are absorbed by the PML via high attenuation of the outgoing waves. Even though a perfectly matched interface generates no reflections at the interface, it cannot completely exclude reflections in discretized media due to discretization errors. The discretization error which generates fictitious reflection from outgoing waves is proportional to the grid spacing and the contrast between the two media (Chew and Jin 1996). It has been known that the first few PMLs generate the most significant reflections, which forces the increments in attenuation properties in the first few PMLs to be small. On the contrary, insufficient attenuation may also cause reflections from the computational boundary, i.e., the outer boundary of the PML region. Therefore, the 
attenuation must be increased significantly toward the end of the PML region to guarantee the absorption of the outgoing waves.

In this work, the scaling factor and the attenuation factor have the following forms $a_{i}^{s}=1+a_{\max }\left(l_{i} / L_{\mathbf{P M L}}\right)^{n}$, $\omega_{i}^{s}=2 \pi f_{0} \omega_{\max }\left(l_{i} / L_{\mathbf{P M L}}\right)^{n+\alpha}$,

where $l_{i}$ is the distance from the interface between the PML region and the interior region, $L_{\mathrm{PML}}$ is the thickness of the PML region, $a_{\max }$ and $\omega_{\max }$ are empirical coefficients and $f_{0}$ is the dominant frequency of the source. $a_{\max }$ and $\omega_{\max }$ control the rate of attenuation of the outgoing waves. Different rates of change of $a_{i}^{s}$ and $\omega^{s}{ }_{i}$ can lead to significant improvement of the PML performance (Rickard et al. 2003). In the numerical examples, we used 10 layers of PML to minimize the reflection from the PML region. We have found that outgoing waves can most efficiently be absorbed with $a_{\max }=3.5, \omega_{\max }=11, n=2$ and $\alpha=3$.

\section{F $\quad$ Source implementations}

We use the first time derivative of the Gaussian function is used as the source time function:

$F(t)=\left(t-t_{0}\right) e^{-\left[\pi f_{0}\left(t-t_{0}\right)\right]^{2}}$,

where $t_{0}$ is the time delay and $f_{0}$ is the dominant frequency. Since we are concerned with a composite material, a bulk source is used (Zhu and McMechan 1991). The explosive source is partitioned linearly between the two phases by multiplying the source function by factors $W_{s}=(1-\phi)$, $W_{f}=\phi$,

where $W_{s}$ and $W_{f}$ are the weighting factor for the solid normal stresses and fluid pressure, respectively, and $\phi$ is the porosity.

\section{G Numerical examples: Homogeneous model}

A homogeneous porous medium is used to examine the accuracy of our numerical solutions and the performance of the PML method. The results are compared to the analytical solution given by Dai et. al (1995). The pore fluid is considered as an ideal inviscid fluid, and other properties of the medium are those of gas saturated sandstone provided in Table IV.1. The size of the model is $N_{x} \times N_{y} \times N_{z}=160 \times 160 \times 160$ nodes with 10 grids of PML on all sides of the computational boundary. The spatial and temporal increments are $2 \mathrm{~m}$ and $0.4 \mathrm{~ms}$, 
respectively. A point source with a dominant frequency of $15 \mathrm{~Hz}$ and a delay time of $150 \mathrm{~ms}$ is located at the center of the domain and a receiver is located below the source (Fig. IV.3). The vertical component of the solid velocity at the receiver is illustrated in Fig. IV.4 showing a very good agreement between numerical and analytical values. The snapshots of the vertical component of the solid velocity at $t=24 \mathrm{~ms}$ and $t=32 \mathrm{~ms}$ are shown in Fig. IV.5. At $t=24 \mathrm{~ms}$, the $\mathrm{P}$ wave reaches the computational boundary. At $t=32 \mathrm{~ms}$, the $\mathrm{P}$ wave mostly disappears as a result of the PML but the slow P wave propagates inside the medium. The lack of reflections from the computational boundary shows the effectiveness of the PML method.

\section{H Numerical Examples: Two layer model}

In order to compare poroelastic wave propagation and elastic wave propagation, Dutta and Odé's gas-water contact model (1983) is used. This model has the same rock matrix in both gas and water saturated regions. The upper layer is gas saturated sandstone whereas the lower layer is water saturated sandstone whose parameters are given in Table IV.1, except that permeability is increased to $10^{-6} \mathrm{~m}^{2}$ to highlight the behavior of the slow $\mathrm{P}$ wave. The parameters for the elastic modeling are listed in Table IV.2. The time increment is 0.0005 ms whereas the size of the model is $N_{x} \times N_{y} \times N_{z}=300 \times 200 \times 500$ nodes with $0.25 \mathrm{~cm}$ spatial separation. The interface is located at $1.0 \mathrm{~m}$ depth. A point source with a dominant frequency of $10,000 \mathrm{~Hz}$ and a delay time of $0.2 \mathrm{~ms}$ is located at $0.5 \mathrm{~m}$ depth. This domain is also enclosed by the PML boundary.

Fig. IV.6 shows the snapshots of the vertical component of the solid velocity (Figs. IV.6a-6c) and the velocity of the fluid relative to the solid (Figs. IV.6d-6f), defined as $\mathbf{w}$ in eq. (IV.1) and eq. (IV.2). In this figure, the symbols P and P S denote the P wave and the slow $\mathrm{P}$ wave, respectively, and the subscripts indicate the associated layer. Multiple symbols represent reflected and transmitted waves. The snapshots at $t=0.54 \mathrm{~ms}$ (Figs. IV.6a and IV.6d) show that the solid and the fluid motions are in phase for the $\mathrm{P}$ wave and out of phase for the slow $\mathrm{P}$ wave. Incident $\mathrm{P}$ wave generates the reflected $\mathrm{P} \quad 1 \mathrm{P} \quad 1$ and $\mathrm{P} 1 \mathrm{P} S 1$, and the transmitted $\mathrm{P} 1 \mathrm{P} 2$ and $\mathrm{P} 1 \mathrm{PS} 2$. At $t=1.06 \mathrm{~ms}$, the slow $\mathrm{P}$ wave generates the reflected PS $1 \mathrm{P} 1$ and PS 1 PS 1 , and the transmitted PS $1 \mathrm{P} 2$ and PS $1 \mathrm{PS} 2$. The snapshots at $t=1.5 \mathrm{~ms}$ show that the slow $\mathrm{P}$ wave in the lower layer is attenuated more than the slow $\mathrm{P}$ wave in the upper layer due to the higher fluid viscosity in the lower layer. 
To compare the poroelastic wave propagation with the elastic wave propagation qualitatively, elastic wave propagation is also simulated for the same geometry and source. Fig. IV.7 shows snapshots of the poroelastic and the elastic wave propagation at $t=0.44 \mathrm{~ms}$. Figs. IV.7a and IV.7b are the snapshots from the vertical component of the solid and the relative velocity for the poroelastic case whereas Fig. IV.7c shows the vertical velocity component for the elastic case. The mode converted slow P waves (P 1 P S 1 and P 1 P S 2 ) are shown in Figs. IV.7a and IV.7b. Dutta and Odé (1983) showed that fluid flow results in the mode conversion and hence contributes to inelastic absorption of seismic energy. This results in the decrease of the reflection and transmission coefficients. Fig. IV.8 shows the cross lines from Figs. IV.7a and IV.7c. As expected, the amplitudes of the reflected and the transmitted waves in the poroelastic medium are less than that in the elastic medium. However, the mode converted slow $\mathrm{P}$ waves are generated in the poroelastic medium.

\section{Numerical Examples: Transition layer model}

In this example, layered models with a transition zone from a dry layer to a fully saturated one are considered. Seismic velocity depends significantly on fluid phase composition. The $\mathrm{P}$ wave velocity in dry medium is relatively low and decreases slightly as the saturation increases. Above 95\% saturation, the $\mathrm{P}$ wave velocity increases abruptly to the $\mathrm{P}$ wave velocity at full saturation (Tuncay and Corapcioglu 1996; Bachrach and Nur 1998; Bradford 2002).

Physical properties of the model are given in Table IV.3. Van Genuchten's (1980) closed form expressions for the capillary pressure-saturation relations are used to obtain a realistic water saturation profile. The effective medium parameters for the partially saturated media are computed via Bachrach and Nur (1998). A point source with a dominant frequency of $1,200 \mathrm{~Hz}$ and a delay time of $2 \mathrm{~ms}$, and 101 receivers with a spacing of $40 \mathrm{~cm}$ are located at the free surface.

Synthetic seismograms are shown in Fig. IV.9. The fully saturated layer is located $18 \mathrm{~m}$ below the surface. Fig. IV.9a is obtained from using a transition layer of $15 \mathrm{~m}$ whereas Fig. IV.9b is obtained with the sharp interface. The reflections $\left(\mathrm{P}_{1} \mathrm{P}_{1}\right)$ in Fig. IV.9a are delayed more than those in Fig. IV.9b, demonstrating the presence of a low velocity layer caused by 
the partial saturation, as suggested by previous studies (Tuncay and Corapcioglu 1996; Bachrach and Nur 1998; Bradford 2002).

\section{J Numerical Examples: Anticline model}

An anticline model (Fig. IV.10) whose physical properties are given in Table IV.1 is used to study 3-D poroelastic wave propagation in heterogeneous media. The size of the model is $N_{x}$ $\times N_{y} \times N_{z}=300 \times 500 \times 200$ nodes. The spatial and temporal increments are $0.4 \mathrm{~m}$ and 0.08 $\mathrm{ms}$, respectively. A point source with a dominant frequency of $150 \mathrm{~Hz}$ and a delay time of $20 \mathrm{~ms}$, and 80 receivers are located at the free surface with $2 \mathrm{~m}$ spacing (Fig. IV.10a). Figs. IV.10b and IV.10c show the cross-section of the $y-z$ and $x-z$ planes. The matrix of the anticline is sandstone and contains gas, oil and water. The saturated anticline sandstone reservoir is enclosed by water saturated shale (Fig. IV.10b). Each interface is denoted by A, B, C and D (Fig. IV.10c).

Fig. IV.11 shows synthetic seismograms from the vertical and horizontal component of the solid velocity. Direct P waves and Rayleigh waves indicated as (1) and (2), respectively, are dominant in the seismograms. Unlike previous simulations no slow P wave is observed. This is because the slow P wave is highly dissipative at low frequencies (Dutta and Odé 1983). Reflections from interfaces are also present. (3) and (7) denote $\mathrm{P}$ and $\mathrm{S}$ wave reflections from the interface A, respectively. Interfaces B, C, and D also generate P wave reflections (4), (5), and (6). Because of the low contrast in physical properties at interface $\mathrm{D}$, reflection (6) is weaker than other reflections. S wave reflections from interface A and B are shown as (7) and (8), respectively. (9) and (10) indicate converted S waves from interface A and B. Multiple reflections denoted by (11) and (12) can also be observed.

\section{K Summary}

In the present work, the velocity-stress staggered-grid FD method with the PML ABC is developed to simulate wave propagation in 3-D heterogeneous poroelastic media. The PML boundary condition which attenuates outgoing seismic waves is used to simulate unbounded media. With a number of numerical experiments, we have shown that poroelastic wave phenomena in unbounded heterogeneous media can be simulated accurately and efficiently with this method. In the numerical simulations of the Biot's equations, the slow $\mathrm{P}$ wave is apparent for very high permeability matrices and/or very low viscosity fluids. In 
sedimentary basins, the equilibration of pore fluid pressure between the fine layers can produce significant attenuation (Pride et al., 2002). Thus, the comprehensive study of poroelastic wave propagation will produce a methodology for the seismic analysis of reservoir characterization, fluid detection and recovery monitoring. To simulate more realistic seismic wave propagation, we plan to include the effects of inelasticity, anisotropy, and fluid compostion in our future work. 
Table IV.1 Physical properties of the porous media (from Özdenvar and McMechan 1997)

\begin{tabular}{lccccc}
\hline & Shale & Sandstone & Water & Gas & Oil \\
\hline$K_{b}\left(\mathrm{~N} / \mathrm{m}^{2}\right)$ & $1.60 \times 10^{9}$ & $1.70 \times 10^{9}$ & - & - & - \\
$\mu\left(\mathrm{N} / \mathrm{m}^{2}\right)$ & $1.70 \times 10^{9}$ & $1.855 \times 10^{9}$ & - & - & - \\
$K_{s}, K_{f}\left(\mathrm{~N} / \mathrm{m}^{2}\right)$ & $11.2 \times 10^{9}$ & $35.0 \times 10^{9}$ & $2.4 \times 10^{9}$ & $0.022 \times 10^{9}$ & $1.855 \times 10^{9}$ \\
$\rho_{s}, \rho_{f}\left(\mathrm{~kg} / \mathrm{m}^{3}\right)$ & $2.65 \times 10^{3}$ & $2.65 \times 10^{3}$ & $1.0 \times 10^{3}$ & $0.1 \times 10^{3}$ & $0.88 \times 10^{3}$ \\
$\phi$ & 0.11 & 0.30 & - & - & - \\
$\kappa\left(\mathrm{m}^{2}\right)$ & $10^{-12}$ & $10^{-12}$ & - & - & - \\
$a$ & 3.0 & 2.0 & - & - & - \\
$\eta\left(\mathrm{Nsm}^{-2}\right)$ & - & - & $1.0 \times 10^{-3}$ & $1.50 \times 10^{-5}$ & $1.80 \times 10^{-1}$ \\
\hline
\end{tabular}

Table IV.2 Physical properties of saturated rock (obtained from Table IV.1)

\begin{tabular}{lcc}
\hline & Gas Saturated Sandstone & Water Saturated Sandstone \\
\hline$\rho\left(\mathrm{kg} / \mathrm{m}^{3}\right)$ & $1.885 \times 10^{3}$ & $2.155 \times 10^{3}$ \\
$V_{p}(\mathrm{~m} / \mathrm{s})$ & $1.503 \times 10^{3}$ & $2.216 \times 10^{3}$ \\
$V_{s}(\mathrm{~m} / \mathrm{s})$ & $0.995 \times 10^{3}$ & $0.962 \times 10^{3}$ \\
\hline
\end{tabular}

Table IV.3 Physical properties of the porous media.

\begin{tabular}{lccc}
\hline & Sandstone & Water & Air \\
\hline$K_{b}\left(\mathrm{~N} / \mathrm{m}^{2}\right)$ & $1.70 \times 10^{9}$ & - & - \\
$\mu\left(\mathrm{N} / \mathrm{m}^{2}\right)$ & $1.855 \times 10^{9}$ & - & - \\
$K_{s}, K_{f}\left(\mathrm{~N} / \mathrm{m}^{2}\right)$ & $35.0 \times 10^{9}$ & $2.4 \times 10^{9}$ & $1.0 \times 10^{5}$ \\
$\rho_{s}, \rho_{f}\left(\mathrm{~kg} / \mathrm{m}^{3}\right)$ & $2.65 \times 10^{3}$ & $1.0 \times 10^{3}$ & 1.3 \\
$\phi$ & 0.50 & - & - \\
$\kappa\left(\mathrm{m}^{2}\right)$ & $10^{-12}$ & - & - \\
$a$ & 2.0 & - & - \\
$\eta\left(\mathrm{Nsm}^{-2}\right)$ & - & $1.0 \times 10^{-3}$ & $1.8 \times 10^{-5}$ \\
\hline
\end{tabular}




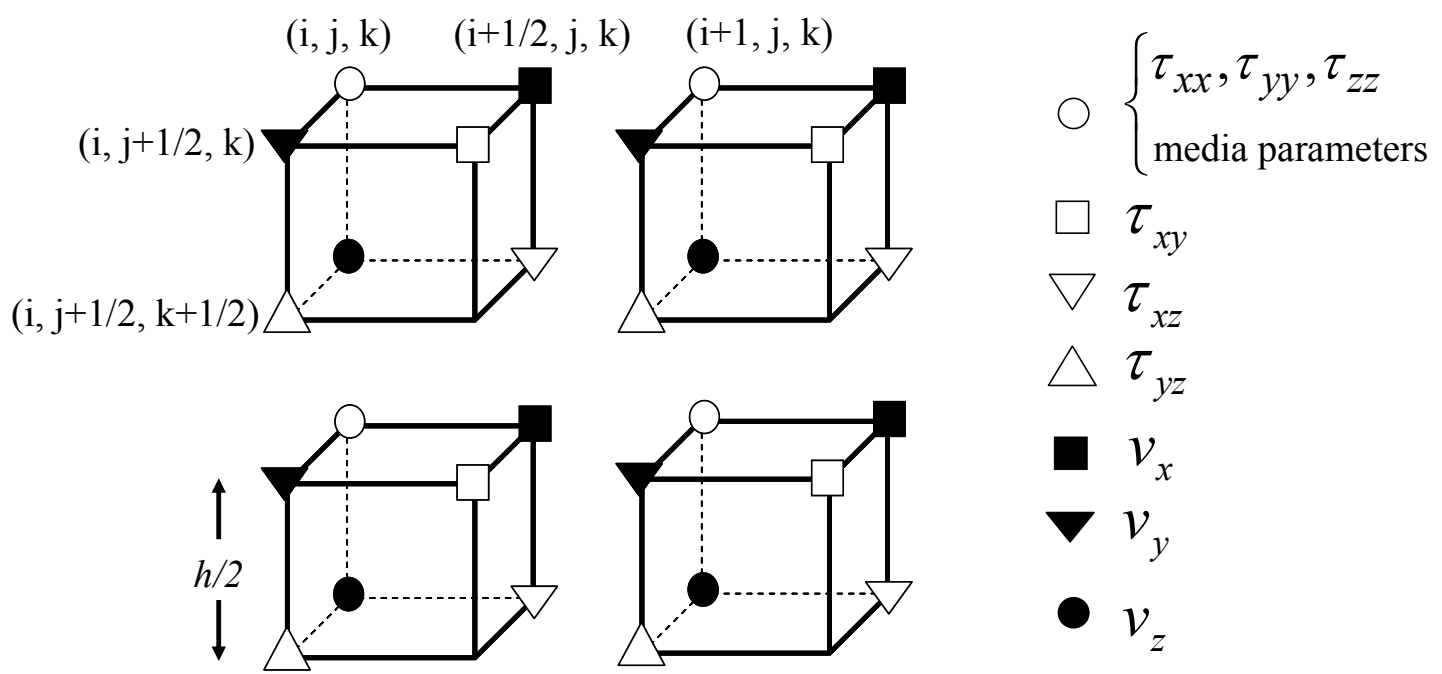

Fig. IV.1 Grid layout for staggered grid formulation. The indices (i, j, k) represent the spatial coordinates (x, y, $\mathrm{z}$ ), respectively, and the grid spacing $h$ is defined as the length between the centers of two adjacent grid cells (modified from Graves 1996). 


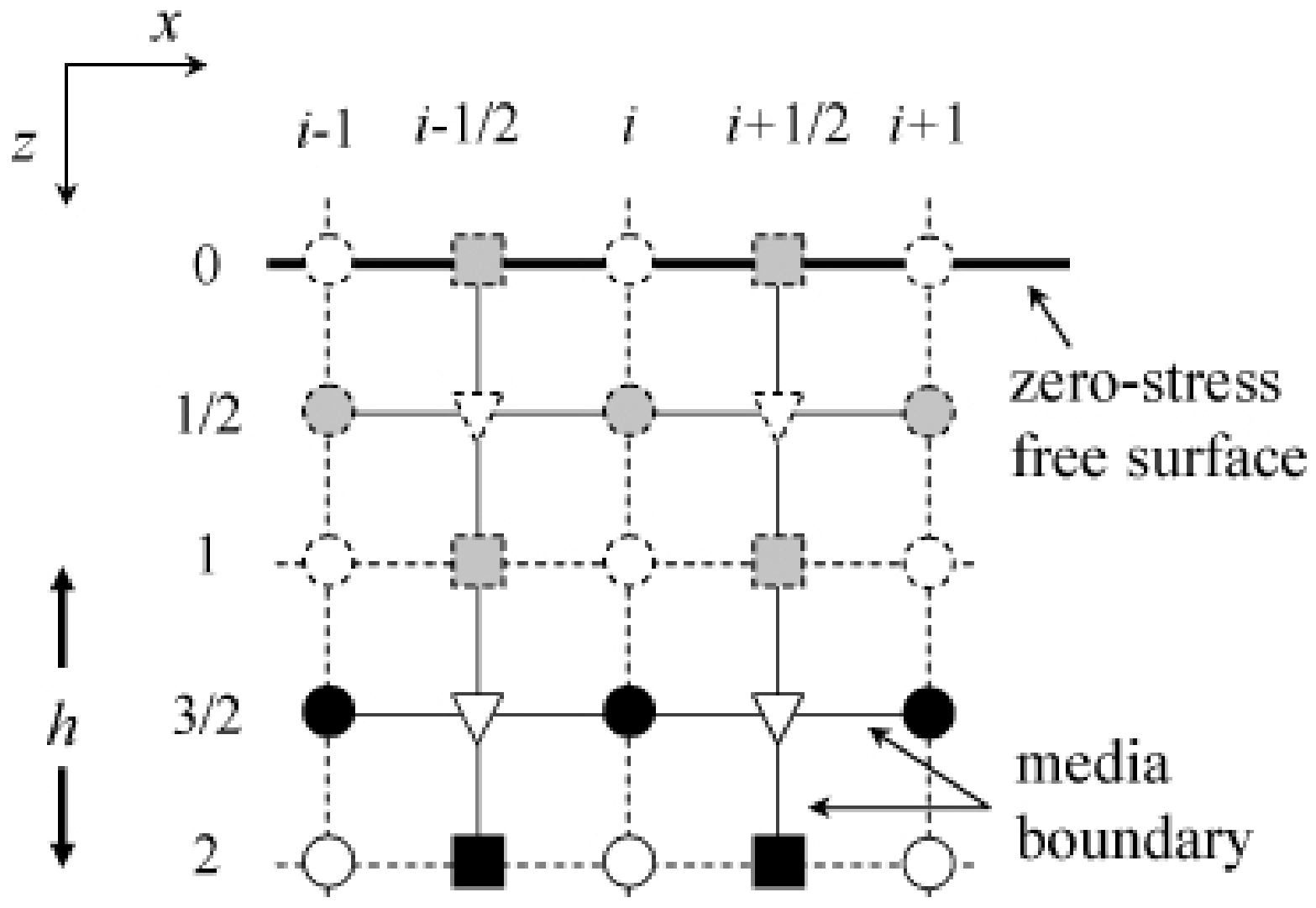

Fig. IV.2 Layout of the free surface boundary. The location of the zero-stress free surface is shown by the thick solid line and is coincident with the normal stress node locations. Dotted nodes are obtained from the adjusted FD approximations (Kristek et al. 2002). 


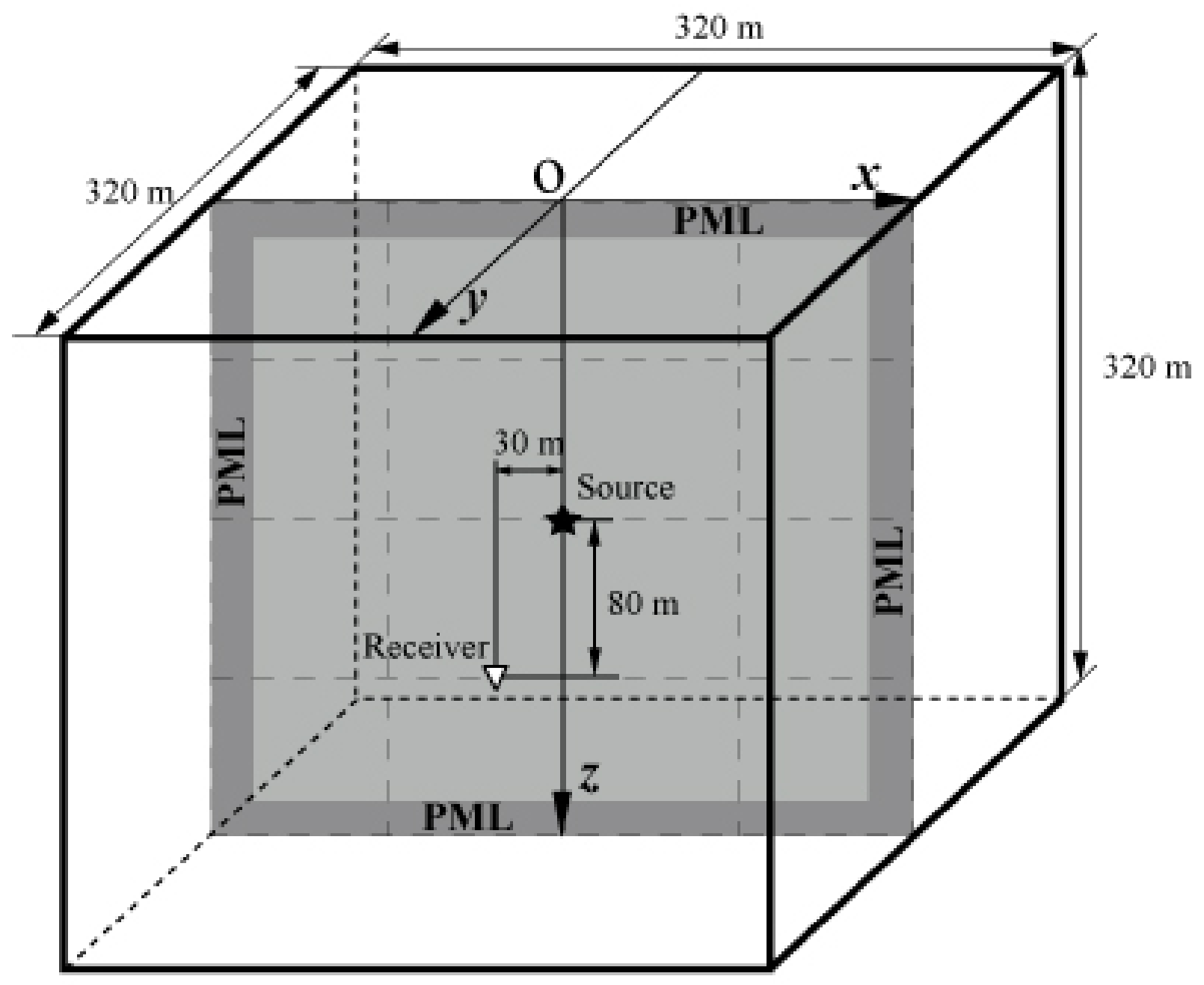

Fig. IV.3 Schematic diagram of a homogeneous model. The PML is applied to all sides of computational boundary. 


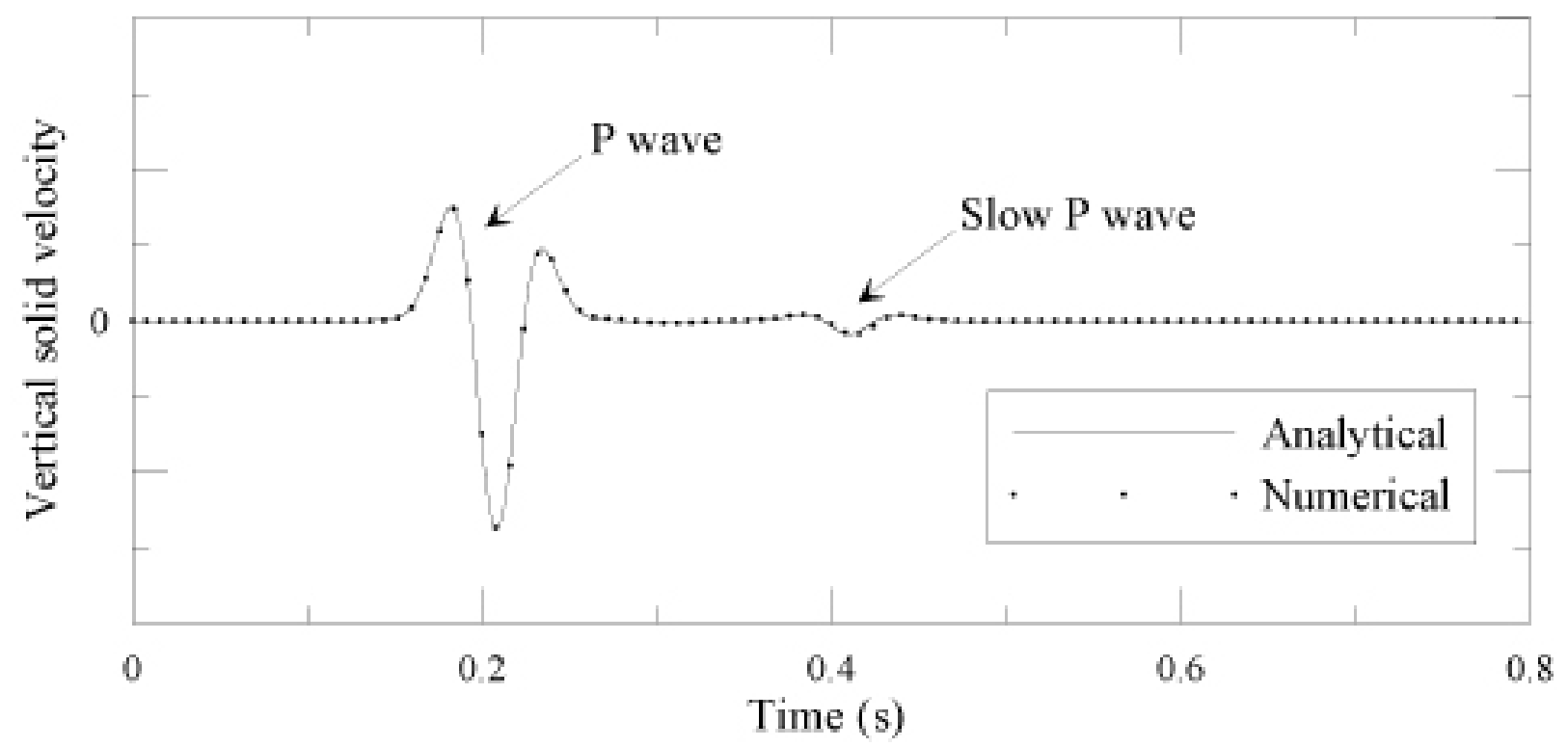

Fig. IV.4 Comparison of numerical and analytical waveforms of the vertical component of the solid velocity for an explosive source in an infinite homogeneous medium (Fig. IV.3). 


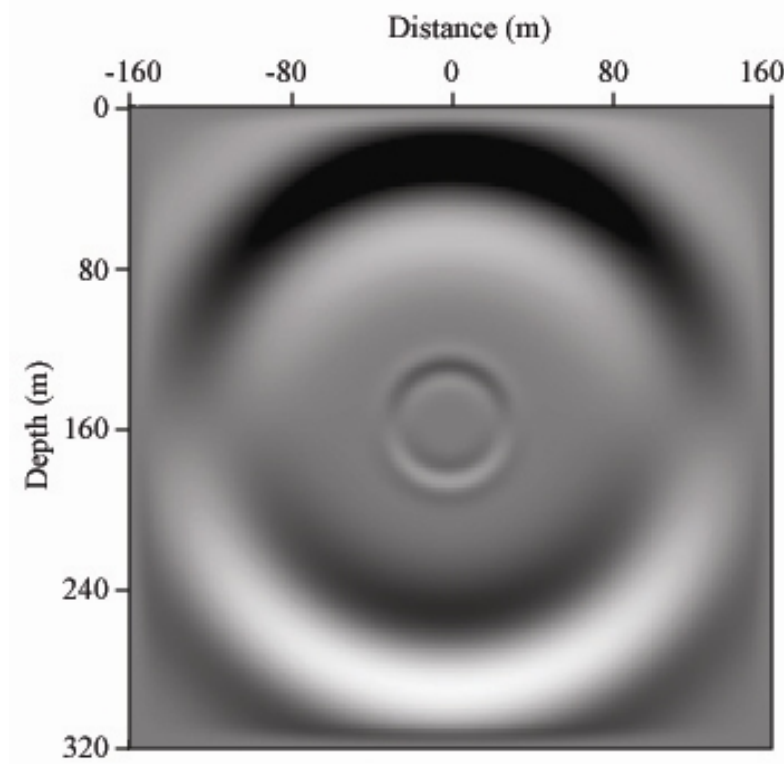

(a)

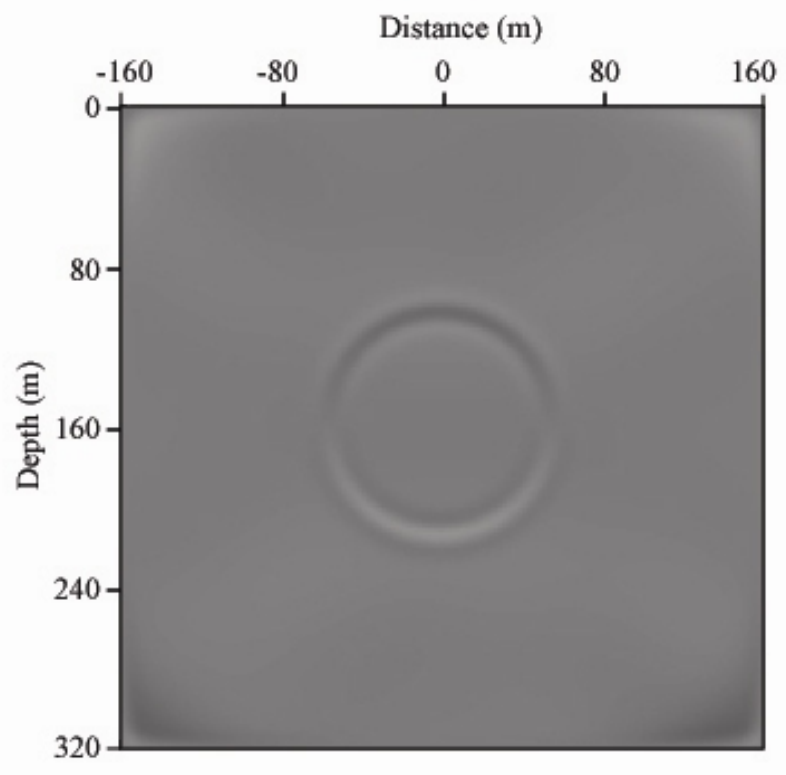

(b)

Fig. IV.5 Snapshots of the vertical component of the solid velocity at $t=0.24 \mathrm{~s} \mathrm{(a)} \mathrm{and} t=0.32 \mathrm{~s}(\mathrm{~b})$ in the $x z$ plane of Fig. IV.3. 


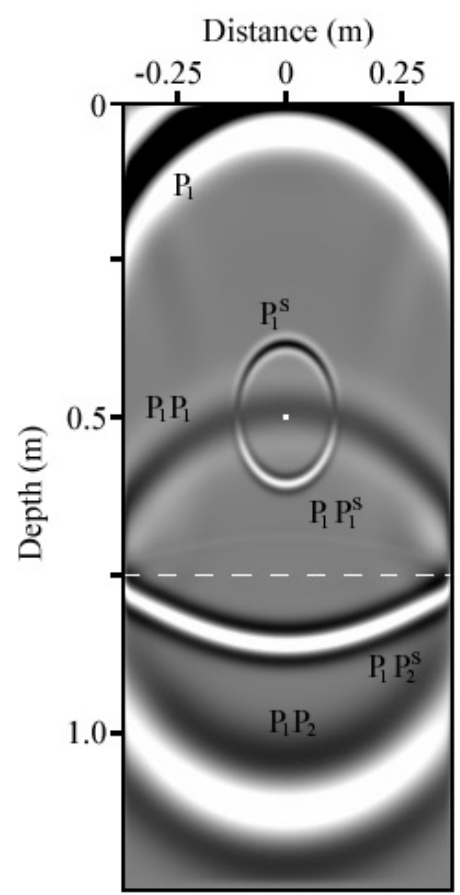

(a)

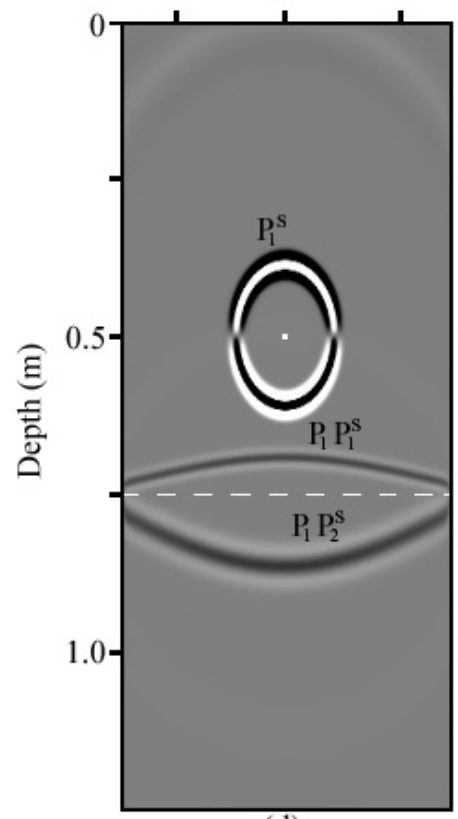

(d)

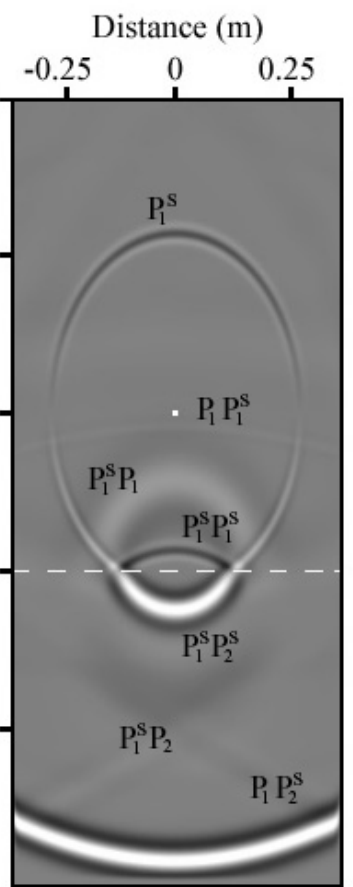

(b)

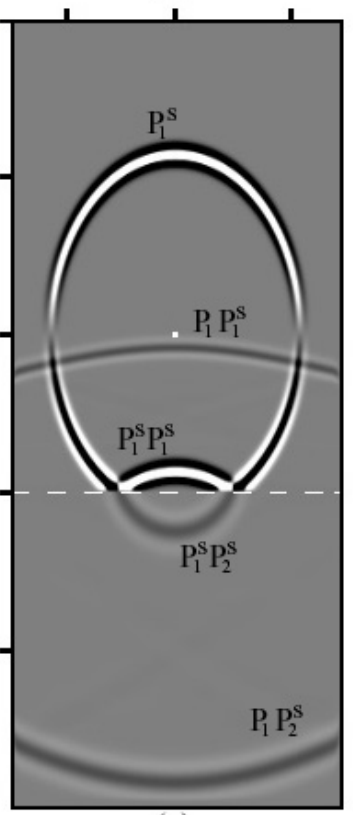

(e)

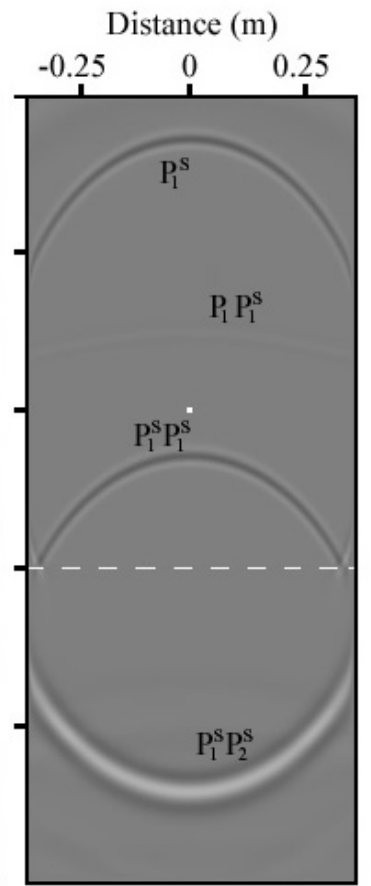

(c)

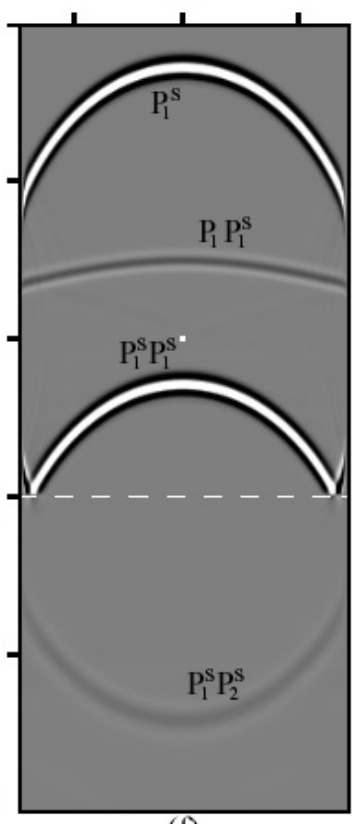

(f)

Fig. IV.6 Snapshots $(t=0.54,1.06$, and $1.5 \mathrm{~ms})$ of the vertical component of the solid (a)-(c) and relative (d)(e) velocities from two layer model. The dotted line and the white point show the interface and the seismic source, respectively. The scaling of amplitudes for (a)-(c) is five times that for (d)-(f). 


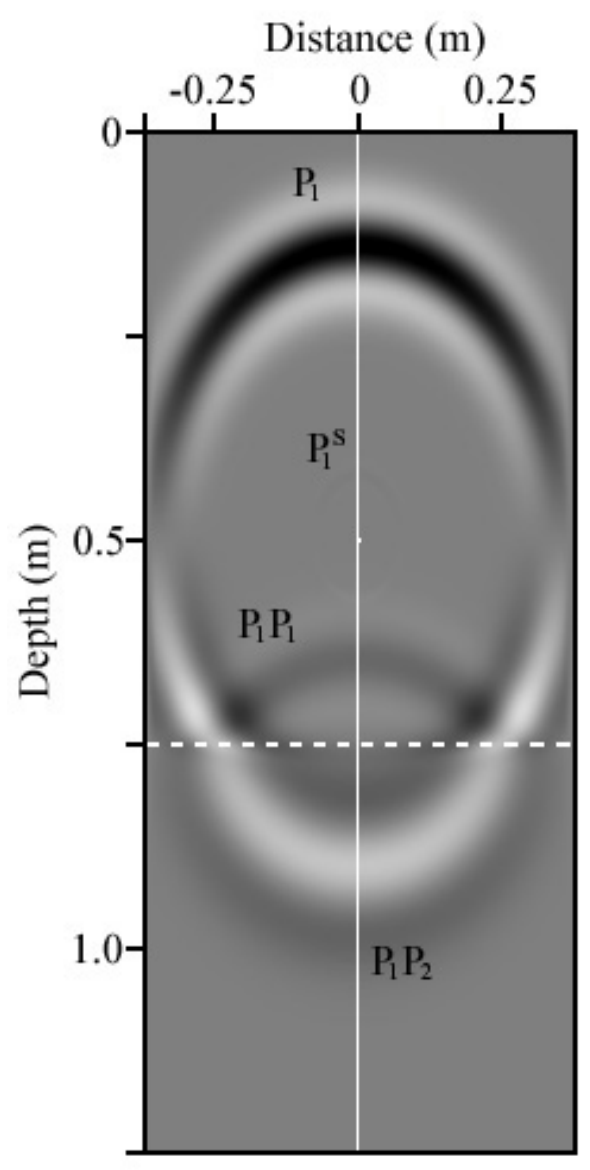

(a)

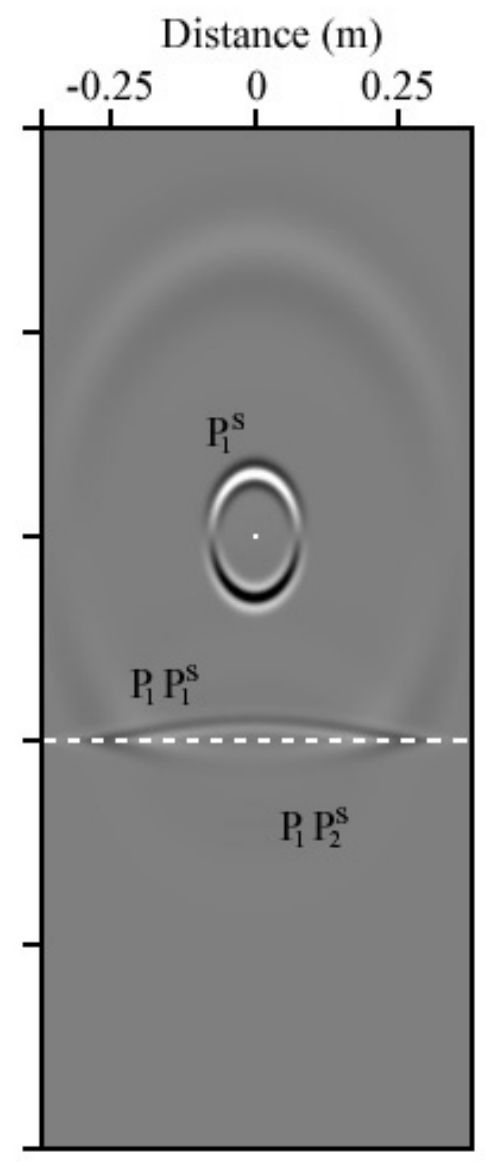

(b)

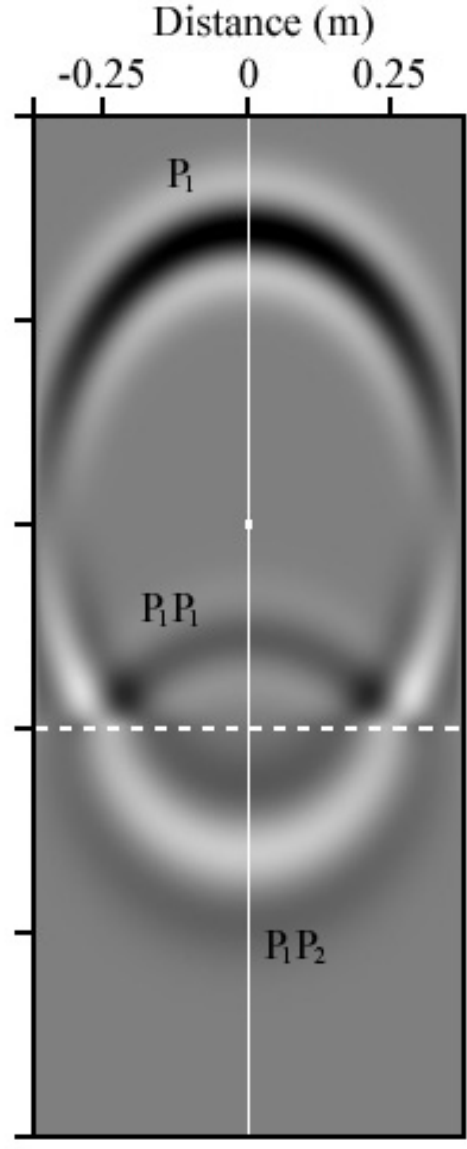

(c)

Fig. IV.7 Snapshots of the vertical component of the solid (a) and relative (b) velocities for the poroelastic case and the vertical component of the solid velocity (c) for the elastic case at $t=0.44 \mathrm{~ms}$. The dotted line and the white point show the interface and the seismic source, respectively. The solid white lines denote cross lines for Fig. 8. All snapshots have the same scaling. 


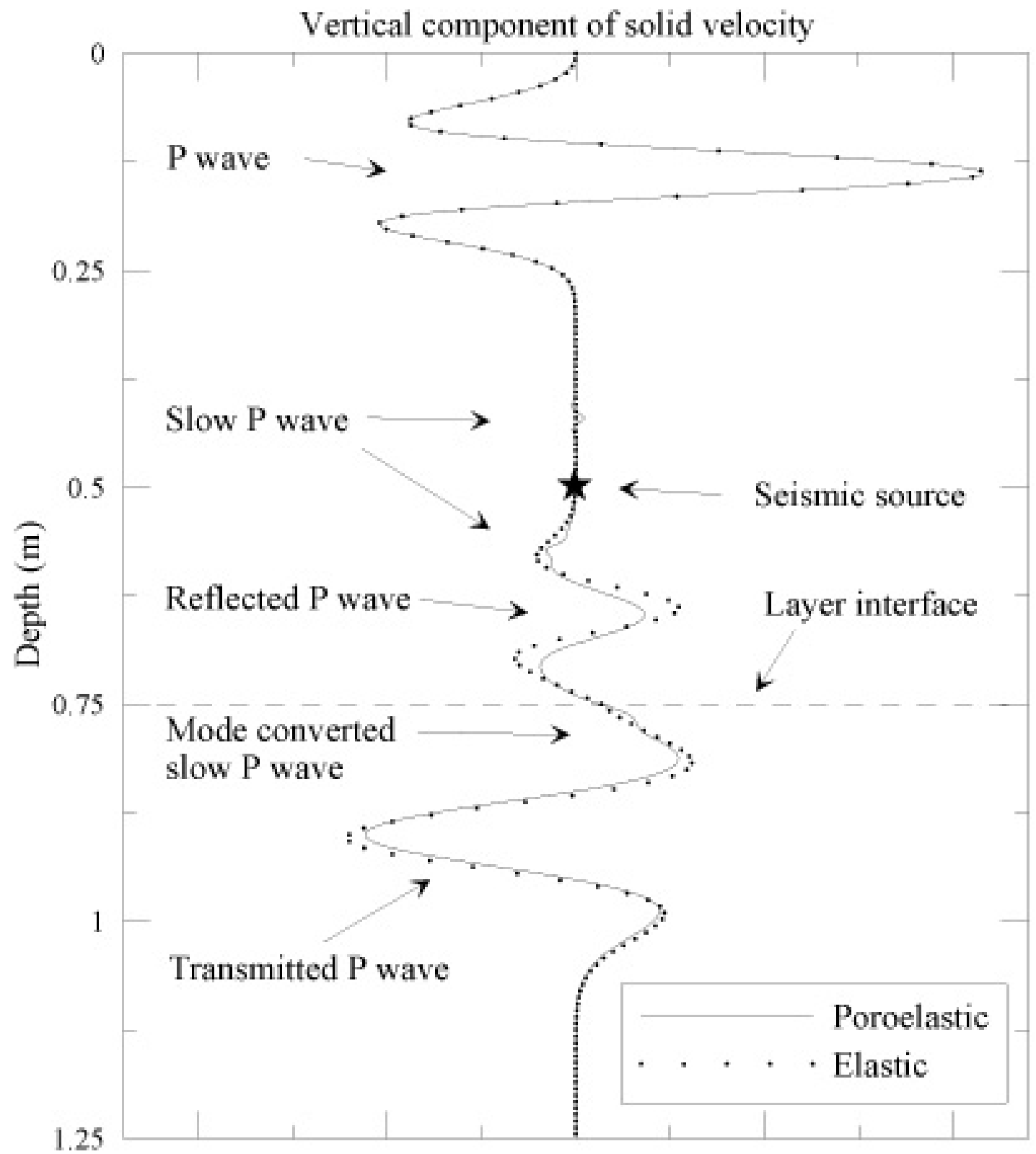

Fig. IV.8 The amplitude of the vertical component of the solid velocity from cross lines shown in Fig. IV.7. 


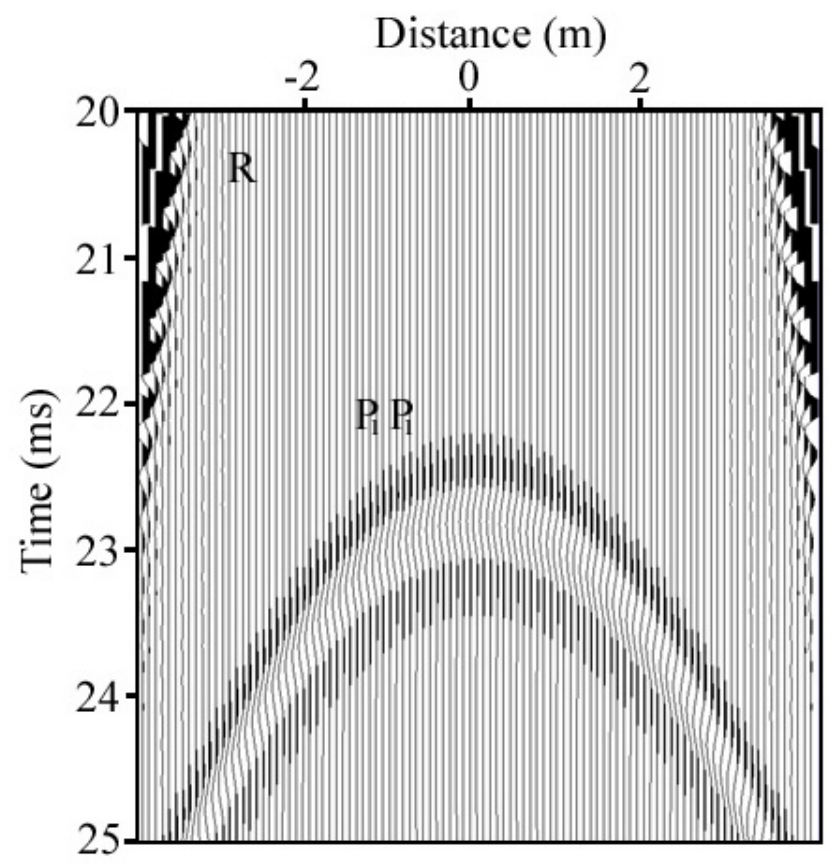

(a)

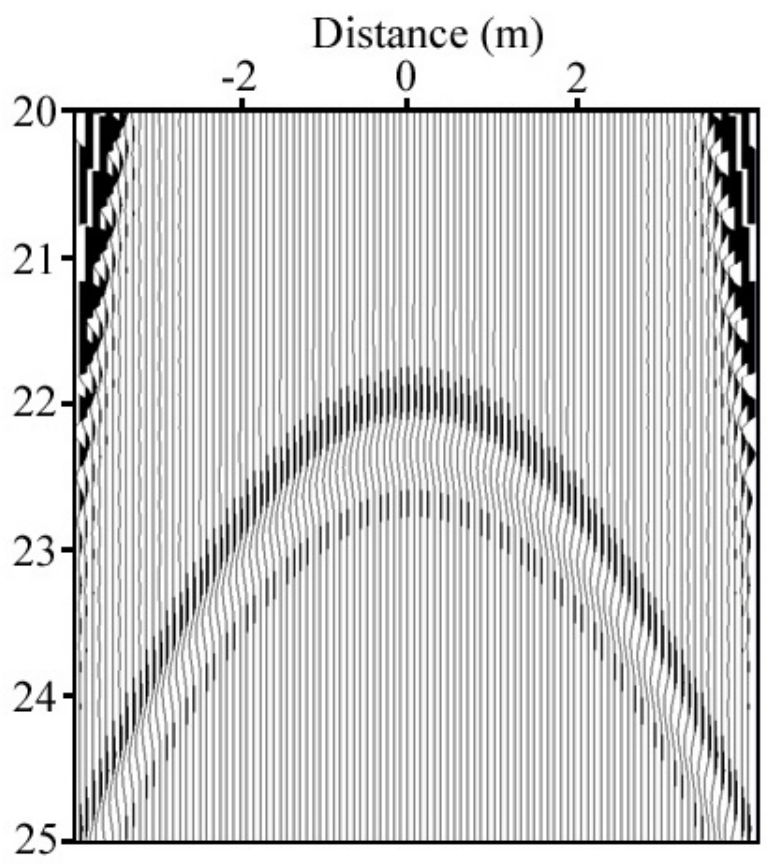

(b)

Fig. IV.9 Synthetic seismograms from the transition layer model. The water table is located $18 \mathrm{~m}$ below the free surface. The thicknesses of the transition layers are $15 \mathrm{~m}$ (a) and $0 \mathrm{~m}(\mathrm{~b}) . \mathrm{R}$ and $\mathrm{P}_{1} \mathrm{P}_{1}$ show the Rayleigh wave and reflection from the water table, respectively. 


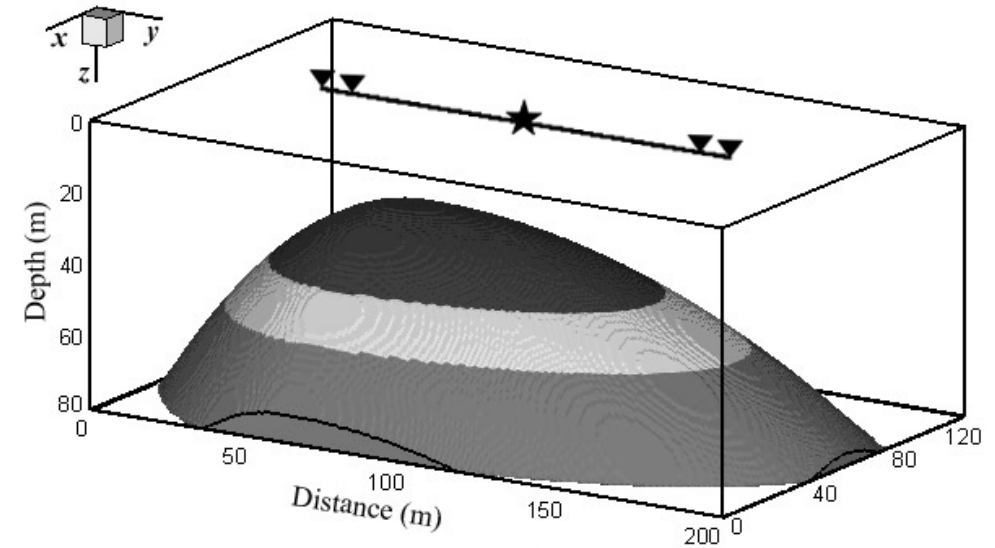

(a)
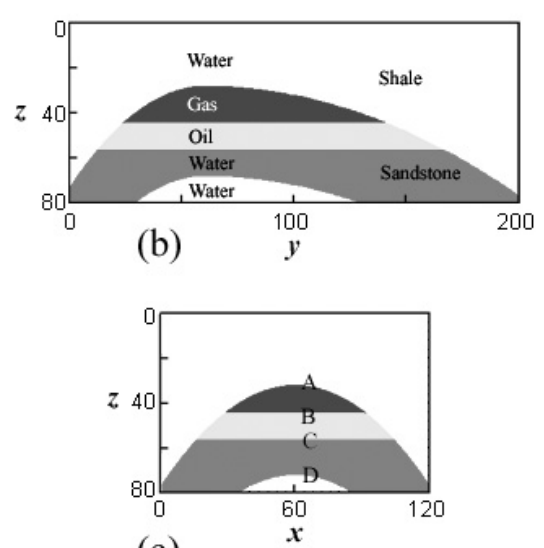

(c)

Fig. IV.10 Geometry of the 3-D anticline model and source/receiver configuration. Physical parameters of each layer are given in Table 1. (a) Source and receivers are located at surface. Star and triangles denote the seismic source and receivers, respectively. 81 receivers with $2 \mathrm{~m}$ spacing are used for recording synthetic seismogram. (b) A saturated anticline sandstone reservoir enclosed by water saturated shale. (c) Interfaces are labeled A, B, C, and D. 


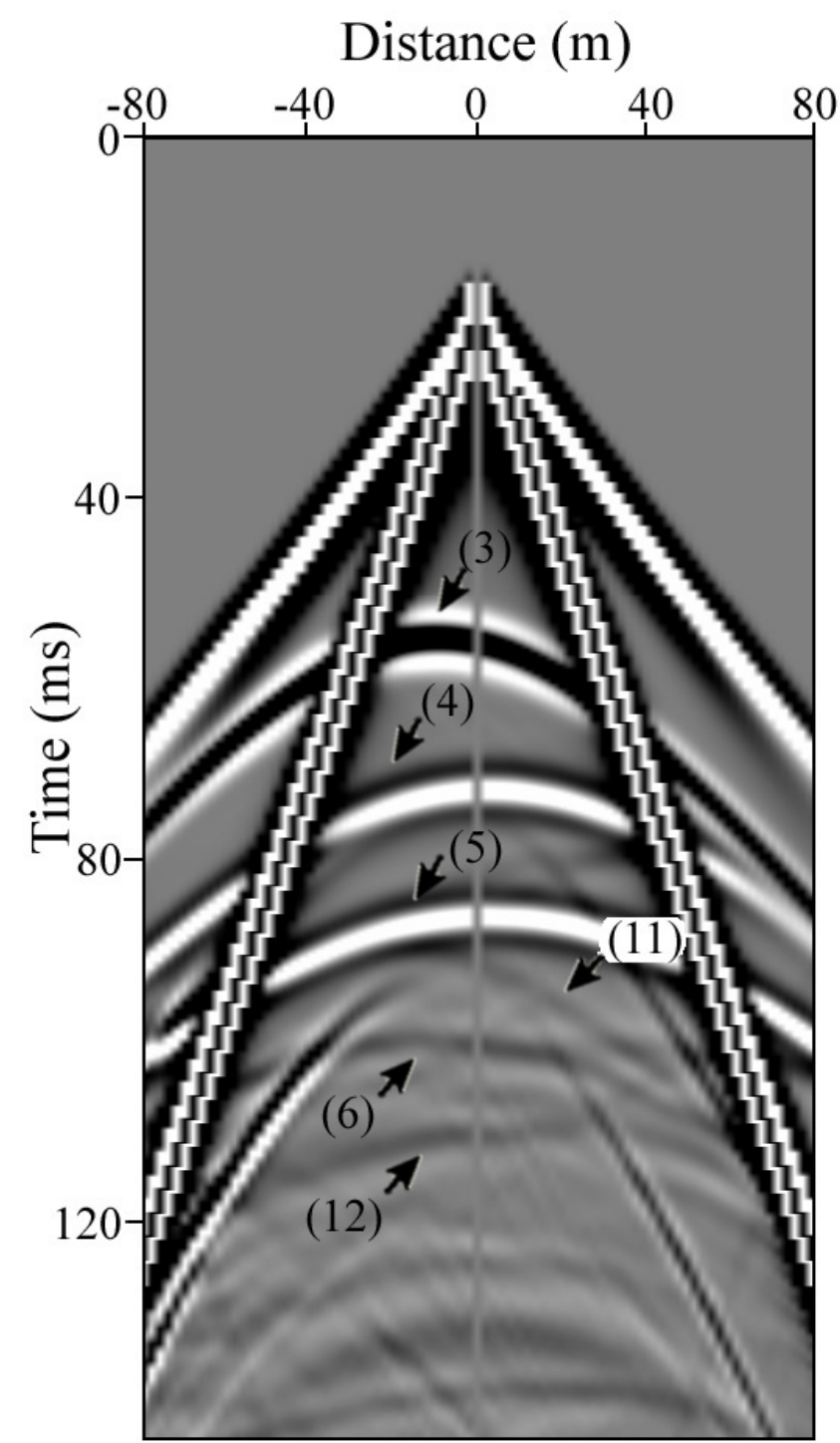

(a)

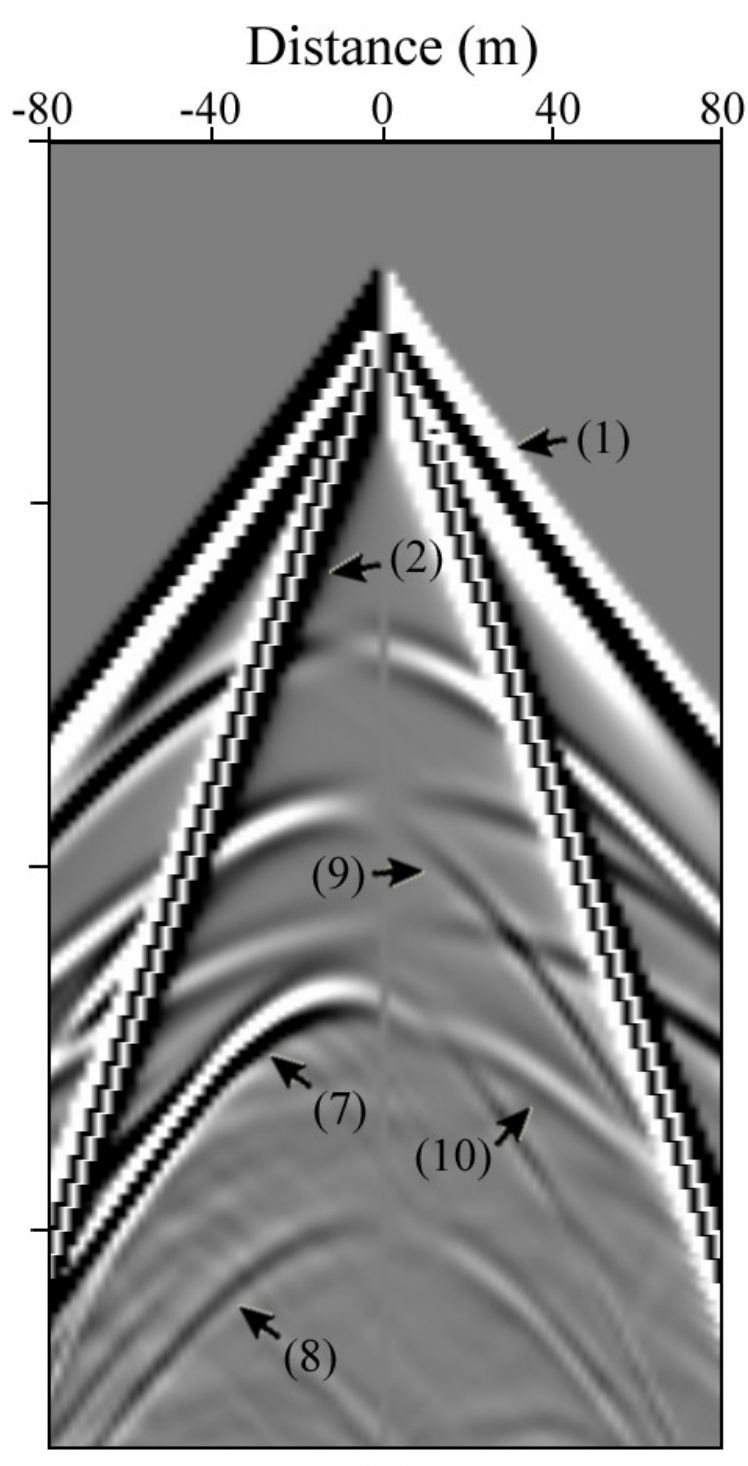

(b)

Fig. IV.11 Synthetic seismograms from the vertical component (a) and horizontal component (b) of the solid velocity at the free surface for the 3-D anticline model shown in Fig. IV.10. Labeling of arrivals are indicated for (1) direct P wave, (2) Rayleigh wave, (3) P wave reflection from interface A, (4) P wave reflection from interface $B$, (5) $P$ wave reflection from interface $C$, (6) $P$ wave reflection from interface $D$, (7) $S$ wave reflection from interface $\mathrm{A}$, (8) S wave reflection from interface B, (9) PS reflection from interface A, (10) PS reflection from interface $\mathrm{B}$, and (11) and (12) $\mathrm{P}$ wave multiple reflections. 


\section{Calibrate 1D Simulations (Subtask 4.4)}

\section{A Strategy for Phase II}

In the re-evaluation of our Phase II strategy we decided that most of the effort should be placed on the seismic inversion facets of the project. This was in keeping with the emphasis on remote detection, the possibility of using well data to replace the basin simulations, and the fact that we had already demonstrated our basin simulation approach in Phase I. This strategy allowed us to develop and implement the revolutionary inversion approach described in Chapter VI.

Having tested both the basin modeling and calibration at single wells (i.e. one dimensional model) and our seismic inversion technology, we will be able to carry out a full demonstration in Phase III wherein basin simulation and seismic inversion will be integrated and applied through our MAGI system to Illinois Basin data. Results described in Chapter VII are also based on Illinois Basin data but are shown as a test of our seismic inversion technology only. Our basin modeling approach is described in the next section.

\section{B Modeling Approach}

Basin RTM is a product of over 120 man-years of effort in the development of physicochemical models, rate laws, numerical algorithms, visualization, and user interface. At present, it is used by LCG for its basin research into basin processes and to assist clients in their E\&P activities. We expect to release Basin RTM to other academic and industry users in the near future. Basin RTM arises out of the work of Geochemical Research Associates (Bloomington, Indiana) and LCG's collaboration with the petroleum industry and the U.S. Department of Energy, the Gas Research Institute and the U.S. Environmental Protection Agency.

The model we have developed (Ortoleva et al. 1997; Ortoleva 1998; Tuncay, Park, and Ortoleva 2000a,b; Tuncay and Ortoleva 2001; Tuncay, Khalil, and Ortoleva 2001) uses variables that describe small-scale features (grain-grain connectivity; local grain size, shape and mineralogy; porosity; pore fluid composition and phase; and fracture characteristics) within each computational subvolume (finite element). These are assumed to vary continuously across the system. Variations of these variables locate and characterize faults and associated fractures, fluid compartments, and other features. Our model shows how 
these features emerge and change in $3 \mathrm{D}$ by solving partial differential equations for all these variables.

An overview of our Basin RTM simulator is as follows. A complex network of geochemical reactions, fluid and energy transport and rock mechanical (RTM) processes underlies the genesis, dynamics and present-day characteristics of petroleum reservoirs or other crustal phenomena in Basin RTM (see Fig. V.1). Basin RTM integrates most relevant geological factors and RTM processes believed to operate in a sedimentary basin. As reservoirs are fundamentally 3-D in nature, Basin RTM is based on 3-D finite element simulation techniques.

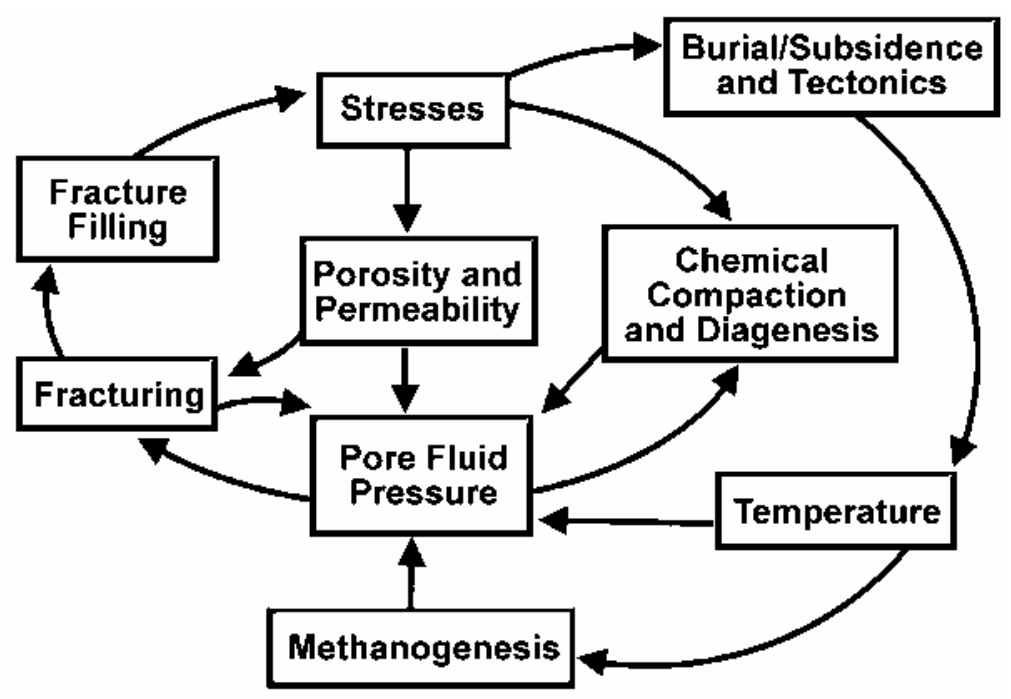

Fig. V.1 Complex network of coupled processes underlying the dynamics of a sedimentary basin. These factors and their coupling are accounted for in our unique basin simulator. 


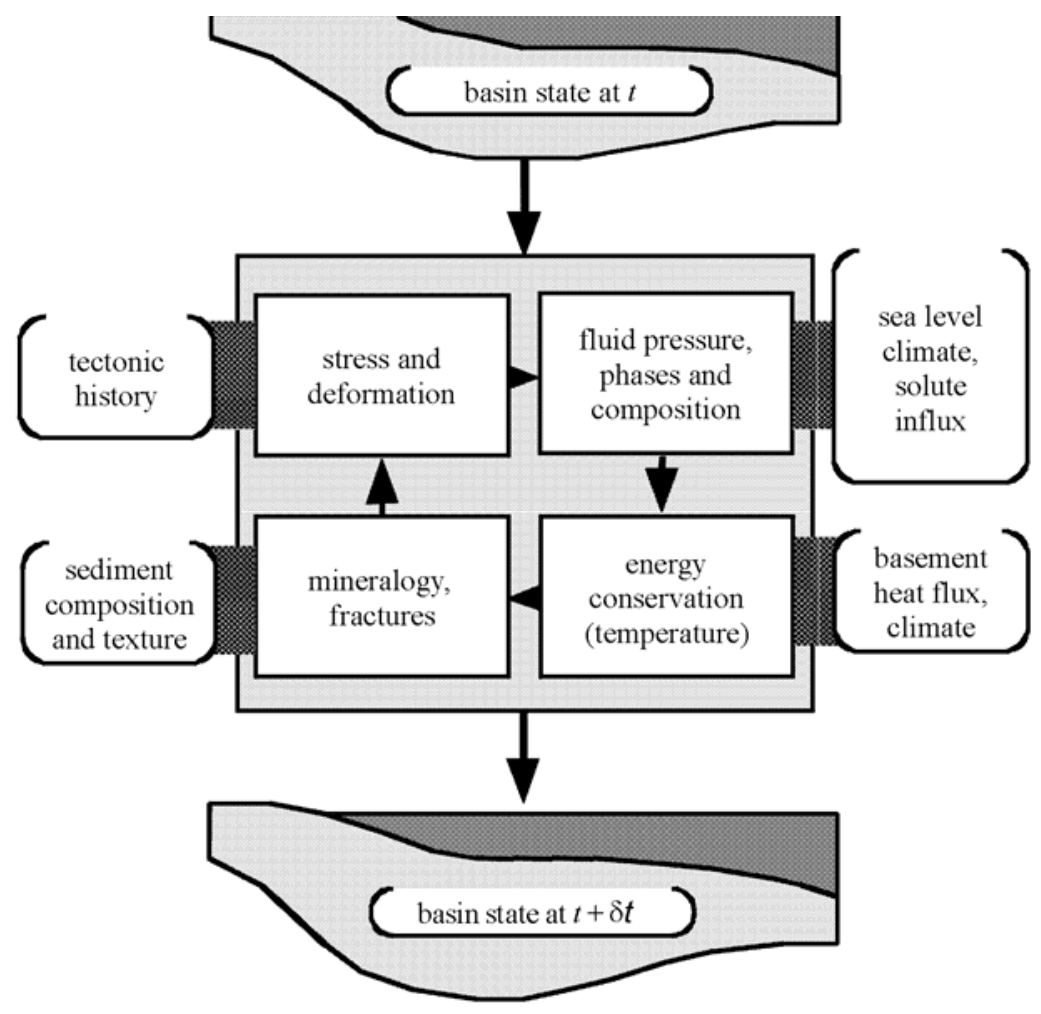

Fig. V.2 Schematic Basin RTM flow chart showing the interplay of geological data and the internal RTM processes in evolving a basin over one computational time step.

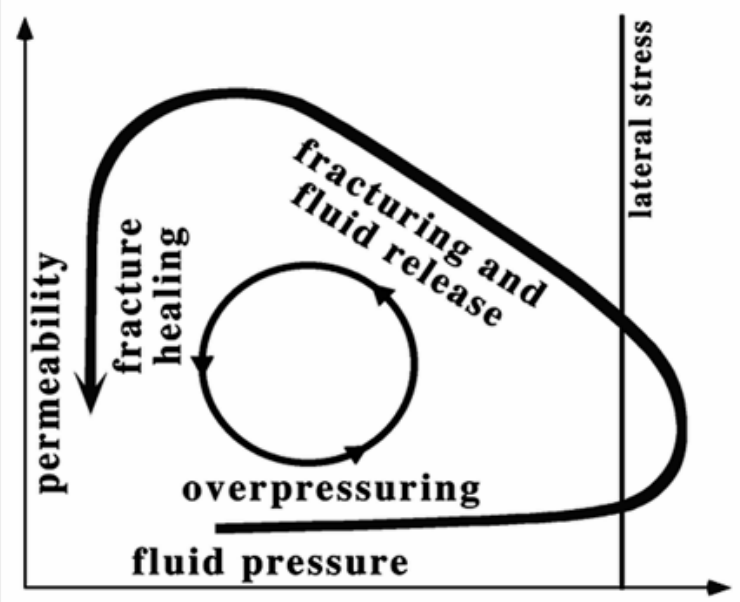

Fig. V.3 Fluid pressuring, fracturing and fracture healing feedback cycle, one example of the many feedback mechanisms inherent in the RTM process network. This cycle can repeat many times during a basin's evolution when conditions are appropriate. 
The RTM processes and geological factors accounted for in Basin RTM are outlined in Fig. V.2. External influences such as sediment input, erosion, sea level, thermal and tectonic effects are allowed to influence the progress of internal RTM processes. Within the basin, these RTM processes modify the sediment chemically and mechanically to arrive at petroleum and mineral reserves, seals, compartments, faults and other internal features.

Basin RTM provides a platform for integrating all available geological data as suggested in Fig. V.2 using the framework provided by the laws of physics and chemistry to facilitate exploration or field development. Available information can be divided into geological data and the physico-chemical rate laws. The former make a simulation tailored to a specific basin. The physico-chemical information gives Basin RTM the power to predict resource location and characteristics and other features of the evolving basin.

Basin RTM can be used to carry out sensitivity analyses or to identify new phenomena such as self-organization and other nonlinear effects that can dramatically affect the disposition of reservoirs in a basin (Ortoleva 1990, 1994). Basin RTM simulations show that the sedimentary basin or other crustal system is highly dynamic, exhibiting a strong degree of autonomy, rather than simply responding to the details of the external influences. As Basin RTM uses the laws of physics and chemistry to extrapolate data on present-day location and characteristics of lithologies beyond the locations of this data, it enhances the use and interpretation of seismic, well log, surface geological and other data

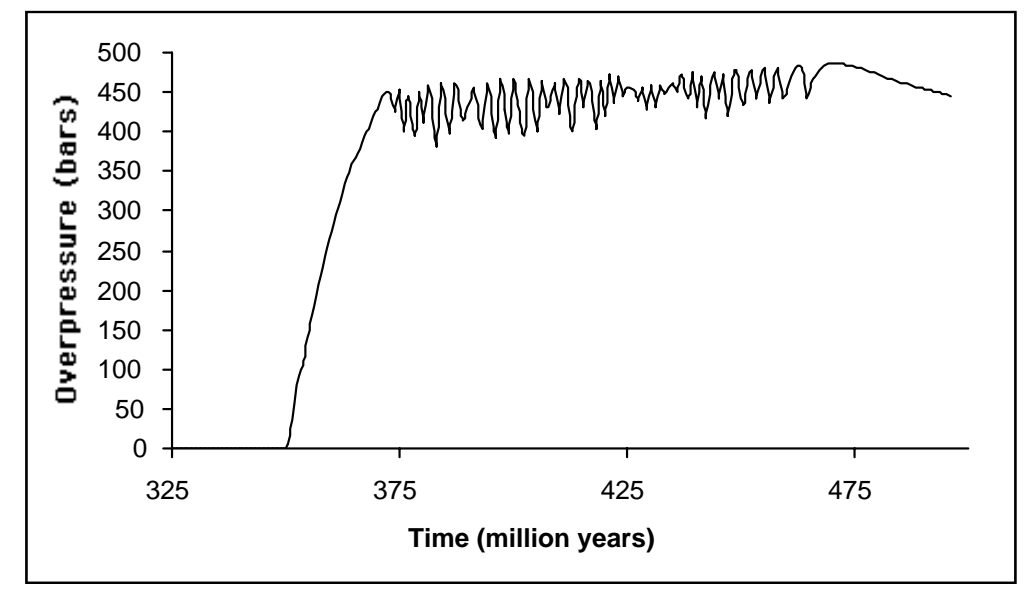

Fig. V.4 Basin RTM predicted overpressure evolution at the bottom of the Ellenburger Formation. Overpressuring starts around 350 million years into the simulation, when fractures in the layer above the source rock disappear. Oscillatory behavior is a result of cyclic fracturing of the seal driven by petroleum generation. After $470 \mathrm{My}$ the cyclic petroleum expulsion ceases and the pressure, oil saturation fracturing and other variables show a more steady behavior (Tuncay, Park and Ortoleva 2000a). 
in defining the present-day and historical state of the crust. Basin RTM can be used to identify windows of time during which formations along a proposed migration pathway were open, and not blocked due to compaction, fracture closure or diagenetic cementation. Alternatively, Basin RTM can predict if and when a seal was breached and hydrocarbons escaped through natural fracturing or permeability-enhancing diagenetic reactions (see Figs. V.3-V.5).

Basin RTM makes its predictions based on the numerical solution of a set of multiphase, organic and inorganic, reaction-transport equations and equations of rock deformation and heat transfer. Calculations of all effects are done self-consistently to preserve cross-couplings between processes (see Fig. V.1). For example, the determination of temperature is affected by transport, which is affected by changes of porosity that evolves due to temperature-dependent reaction rates. Similarly, the rate of kerogen decomposition depends on temperature which, in turn, depends on thermal transport that is affected, through fluid buoyancy, thermal conductivity, capillarity and relative permeability, by the content of organic material and its thermal decay products. All such coupling relations between the full set of RTM processes as in Fig. V.1 are accounted for in our Basin RTM simulator. 
a)
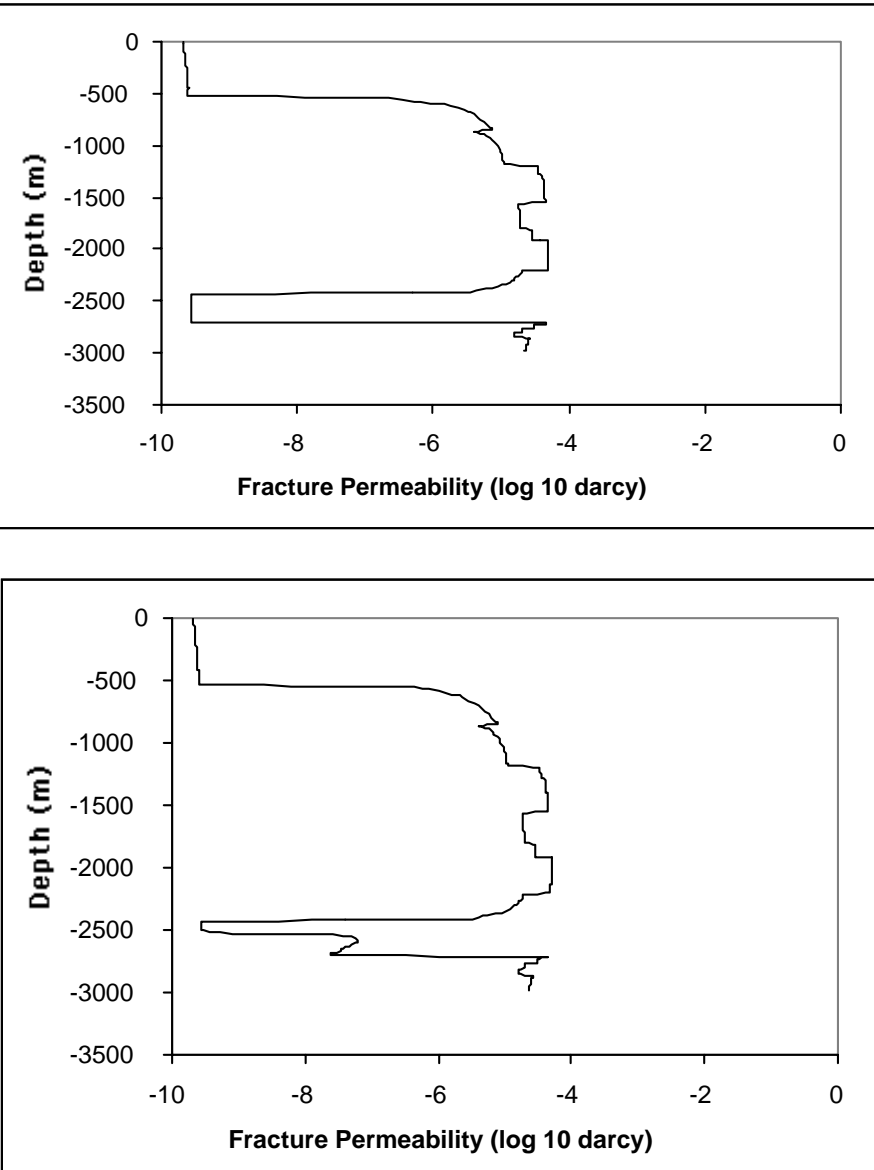

b)

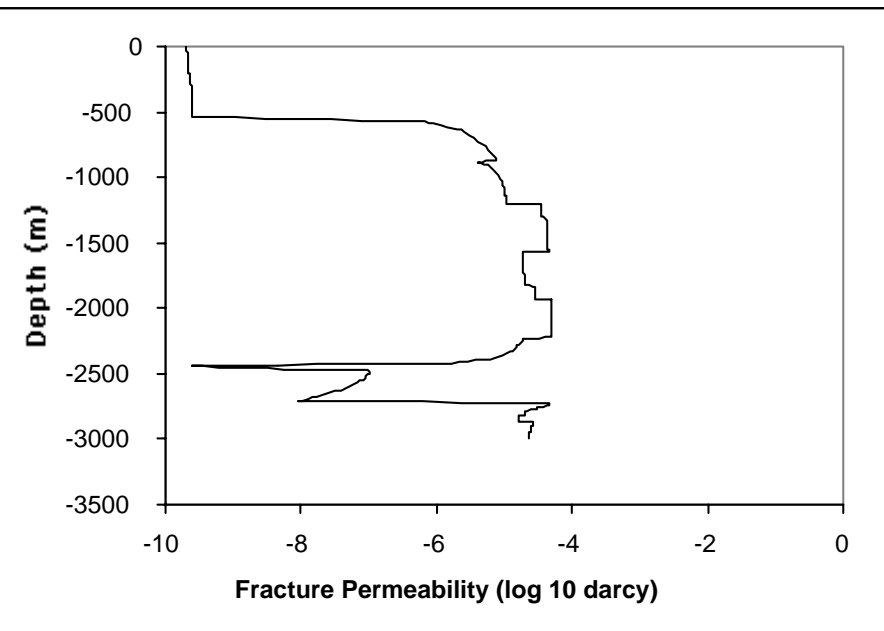

c)

Fig. V.5a,b,c Fracture permeability profile sequence illustrating the fracture front moving through the seal (between 2450 and 2700 meters). Overpressuring of oil and water phases primarily due to oil generation creates a fracture front moving upward through the seal. Once the overpressure is released, the fractures close, which in turn results in descent of the fracture front and overpressuring restarts. This cycle continues until the oil generation rate shows down, or the seal remains fractured due to tectonic effects. 
Predictive power is limited for less rigorous approaches that use statistical correlations. For example, in such methods, porosity history is often based on a formula relating it or its rate of change to mineralogy and depth of burial. However, porosity evolves due to the detailed stress, fluid composition and pressure, and thermal histories. These histories are different for every basin or part of a basin. Thus, a simple correlation of porosity with depth and lithologic type does not exist in principle. Basin RTM avoids such problems by solving the fully coupled rock deformation, fluid and mineral reaction, fluid transport and heat transfer problems. Statistical correlations give the average behavior. As "on the average" there are no interesting features such as producible pools of petroleum, such approaches can only have a limited interest.

The interplay of geological and physico-chemical information in Basin RTM is suggested in Fig. V.2. Consider one forward time step in a Basin RTM simulation. The purpose of the incremental evolution step is to advance the state of the basin from a time $t$ to a later time $t+d t$. Two distinct operations take place simultaneously during this time interval $d t$. The geological information is used to 1) fix the input/output of energy and mass at the basin boundaries and 2) impose the tectonic history (i.e., the overall basin deformation or stress) at the basin boundaries. On the other hand, the physico-chemical processes are used to determine the evolution in $d t$ of the spatial distribution of the local state. The latter describes stress, fluid properties, mineral content, rock texture, fracture characteristics and temperature. Secondary properties (permeability, rock rheological parameters, thermal conductivity and reactive surface area) are computed at each time in terms of these fundamental state variables.

\section{$1 \quad$ Input Data}

Basin RTM geological input data is divided into four categories (see Fig. V.2). The tectonic data gives the change in the lateral extent and the shape of the basement-sediment interface during $d t$. This data provides the conditions at the basin boundaries needed to calculate the change in the spatial distribution of stress and rock deformation within the basin. This latter physico-chemical calculation is carried out by a stress/deformation module that solves equations for incremental stress rock rheology and force balance (see Tuncay, Park and Ortoleva 2000a).

The next type of Basin RTM geological data affects the fluid transport, pressure and 
composition. This fluid data includes sea level changes, basin recharge conditions and the composition of fluids injected from the ocean, meteoric and basement sources. This history of boundary input data is used by the hydrologic and chemical modules to calculate the evolution of the spatial distribution of fluid pressure, fluid composition and fluid phases within the basin. These physico-chemical calculations are based on single or multi-phase flow in a porous medium and on fluid phase molecular species conservation of mass (i.e., the reaction-transport equations). The physico-chemical equations draw on internal data banks for permeability-rock texture relations, relative permeability formulae, chemical reaction rate laws and reaction and phase-equilibrium thermodynamics.

The spatial distribution of heat flux imposed at the bottom of the basin is another geological input/control. This data as well as the temperature imposed at the top of the sediment pile (i.e., climate and ocean-bottom temperature) is used to evolve the spatial distribution of temperature within the basin during the time interval $d t$. This evolution is computed using the equations of energy conservation and data for mineral and rock thermal properties (conductivities and specific heats).

The sedimentation data provides the detailed textural characteristics such as grain size, shape, mineralogy, mode and organic texture of the sediment being deposited during $d t$. This history is automatically computed by Basin RTM using interpreted well log, seismic, core and surface data. The physico-chemical laws and data are used to calculate the change of the spatial distribution of mineral and organic texture within the basin during $d t$. These physico-chemical calculations involve the rate laws for free face grain chemical kinetics, pressure solution and grain rotation or breakage, grain nucleation and the laws of kerogen chemical kinetic transformation. Also used are the laws of fracture nucleation, extension and aperture dynamics and the kinetics of cement infilling characterized via a statistical distribution of these variables (Tuncay, Park and Ortoleva 2000a,b).

\section{Numerical Solution}

We use the updated Lagrangian approach to solve the time-dependent large deformation problem for geological materials satisfying the incremental stress rheology (Bathe et al. 1975; Bathe 1996; Tuncay, Park and Ortoleva 2000a). In our numerical approach, all variables are referred to an updated configuration in each time step. The approach has two major steps. First, the incremental stress rheology equations are solved at the integration 
points of the finite elements. Second, the displacements are computed by using a global deformation solver. At each time step, iterations of these two steps are performed until the norm of the change in displacements between two consecutive iterations is less than a specified tolerance. The two-step solution technique allows the introduction of new deformation processes with only minor changes in the code. We use the conjugate gradient iterative technique with a simple diagonal preconditioner to solve for the incremental displacements. The finite element code and iterative solver are parallelized. The details of the finite element formulation is provided in Tuncay, Park and Ortoleva (2000a).

In the multi-phase module, the Galerkin-type finite element approximation is used for saturations, concentrations, and pressures. The nonlinear terms and boundary conditions are treated in a fully implicit manner. An upwinding method is developed and implemented in the multiphase module to stabilize the saturation fronts. The mass matrices are lumped to increase the stability as suggested in previous studies (Huyakorn et al. 1994; Abriola and Rathfelder 1993). The computer model accommodates a wide variety of boundary conditions. Because of the highly nonlinear behavior of the equations and the necessity for large time steps, a Newton-Raphson technique is employed to solve the nonlinear algebraic equations arising from the discretization.

The finite element grid accretes with sediment infilling. A new sediment layer is introduced when the sediment layer at the top of the basin reaches a critical thickness. In contrast, when erosion creates a top layer that is locally too thin, the finite element grid is locally reorganized to preserve numerical and topographical accuracy. This accreting, reorganizing grid that also adapts to sedimentary features as they are added is required to capture sedimentary detail and insure numerical stability and accuracy.

The interaction of the top of the sediment pile with the overlying fluids (atmosphere or sea bottom) is accounted for by the value of normal stress and the (assumed) absence of tangential shear. The no-shear lateral boundary condition allows for natural compaction at the sides of the basin. Lateral compression/extension and subsidence/upheaval are imposed at the sides and bottom. The sides and bottom are assumed to be impermeable to fluid flow.

All computational modules are packaged in an overall structure that insures all equations are satisfied at each time step. The time step dynamically changes to insure accuracy and computational efficiency. Thus time step is short when an "explosive" event is 
taking place and is long during "sleepier" epochs.

All this geological input data and physico-chemical calculations are integrated in Basin RTM over many time steps $d t$ to arrive at a prediction of the evolution history and present-day internal state of a basin or field. In this way, the physico-chemical laws are used to translate the geological input data from selected sites into a prediction of the internal state over a basin's history from its inception (or other chosen initial state) to the present.

\section{Information Theory}

Although basin models require a large number of phenomenological parameters as well as geologic boundary conditions, only a few studies focused on the utilization of observed data to constrain the model (Lerche 1991; Zhao and Lerche 1993; Maubege and Lerche 1993; Yu et al. 1995; Tuncay and Ortoleva 2002). Yu et al. (1995) used observed porosity, permeability, fluid pressure, and layer thickness data to evaluate two parameters that appear in empirical porosity and permeability expressions. However, their study lacks the assessment of uncertainty associated with the predictions.

Uncertainties in the input data needed to run a basin model lead to uncertainties in the predictions. Furthermore, formulating this input data is an extremely labor-intensive and subjective process. We suggest that basin modeling is naturally placed within the context of probability theory as follows. Let $\rho[B]$ be the probability of the boundary tectonic scenario $B$. The objective is to construct $\rho[B]$ and thereby find the most probable $B$. Once the most probable $B$ is determined, we can use it with the basin model to predict the likely location and characteristics of the reservoirs in a study area. As we have $\rho[B]$, we can also determine the uncertainties in any of the reservoir location and state parameters. With this, the basin modeling effort should focus on the development of basin data collection procedures that reduce the uncertainties implied quantitatively by the form of the dependence of $\rho$ on $B$.

As the objective is to decrease uncertainty, we wish to obtain sufficient information to limit the range in $B$ over which $\rho$ is non-negligible. Information theory (Jaynes 1957) provides a general prescription for constructing $\rho[B]$ using the information we know about the system. While the difference between the observed and synthetic data provides an error that can be minimized to calibrate a few model parameters, it cannot, given the sparseness of 
real data sets, give the most probable boundary tectonic scenario, nor can such an error minimization procedure yield a self-consistent assessment of risk.

A probabilistic basin modeling approach can also provide a natural platform for the integration of expertise. These expertise constraints include limiting the spatial and temporal scale of phenomena (e.g. maximum known rate of overall basin deformation, basement heat flux, etc.). In the following we show that this can conveniently be done via the minimum relative entropy approach. As a model becomes more comprehensive or the number of expertise constraints are increased, less data is needed. If the above data/modeling integration can be automated, then a basin model in effect becomes the centerpiece of a database mining algorithm since differential equations of physics and chemistry are simply algorithms for processing information. In this sense, this procedure is the essence of a quantitative geoinformatics methodology.

A central challenge of basin modeling is to construct the present-day internal configuration and chronology of the subsurface from data of a range of types and quality, sparsely distributed across a study area. As the available data is typically indirect and fraught with uncertainty, an objective methodology is needed that yields the most probable chronology and present-day configuration as well as an estimate of the associated uncertainty. Further, there is often a great quantity of data that is too time-consuming to fully integrate by classical methods and methods of analysis are often subject to individual bias. We now outline the MAI (Model-Automated Informatics) approach to the challenges of petroleum $\mathrm{E} \& \mathrm{P}$ based on an information theory (probabilistic) integration of basin modeling and large databases.

Basin data must be integrated with modeling to compensate for the incompleteness of both. Furthermore, the integration must be automated, i.e., seismic, well log, core and other data must, to the extent possible, be used as direct input to the model so as to reduce labor-intensive tasks and to eliminate bias in the interpretation of the geological record. On the other hand, the automation should somehow integrate our geological experience/expertise in a natural way so as to minimize the extent of the computations.

There are two main categories of factors that an automated procedure must determine to run a model. The first is the least well-known parameters in the phenomenological expressions and, secondly, the scenario of factors influencing the basin at 
its boundaries (uplift/subsidence and compression/extension/wrenching; basement heat flux, climate, and sediment/erosion). The latter factors change across the basin's boundaries and over the history of the basin. Below we outline a new approach, presenting a derivation of an equation for the most probable history of the spatial distribution of the least wellconstrained boundary factors. Having delineated these factors, one may use them with a basin model to estimate the time-course of the internal state of a basin from the inception of a basin to the present. This approach has been demonstrated for an engineering problem (Tuncay and Ortoleva 2002).

Let $B$ represent the time course of a set of influences acting on the basin boundaries. Thus, $B$ represents the histories of compression/extension and upheaval/subsidence or of other factors (basement heat and fluid flux, sea level, sediment input, erosion) acting at each point on the basin's top, side and bottom. Let $\rho$ be the probability of a given scenario $B$ of these influences. For example, if $B$ represents the basement heat flux at all points on the basin bottom for all times of the basin's history (inception to present), then $\rho$ depends on the infinity of these heat fluxes (all points on the bottom for all times). Let $S$ indicate an integration of the infinity of such variables, i.e. a functional integral; then normalization of $\rho$ implies $S_{B} \rho=1$. Information theory (Jaynes 1957) in the present context is based on the entropy $S$ defined via

$S=-S_{B} \rho \ln \rho$.

$S$ is taken to be a measure of the uncertainty we have in the state of a system; thus as the number of possible states of the system increases so does the entropy. The probability $\rho$ is determined to be that functional of $B$ which maximizes $S$ subject to (V.1) and information we may have about the system (e.g. seismic, well log, fluid pressure, core analysis, etc).

Let $O^{(k)}$ be the $k$-th set of the aforementioned data we have on the basin $\left(k=1,2, \ldots, N_{\text {error }}\right)$. Thus $O^{(1)}$ could be a seismic survey on one area, $O^{(2)}$ is a survey on another, $O^{(3)}$ is a suite of $\operatorname{logs}$ at various locations, etc. Similarly, let $\Omega^{(k)}$ be the synthetic seismic or other data as constructed using a basin simulator. With this, we construct the $k$-th error $E^{(k)}$ defined such that 
$E^{(k)}=\sum_{j=1}^{N^{(k)}}\left(\Omega_{j}^{(k)}[B]-O_{j}^{(k)}\right)^{2}$

As a basin simulation depends on $B$, then so does the synthetic data $\Omega^{(k)}$ constructed from it. In (V.3), $N^{(k)}$ is the number of data values of type $k, O_{j}^{(k)}$ is the $j$-th value of type $k$ $j=1,2, \ldots, N^{(k)}$ and $\Omega_{j}^{(k)}$ is the synthetic value of $O_{j}^{(k)}$. With this, we impose the conditions

$$
S_{B} \rho E^{(k)}=E^{(k)^{*}}
$$

where $E^{(k)^{*}}$ is an estimated value of $E^{(k)}$ obtained from our general knowledge of the accuracy of the available data, numerical accuracy of the basin simulator and the formulas used to construct $\Omega^{(k)}$ from it.

The spatial sparseness of the available data does not allow us to determine the spatial dependence of $B$ on short length scales. Furthermore, practical basin simulation does not allow for very fine grid spacing. Thus, it is not feasible to seek a very fine spatial scale resolution of the $B$-parameters. A similar consideration holds for the time dependence of the $B$-parameters. To constrain the scale at which we wish to delineate the space-time variations in the state of the system, we impose the conditions

$$
\mathrm{S}_{B} \rho \int_{0}^{t} d t \frac{1}{A} \int_{\text {boundary }} d^{2} r \frac{1}{2}\left|(\underline{\nabla}-\underline{n}(\underline{n} \cdot \underline{\nabla})) B_{\alpha}\right|^{2}=\chi_{\alpha}
$$

where $t_{\text {present }}$ is the age of the basin ( $t=0$ being the time at basin inception), and $\underline{n}$ is a unit normal to the basin's boundary pointing outward; $\underline{n}, \underline{\nabla}$ terms imply a tangential gradient. Similarly

$$
\mathrm{S}_{B} \rho \int_{0}^{{ }^{t} \text { present }} d t \frac{1}{A} \int_{\text {boundary }} d^{2} r \frac{1}{2}\left(\frac{\partial B_{\alpha}}{\partial t}\right)^{2}=\theta_{\alpha}
$$

In the above, $B_{\alpha}$ is the $\alpha$-th of the $N_{b}$ boundary factors $\left(B=\left\{B_{1}, B_{2}, \ldots, B_{N_{b}}\right\}\right.$ and $\chi_{\alpha}$ and $\theta_{\alpha}$ are estimates that constrain the spatial and time derivatives of $B_{\alpha}$ while $A$ is the (timedependent) surface area of the basin.

In the spirit of information theory (Jaynes 1957), we maximize $S$ subject to the constraints (V.1, 4, 5 and 6) to obtain $\rho$ : we find 


$$
\begin{aligned}
\ln \rho= & -\ln \mathrm{Q}-\sum_{k=1}^{N_{\text {gror }}} \beta_{k} E^{(k)} \\
& -\sum_{\alpha=1}^{N_{b}} \lambda_{\alpha} \int_{0}^{t_{\text {present }}} d t \frac{1}{A} \int_{\text {boundary }} d^{2} r\left|(\underline{\nabla}-\underline{n}(\underline{n} \cdot \underline{\nabla})) B_{\alpha}\right|^{2} \\
& -\sum_{\alpha=1}^{N_{b}} \omega_{\alpha} \int_{0}^{t_{\text {present }}} d t \frac{1}{A_{\text {boundary }}} \int_{d^{2}} d^{2} r\left(\frac{\partial B_{\alpha}}{\partial t}\right)^{2} .
\end{aligned}
$$

This completes the formal construction of $\rho$ (once the normalization constant $\mathrm{Q}$ is evaluated via (V.1) and the Lagrange multipliers ( $\beta, \lambda . \omega$ - parameters) are fixed via (V.4 5 and 6)).

The most probable space-time dependence of the $B_{\alpha}$ are determined to be those which maximize $\rho$. We find, upon setting the functional derivatives of (V.7) to zero,

$$
\frac{\delta \ln \rho}{\delta B_{\alpha}}=0 \quad\left(\alpha=1,2, \ldots, N_{b}\right)
$$

To find $B$ we solve this set of functional differential equations.

Our approach can be more explicitly illustrated for the most probable history of the basement heat flux. Let $B(x, y, t)$ be the vertical heat flux into the bottom of a basin at map view position $x, y$ at time $t$. For the simple case of a single type of error $E$ we have $\beta \frac{\delta E}{\delta B(x, y, t)}-\frac{\chi}{A}\left\lfloor\frac{\partial^{2} B}{\partial x^{2}}+\frac{\partial^{2} B}{\partial y^{2}}\right\rfloor-\frac{\theta}{A} \frac{\partial^{2} B}{\partial t^{2}}=0$

for area $A$ of the basin bottom taken to be constant for simplicity of illustration here. This equation has the character of a space-time diffusional dynamic interacting with the nonlinear $\delta E / \delta B$ term that is a functional derivative of $E$ with respect to $B(x, y, t)$. The $\delta E / \delta B$ term are constructed using a basin simulator.

In the approach presented above, two conditions (V.5 and V.6) are used to constrain the spatial and temporal resolution of the $B$-parameters. The use of prior information to regularize the inverse problems is a common practice. This can be achieved through an additional error measure in the form of

$$
E_{\alpha}^{B}=\sum_{j=1}^{M}\left(B_{\alpha}^{j}-B_{\alpha}^{j p}\right)^{2}
$$


where $M$ is the number of points used to discretize $B$ and $B_{\alpha}^{j p}$ is the prior information which is usually taken as the initial guess. The use of prior information is convenient. However, it may also be misleading if it masks the observed data. An alternative approach to account for prior information is through the use of the minimum relative entropy principle (Kullback 1959; Kapur 1988; Woodbury and Ulrych 1996). The minimum relative entropy principle suggests that the probability $\rho$ is constructed by the minimization of the functional $H$ : $H=S_{B} \rho \ln \frac{\rho}{q}$

with respect to $\rho$. In equation (V.11) $q$ is the a priori probability distribution. The use of a quadratic error measure (such as V.10) for the set of uncertain parameters corresponds to the assumption of a gaussian distribution for the prior information. The minimum relative entropy principle allows one to tailor the a priori probability to the degree of uncertainty once has accumulated through the experience/expertise.

An approximation to $\rho$ can be obtained by expanding (V.7) around the most probable $B$ $\left.\ln \rho \approx \ln \rho\right|_{\underline{B}^{m}}+\left.\sum_{\alpha=1}^{N_{b}} \sum_{i=1}^{n} \frac{\partial \ln \rho}{\partial B_{\alpha}^{i}}\right|_{\underline{B}^{m}} \Delta B_{\alpha}^{i}+\left.\sum_{\alpha=1}^{N_{b}} \sum_{i, j=1}^{n} \frac{1}{2} \frac{\partial^{2} \ln \rho}{\partial B_{\alpha}^{i} \partial B_{\alpha}^{j}}\right|_{\underline{B}^{m}} \Delta B_{\alpha}^{i} \Delta B_{\alpha}^{j}+\cdots$

At the most probable scenario $\left(\underline{B}^{m}\right)$ the second term vanishes. This approximate probability distribution accounts for all the available information, i.e., geologic data and associated error, a priori information, regularization constraints for temporal and spatial distributions. The matrix of the quadratic term is constructed in the course of carrying out a large number of basin simulations to solve (V.8) numerically. Equation (V.12) is used to assess the uncertainty associated with the $B$ parameters. 


\section{Develop Seismic Inversion Code Using Reciprocity Principle (Subtask 4.5)}

\section{A Introduction}

Seismic waveform inversion can be defined as an iterative procedure for obtaining accurate parameters of the Earth from prestack seismic reflection data. Early studies include Lailly (1983) and Tarantola (1984) who showed the adjoint state method for constructing the steepest descent direction for the inversion of the acoustic problem without computing the partial derivatives explicitly. This method finds the gradient direction by cross-correlating forward propagated wavefields from a seismic source with backward propagated wavefields from the data residuals. As each iterative loop of the inversion requires only several forward wave propagation simulations for each seismic source, it made seismic waveform inversion feasible in 1980s. Mora (1987) applied this method to elastic problems in time domain and Pratt et al. (1998) to acoustic problems in frequency domain. Despite its low demand of computations, this inversion scheme suffered from recovering background velocity information (Gauthier et al. 1986; Mora 1987; Crase et al. 1990; Hicks and Pratt 2001).

Tarantola (1984) also presented the algorithm called 'total inversion' (Tarantola and Valette 1982), which was impossible to be implemented at the time it was presented because of the incapability of computers. In recent years, however, it has become feasible. Pratt et al. (1998) used 'virtual source' terms to make partial derivative seismic wavefields which had been used for electromagnetic problem (Rodi 1976). The partial derivative wavefields are obtained from new wave propagation simulations driven by the virtual sources at the location of model parameters, which means that the virtual source simulations are needed as many as the number of model parameters.

Shin et al. (2001) presented an efficient way of calculating partial derivative wavefields using the reciprocity relation between the virtual sources and the receivers. The reciprocity theorem is proven in Aki and Richards (1980) for an elastic anisotropic continuous medium. This theorem allows the source-receiver locations to be interchanged. The recorded seismograms are identical if the sources and receivers are located inside the model or on its boundary (Eisner and Clayton 2001). 
Although developments in computer technology have been impressive, it has been impractical to make use of the Newton method for high-resolution seismic inversions. To avoid extremely expensive computation of the Hessian matrix, Hicks and Pratt (2001) proposed a two-step inversion procedure. The adjoint state method is used for finding reflectors, and then the Newton method is exploited for background velocities with a much smaller number of parameters. Shin et al. (2001) took advantage of diagonally dominant property of the 'approximate Hessian' matrix (Pratt et al. 1998). Diagonal elements of the approximate Hessian were used as a preconditioner for an interative inversion. In all of those studies, the finite element method was used to solve the forward problems in frequency domain.

The most expensive computation in seismic waveform inversions is the evaluation of partial derivative wavefields. Explicit calculation of them requires huge amounts of memory and computation, far more than resources that are available at a typical scientific workstation. However, these resources can be most cost-effectively provided nowadays by scalable massively parallel computers which are usually programmed by Message Passing Interface (MPI).

In this work, we compute the Jacobian and the approximate Hessian matrix explicitly based on time domain staggered-grid finite difference modeling scheme (Levander 1988; Graves 1996). The wavefields propagated from the sources are saved evenly at each processor, which are the virtual sources. Then, after each simulation of the reciprocal wavefields from one of the receivers, the partial derivative wavefields at the receiver are calculated from the summations of convoluted wavefields and the approximate Hessian matrix is updated.

This chapter is organized as follows: We briefly recap the inverse problem and show how to compute partial derivative wavefields. We also validate the seismic reciprocity and convolution theorem. We then present more detailed inversion scheme of this work. After reviewing how the Gauss-Newton method can improve the gradient directions, and then their inverted results are presented. We conclude by showing several numerical examples illustrating the application of the seismic waveform inversion. 


\section{B Inverse problem}

Seismic waveform inversion can be stated as the problem of finding accurate parameters of the Earth from seismic reflection data. In order to infer a set of model parameters which represent the Earth, the inverse problem seeks to minimize the residuals between the model response obtained by forward modeling procedures and the observed seismic reflection data.

In general, the seismic responses $\mathbf{d}$ of the Earth represented by model parameters $\mathbf{m}$ would be recorded at receivers. This relationship can be expressed with the nonlinear functional $\mathbf{F}$ :

$\mathbf{d}=\mathbf{F}(\mathbf{m})$,

The residual error is defined as the difference between the model responses and the observed data:

$\Delta \mathbf{d}=\mathbf{F}(\mathbf{m})-\mathbf{F}\left(\mathbf{m}^{0}\right)$,

where $\mathbf{m}^{0}$ is the a priori model. We now introduce the least-squares problem:

$S_{d}(\mathbf{m})=\frac{1}{2} \Delta \mathbf{d}^{t} \Delta \mathbf{d}$,

where $S_{d}$ means the data misfit function and the factor $1 / 2$ allows subsequent simplifications and the superscript $t$ represents the matrix transpose. The inverse problem becomes the minimization of $S_{d}$. Thus, our purpose is to find a model $\mathbf{m}^{*}$ such that $S_{d}\left(\mathbf{m}^{*}\right) \cong 0$.

Unlike for linear problems, for nonlinear problems such as seismic waveform inversion, there is no guarantee that can we find a solution from equation (VI.3) explicitly.

\section{Gradient method}

The gradient method is to minimize $S_{d}$ by updating model parameters in the opposite direction of the gradient of $S_{d}(\mathbf{m})$ iteratively:

$\mathbf{m}^{n+1}=\mathbf{m}^{n}-\alpha \nabla S_{d}$,

where the superscripts represent the iteration number and $\alpha$ is the step length. The gradient direction can be obtained by taking partial derivatives of equation (VI.3) with respect to model parameters $\mathbf{m}$ :

$\nabla S_{d}=\left(\frac{\partial \mathbf{F}_{i}(\mathbf{m})}{\partial m_{j}}\right)^{t} \Delta \mathbf{d}=\mathbf{J}^{t} \Delta \mathbf{d},(i=1, \ldots, N ; j=1, \ldots, M)$, 
where $\mathbf{J}^{t}$ is the transpose of Jacobian matrix, the subscripts $i$ and $j$ indicate the receiver location and model parameter, respectively, and $N$ and $M$ are the numbers of receivers and model parameters, respectively.

\section{Adjoint state method}

Explicit computation of the Jacobian matrix $\mathbf{J}$ for a single shot requires $M+n s h o t$ forward simulations by using the virtual source or $2 \times N+n s h o t$ by using the reciprocity theorem. In the following sections, we will examine the explicit computation of the Jacobian. However, to obtain the gradient of the data misfit function, it is not necessary to compute the Jacobian explicitly. The adjoint state method requires only several forward computations to generate the gradient (Tarantola 1984; Mora 1987, Crase et al. 1990, Pratt et al. 1998).

Formal derivations for the adjoint state method are given by Tarantola (1984) and Mora (1987). We restate the computational steps which must be required in the gradient calculation and refer to those references for more details. For the seismic inverse problem, the following steps are required to determine the gradient direction with respect to model parameters:

(1) Solve the elastic wave equation, and sample the wave fields at receiver locations and at the nodes of model parameters;

(2) Compute the weighted residual errors between sampled seismogram from step (1) and the observed data;

(3) Back propagate the weighted residuals, i.e., solve the elastic wave equation with the time reversed weighted residual sources located at receiver locations.

(4) Simultaneously, compute the gradient for the model parameters given by

$$
\delta \hat{\rho}=-\sum_{\text {Shot }} \int d t\left(\dot{\vec{u}}_{x} \dot{\bar{u}}_{x}+\dot{\bar{u}}_{z} \dot{\bar{u}}_{z}\right) \text {, }
$$

$\delta \hat{\lambda}=-\sum_{\text {Shot }} \int d t\left(\vec{u}_{x, x}+\vec{u}_{z, z}\right)\left(\bar{u}_{x, x}+\bar{u}_{z, z}\right)$,

$\delta \hat{\mu}=-\sum_{\text {Shot }} \int d t 2\left(\vec{u}_{x, x} \bar{u}_{x, x}+\vec{u}_{z, z} \bar{u}_{z, z}\right)_{j}+\left(\vec{u}_{x, z}+\vec{u}_{z, x}\right)\left(\bar{u}_{z, x}+\bar{u}_{x, z}\right)$

where $\vec{u}_{x}$ and $\vec{u}_{z}$ are displacement of the forward wavefields from step (1), and $\bar{u}_{x}$ and $\bar{u}_{z}$ are displacement of the back propagated wavefields from step (3). 


\section{E Newton method \& Gauss-Newton method}

The Newton method is an effective and robust technique for numerical optimization of nonlinear problems and provides faster convergence rates than the gradient method. At the final stages of the gradient method and the adjoint state method, we may have nearly zero values of the residual and the gradient of $S_{d}$. However, it is not guaranteed to converge to a minimal solution of $S_{d}$. Recall that a technique for finding a point to minimize a continuous and differentiable function is to take the derivative, find a value makes the derivative zero, and check the second derivative is positive in order that the point can be a minimum. The Hessian matrix is the analog of the second derivative and it is required that the Hessian matrix should be positive definite. Equation (VI.4) can be linearized by a second order Taylor expansion:

$$
\begin{aligned}
S_{d}\left(\mathrm{~m}^{*}\right) & =S_{d}(\mathrm{~m}+\Delta \mathrm{m}) \\
& \cong S_{d}(\mathrm{~m})+\Delta \mathrm{m}^{t} \nabla S_{d} \Delta \mathrm{d}+\frac{1}{2} \Delta \mathrm{m}^{t} \nabla^{2} S_{d} \Delta \mathrm{m}+O\left(\Delta \mathrm{m}^{3}\right) \\
& =S_{d}(\mathrm{~m})+\Delta \mathrm{m}^{t} \mathrm{~J}^{t} \Delta \mathrm{d}+\frac{1}{2} \Delta \mathrm{m}^{t} \mathrm{H} \Delta \mathrm{m}+O\left(\Delta \mathrm{m}^{3}\right),
\end{aligned}
$$

where $\mathbf{H}$ is the Hessian matrix. In order to seek a vector $\Delta \mathbf{m}$ such that the gradient of $S_{d}$ $\left(\mathbf{m}^{*}\right)$ is zero, take the gradient with respect to $\Delta \mathbf{m}$, then we get

$\nabla S_{d}(\mathbf{m} *)=0 \cong 0+\mathbf{J}^{t} \Delta \mathbf{d}+\mathbf{H} \Delta \mathbf{m}$,

or

$\mathbf{H} \Delta \mathbf{m}=-\mathbf{J}^{t} \Delta \mathbf{d}$.

Assuming that the Hessian matrix is positive definite, we can solve for $\Delta \mathbf{m}$ and the Newton method is given by

$\mathbf{m}^{n+1}=\mathbf{m}^{n}-\mathbf{H}^{-1} \mathbf{J}^{t} \Delta \mathbf{d}$

Each element of the Hessian matrix can be expressed in differential forms:

$$
\mathbf{H}=\nabla^{2} S_{d}(\mathbf{m})=\frac{\partial}{\partial m_{p}}\left(\mathbf{J}^{t} \Delta \mathbf{d}\right)=\mathbf{J}^{t} \mathbf{J}+\frac{\partial}{\partial m_{p}}\left(\mathbf{J}^{t}\right) \Delta \mathbf{d} .
$$

Because the second term of equation (VI.11) is usually small and negligible (Tarantola, 1987), we obtain the Gauss-Newton formula

$\mathbf{m}^{n+1}=\mathbf{m}^{n}-\left(\mathbf{J}^{t} \mathbf{J}\right)^{-1} \mathbf{J}^{t} \Delta \mathbf{d}$,

where $\mathbf{J}^{\mathbf{t}} \mathbf{J}$ is the approximate Hessian matrix. 
In the application of the Gauss-Newton method to geophysical inversion, regularization methods are particularly useful for stabilizing the system and incorporating $a$ priori information to the problem (Tarantola 1987). The regularized misfit function $S$ can be defined as,

$S(\mathbf{m})=S_{d}(\mathbf{m})+\lambda S_{m}(\mathbf{m})$,

where $S_{m}$ is the model objective function that contains a priori information of the model and $\lambda$ is a scalar value that globally controls the relative importance of the model objective function $S_{m}$. The model objective function can be written as combinations of discrete linear operator $\mathbf{L}$ :

$S_{m}(\mathbf{m})=\frac{1}{2}(\mathbf{L} \Delta \mathbf{m})^{t}(\mathbf{L} \Delta \mathbf{m})$.

Then the regularized Gauss-Newton formula can be written as,

$\mathbf{m}^{n+1}=\mathbf{m}^{n}-\alpha^{n}\left(\mathbf{J}^{t} \mathbf{J}+\lambda \mathbf{L}^{t} \mathbf{L}\right)^{-1} \mathbf{J}^{t} \Delta \mathbf{d}$,

where $\alpha$ is a step length that can be regarded as a scaling factor for balancing between data and model dimensions.

If $\mathbf{L}=\mathbf{I}$ (the identity matrix), equation (VI.15) yields the damped least-squares method (Levenberg 1944; Marquardt 1963):

$$
\mathbf{m}^{n+1}=\mathbf{m}^{n}-\alpha^{n}\left(\mathbf{J}^{t} \mathbf{J}+\lambda \mathbf{I}\right)^{-1} \mathbf{J}^{t} \Delta \mathbf{d} .
$$

A choice of $\lambda$ between 0 and infinity produces a compromise direction. As $\lambda$ goes to infinity, the direction approaches steepest descent, which means that the Gauss-Newton method gets close to the Gradient method.

If $\mathbf{L}$ is a discrete spatial differential operator, the model objective function controls the roughness of spatial variations among the model parameters. Sasaki (1989) used discrete 2-D Laplacian operator:

$$
L_{i} \mathbf{\Delta} \mathbf{m}=\left(\Delta m_{i}\right)^{E}+\left(\Delta m_{i}\right)^{W}-4\left(\Delta m_{i}\right)+\left(\Delta m_{i}\right)^{N}+\left(\Delta m_{i}\right)^{S},
$$

where the superscripts $E, W, N$, and $S$ refer to the four neighbors of the $i$ th model parameter.

The simplest way of choosing the step length is to take it as a constant through all the iterations, which can be obtained by trial and error. In this work, an optimal value of the step length is determined by a linearized approach. Equation (VI.15) can be written as $\mathbf{m}^{n+1}=\mathbf{m}^{n}-\alpha^{n} \mathbf{g}^{n}$, 
where

$$
\mathbf{g}^{n}=\left(\mathbf{J}^{t} \mathbf{J}+\lambda \mathbf{L}^{t} \mathbf{L}\right)^{-1} \mathbf{J}^{t}\left[\mathbf{d}^{n}-\mathbf{d}^{0}\right]
$$

In order to estimate an adequate value of $\alpha$, a new error functional $S^{\prime}$ is given by

$$
S^{\prime}\left(\mathbf{m}^{n}-\alpha^{n} \mathbf{g}^{n}\right)=\frac{1}{2}\left[\mathbf{F}\left(\mathbf{m}^{n}-\alpha^{n} \mathbf{g}^{n}\right)-\mathbf{d}^{0}\right]^{t}\left[\mathbf{F}\left(\mathbf{m}^{n}-\alpha^{n} \mathbf{g}^{n}\right)-\mathbf{d}^{0}\right] .
$$

The Taylor series expansion yields

$$
\mathbf{F}\left(\mathbf{m}^{n}-\alpha^{n} \mathbf{g}^{n}\right) \cong \mathbf{F}\left(\mathbf{m}^{n}\right)-\alpha^{n} \mathbf{J}^{t} \mathbf{g}^{n}
$$

Substituting equation (VI.21) into equation (VI.20) yields

$$
S^{\prime}\left(\mathbf{m}^{n}-\alpha^{n} \mathbf{g}^{n}\right) \cong S^{\prime}\left(\mathbf{m}^{n}\right)-\left(\alpha^{n} \mathbf{J}^{t} \mathbf{g}^{n}\right)^{t}\left[\mathbf{F}\left(\mathbf{m}^{n}\right)-\mathbf{d}^{0}\right]+\frac{1}{2}\left(\alpha^{n}\right)^{2}\left(\mathbf{J}^{t} \mathbf{g}^{n}\right)^{t}\left(\mathbf{J}^{t} \mathbf{g}^{n}\right),
$$

and solving for the derivative of $S^{\prime}$ with respect to $\alpha^{n}$ yields

$$
\frac{\partial S^{\prime}\left(\mathbf{m}^{n}-\alpha^{n} \mathbf{g}^{n}\right)}{\partial \alpha^{n}} \cong-\left(\mathbf{J}^{t} \mathbf{g}^{n}\right)^{t}\left[\mathbf{F}\left(\mathbf{m}^{n}\right)-\mathbf{d}^{0}\right]+\alpha^{n}\left(\mathbf{J}^{t} \mathbf{g}^{n}\right)^{t}\left(\mathbf{J}^{t} \mathbf{g}^{n}\right) \text {. }
$$

Then, setting the derivative to zero yields the optimal value for $\alpha^{n}$,

$$
\alpha^{n} \cong \frac{\left(\mathbf{J}^{t} \mathbf{g}^{n}\right)^{t}\left[\mathbf{F}\left(\mathbf{m}^{n}\right)-\mathbf{d}^{0}\right]}{\left(\mathbf{J}^{t} \mathbf{g}^{n}\right)^{t}\left(\mathbf{J}^{t} \mathbf{g}^{n}\right)}
$$

\section{F Elastic wave equations}

In 2-D Cartesian coordinates, for an isotropic, linearly elastic medium, the equations of motion can be written as:

$$
\begin{aligned}
& \rho \ddot{u}_{i}=\tau_{i j, j}+f_{i}, \\
& \tau_{i j}=2 \mu e_{i j}+\delta_{i j} \lambda e_{k k}+g_{i j},
\end{aligned}
$$

where

$u$ : displacement vector;

$\tau$ : stress tensor;

$e_{i j}$ : strain tensor defined by $\left(u_{i, j}+u_{j, i}\right) / 2$;

$f_{i}$ and $g_{i j}$ : body force source and traction source;

$\rho$ : density;

$\lambda$ and $\mu$ : Lamé coefficients;

$\delta_{i j}$ : Kronecker delta $\left(\delta_{i j}=0\right.$ for $i \neq j$ and $\delta_{i j}=1$ for $\left.i=j\right)$;

$\because$ : temporal derivative, $\partial^{2} / \partial t^{2}$; 
${ }_{j}:$ spatial derivative, $\partial / \partial x$ or $\partial / \partial y$;

and the summation convention is used:

$$
e_{k k}=e_{i i}+e_{j j}
$$

These equations can be expressed as a set of first-order hyperbolic equations:

$$
\begin{aligned}
& \rho \dot{v}_{i}=\tau_{i j, j}+F_{i}, \\
& \dot{\tau}_{i j}=\mu\left(v_{i, j}+v_{j, i}\right)+\lambda \delta_{i j} v_{k, k}+G_{i j},
\end{aligned}
$$

where $v$ is velocity, and $F_{i}$ and $G_{i j}$ represent seismic sources, respectively.

\section{G Partial derivative seismic wavefields}

The partial derivative field with respect to model parameters is required to perform seismic waveform inversion. In our formulation, the model parameters are the density, $\rho$, and the two Lamé parameters, $\lambda$ and $\mu$. In the case of the density as the model parameter, equation (VI.26) is differentiated with respect to a $\rho_{p}$ :

$$
\begin{aligned}
& \rho \frac{\partial \dot{v}_{i}}{\partial \rho_{p}}=\frac{\partial \tau_{i j, j}}{\partial \rho_{p}}-\frac{\partial \rho}{\partial \rho_{p}} \dot{v}_{i}, \\
& \frac{\partial \dot{\tau}_{i j}}{\partial \rho_{p}}=\mu\left(\frac{\partial v_{i, j}}{\partial \rho_{p}}+\frac{\partial v_{j, i}}{\partial \rho_{p}}\right)+\lambda \delta_{i j} \frac{\partial v_{k, k}}{\partial \rho_{p}},
\end{aligned}
$$

where $-\left(\partial \rho / \partial \rho_{p}\right) \dot{v}_{i}$ is a virtual source for the partial derivative seismogram $\partial v_{i} / \partial \rho_{p}$, which is activated at the location of a particular model parameter $\rho_{p}$. In the case of two Lamé parameters, partial derivative wavefields can be obtained from the following equations, respectively,

$$
\begin{aligned}
& \rho \frac{\partial \dot{v}_{i}}{\partial \mu_{p}}=\frac{\partial \tau_{i j, j}}{\partial \mu_{p}}, \\
& \frac{\partial \dot{\tau}_{i j}}{\partial \mu_{p}}=\mu\left(\frac{\partial v_{i, j}}{\partial \mu_{p}}+\frac{\partial v_{j, i}}{\partial \mu_{p}}\right)+\lambda \delta_{i j} \frac{\partial v_{k, k}}{\partial \mu_{p}}+\frac{\partial \mu}{\partial \mu_{p}}\left(v_{i, j}+v_{j, i}\right),
\end{aligned}
$$

and

$$
\begin{aligned}
& \rho \frac{\partial \dot{v}_{i}}{\partial \lambda_{p}}=\frac{\partial \tau_{i j, j}}{\partial \lambda_{p}}, \\
& \frac{\partial \dot{\tau}_{i j}}{\partial \lambda_{p}}=\mu\left(\frac{\partial v_{i, j}}{\partial \lambda_{p}}+\frac{\partial v_{j, i}}{\partial \lambda_{p}}\right)+\lambda \delta_{i j} \frac{\partial v_{k, k}}{\partial \lambda_{p}}+\frac{\partial \lambda}{\partial \lambda_{p}} \delta_{i j} v_{k, k},
\end{aligned}
$$


where $\left(\partial \mu / \partial \mu_{p}\right)\left(v_{i, j}+v_{j, i}\right)$ and $\left(\partial \lambda / \partial \lambda_{p}\right) \delta_{i j} v_{k, k}$ are virtual sources for the partial derivative seismograms $\partial v_{i} / \partial \mu_{p}$ and $\partial v_{i} / \partial \lambda_{p}$, respectively. This approach follows from Rodi (1976) and Pratt et al. (1998) who formulated the problem in frequency domain. By analogy with equation (VI.26), the wavefield solutions of equations (VI.27)-(VI.29) are partial derivative wavefields due to virtual seismic sources at the perturbed model parameter location. The virtual sources in equations (VI.28) and (VI.29) are constructed using solution of equation (VI.26).

\section{H Virtual source, Seismic reciprocity \& convolution}

In this section, we consider only the case of equation (VI.27). However, other cases are also implemented. In order to solve equations (VI.26) and (VI.27), a 2-D elastic velocity-stress staggered-grid finite difference method (Levander 1988; Graves 1996) is used, which includes the implementation of the absorbing boundary condition of Berenger (1994) at the bottom and two vertical boundaries. A combination of the stress-image and the adjusted finite difference method (Sheen et al. 2004) is used as the free surface boundary condition. The finite difference formulation has the properties of fourth order accuracy in space and second order accuracy in time domain.

The synthetic seismograms generated from a simple model of $600 \mathrm{~m} x$-extent and $320 \mathrm{~m} z$-extent are used to illustrate our algorithm. The first time derivative of the Gaussian function with a frequency of $100 \mathrm{~Hz}$ is used as the source time function. The grid spacing is $0.4 \mathrm{~m}$ and the time step is $80 \mu \mathrm{s}$.

We consider a homogeneous model with a perturbed density block whose size is $8 \mathrm{~m}$ $\times 8 \mathrm{~m}$ and the source and the receiver are located at the free surface (Fig. VI.1). To test the accuracy of equation (VI.27), first, we simulate the seismic wave propagation without the perturbed block and record seismograms at the receiver and the block. Next simulation is performed with the block and a seismogram is recorded at the receiver. From the difference between seismograms at the receiver, we obtain the partial derivative seismogram of the second order accuracy. We, then, solve equation (VI.27) with the virtual source (VS) recorded at the block. Fig. VI.2 shows the vertical (VC) and horizontal component (HC) of the partial derivative seismograms obtained by the straight forward difference approach and the solution of equation (VI.27). As seen in the figure, the partial derivative seismograms are identical. 
Fig. VI.3 illustrates the reciprocity theorem. Configuration (i) can be decomposed into (ii) and (iii). By the reciprocity theorem, those configurations can be replicated by (iv) and (v). The relations between configurations (iii) and (v) of Fig. VI.3a and between (ii) and (iv) of Fig. VI.3b show that the locations of source and receiver should be reciprocal as well as the orientations (Arntsen and Carcione 2000). Thus, for example, the reciprocal simulation of the horizontal motion from the source can be generated by summing horizontal and vertical responses from a horizontal source.

The reciprocity relation is investigated with a VS recorded inside the medium. Fig. VI.4 shows a two layer model in which a pair of source/receiver locations is marked. To validate the reciprocity between the free surface and inside the model, the amplitude of the $\mathrm{HC}$ of a source on the free surface should be twice because of the characteristic of the free boundary. Thus, in our experiments, all HCs of a surface source are doubled for the reciprocity relation.

In Fig. VI.5, the first time derivative of the Gaussian function source wavelet is assigned to the HC (Figs. VI.5a and VI.5b) and the VC (Figs. VI.5c and VI.5d), respectively. The receiver orientations of Figs. VI.5a and VI.5c are horizontal and those of Figs. VI.5b and VI.5d vertical, respectively. The discrepancies of Fig. VI.5b and VI.5c show that the reciprocity relation is only valid if both of the reciprocal location and the reciprocal orientation match (see Fig. VI.3).

We use a recorded seismogram at the VS to simulate partial derivative wavefields. In order to verify the reciprocal orientation, we run four simulations with sources: (1) HC with horizontal VS, (2) HC with vertical VS, (3) VC with horizontal VS, and (4) VC with vertical VS. The summation of HC of (1) and VC of (2) represents the horizontal response of the VS whereas the summation of $\mathrm{HC}$ of (3) and $\mathrm{VC}$ of (4) represents the vertical response of the VS (Fig. VI.6).

In frequency domain, this additional forward calculation is straight forward because the convolution of a virtual source function and the impulse response can generate the reciprocal partial derivative wavefield with ease. In time domain, although the convolution is not as trivial as in frequency domain, we follow a similar approach. For instance, first, we simulate the reciprocal wavefield with a source wavelet, such as the first time derivative of the Gaussian function, which has only a horizontal component. After the simulation, a 
horizontal partial derivative wavefield is obtained by summing horizontal and vertical responses which are convolved with virtual sources of horizontal and vertical component, respectively. These reciprocal seismograms are the same as partial derivatives convolved with the source wavelet (see Fig. VI.7). Thus, we can produce partial derivative seismograms by two reciprocal simulations, with a $\mathrm{HC}$ source and a VC source, and the convolution.

Seismic inversion is an underdetermined problem. Therefore, the resolution required by accurate forward simulations and the resolution that can be achieved by inversion are quite different (Sasaki 1989, Pratt et al. 1998). To overcome this difficulty we use a multi-

grid approach: a fine grid for forward simulations, and a coarse one for seismic inversion. If a model parameter is taken as a block, then the virtual source should be considered as a block (see Fig. VI.1). In this cast, the partial derivatives are generated by the block source, and the reciprocal seismograms are obtained from sums of seismograms within the block. Fig. VI.8 shows convolved partial derivative seismograms from a virtual source block and the reciprocity whose receivers are located at the block.

\section{Gradient \& Gauss-Newton method with reciprocity \& convolution}

From the reciprocity and convolution property of the previous section, we can decompose the Jacobian matrix into virtual sources and reciprocal responses:

$$
\begin{aligned}
J_{p, q}^{i} & =\left(V_{p}^{i} * R_{q}^{i}\right) \\
& =\left(\left.\left.V_{p}^{i}\right|_{x} * R_{q_{-} x}^{i}\right|_{x}+\left.\left.V_{p}^{i}\right|_{z} * R_{q_{-} x}^{i}\right|_{z},\left.\left.\quad V_{p}^{i}\right|_{x} * R_{q_{-} z}^{i}\right|_{x}+\left.\left.V_{p}^{i}\right|_{z} * R_{q_{-} z}^{i}\right|_{z}\right),
\end{aligned}
$$

where the superscript $i$ means a location of model parameters and the subscripts $p$ and $q$ represent the source and receiver, respectively. $V$ represents the virtual source wavefields generated by a surface source $p . R$ is the reciprocal wavefields recorded at $i^{\text {th }}$ location of model parameter and generated from $q^{\text {th }}$ receiver location. $\left.\right|_{x}$ and $\left.\right|_{z}$ represent the orientation of recorded wavefields. $x$ and $\_z$ mean the orientation of the source component (see Fig. VI.9). Then the gradient direction can be obtained by

$J_{p, q}^{i} \otimes \Delta d_{p, q}=\left(\left.\left.V_{p}^{i}\right|_{x} * R_{q_{-} x}^{i}\right|_{x}+\left.\left.V_{p}^{i}\right|_{z} * R_{q_{-} x}^{i}\right|_{z},\left.\left.\quad V_{p}^{i}\right|_{x} * R_{q_{-} z}^{i}\right|_{x}+\left.\left.V_{p}^{i}\right|_{z} * R_{q_{-} z}^{i}\right|_{z}\right) \otimes \Delta d_{p, q}$

where $\otimes$ means zero-lag cross-correlation and $\Delta d_{p, q}$ represent the residual at $q^{\text {th }}$ receiver of $p^{\text {th }}$ shot gather. Before cross-correlating the Jacobian and the residual, the residual should be 
convoluted with the source time function used for the reciprocal wavefields $R$. The procedure to compute the gradient direction with explicit calculation of the Jacobian for multi shots is summarized below:

(1) Simulate all forward wave propagations from $p^{\text {th }}$ shot and compute the residuals. Save forward wavefields at $i^{\text {th }}$ location of model parameter and the receivers.

(2) Simulate reciprocal wave propagations from the horizontal and vertical component of $q^{\text {th }}$ receiver. At this step, it is not necessary to use the same source time function as the first step.

(3) Convolute the source time function of step (2) and the residuals from step (1)

(4) Obtain partial derivative wavefields by convoluting wavefields from step (1) and (2).

(5) Calculate the gradient direction by zero-lag cross-correlation the wavefields from step (4) and convoluted residuals from (3).

The explicit calculation of the Jacobian requires $2 \times N+n s h o t$ of wave propagation simulations. Because the convolution is computed in frequency domain, it doesn't affect the computation time much.

In order to take advantage of the Gauss-Newton method, it is required to compute the approximate Hessian matrix. The main drawback of the Gauss-Newton method is that the evaluation of the approximate Hessian requires the storage of the Jacobian that can be quite large for certain problems, and besides, the evaluation of the Jacobian costs huge amounts of the storage (see step 1). However, it turns out that the Jacobian does not have to be computed and stored as a whole. For example, if we were able to divide the Jacobian into sub-matrices we could compute the approximate Hessian as follows:

$$
\mathbf{H}_{a}=\mathbf{J}^{t} \mathbf{J}=\left[\begin{array}{lll}
\mathbf{J}_{1} & \cdots & \mathbf{J}_{\text {nshot }}
\end{array}\right]\left[\begin{array}{c}
\mathbf{J}_{1} \\
\vdots \\
\mathbf{J}_{\text {nshot }}
\end{array}\right]=\sum_{\text {nshot }} \mathbf{J}_{i}{ }^{t} \mathbf{J}_{i}
$$

Therefore, the full Jacobian does not have to exist at one time. The approximate Hessian can be computed by summing a series of elements. In addition to this, the approximate Hessian is symmetric so that it's not necessary to calculate whole elements. Fig. VI.10 shows the parallel implementation of computing symmetric approximate Hessian. Another difficulty of 
the Gauss-Newton method is to solve the inverse of the damped approximate Hessian. In our approach, we use the Parallel Engineering and Scientific Subroutine Library (PESSL) to make better use of the parallel systems.

Because we don't have the full Jacobian, it is not practical to compute the optimal step length explicitly. To compute $\mathbf{J}^{\mathrm{t}} \mathbf{g}^{\mathrm{n}}$ of equation (VI.24), we use a finite-difference approximation:

$\mathbf{J}^{t} \mathbf{g}^{n} \cong \frac{1}{\varepsilon}\left[\mathbf{F}\left(\mathbf{m}^{n}+\varepsilon \mathbf{d}^{n}\right)-\mathbf{F}\left(\mathbf{m}^{n}\right)\right]$,

where $\varepsilon$ is a sufficiently small value.

\section{J Numerical examples}

In this section we examine the effectiveness of the methodology described above. Fig. VI.11 shows the simple model that has a small block of anomalous density. The model has $160 \mathrm{~m}$ $\mathrm{x}$-extent and $80 \mathrm{~m} \mathrm{z}$-extent. The grid spacing and the time step are $0.4 \mathrm{~m}$ and $60 \mu \mathrm{s}$. The first time derivative of the Gaussian function with a frequency of $100 \mathrm{~Hz}$ is used as the source time function. The media properties are given in Fig. VI.11. A seismic source and 10 receivers are located below the free surface. The gradient directions are obtained from the acoustic and elastic models which the free surface and the absorbing boundary conditions are respectively applied to. Because the free surface boundary condition would make stronger amplitude of the surface waves than that of the reflected waves, they would be a detriment to the gradient directions.

In the first example, the free surface boundary condition is applied. In particular, the gradient directions from the acoustic model (Figs. VI.12a-c), unlike as those from the elastic model (Figs. VI.12d-f), don't show the artifacts from the interferences between the reflected and the surface waves. Figs. VI.12a and VI.12d are computed from the adjoint state method, Figs. VI.12b and VI.12e from the gradient method, and Figs. VI.12c and VI.12f from the Gauss-Newton method. The gradients of Figs. VI.12b and VI.12e are blurred more than those of Figs. VI.12a and VI.12d, respectively. This is because of step (2) and (3) from the previous section. In these steps, we convolute the original fields with another source time function. Thus, if we use limited frequency band of the source time function, then we can obtain bandpass filtered gradients and it will be the topic of future works. Although the directions are not well resolved with this gradient method, the preconditioning by the 
approximate Hessian matrix can greatly improve the resolving power (see Figs. VI.12c and f). Also, the artifacts from the surface wave are effectively removed. We also observe the role of the S wave which can improve the horizontal resolution of the anomaly.

Fig. VI.13 shows the gradient directions from the model whose free surface boundary condition is the absorbing boundary condition model. The other configurations are the same as above. Because the surface waves are free of distress to the acoustic problem, the results from the acoustics are same as the above. However, in the case of the elastic problem, because the surface waves are dominant at near surface, the improvement by exploiting the absorbing boundary condition at the surface is remarkable (compare Figs.VI.12d-f and Figs. VI.13d-f). The artifacts from the surface waves are eliminated even in the results from the adjoint state or the gradient method. The advantages of the GaussNewton method and the $\mathrm{S}$ waves are also noticeable. Fig. VI.14 shows the gradients directions from the single, especially, vertical component data of the elastic problem. Because of the lack of the information, these directions are not clear as much as Figs. VI.13e and VI.13f but still show the superiority over those from the acoustics (Fig. VI.13b and VI.13e).

For waveform inversions, we added 2 shot gathers whose sources were located at the left and right receiver positions of the source given in Fig. VI.11. Each shot gather has 6 receivers whose vertical components are only used for inversions. The inversion results are shown in Fig. VI.15. The gradient method stopped after 7 iterations and the Gauss-Newton method after 18 iterations. Note that even the first iteration result of the Gauss-Newton method gives more accurate values than the final result of the gradient method. Also, the estimated densities from the Gauss-Newton are almost close to the original model (Fig. VI.15d). We can calculate the Jacobian explicitly and don't store the Jacobian as a whole, but it is still heavy to make use of the approximate Hessian matrix for high-resolution inversion results. We use 3,600 model parameters $(60 \times 60$ nodes $)$ for this example and the required memory storage for the Hessian is about $50 \mathrm{MB}$ although we utilize the symmetry of the Hessian. If we consider $300 \times 300$ nodes of model parameters, the required memory, only for the Hessian, is about 30 GB. However, it is not necessary for the resolution of inverse problems to be as high as that of forward simulations (Sasaki, 1989, Pratt et al., 1998). Thus, block parameterization, which consists of 1,600 $(40 \times 40)$ blocks and whose 
required memory is only about $10 \mathrm{MB}$, is introduced to the Gauss-Newton inversion (Fig. VI.16) and this covers 32,000 model parameters $(200 \times 160$ nodes $)$ which would be required 3.8 GB of memory storage. The block parameterized inversion stopped after 17 iterations and also shows a good approximation of the true model. The Gauss-Newton method shows great convergence rate and block parameterized Gauss-Newton method also converged very well (Fig. VI.17).

In the next example, we investigate $\mathrm{P}$ wave velocity inversion with a 4-layer model (Fig. VI.18). For simplicity, the density and the $\mathrm{S}$ wave velocity are assumed to be constants. The source wavelet has a dominant frequency of $30 \mathrm{~Hz}$ and 3 shot gathers are used with source locations of $-210,0$ and $210 \mathrm{~m}$. Each shot gather has 10 receivers from 630 to $630 \mathrm{~m}$ with the spacing of $105 \mathrm{~m}$ and the minimum offset is $210 \mathrm{~m}$. The depth of sources and receivers is $0 \mathrm{~m}$ and all sides of the model are surrounded by the absorbing layers. The model parameters consist of 1,600 $(40 \times 40)$ blocks which covers $400 \times 130$ nodes. The true velocity model is given in Fig. VI.18a. The velocity of the starting model increases with depth (Fig. VI.18b). Two regularization schemes are applied: the damped least-squares method (Fig. VI.18c) and the Laplacian constraints (Fig. VI.18d). The effects of the Laplacian constraints that make the model parameters update smoothly can be easily observed (Fig. VI.18d). Both results succeed in recovering the true model (Fig. VI.18a) except horizontal edges of them. From the source and receiver configurations, we recognize that the seismic rays haven't contributed effectually toward the inversions, which passed through those areas. Thus, this makes spurious results at the edges. Fig. VI.19 shows velocity profiles of the mean values from horizontal distances of $-420 \sim 420 \mathrm{~m}$ for each depth. As a result, it is clear that this approach can restore the true velocity model precisely. Now, assume that we have a priori information suggesting a layered structure. But we don't know the exact location of a fault. Figs. VI.20a and VI.20b show the true model and a starting model, respectively. We use 5 shot gathers whose source locations are from 420 to $420 \mathrm{~m}$ with the spacing of $210 \mathrm{~m}$. Each shot gather has 10 receivers which are the same as above. The Laplacian constraints are applied to the inversion stage (Figs. VI.20c and VI.20d). From the assumption, as we know the true values of the model at certain positions, we set the model parameters fixed below at -630 and $630 \mathrm{~m}$ (Fig. VI.20d). To reduce the residuals to $0.05 \%$ takes 20 iterations for Fig. VI.20c and 10 iterations for Fig. 
VI.20d. There are some artifacts near the layer boundaries which may result from the discordance between the block parameterization and the velocity structure. From these results, however, we could figure out the location and the extension of the fault. The seismograms are shown in Fig. VI.21, which are generated from the true model, a starting model, the final model (Fig. VI.20d) and the residual between those form the true model and the final. The final results still have some high frequency mismatches which can be probably accounted for the block parameterization rather than for the performance of the inversion scheme.

\section{K Summary}

A 2-D elastic seismic waveform inversion based on time domain approach has been developed to obtain physical parameters of the Earth from prestack seismic reflection data. Seismic wavefields are computed by the velocity-stress staggered-grid finite difference method (Levander, 1988; Graves, 1996). The algorithm calculates the partial derivative wavefields explicitly by using the seismic reciprocity and convolution, and produces the approximate Hessian matrix for the Gauss-Newton inversion scheme. In order to increase the convergence rate and stability of the inversion scheme, we use the damped-least squares, the Laplacian constraints method. Because this approach requires extremely extensive computations and memories, we utilize this work based on scalable massively parallel computers with MPI and PESSL. 


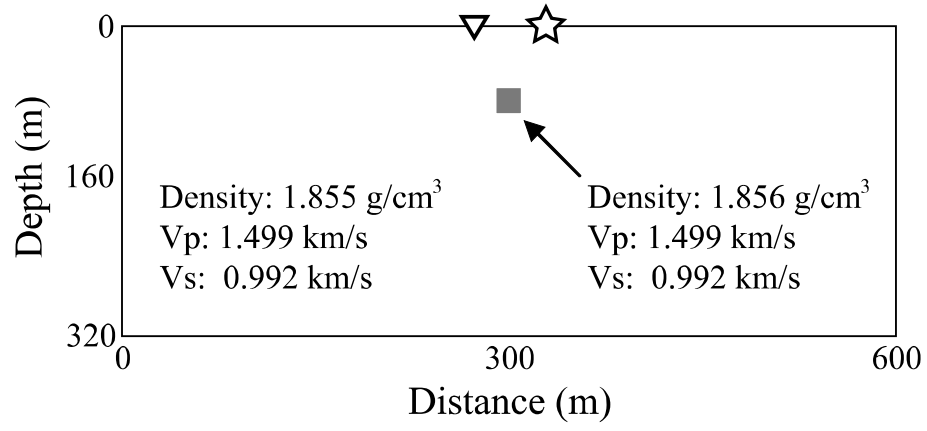

Fig. VI.1 Schematic diagram of a homogeneous model with a perturbed density block. Star and triangle denote seismic source and receiver, respectively.

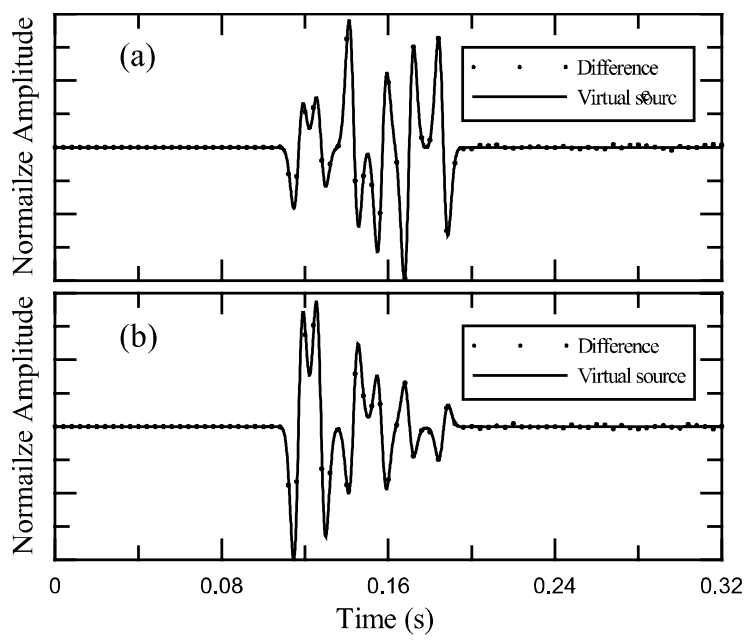

Fig. VI.2 Partial derivative seismograms (a) horizontal component; (b) vertical component seismogram. 


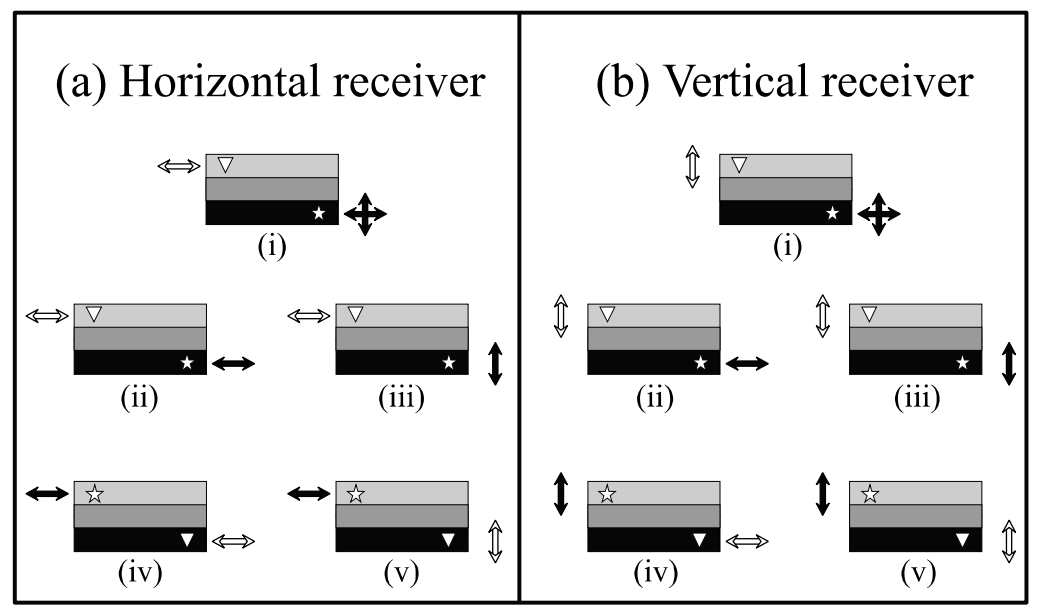

Fig. VI.3 Schematic diagram of the reciprocity theorem. (a) reciprocal reconstruction of the horizontal motion and (b) vertical motion. Star and triangle denote seismic source and receiver, respectively. White and vertical arrows mean receiver and source orientation, respectively.

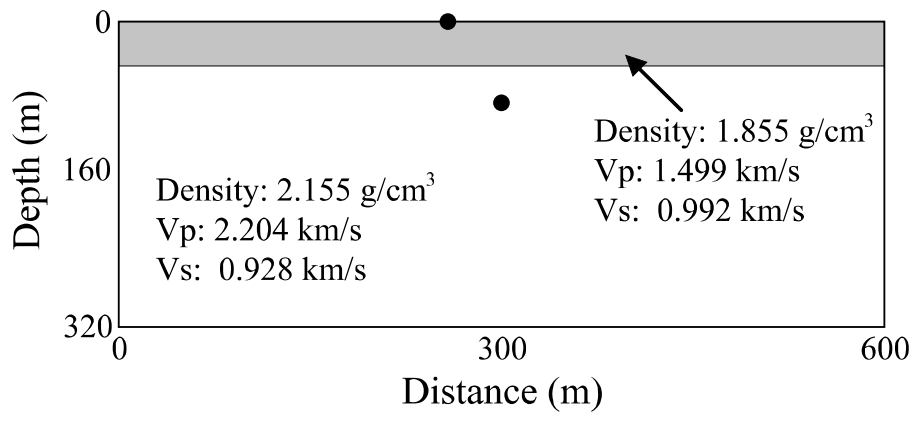

Fig. VI.4 Schematic diagram of the two layer model. The dot denotes a pair of source/receiver location. 

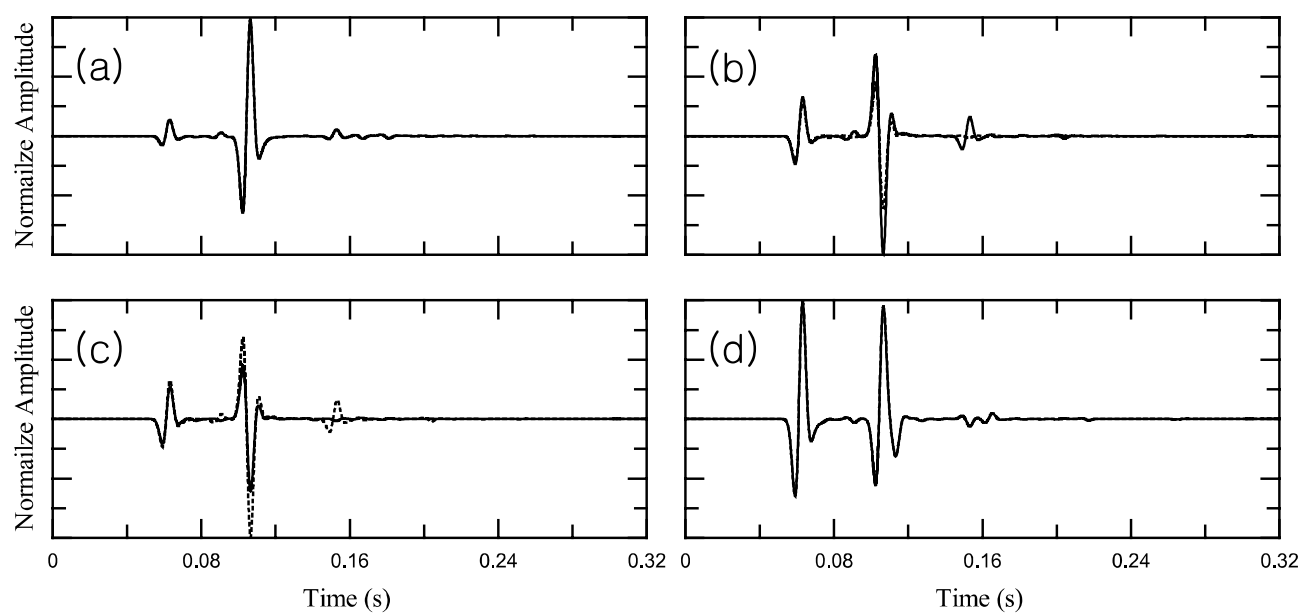

Fig. VI.5 Synthetic seismograms. (a) and (b) are generated from the horizontal source; (c) and (d) from the vertical source. The receiver orientations of (a) and (c) are horizontal and those of (b) and (d) vertical, respectively. Dotted lines obtained from a source on the surface and solid lines from a source inside the medium. The discrepancies of (b) and (c) show that the reciprocity relation is only valid if both of the reciprocal location and the reciprocal orientation match.

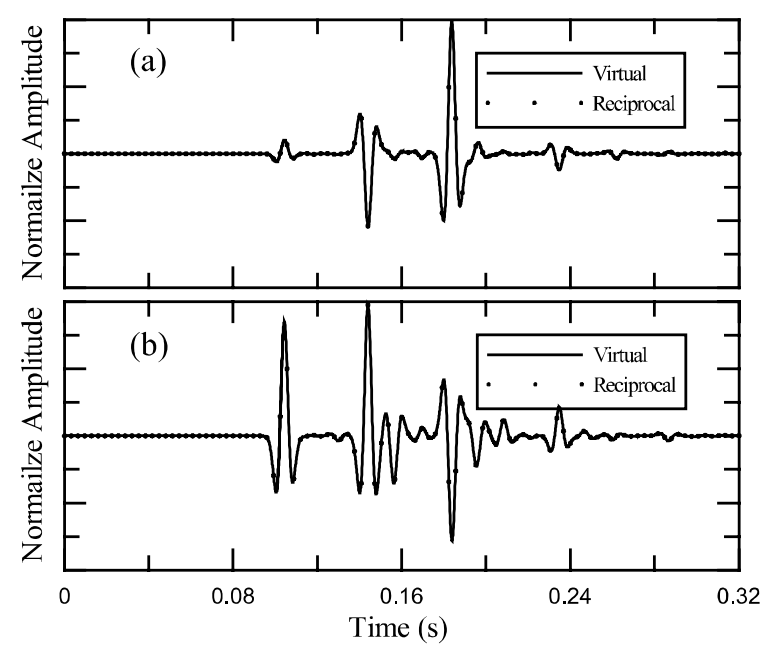

Fig. VI.6 Partial derivative seismograms from the virtual source and the reciprocity relation. (a) horizontal component; (b) vertical component seismogram. 


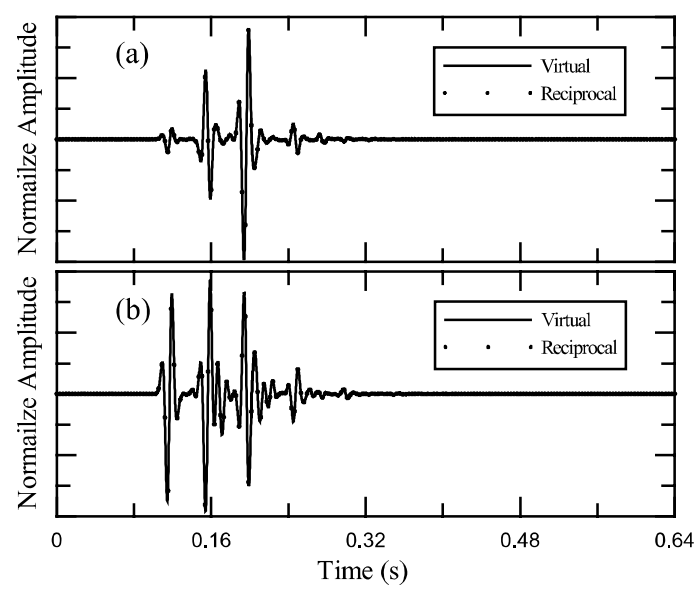

Fig. VI.7 Convolved partial derivative seismograms from the virtual source and the reciprocity relation. (a) horizontal component; (b) vertical component seismogram.

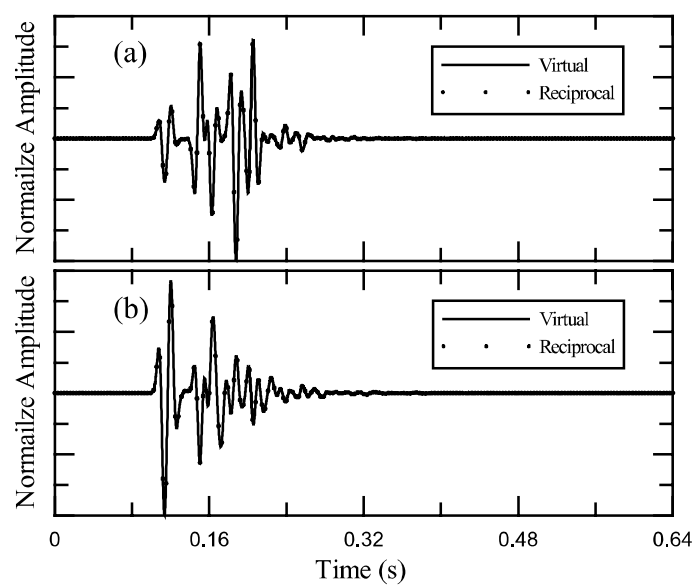

Fig. VI.8 Convolved partial derivative seismograms from the virtual source block and the reciprocity relation. (a) horizontal component; (b) vertical component seismogram. 

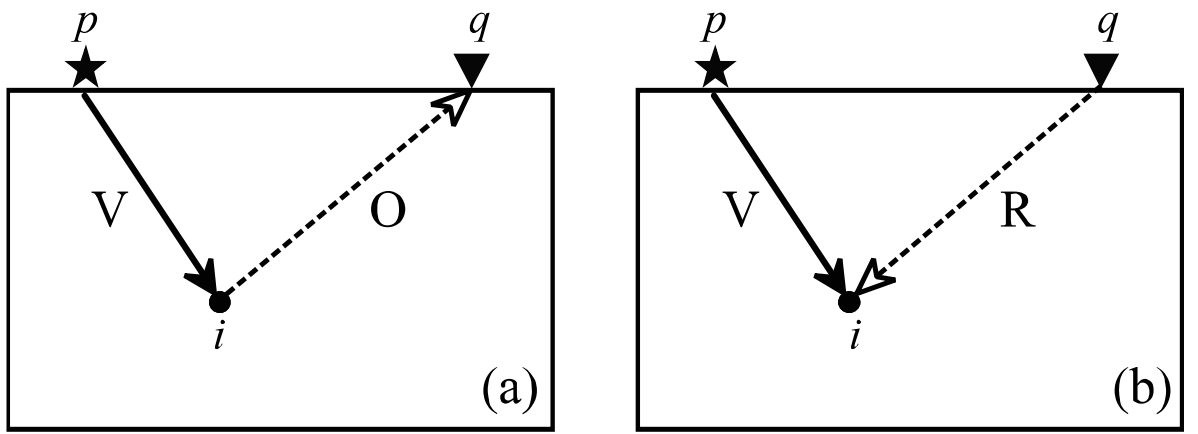

Fig. VI.9 Schematic diagram for general reciprocal relation for partial derivative wavefields. (a) realistic wave propagation; (b) reciprocal wave propagation. $p, q$ and $i$ represent the indexes of a source, receiver and media parameter, respectively. V, O and R mean the down going wave, partial derivative wave and reciprocal wave, respectively.

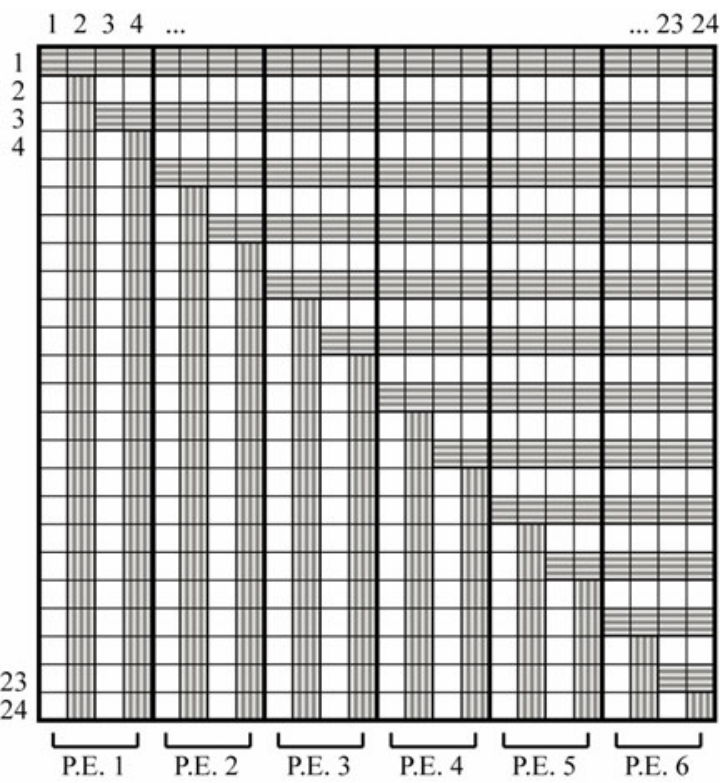

Fig. VI.10 Representation of the parallel implementation for calculating symmetric approximate Hessian matrix. P.E means each processing element. It only needs to calculate shaded elements and this distributes computation and memory loads to each P.E. evenly. 


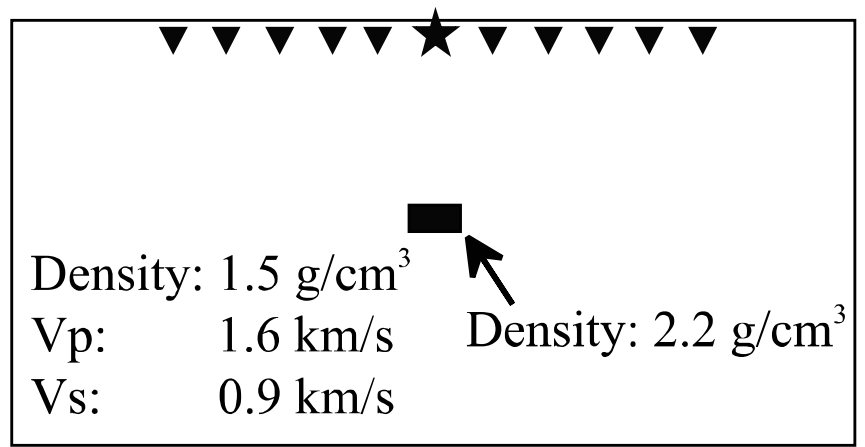

Fig. V.11 Simple model for examining the gradient directions.
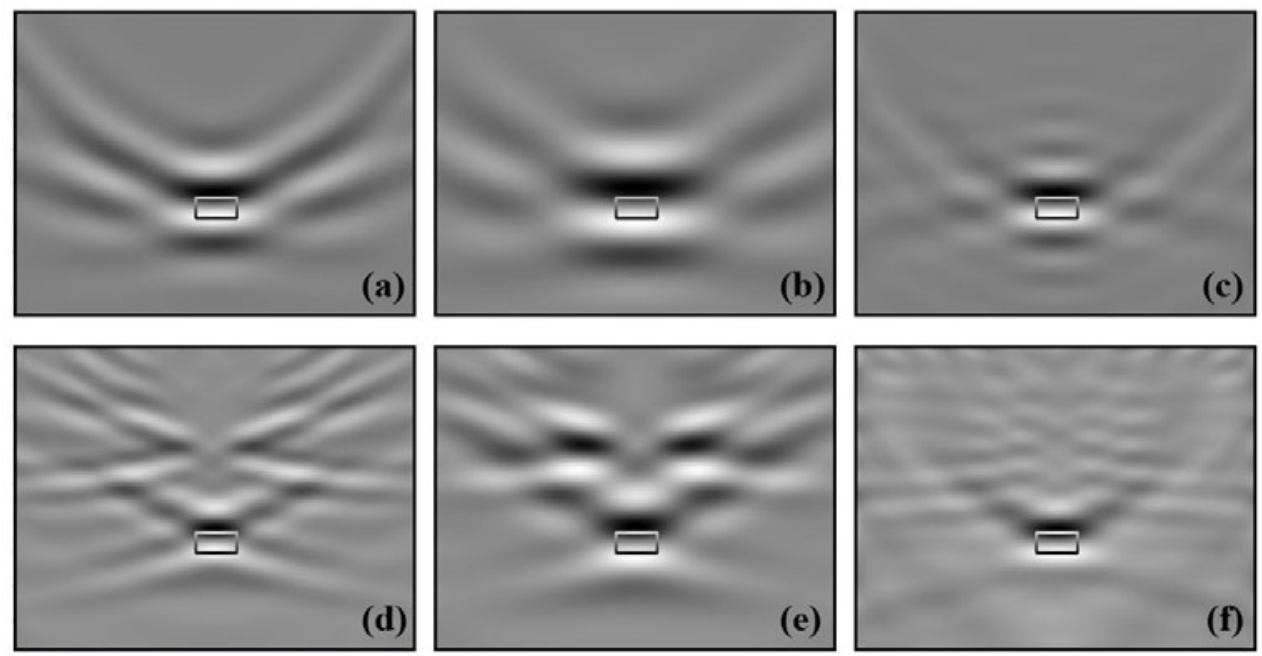

Fig. V.12 Gradient directions computed from the free surface boundary condition model shown in Fig. VI.11. The rectangular boxes represent the anomalous block. (a)-(c) are from the acoustic model and (d)-(f) from the elastic model. (a) and (d) are computed by the adjoint state method, (b) and (e) by the gradient method, and (c) and (f) by the Gauss-Newton method, respectively. 

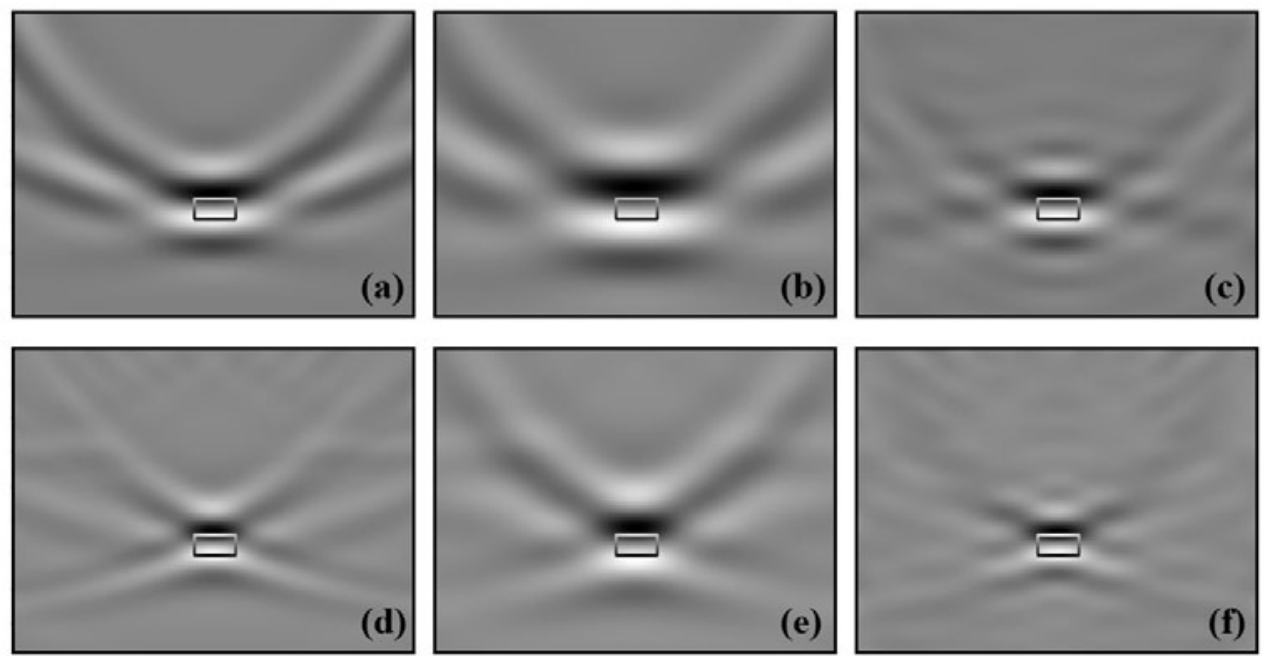

Fig. VI.13 Same as Fig.VI.12 but using the absorbing boundary condition at the Earth's surface.
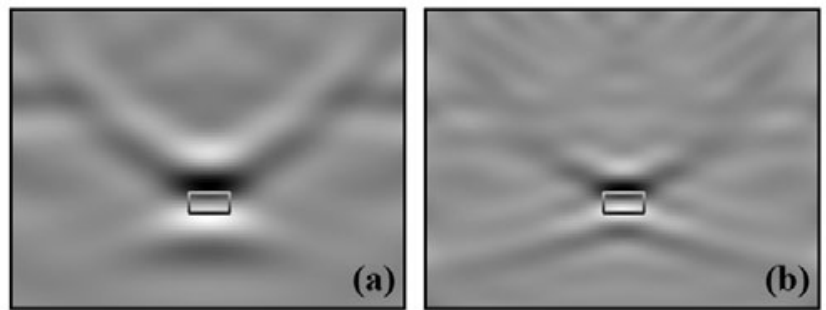

Fig. VI.14 Gradient directions computed from the vertical component of the elastic model shown in Fig. VI.11. The absorbing boundary conditions are applied to the computational boundary. The rectangular boxes represent the anomalous block. (a) is obtained from the gradient method and (b) from the Gauss-Newton method, respectively. 


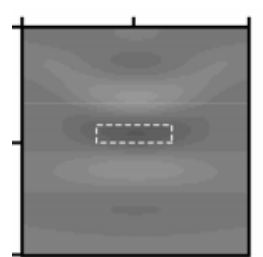

(a)

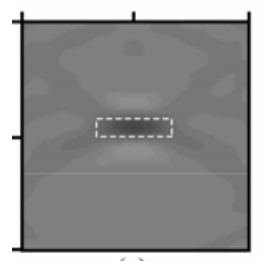

(c)

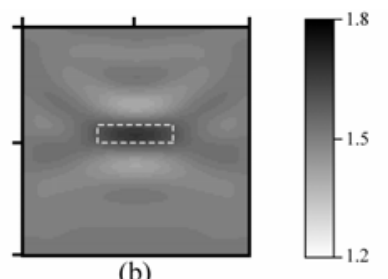

Density $\left(\mathrm{g} / \mathrm{cm}^{3}\right)$

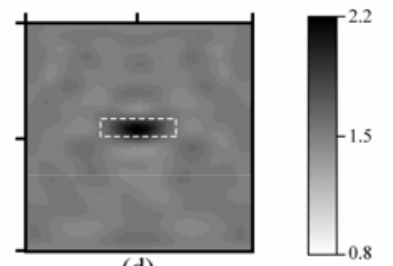

(d)

Fig. VI.15 Inversion results for the elastic model shown in Fig. VI.11. (a) The first iteration result and (b) the final result from the gradient method. (c) The first iteration result and (d) the final result from the GaussNewton method.

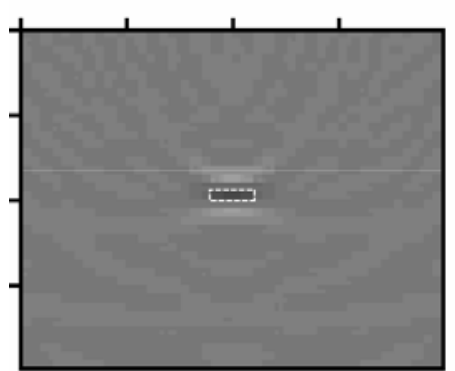

(a)

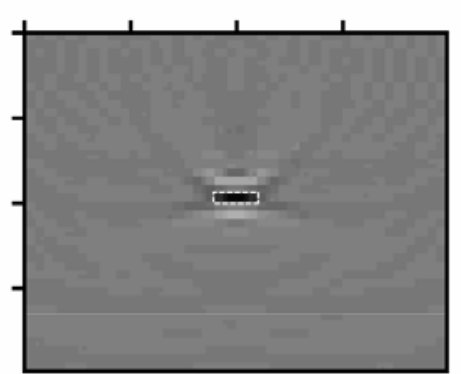

(b)

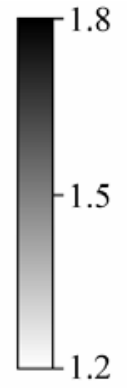

Density $\left(\mathrm{g} / \mathrm{cm}^{3}\right)$

Fig. VI.16 Inversion results of the Gauss-Newton method for the block parameterization. (a) The first iteration result and (b) the final result. 


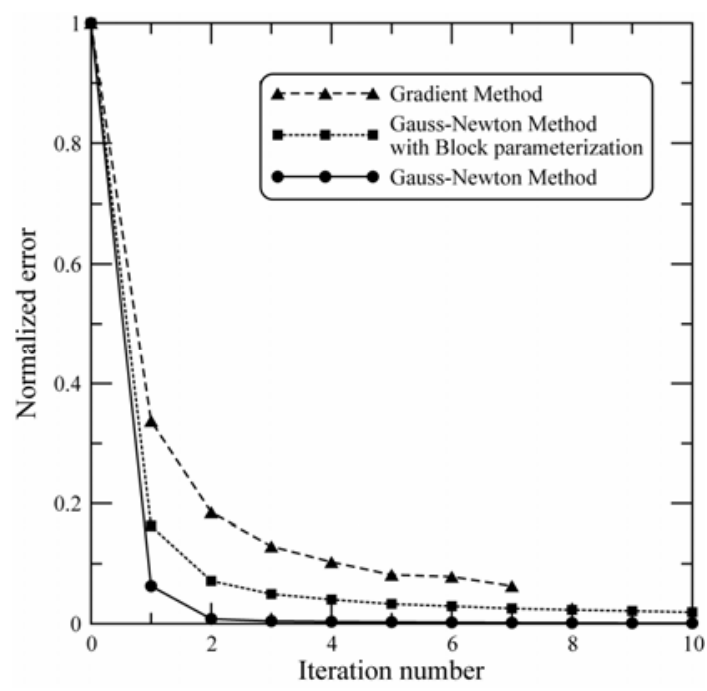

Fig. VI.17 Normalized errors from the inversion steps for the model shown in Fig. VI.11.

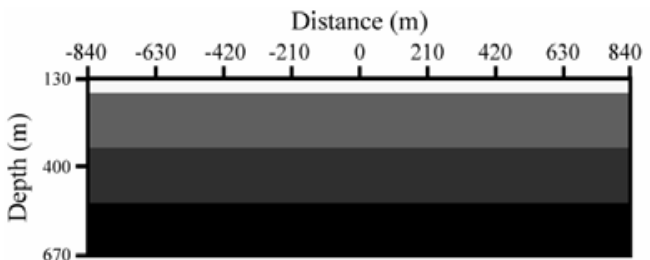

(a)

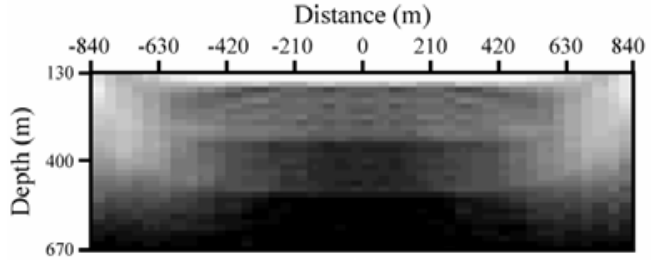

(c)

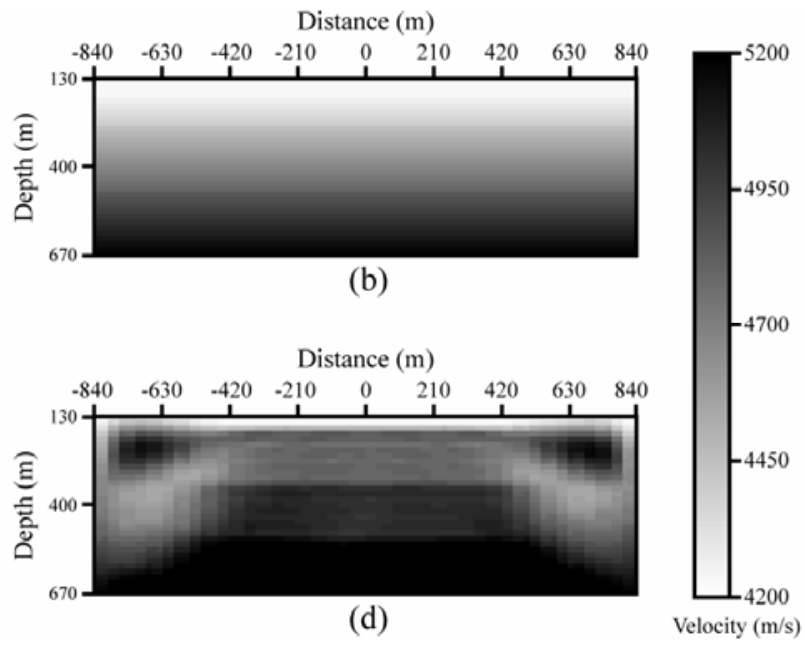

(d)

Fig. VI.18 P wave velocity inversion results for a layered model. (a) True model. (b) Starting model. (c) Inversion result from the damped least-squares. (d) Inversion result from the Laplacian constraints. Horizontal locations of the source are $-210,0$ and $210 \mathrm{~m}$ and those of receivers from -630 to $630 \mathrm{~m}$ with the spacing of $105 \mathrm{~m}$, respectively. Vertical locations of source and receiver are $0 \mathrm{~m}$. There are some artifacts in horizontal edges of (c) and (d) because seismic rays don't go through those areas. 


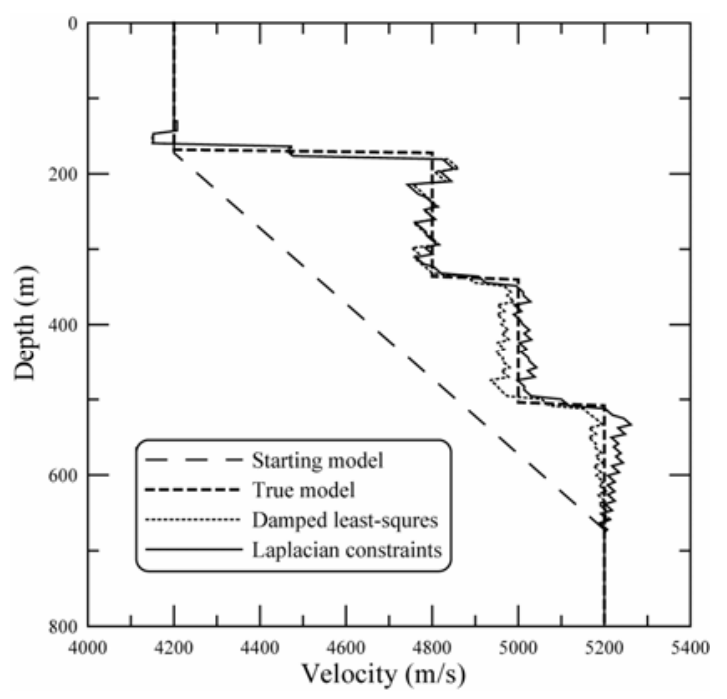

Fig. VI.19 P wave velocity profiles from Fig. VI.18. Velocities are obtained by taking the mean values from horizontal distances of $-420 \sim 420 \mathrm{~m}$ for each depth.

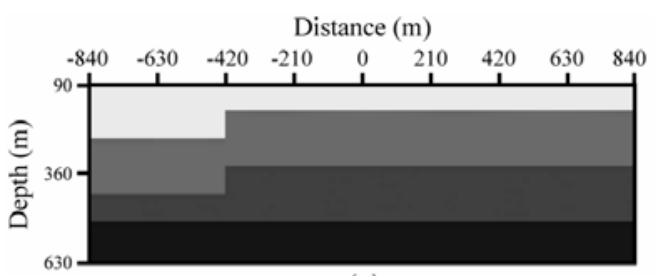

(a)

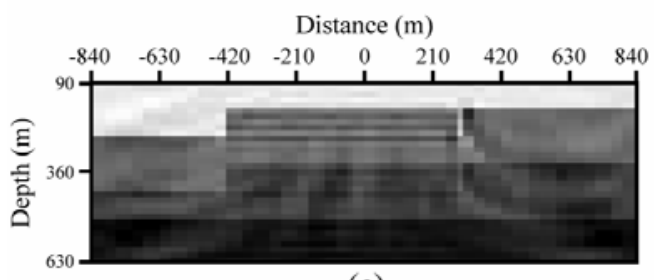

(c)

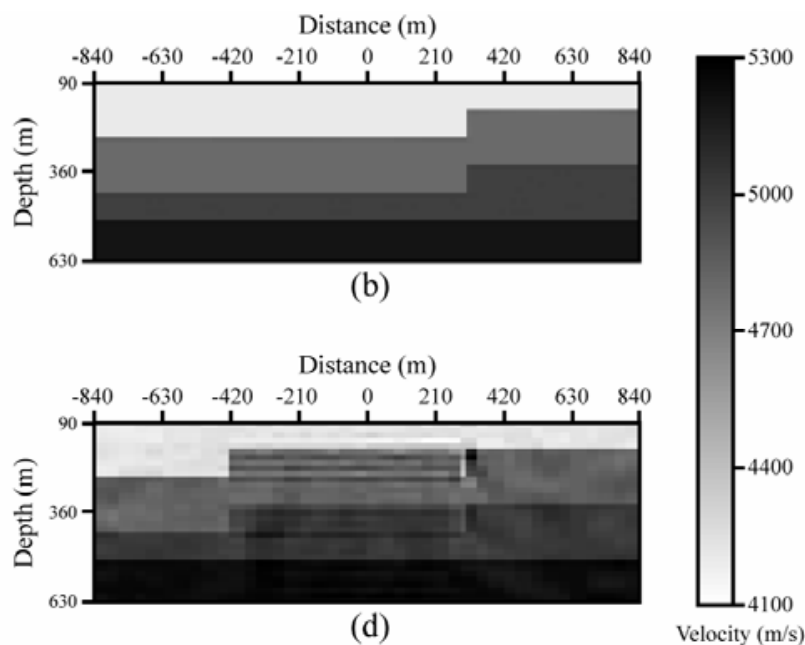

(d)

Fig. VI.20 P wave velocity inversion results for a fault model. (a) True model. (b) Starting model. (c) Inversion result from the Laplacian constraints. (d) Inversion result from the Laplacian constraints with fixed values below -630 and $630 \mathrm{~m}$. Horizontal locations of the source are $-420,-210,0,210$ and $420 \mathrm{~m}$ and those of receivers from -630 to $630 \mathrm{~m}$ with the spacing of $105 \mathrm{~m}$, respectively. Vertical locations of source and receiver are $0 \mathrm{~m}$. 


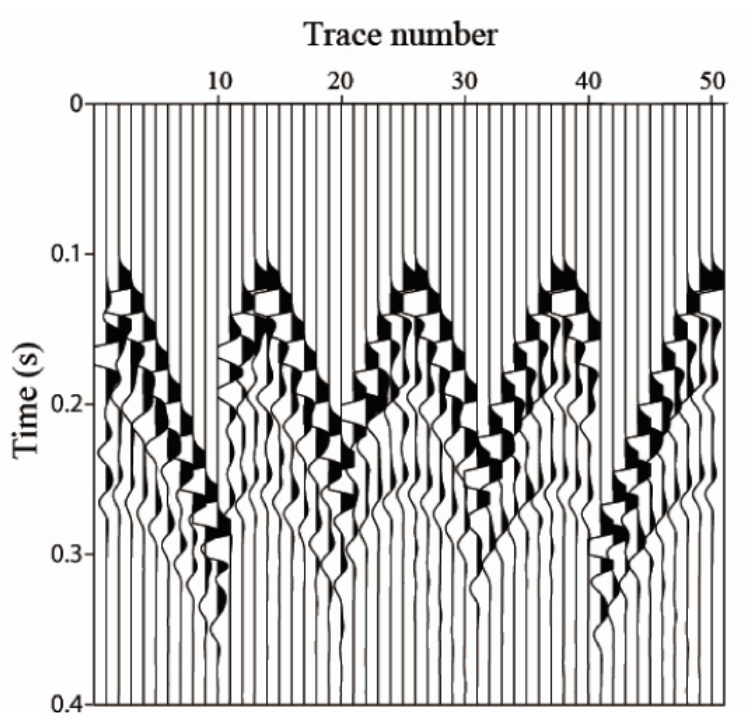

(a)

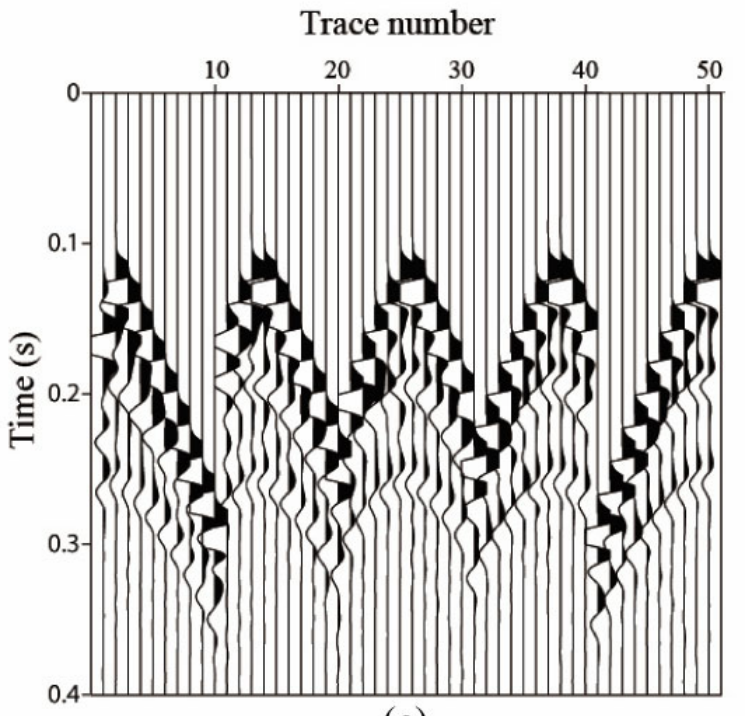

(c)

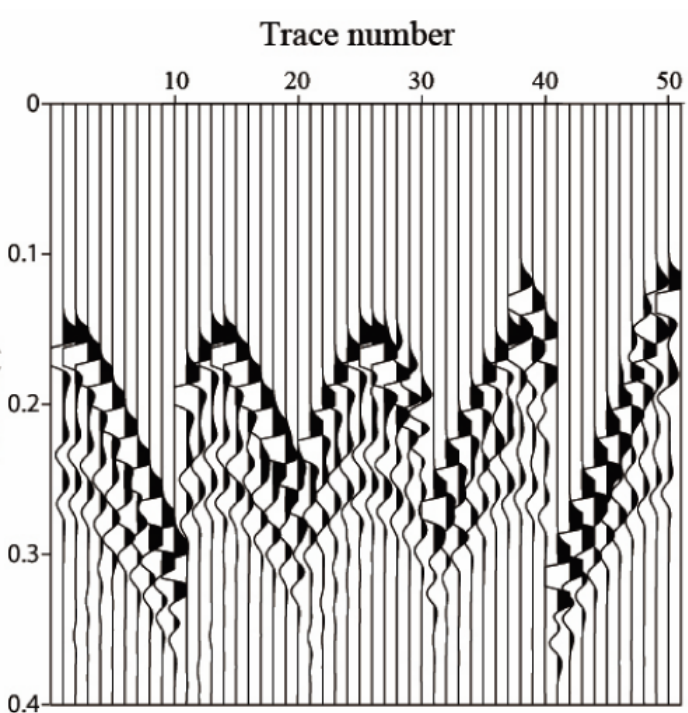

(b)

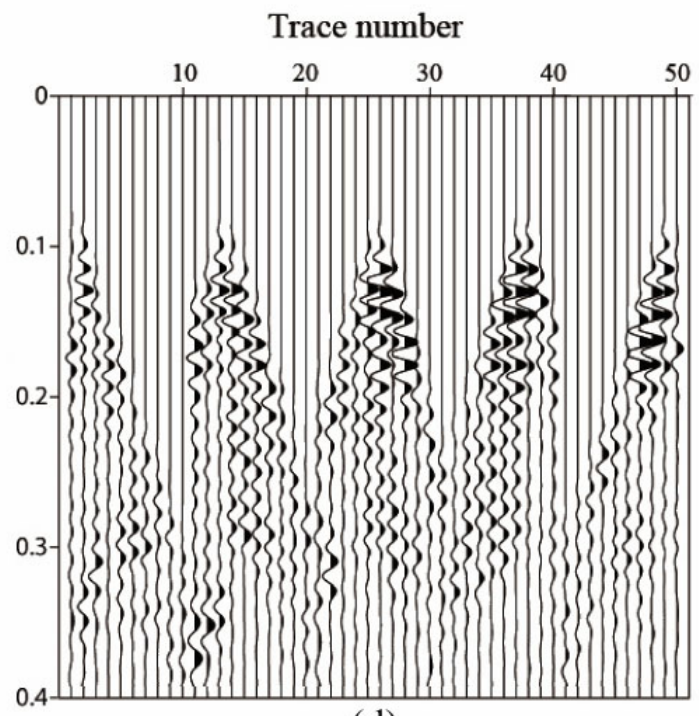

(d)

Fig. VI.21 Comparison of vertical component seismograms: (a) from the true model, (b) a starting model, (c) the final model shown in Fig. VI.20d and (d) the residuals between (a) and (c). The direct arrivals are removed by subtracting the data calculated from the homogeneous model. The scaling factor of the residual is 10 relative to the others. 


\section{Compare Predicted and Observed Fracture Network Properties (Subtask 4.6)}

We performed the seismic waveform inversion of a land seismic reflection survey data from Harrison co., Indiana. The acquisition geometry consisted of 30 vertical component geophone arrays on each side of the shot point. The nearest and farthest offsets of the data were 990 and $7370 \mathrm{ft}$ (297 and 2,211 m). The energy source was the Vibroseis source and an inline array of 4 vibrators had $35 \mathrm{ft}$ pad to pad spacing. The sweep length, recording length and frequency was $12,16 \mathrm{~s}$ and $10-90 \mathrm{~Hz}$, respectively. The shot point and receiver intervals were $220 \mathrm{ft}(66 \mathrm{~m})$. The recorded data were correlated with the Vibroseis source. We used $0.6 \mathrm{~s}$ of data for the inversion, whose recording length was $4.0 \mathrm{~s}$ after the correlation. The data sampling interval was $4 \mathrm{~ms}$, however, the data was resampled to 0.3 $\mathrm{ms}$ to accommodate the synthetic data from the finite-difference method.

We applied several preprocessing steps to the real data before the inversion.

1. Before correlating the data, we applied the Vibroseis whitening (Coruh and Costain, 1983) for equalizing frequency contents of the data.

2. After correlating the data with the Vibroseis source, because this inversion algorithm is based on 2-D but the real data are obtained from a 3-D medium, two steps of 3-D to 2-D conversions were performed (Crase et al., 1990; Crease et al., 1992; Červený, 2001).

- The geometrical spreading correction for approximating amplitudes of spherical spreading waves to those of cylindrical spreading waves was accomplished by multiplying field data by $t^{1 / 2}$.

- A convolution with $t^{-1 / 2}$ was performed to transform the point source responses to the line source responses.

3. Because this inversion focuses on the reflected wavefields, we removed the direct-arrivals by dip-filtering and the first-arrivals by muting from the real data. This step was also applied to the synthetic data by differencing with initial model responses. 
For the inversion, we used 10 shot-gathers with an offset range from 990 to $3630 \mathrm{ft}$ (from 297 to 1089 m). The acquisition geometry is shown in Fig. VII.1. The total traces used for the inversion was 110. Fig. VII.2 shows all preprocessed shot records. We made a simple layered model as the initial model from the well logs which were a density log (Fig. VII.3) and sonic log. S velocities are approximated by Poisson's ratio with respect to rock types obtained from well logs.

In this work, we didn't attempt to estimate source functions although the estimation of source functions would affect the quality of the seismic waveform inversion. Instead, we consider an autocorrelated pilot sweep as a source function for the real data, as this wavelet is usually assumed to be a source function for Vibroseis data (Brötz et al., 1987; Ziolkowski, 1991). However, this assumption is generally wrong because, in fact, each shot-gather has different source characteristics due to varying ground conditions under the source. Obviously, our estimations for the source function are crude, and more work on the source estimation is necessary.

In this experiment, our objective was to verify whether seismic waveform inversion could estimate physical parameters of specific zone of interest. Our data set, however, contained little information about the zone shallower than $2200 \mathrm{ft}$ for which we have access to the density and sonic logs. Because the inversion algorithm is based on reflected waves not refracted waves, the information content is higher at near offset data. The redundancy of reflected waves would be necessary to guarantee the convergence of the seismic waveform inversion, like as the stacking procedure in usual seismic data processing. For this experiment, however, only few traces of each shot-gather delivered essential information of the reflections to the inversion. With this acquisition geometry, it turns out that this model can hardly be inverted even for noise-free synthetic data.

Fig. VII.4 shows synthetic seismograms obtained from different acquisition geometries for the same geologic system. The geometry of Fig. VII.4a is the same as that of the real data (offset range: 990-3630 ft; geophone spacing: $220 \mathrm{ft}$ ) and the near offset distance and geophone spacing of Fig. VII.4b is half of the real data (offset range: 440-2530 $\mathrm{ft}$; geophone spacing: $110 \mathrm{ft}$ ). As mentioned above, the reflected waves are strong in near 
offset data and consistent in closer geophone spacing. Table VII.1 shows the comparison of the acquisition geometry with those used in previous inversion studies.

Table VII.1. A comparison of acquisition geometries used in inversion studies.

\begin{tabular}{cccccc}
\hline & $\begin{array}{c}\text { Zone of } \\
\text { interest }(\mathrm{m})\end{array}$ & $\begin{array}{c}\text { Offset } \\
\text { range }(\mathrm{m})\end{array}$ & $\begin{array}{c}\text { Geophone } \\
\text { spacing }(\mathrm{m})\end{array}$ & Medium & $\begin{array}{c}\text { Inversion } \\
\text { method }\end{array}$ \\
\hline $\begin{array}{c}\text { This work } \\
\text { Crase et al. } \\
\quad(1990)\end{array}$ & $80-1,200$ & $100-1,575$ & 12.5 & Elastic & Gradient \\
$\begin{array}{c}\text { Crase et al. } \\
(1992)\end{array}$ & $250-1,700$ & $125-850$ & 25 & Elastic & Gradient \\
$\begin{array}{c}\text { Hicks and Pratt } \\
(2001)\end{array}$ & $100-600$ & $45-620$ & 12.5 & Acoustic & $\begin{array}{c}\text { Gradient } / \\
\text { Newton }\end{array}$ \\
\hline
\end{tabular}


(a)

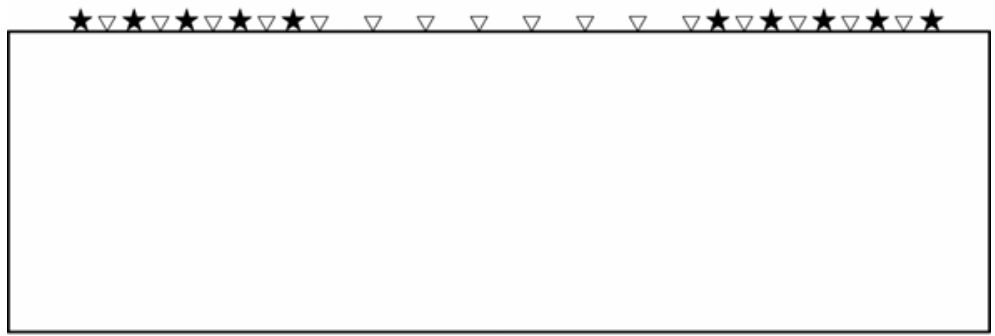

(b)

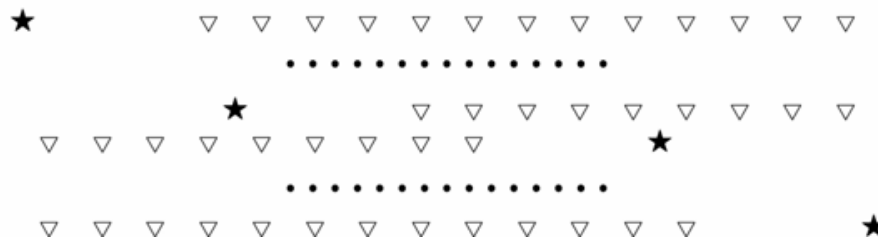

Fig. VII.1 Schematic diagram of the acquisition geometry. (a) Location of sources and receivers. (b) Acquisition geometries for each shot-gather. 


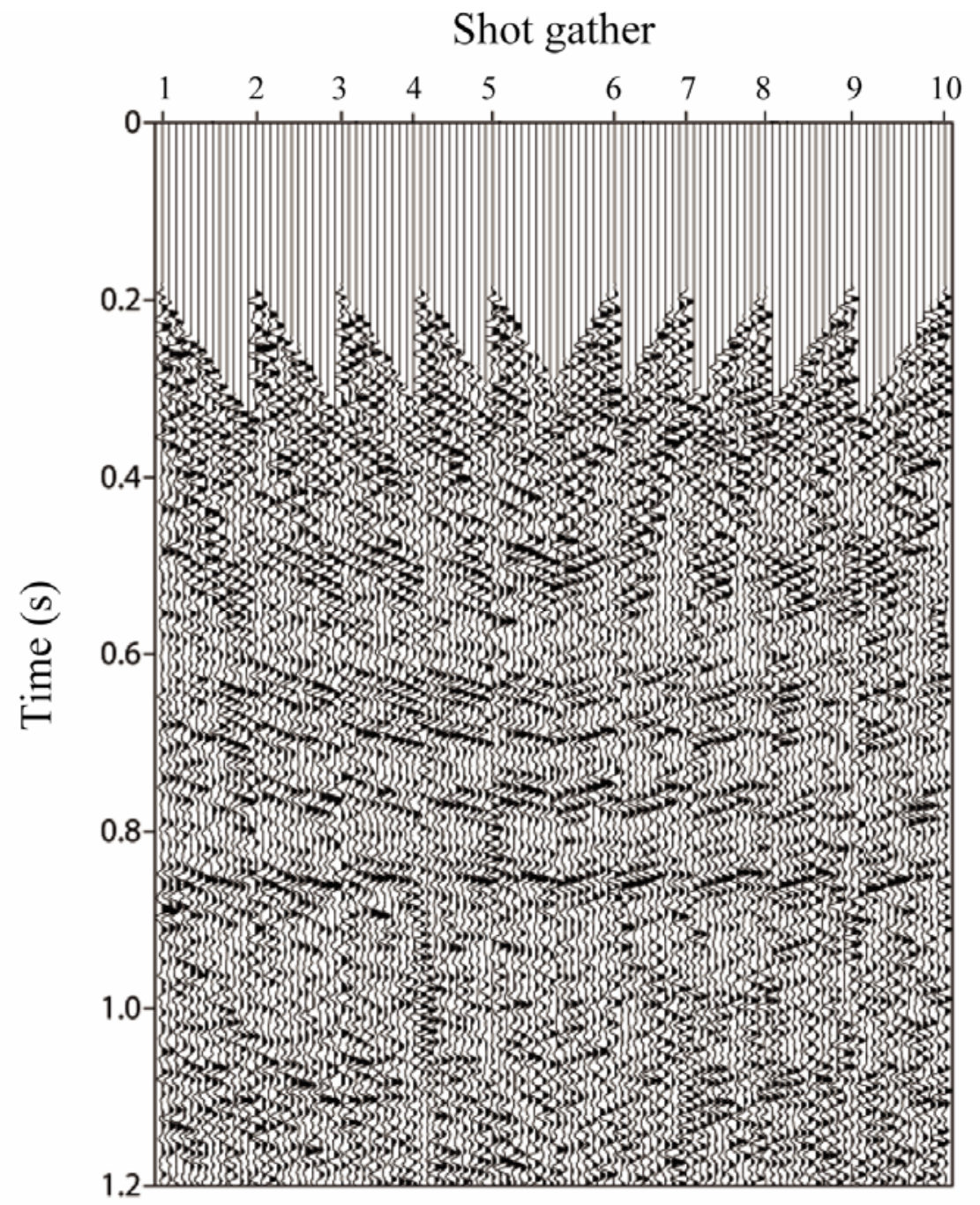

Fig. VII.2 Shot-gathers for the seismic waveform inversion after the application of preprocessing steps described in the text. $0.6 \mathrm{~s}$ of the data were used in the inversion. Automatic gain control (AGC) was applied to this plot. 


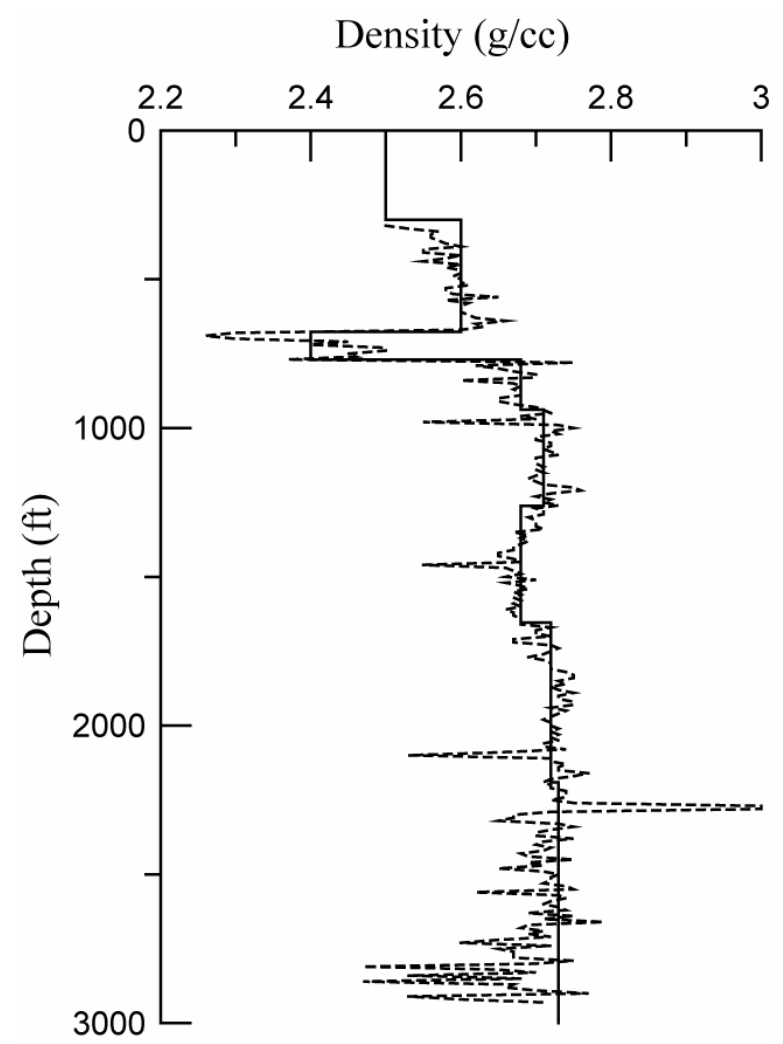

Fig. VII.3 Density log (dashed line) and approximate density model (solid line) for seismic inversion. 


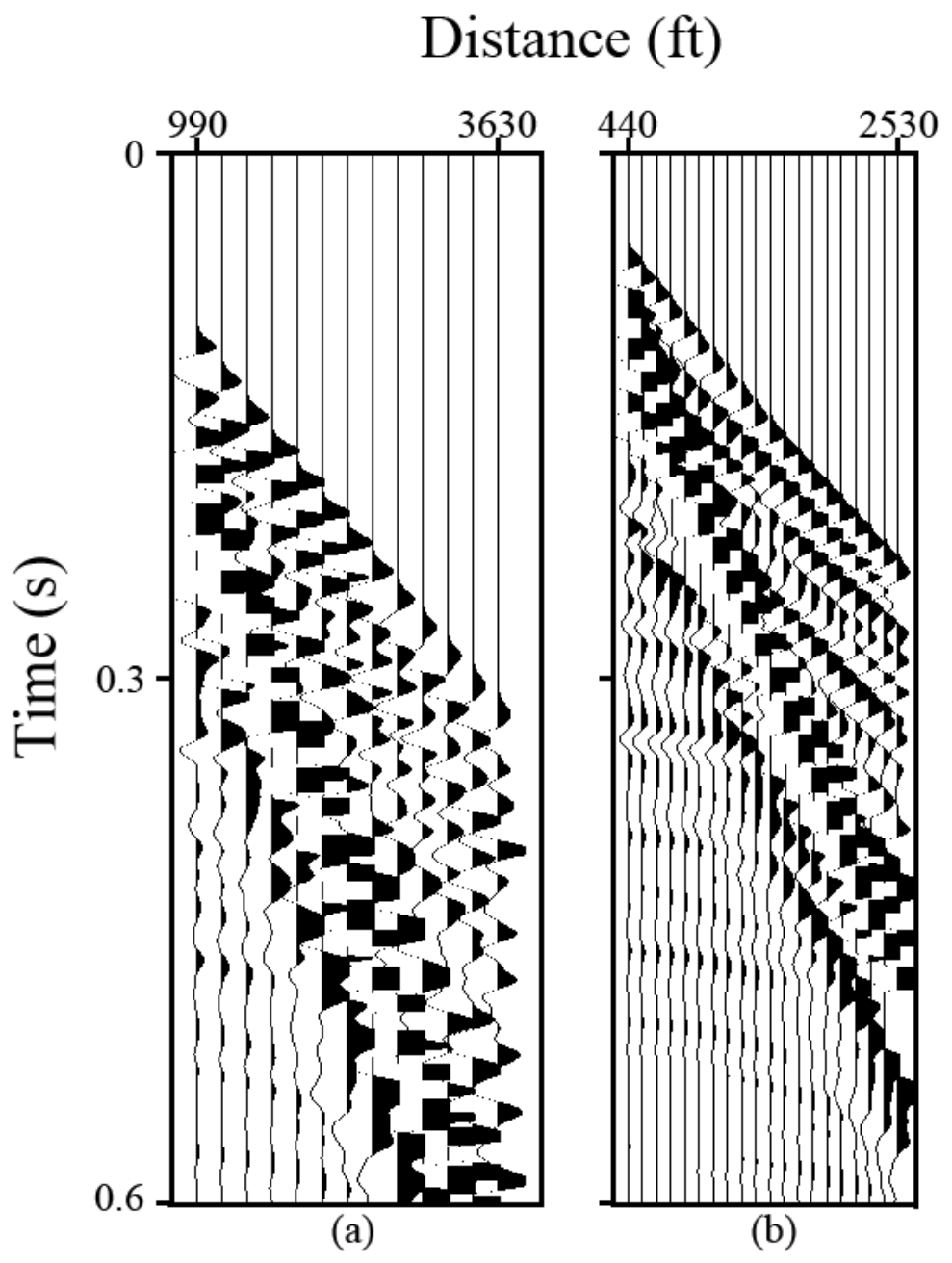

Fig. VII.4 Synthetic seismogram from the initial model constructed based on density log (Fig. 3) and sonic log. (a) Seismogram from the same acquisition geometry as the real data (offset range: 990-3630 ft; geophone spacing: $220 \mathrm{ft}$ ). (b) Seismogram from half near offset distance and geophone spacing of the real data (offset range: $440-2530 \mathrm{ft}$; geophone spacing: $110 \mathrm{ft}$ ). 


\section{Completing the Project in Phase III}

In Phases I and II, we have developed our approach and tested its computational viability. What remains for Phase III is to demonstrate its robustness and accuracy in a realistic situation. The steps we shall take to do this are outlined as follows.

\section{Task 5 Demonstrate SEFD in the Laconia Field of Central Harrison County}

The following describes the tasks with a justification/explanation for each.

Subtask 5.1: Determine the best choice of model parameters to be obtained via the seismic imaging/inversion

The quality and efficiency of the seismic imaging in our SEFD procedure depends on the specific set of variables chosen. One of the following sets of variables needed to characterize the elastic isotropic medium will be chosen:

1. mass density $\rho$ and Lamé coefficients, $\lambda$ and $\mu$;

2. mass density $\rho$ and the $\mathrm{P}$ wave and $\mathrm{S}$ wave velocities, $\alpha$ and $\beta$;

3. mass density $\rho$ and the $\mathrm{P}$ wave and $\mathrm{S}$ wave impedances.

Although the choice of parameters does not affect forward wave propagation simulations, it can have a profound effect on the inversion efficiency. The reciprocal relationship of each parameterization will be verified because each choice has different criteria for the reciprocity.

\section{Subtask 5.2: Chose robust objective function}

The purpose of seismic waveform inversion is to find the best model (i.e. subsurface distribution of properties) whose seismic responses are close to the observed data. The objective function is a measure of the difference between the observed and model-predicted seismic response. Because of the nonlinear nature of the problem, the choice of objective function can strongly affect the efficiency of the inversion process and the quality of the results. In our information theory formulation, the objective function changes the form of the probability distribution for the seismic image. We will seek the most sensitive objective function to quantify the mismatch between the synthetic and observed seismic response. In a 
sense, the best choice of the objective function is a reflection of our understanding and experience in arriving at the highest quality results and is therefore further information that increases the accuracy of our predictions.

\section{Subtask 5.3: Delineate seismic data protocols and gather data from industry partner} As our SEFD procedure requires a careful balance of geophone spacing, initial signal characteristics and other factors, we will work closely with industry partners to insure that the data is appropriate. This activity will also be a first step in writing the user's manual. Other considerations are (1) the target area of the wells and seismic data should be matched. Thus, the information from the wells should be applicable to seismic interpretation; and (2) the full description of real seismic data are required to make comparable synthetic data with real data such as acquisition geometry, seismic source property, and elevation information of the exploration area.

\section{Task 5.4: Demonstrate SEFD fracture prediction}

The main goal of this project is to identify statistical properties of fracture networks (such as volume fraction, number density, orientation distribution, and connectivity of fractures), we will use the relationships between seismic wave velocities and fracture network characteristics to process the results of our seismic inversion program to identify highpermeability fracture zones. This will be achieved in multiple steps.

Step 1 Core and well log data will be analyzed to estimate density, wave velocities, and fracture properties at the well.

Step 2 Using estimated properties from Step 1 as an initial model, our seismic inversion program will be executed to obtain the spatial distribution of density and seismic velocities.

Step 3 The properties of fracture networks between wells will be estimated by the relationships between the fracture characteristics and seismic properties which are obtained by the seismic inversion. We shall use a number of variables to characterize fracture networks (e.g. the statistical distribution of fracture density, orientation, length and aperture). Several approaches with varying degrees of complexity will be used and results compared to well logs and core data. 
Step 4 We shall then compare our predicted fracture network maps with observed ones and draw conclusions regarding the accuracies of our approach.

\section{Task 6 Outreach}

We believe that our massively parallel seismic inversion software is a significant advance in remote exploration. Due to the computational resources needed, our software can best be executed in supercomputing environments. In Task 4 we shall use the IBM SP and AVIDD systems available at Indiana University. A complication arises due to the large seismic data file sizes (hundreds of megabytes to gigabytes). We will modify the web interface we developed in Phase II, adjust for large file sizes, and make our seismic inversion software available to the research community. Due to the CPU requirements, the latter accessibility will be best achieved by installing our software at a DOE supercomputing site. We may also need to carry out selected simulations at this site during the course of the project. We shall provide a user's manual with detailed instructions and seismic data protocols required to achieve a reliable inversion via our methodology, and similarly for well log and other input data. We shall demonstrate our software to industry partners to obtain their feedback for inclusion in the user's manual and our final report. We will write the final report and present the results to DOE. 


\section{Literature}

Abriola, L.M., and K. Rathfelder. 1993. Mass balance errors in modeling two-phase immiscible flows: causes and remedies. Advances in Porous Media, 16: 223-239.

Aki, K. and Richards, P. G., 1980, Quantitative Seismology. Vol. 1. Theory and Methods: W. H. Freem Co.

Arntsen, B. and Carcione, J. M., 2001, Numerical simulation of the Biot slow wave in water-saturated Nivelsteiner sandstone, Geophysics, 66, 890-896.

Bachrach, R. and Nur, A., 1998, High-resolution shallow-seismic experiments in sand, Part I: Water table, fluid flow, and saturation, Geophysics, 63, 1225-1233.

Bathe, K.J. 1996. Finite element procedures. Englewood Cliffs, NJ: Prentice Hall.

Bathe, K.J., E. Ramm, and E.L. Wilson. 1975. Finite element formulations for large deformation dynamic analysis. International Journal for Numerical Methods in Engineering. 9:353-386.

Bradford, J. H., 2002, Depth characterization of shallow aquifers with seismic reflection, Part I-The failure of NMO velocity analysis and quantitative error prediction, Geophysics, 67, 89-97.

Brötz, R., Marschall, R., and Knecht, M., 1987, Signal adjustment of Vibroseis and impulsive source data, Geophy. Prosp., 35, 739-766.

Berenger, J. P., 1994, A perfectly matched layer for the absorption of electromagnetic waves, Journal of Computational Physics, 114, 185-200.

Biot, M. A., 1962, Mechanics deformation and acoustic propagation in porous media, Journal of Applied Physics, 33, 1482-1498.

Bookout, J. F., 1980, Devonian Shales: Unconventional gas sources, v. III, National Petroleum Council Committee on Unconventional Gas Sources.

Chew, W. C., and Weedon, W. H., 1994, A 3-D perfectly matched medium from modified Maxwell's equations with stretched coordinates, Micro. Opt. Tech. Lett., 7. 599-604.

Chew, W. C. and Jin, J. M., 1996, Perfectly matched layers in the discretized space: An analysis and optimization, Electromagnetics, 60, 325-340.

Chew, W. C. and Liu, Q. H., 1996, Perfectly matched layers for elastodynamics: A new absorbing boundary condition, Journal of Computational Acoustics, 4, 72-79. 
Clayton, R. and Engquist, B., 1977, Absorbing boundary conditions for acoustic and elastic wave equations, Bulletin of the Seismological Society of America, 67, 1529-1540.

Collett, J., 1879, Geological report on Harrison and Crawford Counties, Indiana, Indiana Geological Survey Annual Reports 8, 9, and 10, pp. 291-522.

Crase, E., Pica, A., Noble, M., McDonald, J. and Tarantola, A., 1990, Robust elastic nonlinear waveform inversion: Application to real data, Geophysics, 55, 527-538.

Crase, E., Wideman, C., Noble, M. and Tarantola, A., 1992, Nonlinear elastic waveform inversion of land seismic reflection data, 1992, J. Geohpys. Res., 97, 4685-4703.

Dai, N., Vafidis, A., and Kanasewich, E. R., 1995, Wave propagation in heterogeneous, porous media: A velocity-stress, finite-difference method, Geophysics, 60, 327-340.

Duta, N. C. and Odé, H., 1983, Seismic reflections from a gas-water contact, Geophysics, 48, 148-162.

Eisner, L. and Clayton, R. W., 2001, A reciprocity method for multiple-source simulations, Bull. Seism. Soc. Am., 91, 553-560.

Gauthier, O., Virieux, J. and Tarantola, A., 1986, Two-dimensional nonlinear inversion of seismic waveforms: Numerical results, Geophysics, 51, 1387-1403.

Graves, R. W., 1996, Simulating seismic wave propagation in 3D elastic media using staggered-grid finite differences, Bulletin of the Seismological Society of America, $86,1091-1106$.

Hicks, G. J. and Pratt, R. G., 2001, Reflection waveform inversion using local descent methods: Estimating attenuation and velocity over a gas-sand deposit, Geophysics, 66, 598-612.

Huyakorn, P.S., S. Panday, and Y.S. Wu. 1994. A three dimensional multiphase flow model for assessing NAPL contamination in porous and fractured media, I. Formulation. Journal of Contaminant Hydrology, 16: 109-130.

Jaynes, E.T. 1957. Information theory and statistical mechanics. Physical Review 106:620630.

Kalyoncu, R. S., Boyer, J. P., and Snyder, M. J., 1979, Characterization and analysis of Devonian shales as related to release of gaseous hydrocarbons: Well R-109, Washington County, Ohio, United States Department of Energy, Fossil Energy Report No. ORO-5205-T3. 
Kapur, J.N. 1988. Maximum entropy models in science and engineering. John Wiley, New York.

Kneib, G. and Kerner, C., 1993, Accurate and efficient seismic modeling in random media, Geophysics, 58, 576-588.

Kristek, J., Moczo, P., and Archuleta, R. J., 2002, Efficient methods to simulate planar free surface in the $3 \mathrm{D} 4^{\text {th }}$-order staggered-grid finite-difference schemes, Studia Geophysica et Geodaetica, 46, 355-381.

Kullback, S. 1959. Information theory and statistics. John Wiley, New York.

Lailly, P., 1983, The seismic inverse problem as a sequence of before stack migrations, in Bednar, J. B., Redner, R., Robinson, E. and Weglein, A., eds., Conference on Inverse Scattering: Theory and Application, Philadelphia, Soc. Ind. Apl. Math., 206220.

Lerche, I. 1991. Inversion of dynamical indicators in quantitative basin analysis models. I. Theoretical considerations. Mathematical Geology 23: 817-832.

Levander, A., 1988, Fourth-order finite-difference P-SV seismograms, Geophysics, 53, 1425-1436.

Levenberg, K., 1944, A method for the solution of certain nonlinear problems in least squares, Quart. Appl. Math., 2, 164-168.

Maubeuge, F., and I. Lerche. 1993. A north Indonesian basin: geo, thermal and hydrocarbon generation histories. Marine and Petroleum Geology 10:231-245.

Marquardt, D. W., 1963, An algorithm for least squares estimation of non-linear parameters, J. Soc. Industr. Appr. Math., 11, 431-441.

Mora, P., 1987, Nonlinear two-dimensional elastic inversion of multioffset seismic data, Geophysics, 52, 1211-1228.

Moczo, P., Kristek, J., Vavryuk, V., Archuleta, R. J., and Halada, L., 2002, 3D heterogeneous staggered-grid finite-difference modeling of seismic motion with volume harmonic and arithmetic averaging of elastic moduli and densities, Bulletin of the Seismological Society of America, 92, 3042-3066.

Ortoleva, P., ed. 1990. Self-organization in geological systems. Earth-Science Reviews 29(1-4)

Ortoleva, P. 1994. Geochemical self-organization. New York: Oxford University Press. 
Ortoleva, P., J.M. Maxwell, D. Payne, W. Sibo, and J. Comer. 1997. Naturally fractured reservoirs and compartments: A predictive basin modeling approach. In Fractured Reservoirs: Characterization and Modeling, edited by T.E. Hoak, A.L. Klawitter, and P.K. Blomquist. Rock Mountain Association of Geologists Guidebook, 67-102. Denver: RMAG.

Ortoleva, P. 1998. Basin compartment fundamentals, Topical Report (Project No. GRI97/0097). Chicago: Gas Research Institute.

Özdenvar, T. and McMechan, G. A., 1997, Algorithms for staggered-grid computations for poroelastic, elastic, acoustic, and scalar wave equations, Geophysical Prospecting, 45, 403-420.

Pratt, R. G., Shin, C. and Hicks, G. J., 1998, Gauss-Newton and full Newton methods in frequency-space seismic waveform inversion, Geophys. J. Int., 133, 341-362.

Rickard, Y. S., Georgieva, N. K., and Huang, W.-P., 2003, Application and optimization of PML ABC for the 3-D wave equation in the time domain, IEEE Transactions on Antennas and Propagation, 51, 286-295.

Pride, S. R., Tromeur, E., and Berryman, J. G., 2002, Biot slow-wave effects in stratified rock, Geophysics, 67, 271-281.

Rodi, W. L., 1976, A technique for improving the accuracy of finite element solutions for magnetotelluric data, Geophys. J. R. astr. Soc., 44, 483-506.

Sasaki, Y., 1989, Two-dimensional joint inversion of magnetotelluric and dipole-dipole resistivity data, Geophysics, 54, 254-262.

Sheen, D.-H., Tuncay, K., Baag, C.-E. and Ortoleva, P. J., 2004, A velocity-stress staggered-grid finite-difference method with perfectly matched layers for 3-D poroelastic wave propagation, Geophysics, (submitted).

Shin, C., Yoon, K., Marfurt, K. J., Park, K., Yang, D., Lim, H. Y., Chung, S. and Shin, S., 2001, Efficient calculation of a partial-derivative wavefield using reciprocity for seismic imaging and inversion, Geophysics, 66, 1856-1863.

Sorgenfrei, H., Jr., 1952, Gas production from the New Albany Shale: M.S. thesis, Indiana University, Bloomington.

Tarantola, A., 1984, Inversion of seismic reflection data in the acoustic approximation, Geophysics: 49, 1259-1266. 
Tarantola, A., 1987, Inverse problem theory: Methods for data fitting and parameter estimations: Elsevier Science Publ. Co., Inc.

Tarantola, A. and Valette, B., 1982, Generalized non linear inverse problems solved using the least-squares criterion, Rev. of Geophys. and Space Physics, 20, 219-232.

Tuncay, K. and Corapcioglu, M. Y., 1996, Body waves in poroelastic media saturated by two immiscible fluids, Journal of Geophysical Research, 101, 25149-25159.

Tuncay, K. Park, A, and Ortoleva P. (2000a). Sedimentary basin deformation: An incremental stress rheology approach. Tectonophysics, Vol. 323, 77-104.

Tuncay, K. Park, A, and Ortoleva P. (2000b). A forward model of three dimensional fracture orientation and characteristics._Journal of Geophysical Research Vol. 105, 16719-16735.

Tuncay, K., and P. Ortoleva. 2001. Salt tectonics as a self-organizing process: A three dimensional reaction, transport and mechanics model. Journal oGeophysical Research 106: 803-818.

Tuncay, K., A. Khalil, and P. Ortoleva. 2001. Failure, memory and cyclic fault movement. Bulletin of Seismological Society of America 91: 538-552.

Tuncay, K. and P. Ortoleva. 2002. Probability functionals, homogenization and comprehensive reservoir simulators. Resource Recovery, Confinement, and Remediation of Environmental Hazards, Institute of Mathematics and its Applications volume 131, Editors: J. Chadam, A. Cunningham, R. E. Ewing, P. Ortoleva, and M. F. Wheeler, Springer-Verlag, New York, 161-178.

Yu, Z., and I. Lerche, and Q. Bour. 1995, Inversion of dynamical indicators in quantitative basin analysis models. III. Multiwell information and two-dimensional case histories. Mathematical Geology 27: 41-68.

Woodbury, A.D., and T.L. Ulrych. 1996. Minimum relative entropy inversion: theory and application to recovering the release history of a groundwater contaminant. Water Resources Research 32: 2671-2681.

Zielinski, R. E., and Moteff, J. D., 1980, Physical and chemical characterization of Devonian gas shale: Quarterly Status Report (April 1-June 30, 1980), Miamisburg, Ohio, Monsanto Research Corporation, Mound Facility, MLM-EGSP-TPR-Q-014. 
Zeng. Y. Q., He, J. Q., and Liu, Q. H., 2001, The application of the perfectly matched layer in numerical modeling of wave propagation in poroelastic media, Geophysics, 66, 1258-1266.

Zeng, Y. Q. and Liu, Q. H., 2001, A staggered-grid finite-difference method with perfectly matched layers for poroelastic wave equations, Journal of the Acoustical Society of America, 109, 2571 -2580.

Zhao, K., and I. Lerche. 1993. Inversion of dynamical indicators in quantitative basin analysis models. II. Synthetic tests and a case history using dynamical indicator tomography. Mathematical Geology 25: 107-123.

Zhu, X. and McMechan, G. A., 1991, Numerical simulation of seismic responses of poroelastic reservoirs using Biot theory, Geophysics, 56, 328-339.

Ziolkowski, A., 1991, Why don’t we measure seismic signatures?, Geophysics, 56, 190-201. 


\section{Appendix A}

Tandon, K., K. Tuncay, J. Comer, K. Hubbard, and P. Ortoleva. 2004. Estimating tectonic parameters for sedimentary basin evolution: Integration of basin modeling and reflection seismology, Geophysical Journal International, Vol 156, 129-139. 


\title{
Estimating tectonic history through basin simulation-enhanced seismic inversion: geoinfomatics for sedimentary basins
}

\author{
Kush Tandon, ${ }^{1, *}, \dagger$ Kagan Tuncay, ${ }^{1}$ Kyle Hubbard, ${ }^{1}$ John Comer $^{2}$ and Peter Ortoleva ${ }^{1}$ \\ ${ }^{1}$ Laboratory for Computational Geodynamics, Department of Chemistry, Indiana University, Bloomington, IN 47405-7102, USA. \\ E-mails: ktuncay@indiana.edu; ortoleva@indiana.edu \\ ${ }^{2}$ Indiana Geological Survey, 611 North Walnut Grove, Bloomington, IN 47405-2208,USA
}

Accepted 2003 September 10. Received 2003 September 1; in original form 2002 December 3

\begin{abstract}
S U M M A R Y
A data assimilation approach is demonstrated whereby seismic inversion is both automated and enhanced using a comprehensive numerical sedimentary basin simulator to study the physics and chemistry of sedimentary basin processes in response to geothermal gradient in much greater detail than previously attempted. The approach not only reduces costs by integrating the basin analysis and seismic inversion activities to understand the sedimentary basin evolution with respect to geodynamic parameters - but the technique also has the potential for serving as a geoinfomatics platform for understanding various physical and chemical processes operating at different scales within a sedimentary basin.

Tectonic history has a first-order effect on the physical and chemical processes that govern the evolution of sedimentary basins. We demonstrate how such tectonic parameters may be estimated by minimizing the difference between observed seismic reflection data and synthetic ones constructed from the output of a reaction, transport, mechanical (RTM) basin model. We demonstrate the method by reconstructing the geothermal gradient. As thermal history strongly affects the rate of RTM processes operating in a sedimentary basin, variations in geothermal gradient history alter the present-day fluid pressure, effective stress, porosity, fracture statistics and hydrocarbon distribution. All these properties, in turn, affect the mechanical wave velocity and sediment density profiles for a sedimentary basin. The present-day state of the sedimentary basin is imaged by reflection seismology data to a high degree of resolution, but it does not give any indication of the processes that contributed to the evolution of the basin or causes for heterogeneities within the basin that are being imaged. Using texture and fluid properties predicted by our Basin RTM simulator, we generate synthetic seismograms. Linear correlation using power spectra as an error measure and an efficient quadratic optimization technique are found to be most effective in determining the optimal value of the tectonic parameters. Preliminary 1-D studies indicate that one can determine the geothermal gradient even in the presence of observation and numerical uncertainties. The algorithm succeeds even when the synthetic data has detailed information only in a limited depth interval and has a different dominant frequency in the synthetic and observed seismograms. The methodology presented here even works when the basin input data contains only 75 per cent of the stratigraphic layering information compared with the actual basin in a limited depth interval.
\end{abstract}

Key words: complex systems, data assimilation, geoinfomatics, geothermal gradient, sedimentary basin modelling.

\footnotetext{
*Present address: College of Oceanic and Atmospheric Sciences, Oregon State University, Corvallis, OR 97331-5503. E-mail: kush@coas.oregonstate.edu

${ }^{\dagger}$ To whom the correspondence should be addressed.
}

\section{INTRODUCTION}

One of the challenges for understanding a complex system that evolves over millions of years, such as a sedimentary basin is the very poor constraint on parameters that govern or significantly affect the physical and chemical processes operating in a sedimentary basin. In a typical approach studying the physics and chemistry 
involved in the formation of sedimentary basins either in an academic or industrial setting, seismic inversion and basin modelling are essentially carried out as independent activities. Even when information from seismic inversion is used, it is only a small part of the basin modelling effort and it does not attempt to capture the richness and diversity of processes involved. However, a comprehensive basin reaction, transport, mechanical (RTM) numerical model makes predictions concerning fluid and rock property distributions that could, in principal be used to enhance the quality of seismic inversion. It is the objective of this study to integrate and automate the seismic inversion and basin modelling efforts into a cost-effective technology that enhances the quality and general information content of both. Also, one of the fundamental problems in performing inversion in earth sciences is the lack of enough detailed information concerning the Earth to make predictions that can be tested with confidence using various data sets. Therefore, it is an important issue to lay down the theoretical framework for methodology for detailed predictions for the evolution of the outermost veneer of the Earth's crust, the sedimentary basins, and approaches for basin data assimilation.

Evolution of a sedimentary basin depends on the strong coupling among many processes (hydrocarbon generation, fracturing, compaction, etc.). Tectonic parameters (such as geothermal gradient and extension/compression histories) strongly affect these physical and chemical processes. Uncertainties in the input parameters needed to run a basin model, therefore, lead to uncertainties in the predictions. Here, a method is presented to constrain these uncertain tectonic parameters to arrive at a higher resolution geophysical imaging tool by integrating reflection seismology data and RTM basin modelling. In Fig. 1, we suggest how this approach can be automated in a highly parallelizable, automated computational approach.

Reflection seismic data are commonly used to delineate the sedimentary basin architecture, structural evolution and sedimentary process as it is a very high-resolution, geophysical subsurface imaging technique (McQuillin et al. 1984). However, seismic reflection data alone cannot discriminate amongst many factors that cause the acoustic impedance contrast (e.g. gas saturation, finely laminated sediments and gouge) imaged by reflection seismology. A limitation of many conventional geophysical techniques is that they are not robust and are fraught with subjective interpretation.

Seismic inversion is often used to determine rock and fluid properties (composition, fracture intensity and orientation, fluid saturation and overpressure). Most seismic inversion techniques limit their analysis to a small set of variables to allow invertibility of the seismic reflection data. For example, Mallick et al. (1998) used the amplitude variation with offset technique to predict fracture orientations. Ramos \& Davis (1997) studied the detection of fracture density variations by delineating zones of large Poisson ratio contrasts. These and other studies (Cabrera 1996; Boadu 1998) show that predictions using reflection seismology are limited to a few rock properties. We suggest that seismic interpretation and our understanding of sedimentary basins can be greatly enhanced through the integration of comprehensive basin modelling (Tuncay et al. 2000a,b; Tuncay \& Ortoleva 2001, 2003) and reflection seismic data. We have no knowledge of a previous attempt to study the complex evolution of heterogeneities that act as acoustic impedance contrasts and the interaction of tectonics from a comprehensive basin modelling approach. Our objective is to develop a technology that will enable us to estimate a set of basin parameters constrained by the observed reflection seismic data. This could allow us to aid the remote detection of economically impor-

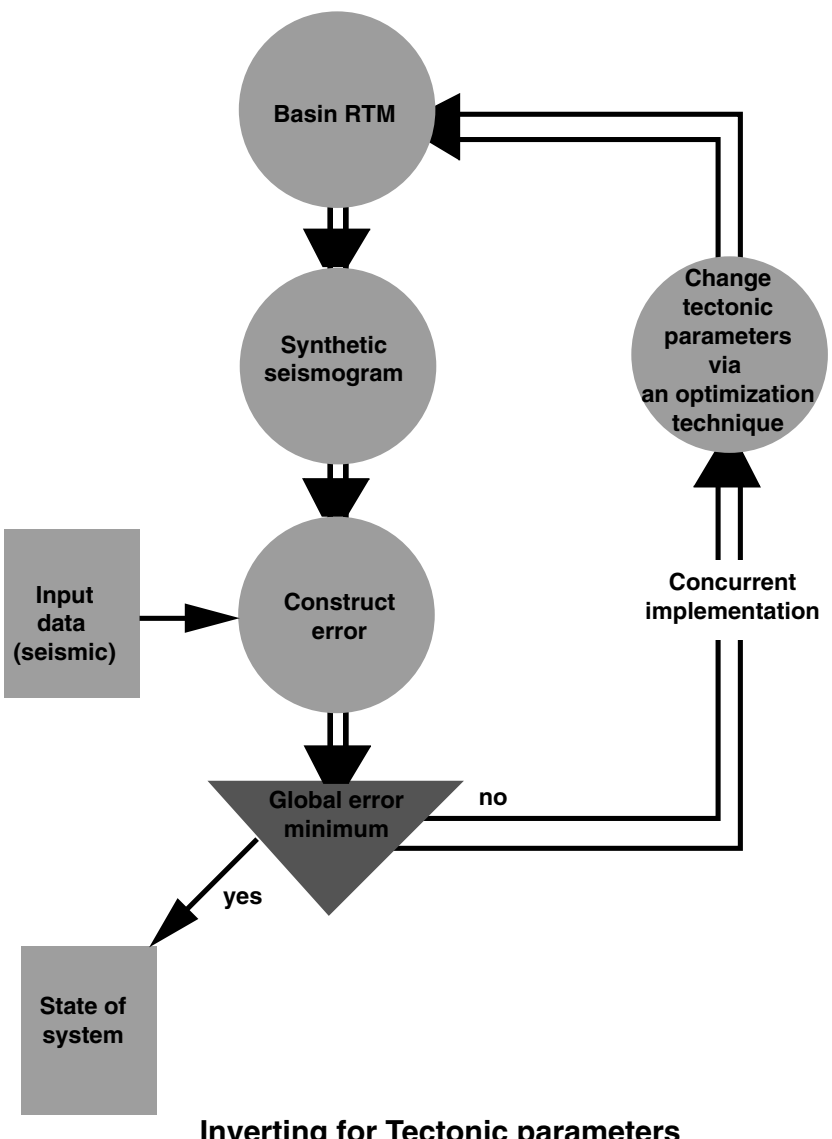

Inverting for Tectonic parameters

Figure 1. A flow chart for estimating the tectonic parameters automatically through our seismic inversion-basin modelling algorithm. This technique has been implemented in a parallel fashion through a common error database, as shown.

tant fractured compartments and conventional reservoirs as well as the estimation of tectonic conditions to which the basin was subjected.

As a demonstration of our technique, the geothermal gradient is estimated using a comprehensive simulator, Basin RTM (Tuncay et al. 2000a,b; Tuncay \& Ortoleva 2001, 2003) and reflection seismic data. In this study we use a Basin RTM simulated synthetic seismogram at $30{ }^{\circ} \mathrm{C} \mathrm{km}^{-1}$ geothermal gradient as the 'observed' data to evaluate the vulnerability of the approach to noise and incomplete data (Fig. 2). The following issues are investigated.

(1) The choice of error measure and its robustness to noisy observed data.

(2) The performance of the technique when a complete, detailed description of the stratigraphy is only available within a limited interval of the rock column. Usually over all the basins in the world, only a limited part of the sedimentary column in that basin is very well studied and understood so that it can be used as an input to a comprehensive basin simulator with confidence.

(3) The effect of the difference of the dominant frequency between observed and synthetic data.

(4) The influence of missed lithologies in the sediment column used as input for the basin simulator. Any input to a basin modelling effort will always be fraught with uncertainties and missed lithologies. 


\section{"Made-up" rock column using Basin RTM}

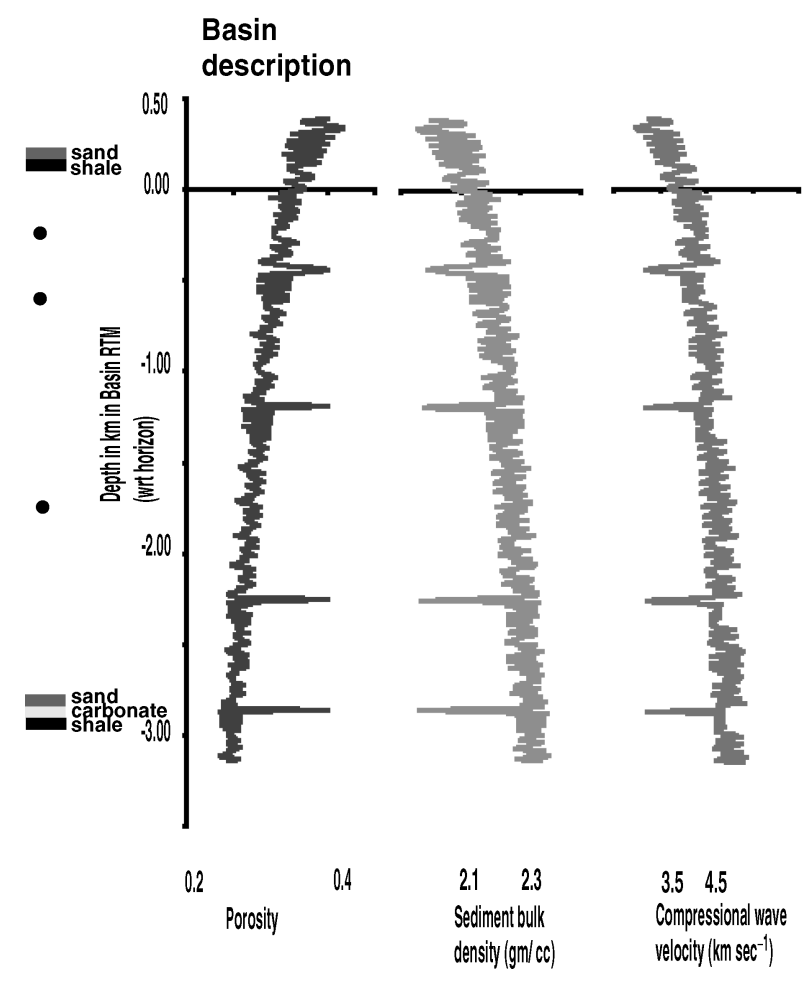

Figure 2. A synthetic profile of porosity, sediment bulk density and $P$-wave velocity generated by a Basin RTM simulation for a geothermal gradient of $30{ }^{\circ} \mathrm{C} \mathrm{km}^{-1}$ with more than $3 \mathrm{~km}$ thick basin interbedded with shales, sandstone and carbonates. These profiles are pseudo-well logs made through complex interaction of various reaction, transport, mechanical processes defined in Section 2 via Basin RTM. The $30{ }^{\circ} \mathrm{C} \mathrm{km}^{-1}$ simulation is used as 'observed' data for the experiments conducted in this study. The use of parentheses is to distinguish our discussion pertaining to real data sets.

\section{APPROACH}

\subsection{Comprehensive basin modelling}

In order to generate the synthetic response, a comprehensive RTM model is required. The advantage of a comprehensive RTM basin model is not only its potential for reliable predictions, but also its ability to predict a fuller suite of the parameters needed to calculate the synthetic response to be compared with a variety of observed data types (for example, seismic and well logs). Predictions include the pressure and composition of the various pore fluid phases; the shape, size, packing and abundance of the minerals; fracture network statistics; and in situ stress. These rock and fluid parameters can help one estimate oil and gas reserves in place and the hydrologic and mechanical properties of reservoirs and other sedimentary units. There are two types of quantitative physico-chemical basin models presently in use.

(1) Conventional basin models that have the capability to simulate multiphase flow, but use empirical laws for compaction (Ungerer et al. 1990; Forbes et al. 1992; Person \& Garven 1992; Maubeuge \& Lerche 1993, 1994; Bour \& Lerche 1994; Luo \& Vasseur 1995, 1996; Person et al. 1995; Roberts \& Nunn 1995; Wieck et al. 1995; Yu et al. 1995; Gordon \& Flemings 1998; Wang \& Xie 1998; McPherson \& Garven 1999; McPherson \& Bredehoeft 2001). Some of these models include petroleum generation (Ungerer et al. 1990;
Maubeuge \& Lerche 1993, 1994; Luo \& Vasseur 1996). Fracturing, however, which is an important factor in tight reservoirs, is only considered by a few research groups (Maubeuge \& Lerche 1993, 1994; Roberts \& Nunn 1995; Wang \& Xie 1998; McPherson \& Bredehoeft 2001) and is accounted for by assuming that rocks fracture when pore pressure exceeds a certain fraction of the overburden stress. This assumption essentially eliminates the dependence of fracturing on lithologic properties, a fact that is in contradiction to observations from sedimentary basins all over the world. In other approaches, empirical laws are used to relate porosity to effective stress, temperature and other variables to model compaction driven flow in sedimentary basins; this assumption also ignores the dependence of rock properties on lithology.

(2) Basin models with a stress/deformation module (Schneider et al. 1996; Luo et al. 1998; Suetnova \& Vasseur 2000): these models have a better accounting of stress and deformation evolution. However, they ignore multiphase flow and petroleum generation processes that significantly affect the stress and the deformation of a sedimentary basin.

To capture the essence of coupled processes operating in sedimentary basins, a numerical basin model should at least include the following:

(1) A deformation model that accounts for poroelasticity and irreversible deformation mechanisms such as pressure solution and fracturing.

(2) A fracture network dynamics model that is capable of being extended to 3-D.

(3) Rheologic and multiphase parameters co-evolved with diagenesis, compaction and fracturing.

(4) Multiphase flow and petroleum generation.

(5) Inorganic fluid and mineral reactions.

(6) Heat transfer.

(7) Reconstruction of sedimentation/erosion history.

Recently, Tuncay et al. (2000a) developed an incremental stress rheology approach for sedimentary basins, which integrates many types of processes that affect rock properties (including, for example, poroelasticity, non-linear viscosity and pressure solution). The statistical treatment of fracture network dynamics provides a significant improvement over existing basin models (Tuncay et al. $2000 \mathrm{~b}$ ) as it allows the quantification of anisotropy created dynamically by fracturing and its effects on the total rate of strain and rock mechanical and fluid transport properties. Rocks fracture due to the difference between the fluid pressure and the least compressive stress. However, as fractures open, overall rock volume increases and fluid pressure in the fractures compresses the rock, increasing the compressive stress normal to the fracture plane. Thus, fracturing is a self-limiting process: first, as fractures open, they provide a pathway for fluid escape/depressuring and secondly, the volumetric strain caused by fractures increases the confining stress that reduces the rate of fracture growth. Payne et al. (2000) and Tuncay \& Ortoleva (2001) have applied this comprehensive approach to the Piceance basin (1-D) and in salt tectonic regimes (2-D).

In this study, we account for fracture network dynamics; incremental stress rheology (with poroelasticity and irreversible temperature-dependent viscous deformation) and single-phase fluid flow for a 1-D approach. The input for Basin RTM is mostly from published literature, public domain data, well logs, etc., and sometimes site specific oil industry propriety data. The input for Basin RTM includes sedimentation composition and grain size, sedimentation/erosion rate, subsidence rate, sea level history, and a 


\section{Synthetic seismograms (using 80 Hz Ricker wavelet)}

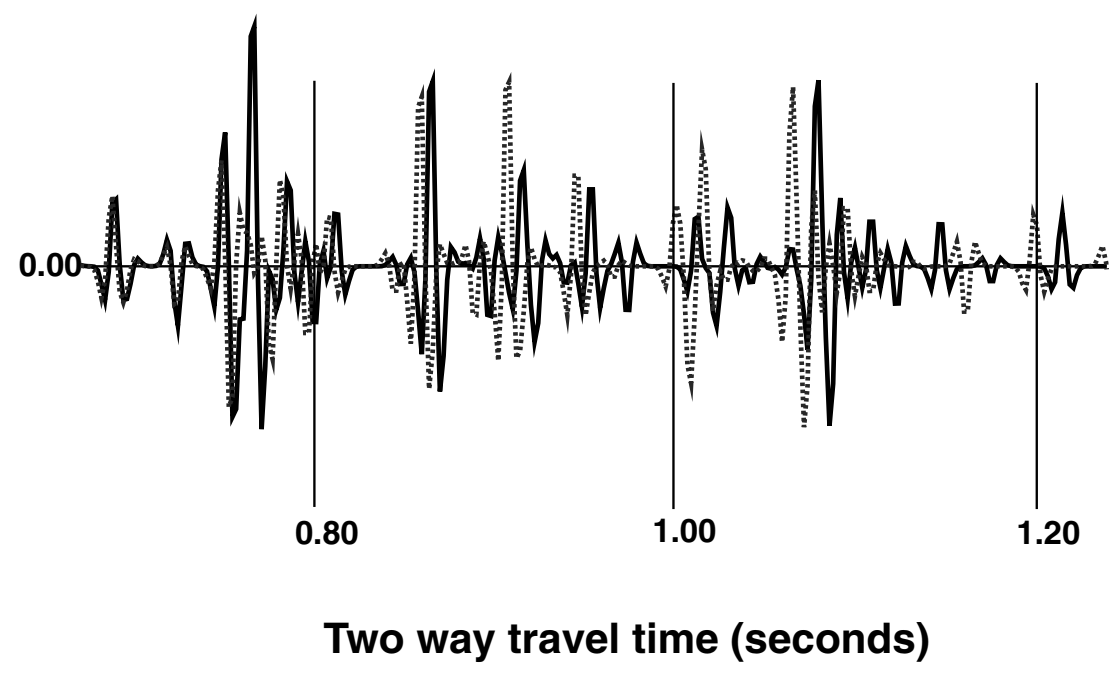

\section{- generated by basin RTM @ dT/ dZ = 30 degree $\mathrm{C} \mathrm{km}^{-1}$ generated by basin RTM @ dT/ dZ = 45 degree $\mathrm{C} \mathrm{km}^{-1}$}

Figure 3. A comparison of synthetic seismograms generated from Basin RTM output at 30 and $45^{\circ} \mathrm{C} \mathrm{km}^{-1}$ illustrates the strong dependence of the seismic signal on the tectonic history. This interesting observation caused by temperature dependence for fluid and rock properties underlies the viability of the approach suggested in Fig. 1.

number of phenomenological parameters. This all evaluates the rock and fluid transport properties, such as permeability, bulk and shear viscosity (Tuncay et al. 2000a,b, 2001). Basin RTM also has access to thermodynamic and kinetic data for most water-rock interaction phenomena. For example, gamma logs are used to determine the percentage shaliness of a stratigraphic column (Schlumberger Log Interpretation 1972) to be one of the inputs to Basin RTM. We assign a standard mineralogical composition in terms of the percentage of clay minerals, quartz, feldspar, etc. for 100 per cent shales, sandstones and carbonates (Blatt \& Traey 1995). Using a percentage shaliness that we calculated from the logs, we calibrate the mineralogical composition accordingly for different rock units when we build our sedimentary basin for numerical simulation.

\subsection{Algorithm}

Although basin models require a large number of phenomenological parameters and geologic boundary conditions, only a few studies have focused on the utilization of observed data to constrain the model (Lerche 1991; Maubeuge \& Lerche 1993; Zhao \& Lerche 1993; Yu et al. 1995). Uncertainty in basin modelling is reviewed in detail by Tuncay \& Ortoleva (2003). Here we concentrate on data/model integration to determine tectonic parameters, notably the temperature gradient, using reflection seismic data.

Our approach for automatically estimating the geothermal gradient based on our seismic inversion-basin modelling algorithm is as follows. Fig. 1, (1) an optimization technique (iterative quadratic fitting or simulated annealing) is used to select a geothermal gradient for a Basin RTM simulation. (2) Using this geothermal gradient, the basin simulator predicts the present-day rock and fluid properties. (3) Predicted rock and fluid properties are used to construct sediment bulk density and $P$-wave velocity profiles (Fig. 2), (4) a synthetic seismogram is generated from the calculated properties (Fig. 3), (5) the error between the synthetic and observed seismograms is computed and used to guide the search for the error-minimizing geothermal gradient in an iterative fashion.

\subsection{Synthetic seismograms}

A linear convolution approach was implemented for computing the synthetic seismograms (Peterson et al. 1955). The reflection coefficients were calculated from the Basin RTM output and convolved with a source wavelet to produce the synthetic seismogram. The linear convolution approach does not take into account the effects of internal multiples, attenuation or spherical divergence. $P$-wave velocity depends on pore pressure, grain size, porosity, rock composition, fracture statistics, etc. (Murphy 1982; Klimentos \& McCann 1983; Nagano 1998). In this study, $P$-wave velocity was calculated as a function of the porosity and texture of the unfractured rock using the approach of Berryman (1986). Fig. 3 shows a comparison of two synthetic seismograms obtained for different geothermal gradients. In this particular example, higher geothermal gradient results in a more compact rock column as a result of higher rate of compaction. However, one should be cautious in generalizing this result. Higher temperatures can also increase fluid pressure to lithostatic levels and can retard/stop compaction as effective stress becomes small. Furthermore, as mineral composition of a sedimentary layer varies, compaction rates do not increase uniformly throughout the basin.

\subsection{Error measures}

Various measures of the error between the observed and synthetic seismograms were used to evaluate their suitability for our 
algorithm. Measures for the error in the time-series $x_{i}$ (observed) and $y_{i}$ (synthetic) are as follows.

Our definition for linear correlation is the same as Pearson's linear correlation coefficient, $r$ (Press et al. 1993), except for a minus sign:

$r=-\frac{\sum_{i=1}^{N}\left(x_{i}-\bar{x}\right)\left(y_{i}-\bar{y}\right)}{\sqrt{\sum_{i=1}^{N}\left(x_{i}-\bar{x}\right)^{2}} \sqrt{\sum_{i=1}^{N}\left(y_{i}-\bar{y}\right)^{2}}}$,

where $\bar{x}=\frac{1}{N} \sum_{j=1}^{N} x_{j}$ and similarly for $\bar{y}$. A minus sign is incorporated so that a minimum in the error occurs at the observed geothermal gradient. According to the above formulation, a 'perfect correlation' between two time-series is -1 . If the values of $r$ are close to zero, then the two time-series $x_{i}$ and $y_{i}$ are uncorrelated. Alternatively, power spectra for the time-series $x_{i}$ and $y_{i}$ can be used to quantify error.

The mean square error $e$ for time-series $x_{i}$ and $y_{i}$ is defined via

$e=\sum_{i=1}^{N}\left(x_{i}-y_{i}\right)^{2}$.

The mean square error $e_{\mathrm{na}}$ based on next same arrival time is defined via

$e_{\mathrm{na}}=\sum_{i=2}^{N}\left(t_{i}^{x}-t_{i}^{y}\right)^{2}$,

where $t_{i}^{x}=x_{i}-x_{i-1}$ and similarly for $t^{y}{ }_{i}$.

\subsection{Optimization techniques}

We experimented with two optimization techniques (iterative quadratic fit and simulated annealing) for finding the global minimum of the various error measures. A critique of Monte Carlo, simulated annealing, and genetic algorithms as applied to minimization methods in geophysics is presented in Sen \& Stoffa (1995). As greedy algorithms, such as simulated annealing require many iterations and as each iteration requires a computationally expensive basin simulation in the present context, one expects that they would not be practical. In the iterative quadratic fit method, we fit the error to a quadratic function based on simulations for three geothermal gradients (or $3^{d}$ simulations when the number of tectonic parameters to be estimated is $d$ ). Using the coefficients from the curve fit, the geothermal gradient that minimizes the error is computed. The location of this minimum is then used as the centre point for the next quadratic fitting cycle to refine the location of the minimum. This cycle is repeated until the error difference between the minima from most recent and previous iteration is less than a specified tolerance. The weakness of this method is that it may only find a local, and not a global, minimum.

Simulated annealing is a minimization algorithm capable of finding a global minimum despite the presence of local minima (Otten \& van Ginneken 1989; Sen \& Stoffa 1995). The simulated annealing technique implemented here is based on the Cauchy cooling algorithm (Szu \& Hartley 1987, Taygeta Scientific Inc.). The Cauchy cooling algorithm is a fast annealing technique compared to the other Boltzmann algorithms (Ingber 1993). Rothman (1985, 1986), Basu \& Frazer (1990) and Sen \& Stoffa (1991) have used simulated annealing in geophysical applications. The simulated annealing approach is quite analogous to the manner in which liquids freeze or metals crystallize during annealing (Kirkpatrick et al. 1983). The simulated annealing search initially is very disorderly similar to the thermodynamic state of the melt at high temperatures (Kirkpatrick et al. 1983). Such an approach enables one to span the entire error space and not be stuck in local minima during optimization. As error minimization continues, the approach becomes more orderly and enables one to find a global minimum in a similar fashion as a system in nature achieves thermodynamic equilibrium when cooled slowly (Kirkpatrick et al. 1983). The algorithm is set up in terms of a thermodynamic system described in terms of temperature, energy, and with the probability of accepting a change in energy given through Boltzmann factor (Kirkpatrick et al. 1983). Our experience showed that the performance of simulated annealing optimatization technique depended on the rate with which the system was cooled, meaning thereby how quickly we proceeded in our choice of geothermal gradients (coarser sampling of the error space) toward the global minimum. A slow cooling approach was expensive but was much more robust in finding a global minimum compared with a faster approach. The strength of simulated annealing is that the algorithm is capable of determining a global minimum even when the error function is very rugged in shape, full of local minima. However, the drawback of the simulated annealing algorithm is that it is a greedy algorithm and quite time-consuming computationally.

\section{NUMERICAL EXPERIMENTS}

Numerical experiments are carried out using a Basin RTM simulation run with a geothermal gradient of $30{ }^{\circ} \mathrm{C} \mathrm{km}^{-1}$ to generate the 'observed' seismic data. As the present study concentrates on the development and the robustness of the technique, we used a seismogram from a $30{ }^{\circ} \mathrm{C} \mathrm{km}^{-1}$ basin simulation as a proxy for 'observed' data rather than using actual reflection seismic data. In an approach such as this, an actual reflection seismic data will be a single trace taken from a multichannel, stacked, migrated, reflection seismic section intersecting a well or borehole location, as used in many standard seismogram modelling approaches (Lorenzo \& Hesselbo 1996; Tandon et al. 1998).

For our study, we built a $3.5 \mathrm{~km}$ sedimentary basin that is a composite made from data originally from Piceance and East Texas basins. The results of Fig. 2 are generated from a 112 myr simulation of a $3.5 \mathrm{~km}$ (present-day) stratigraphic column with interbedded sand, shale and carbonate lithologies. The 'observed' rock record consists of 225 layers, with thicknesses varying from 5 to $15 \mathrm{~m}$. The corresponding seismogram and the associated data used in Basin RTM from $30{ }^{\circ} \mathrm{C} \mathrm{km}^{-1}$ simulation is the 'observed' data for our set of experiments. Different levels of random noise are added to this 'observed' seismogram (see the Appendix). Seismograms from Basin RTM simulations at different geothermal gradients are part of the inversion technique (Fig. 1) to figure out the correct geothermal gradient via error minimization.

We assume that the 'observed' seismic reflection data contains ambient noise that is coloured in nature (Ursin et al. 1996). The majority of the noise in an observed data is removed via higherfold stacking, f-k, and coherent filtering (Yilmaz 1987). Frequency content of ambient noise typical in seismic reflection data can be both low (0-2 Hz) and high (16-32 Hz) (Larner et al. 1983; Yilmaz 1987). However, in general, most noise in the stacked seismic data is source generated and scattered surface waves which dominantly have lower frequency content (Larry Brown, personal communication). The effect of incoherent noise is ignored since they are quite low in amplitude (Sheriff \& Geldart 1995).

Experimental studies in source-generated noise identify a frequency bandwidth of 2-12 Hz for noise analysis (Jolly \& Mifsud 1971). Similarly, experiments on surface waves display a $0.2-5 \mathrm{~Hz}$ bandwidth (Douze 1964). A seismic noise experiment at Roosevelt Hot Springs showed a bandwidth of $0.5-10 \mathrm{~Hz}$ and frequencies 
Power spectrum of the "observed" data
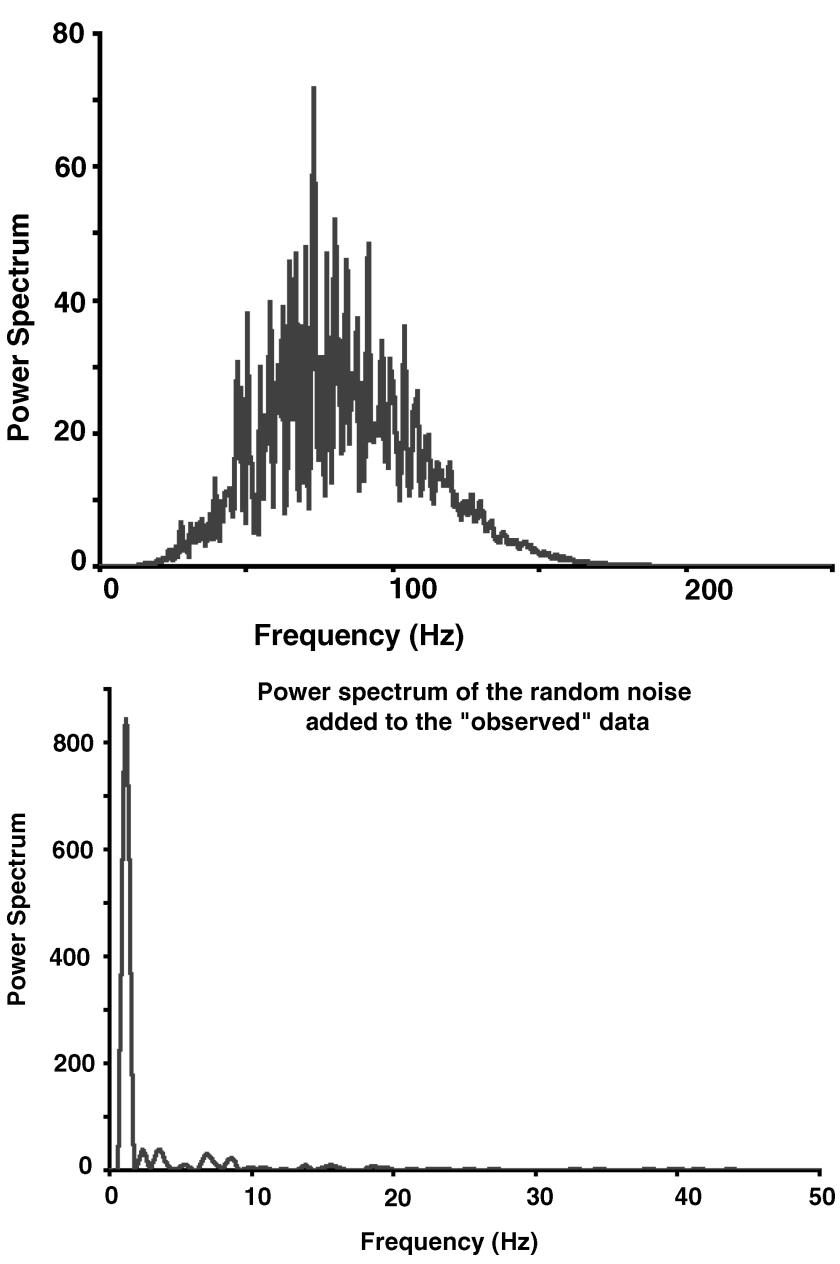

Figure 4. (a) A power spectrum of the 'observed' noise-free seismic data (Fig. 3). (b) A power spectrum of the noise added to the 'observed' data. The figure shows the noise at 50 per cent level and its dominantly low frequency content to simulate typical noise that could be present in a stacked seismic reflection data even after seismic data processing (Yilmaz 1987).

$>10 \mathrm{~Hz}$ displayed no spatial coherence (Douze \& Laster 1979). Therefore, in this study the bandwidth for the random noise that is added to the 'observed' data is $0.6-20 \mathrm{~Hz}$. The contribution from higher frequencies is generally considered unimportant for a stacked trace and was thus neglected (Fig. 4b). The frequency bandwidth of the 'observed' data (Fig. 4a) is much wider than that of the noise.

\section{Experiment 1: choice of best error measure}

Numerical experiments (Figs 5-7) show that linear correlation and quadratic error yield a single minimum in the error over the range of geothermal gradient values $\left(15-45^{\circ} \mathrm{C} \mathrm{km}^{-1}\right)$ typically found in sedimentary basins (Turcotte \& Schubert 1982). This character persists in the presence of noisy 'observed' data (Figs 5-7). The linear correlation error both using the time-series and their power spectra displays a global minimum even when the noise level in the 'observed' data is 75 per cent (Figs 5 and 6). If in the real reflection seismic data, higher-frequency noise was present then the global minimum will not be observable at 75 per cent noise level. In the quadratic error, the global minimum is not well defined since the presence of random noise flattens the error function at lower levels

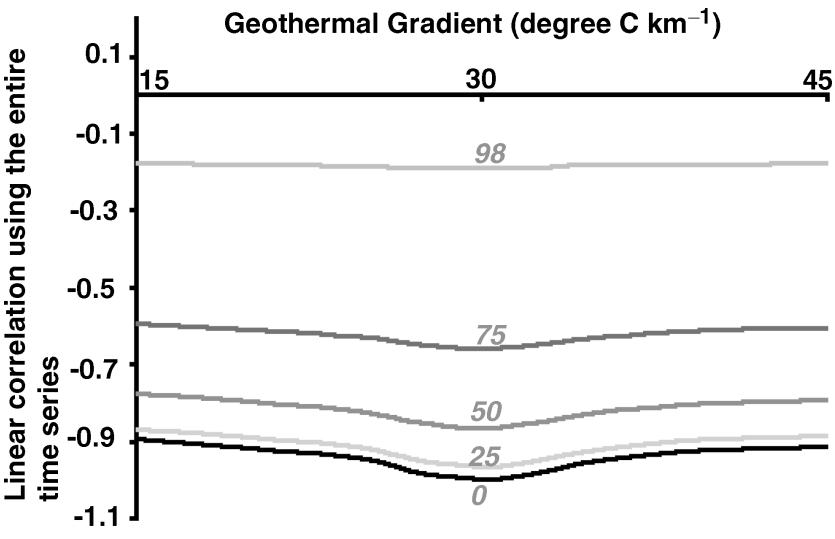

italic number next to a curve $=$ noise level in percentage

Figure 5. The variation of linear correlation error to be part of the seismic inversion-basin modelling scheme (Fig. 1) at different noise levels using the entire time-series.

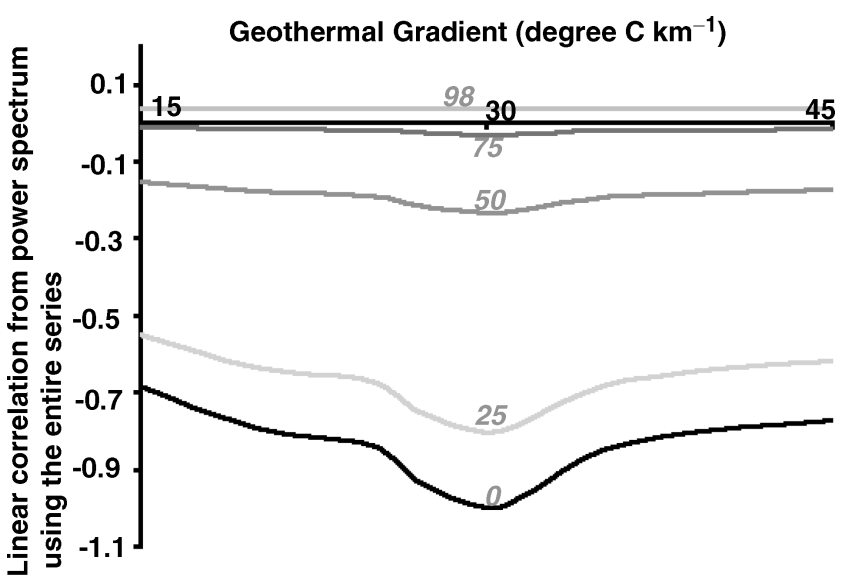

italic number next to a curve $=$ noise level in percentage

Figure 6. A linear correlation error based on the power spectrum of the seismograms to be part of the seismic inversion-basin modelling scheme (Fig. 1) at different noise levels.

than for linear correlation (Fig. 7). This implies the effect of attenuation and geometric spreading in the observed seismic data can make any error measure based on mean square amplitude difference unreliable. Fig. 8 shows that the quadratic error based on next arrival time does not display the global minimum at noise levels as low as 25 per cent. We conclude that linear correlation error using timeseries or power spectra was found to be the most effective measure for our methodology.

\section{Experiment 2: model data with detailed stratigraphic layering} in a limited depth interval

When using real basin data, it is very unlikely that one would have detailed information on lithologies over the entire stratigraphic column that can be used as input data in a comprehensive basin simulator and perform seismic inversion. We think this is an important experiment to test the feasibility of such an approach, as the entire stratigraphic column in any basin around the world is not very well understood and studied. To study the effects of the lack of stratigraphic data in the shallower depths, the first second out of $1.742 \mathrm{~s}$ two-way traveltime is truncated in the synthetic seismograms. With this truncation, only the last 42 per cent of the 


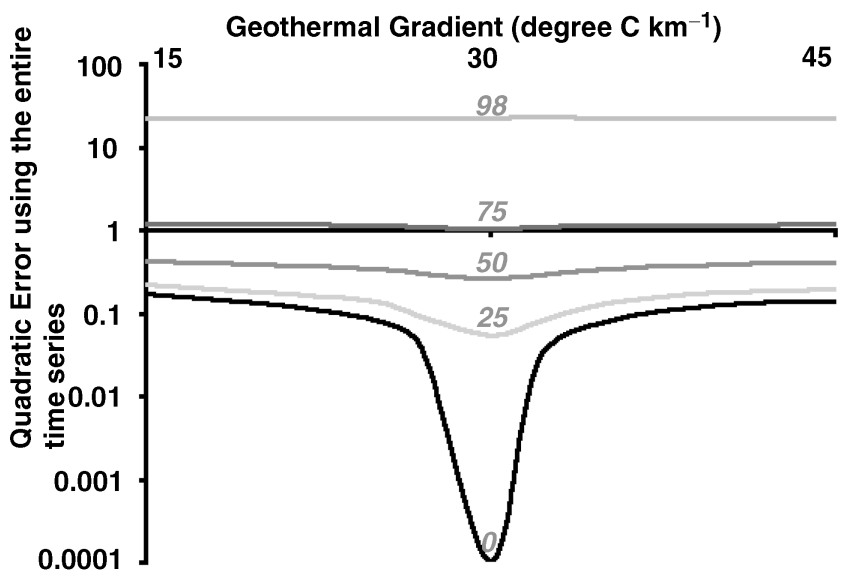

italic number next to a curve $=$ noise level in percentage

Figure 7. The variation of quadratic error based on the power spectrum of the seismograms to be part of the seismic inversion-basin modelling scheme (Fig. 1) at different noise levels. The error function is truncated to 0.0001 to avoid minus infinity in the noise-free graph.

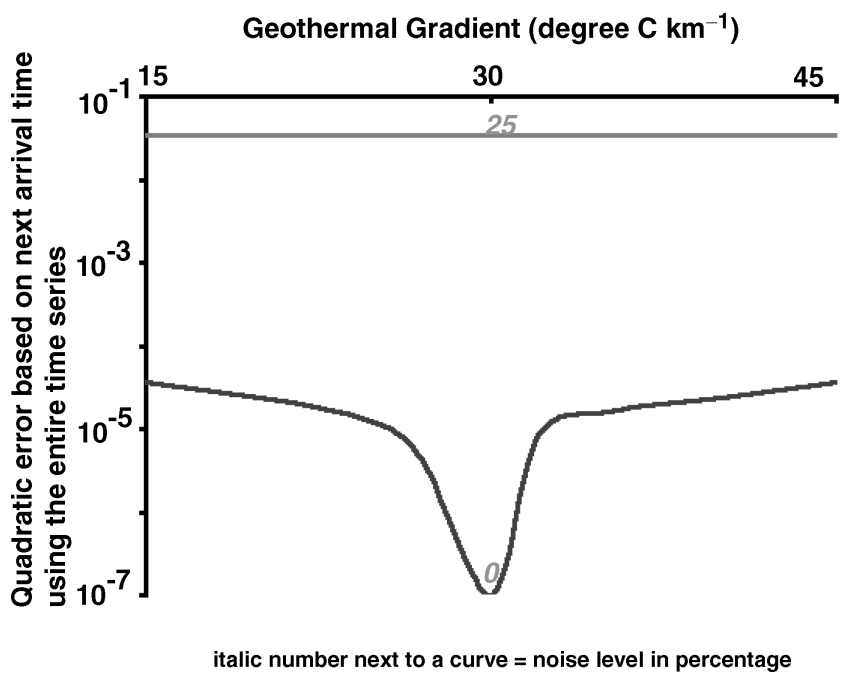

Figure 8. The variation of quadratic error based on the next arrival time using the entire time-series to be part of the seismic inversion-basin modelling scheme (Fig. 1). The error function is truncated to $10^{-7}$ to avoid minus infinity in the noise-free graph.

synthetic seismograms from our inversion is representative of the 'observed' one to even perform error analysis. An example of detailed data sets could be reservoirs from well developed oil and gas fields, e.g. those in Gulf of Mexico or North Sea. These will have enough wealth of data to explore the complex interaction of physics and chemistry of the sedimentary basins and perform inversion (Fig. 1). The physical and chemical processes are affected most strongly by the geothermal gradient in the deeper parts of the basin. Thus one might expect that a selected time interval from the deeper parts of the basin would be sufficient to determine a tectonic parameter, such as geothermal gradient. Fig. 9 shows that one can find a global minimum even in the presence of noise at 75 per cent level. If the linear correlation error uses the entire time-series, even if the Basin RTM simulation is coarser in certain intervals, then the global minimum value is lower. Experiments 3 and 4 are also performed using only partial time-series (from 1.0 to $1.742 \mathrm{~s}$ two-way traveltime).

\section{Basin RTM has detailed geological information in a limited region}

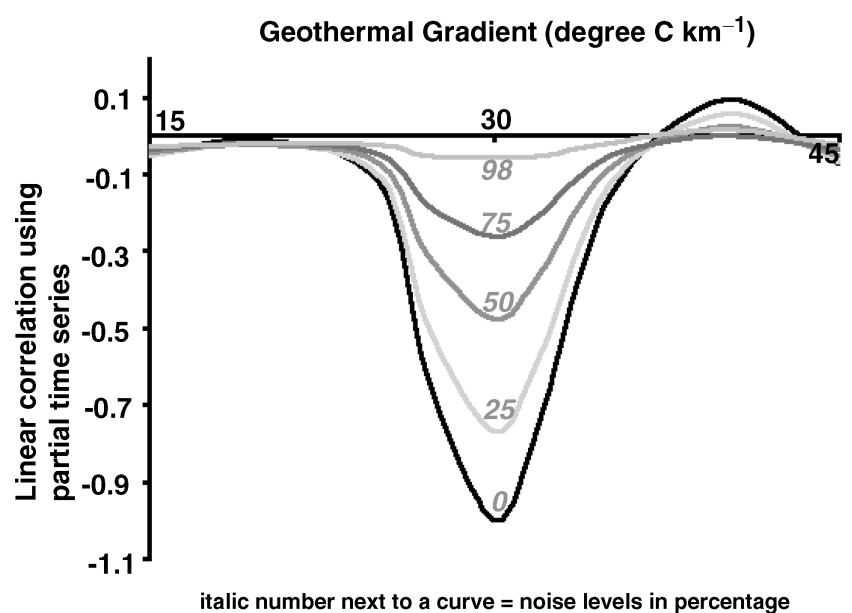

Figure 9. A linear correlation error when the first second of the 'observed' seismogram is not used in the error calculation. This is to highlight that error analysis is being done in a limited part, as there might not be enough detailed data for the shallower section of the sedimentary basin.

\section{Using only frequencies above $20 \mathrm{~Hz}$ - to remove the effect of low frequency noise from our basin modeling-inversion scheme}

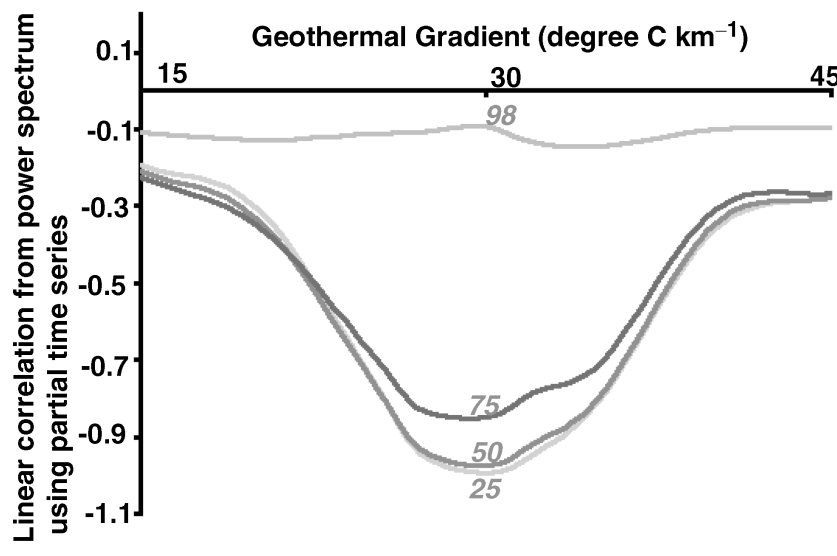

italic number next to a curve $=$ noise level in percentage

Figure 10. A linear correlation error based on the power spectrum using frequencies above $20 \mathrm{~Hz}$ and partial time-series. Error analysis in a limited frequency bandwidth can always be used to exclude noisy part of the data in the power spectrum.

As the noise level increases, the global minimum becomes shallower if linear correlation error is used (Fig. 9). In the case demonstrated here, the majority of noise added to the 'observed' data is less than $20 \mathrm{~Hz}$ (Fig. 4). If the linear correlation error is calculated using power spectra with only frequencies above $20 \mathrm{~Hz}$, then the algorithm yields a deeper global minimum even in the presence of appreciable noise (Fig. 10). Therefore, using power spectra for error analysis within a selective frequency bandwidth seems to enhance the results of our approach. Excluding frequencies from error analysis is beneficial only if the stacked noise has distinctly different frequency content than does the frequency bandwidth from the majority of the reflectors. Noise analysis is always carried out in seismic surveys that will enable us to determine power spectra of the noise and help 
in our technique when implemented on real data. When the power spectra of the noise and data are not clearly distinct (Fig. 4), then the linear correlation error using time-series (Fig. 5) along with power spectra (Fig. 6) should be used with care.

In real sedimentary basin examples, one can also use the existing the biostratigraphic information to calibrate the top horizon between the real seismogram data and modelled synthetic seismograms for the limited stratigraphic column that is being used in our inversion technique via time correction (Tandon et al. 1998) (Fig. 1). A time correction can also be used to correct for mismatch that might be caused by missing details from a shallower part of the basin as an input to the basin simulator.

\section{Experiment 3: observations and synthetic seismograms with different frequencies}

Attenuation and spherical divergence cause a loss of higher frequencies in the seismic reflection data. Attenuation and spherical divergence were not incorporated in the generation of synthetic seismograms used in this study. Even if these effects were included, it is likely that there always will be some difference in frequency content between the observed and synthetic data. To investigate these effects, the 'observed' and synthetic seismograms are constructed using 40 and $80 \mathrm{~Hz}$ Ricker wavelets, respectively. A $40 \mathrm{~Hz}$ difference between the 'observed' and synthetic seismograms approximates the loss of higher frequencies in a sedimentary basin due to attenuation and spherical divergence in the observed data. Fig. 11 shows that one can still find the global minimum at 75 per cent noise levels. Note that a $80 \mathrm{~Hz}$ Ricker wavelet is used for both 'observed' and synthetic seismograms in experiments 1,2 and 4 .

The range of frequencies for exploration seismology is generally 2-120 Hz (Sheriff \& Geldart 1995). Use of an $80 \mathrm{~Hz}$ Ricker wavelet constitutes a high-resolution, basin scale reflection seismic reflection survey. In shallow seismic reflection exploration (100-200 m), the dominant frequency can be as high as $1000 \mathrm{~Hz}$ (Buhnemann \& Holliger 1998), but we are more concerned in surveys that image even the deeper parts of the basin. A $40 \mathrm{~Hz}$ Ricker wavelet for the 'observed' data is chosen to mimic the effect of attenuation on the

\section{"Observed" and modeled seismograms have different frequencies}

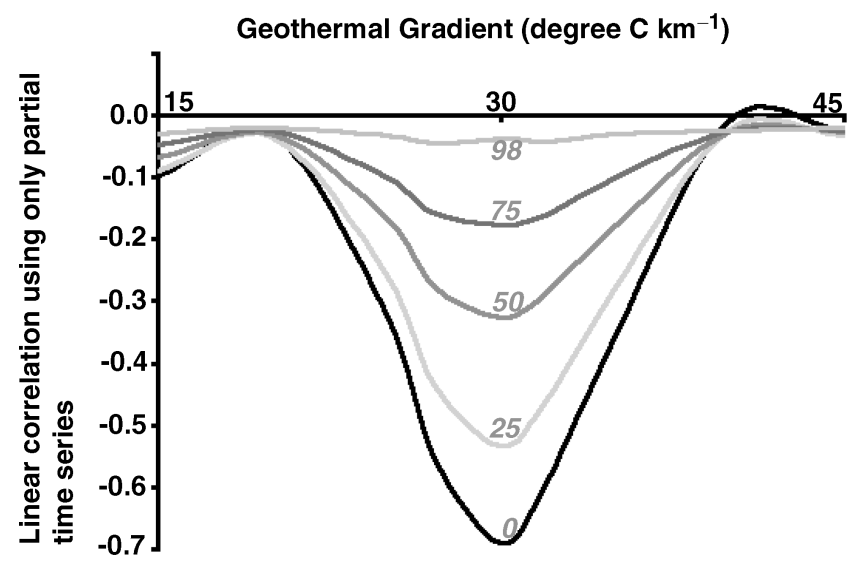

italic number next to a curve $=$ noise level in percentage

Figure 11. A linear correlation error based on the partial time-series. The 'observed' and synthetic seismograms are calculated using 40 and $80 \mathrm{~Hz}$ Ricker wavelets, respectively, to demonstrate the effect of attenuation. dominant frequency content. Typically, dominant frequencies observed in reflection seismic data lie between 5-50 Hz (Sheriff \& Geldart 1995).

\section{Experiment 4: basin data with missing lithologies}

In this section, we investigate the effects of resolution in the basin model and observed seismic reflection data. In general, resolution of the seismic reflection data is $1 / 4-1 / 8$ of the dominant wavelength of the source wavelet (Sheriff 1977). Deviations in the description of layering in the model on a similar or higher scale are expected to affect the algorithm. The purpose of this experiment is to determine whether the algorithm fails when the model response does not correspond exactly to the layering of the stratigraphic column sampled by the mechanical waves as in the 'observed' seismic data. It is unlikely that the sediment density and $P$-wave profiles from the basin simulator exactly correspond to the layering of the actual rock. Most likely, the basin model will not be able to simulate all the heterogeneities sampled by the seismic waves in a controlled source experiment.

To generate an incomplete basin model input, we removed every eighth layer from the entire model input data and the thickness of the seventh layer was increased to compensate for the removed layer. Such missing layers not only result in the absence of reflectors in the synthetic seismogram, but also lead to a perturbed velocity/depth curve. It was found that a global minimum can be determined using linear correlation error based on power spectra (with only frequencies above $20 \mathrm{~Hz}$ ) in the presence of 75 per cent noise level (Fig. 12).

In a similar experiment, every fourth layer from the basin input was removed from the basin input data. Fig. 13 shows that the correct global minimum can approximately be determined using the linear correlation error of power spectra (frequencies above $20 \mathrm{~Hz}$ ). Therefore, the layering, the bulk density, and $P$-wave velocity profiles generated by Basin RTM need not be perfectly matched even in a limited stratigraphic column for our algorithm to work.

\section{Modeled seismogram has 7/8th number of layers $(\mathbf{8 7 . 5 \% )}$ compared to "observed" data}

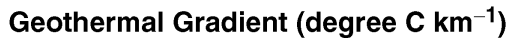

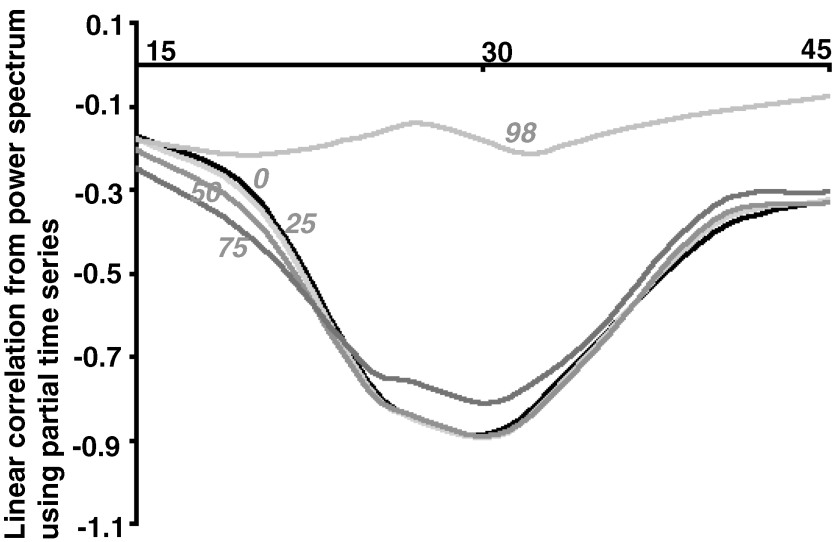

italic number next to a curve $=$ noise level in percentage only frequencies above $20 \mathrm{~Hz}$ are used for error analysis

Figure 12. A linear correlation error based on the power spectrum using the partial time-series and frequencies above $20 \mathrm{~Hz}$. Every eighth lithologic layer was missed in the basin simulator input to test the robustness of the procedure to insufficient lithologic delineation. 
Modeled seismogram has 3/4th number of layers $(75 \%)$ compared to "observed" data

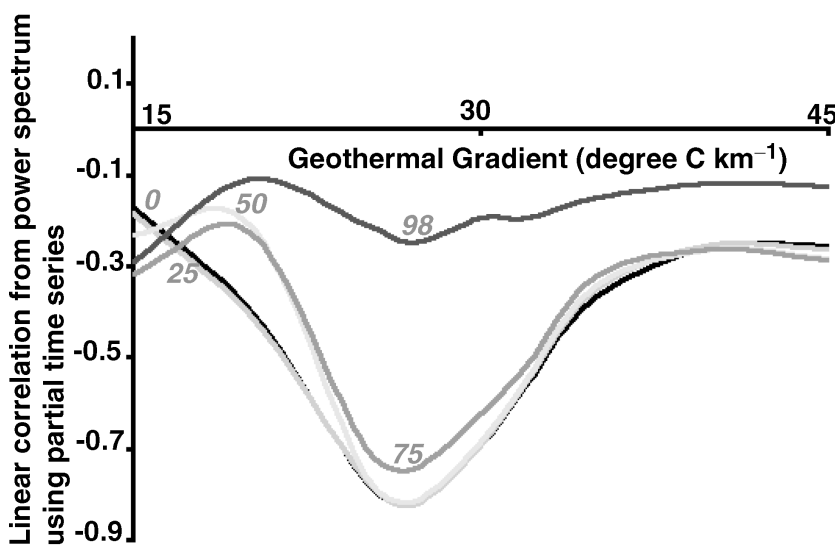

italic number next to a curve $=$ noise level in percentage only frequencies above $20 \mathrm{~Hz}$ are used for error analysis

Figure 13. The same as Fig. 12 except that every fourth layer was missed in the basin simulator input.

\section{CONCLUSIONS}

The basin simulation-enhanced seismic inversion method has been shown to be a viable approach to delineating the state of the subsurface and the heterogeneities formed in the sedimentary basin. The method has the potential for greatly reducing the cost and accuracy of seismic exploration and understanding of the rheological evolution of sedimentary basins in response to varying tectonic parameters. However, the present study has even wider implications. We suggest that it provides an approach for integrating many types of basin data (well logs, geochemical, core analysis and thermal data) in addition to seismic data to yield one integrated approach by generalizing the algorithm of Fig. 1. Thereby, basin modelling enhances the quality of data analysis and enhances the results of basin simulation all in one unified, automatable and parallelizable computational approach for data assimilation.

The present study is only preliminary and presents a new methodology being developed. This study lays down the theoretical framework for future work where basin modelling and multiple data sets are fully integrated. Further studies will include actual examples from sedimentary basins in different parts of the world. The basin simulation was carried out in one, and not three, spatial dimensions - limiting the level of reliability. The basin model used, Basin RTM, while arguably the most comprehensive model available at this writing, still could benefit from the addition of other processes and the refinement of its rheological, hydrologic, and reaction rate laws. Furthermore, limitations due to the intensive computational demands of our procedure will place some restrictions on its widespread use for the next several years. However, this is a first step in understanding a detailed, complex evolution of heterogeneities in sedimentary basins that are capable of being imaged by reflection seismic data and can be used for geoinfomatics of sedimentary basins.

Another concern that should be addressed in future research pertaining to our method or similar methods is the uniqueness of the inversion/prediction. Can there be other geothermal histories that give the same seismic data? Also, do the predictions reflect the true content of the data or is the latter masked by an incomplete model or one based on an erroneous rheological or other rate laws? One must also remember that even the most comprehensive model is never a complete description of the complexity that is present in a sedimentary basin. Finally, methods that simultaneously provide an estimate of uncertainty in the inversion and risk in the strategy based on the predictions should be used (Tuncay \& Ortoleva 2003).

Our algorithm works even when the model has a detailed stratigraphic description in a limited region with incomplete stratigraphic data (12.5-25 per cent absent). As a result of the shape of the geothermal gradient-linear correlation error measure, the iterative quadratic fitting is a more efficient optimization technique than simulated annealing. Of all the error measures used, linear correlation error of the power spectrum between the observed and synthetic seismograms is the most ideal choice. If the power spectrum of noise and dominant reflectivity in the observed data are different, then the error analysis in a selected frequency bandwidth makes our algorithm even more robust.

Our methodology is viable even (1) in the presence of large amounts of low-frequency noise in the seismic data, (2) when information concerning detailed stratigraphic layering is confined to a limited region and (3) frequency content of the observed and predicted seismograms differ. The necessary requirement for the methodology to succeed is the availability of detailed geological information in a sedimentary basin. In earth sciences, the maximum wealth of geological, geochemical and geophysical data for sedimentary basins has been collected by academic, oil and gas, mining industry endeavours for centuries. Therefore, approaches that attempt to put all these data sets together in a rigorous fashion and their potential for prediction should be fully explored.

\section{ACKNOWLEDGMENTS}

This work was supported by the National Energy Technology Laboratory (NETL) of the US Department of Energy through contract no. DE-AC26-00NT40689. We thank Dr Frances Toro (NETL) for her constructive critiques and encouragements. We thank Dr Juan Lorenzo (Louisiana State University) for providing us with his synthetic seismogram programme and Dr Everett Carter (Taygeta Scientific Inc., www.taygeta.com) for the use of the simulated annealing code. Power spectra was calculated using FFTW, a $C$ subroutine library for computing discrete Fourier transforms (www.fftw.org). Support from Publications and Outreach office at COAS to KT is highly appreciated. Comments from an anonymous reviewer and the Associate Editor, Steven Ward are greatly appreciated as they improved the manuscript.

\section{REFERENCES}

Basu, A. \& Frazer, L.N., 1990. Rapid determination of critical temperature in simulated annealing inversion, Science, 249, 1409-1412.

Berryman, J.G., 1986. Effective medium approximation for elastic constants of porous solids with microscopic heterogeneity, J. appl. Phys., 59, 11361140 .

Blatt, H. \& Tracey, R., 1995. Petrology, Igneous, Sedimentary and Metamorphic, W.H. Freeman \& Co.

Boadu, F.K., 1998. Inversion of fracture density from seismic velocities using artificial neural networks, Geophysics, 63, 534-545.

Bour, O. \& Lerche, I., 1994. Numerical modelling of abnormal fluid pressures in the Navarin Basin, Bering Sea, Marine and Petroleum Geology, 11, 491-500.

Buhnemann, J. \& Holliger, K., 1998. Comparison of high-frequency seismic sources at the Grimsel test site, central Alps, Switzerland, Geophysics, 63, 1363-1370.

Cabrera, G.R., 1996. Application of geostatistical techniques to 3-D, 3-C seismic and core studies to estimate reservoir porosity in the lower Nisku 
interval at Joffre Field, Alberta, Canada, Master's thesis, Colorado School of Mines, Colorado.

Douze, E.J., 1964. Signal and noise in deep wells, Geophysics, 29, 721-732.

Douze, E.J. \& Laster, S.J., 1979. Seismic array noise studies at Roosevelt Hot Springs, Utah geothermal area, Geophysics, 44, 1570-1583.

Forbes, P.L., Ungerer P. \& Mudford, B.S., 1992. A two dimensional model of overpressure development and gas accumulation in Venture Field, Eastern Canada, AAPG Bulletin, 76, 318-338.

Gordon, D.S. \& Flemings, P.B., 1998. Generation of overpressure and compaction-driven fluid flow in a Plio-Pleistocene growth-faulted basin, Eugene Island 330, offshore Louisiana, Basin Research, 10, 177-196.

Ingber, A.L., 1993. Simulated annealing: Practice versus theory, J. Mathl. Comput. Modeling, 18, 29-57.

Jolly, R.N. \& Mifsud, J.F., 1971. Experimental studies of source-generated seismic noise, Geophysics, 36, 1138-1149.

Kirkpatrick, S., Gelatt, C.D., Jr \& Vecchi, M.P., 1983. Optimization by simulated annealing, Science, 220, 671-680.

Klimentos, T. \& McCann, C., 1983. Relationships among compressional wave attenuation, porosity, clay content, and permeability in sandstones, Geophysics, 55, 998-1014.

Larner, K., Chambers, R., Yang, M., Lynn, W. \& Wai, W., 1983. Coherent noise in marine seismic data, Geophysics, 48, 854-886.

Lerche, I., 1991. Inversion of dynamical indicators in quantitative basin analysis models. I. Theoretical considerations, Mathematical Geology, 23, 817-832.

Lorenzo, J.M. \& Hesselbo, S., 1996. Seismic-to-well correlation at ODP 150 continental slope sites, Proc. Ocean Drilling Program, Scientific Results, 150, 293-307

Luo, X. \& Vasseur, G., 1995. Modelling of pore pressure evolution associated with sedimentation and uplift in sedimentary basins, Basin Research, 7, $35-52$.

Luo, X. \& Vasseur, G., 1996. Geopressuring mechanism of organic matter cracking: numerical modeling, AAPG Bulletin, 80, 856-873.

Luo, X., Vasseur, G., Pouya, A., Lamoureux-Var, V. \& Poliakov, A., 1998. Elastoplastic deformation of porous medium applied to the modeling of compaction at basin scale, Marine and Petroleum Geology, 15, 145162 .

McPherson, B.J.O.L. \& Bredehoeft, J.D., 2001. Overpressures in the Uinta Basin, Utah: Analysis using a three-dimensional basin evolution model, Water Resources Research, 37, 857-871.

McPherson, B.J.O.L. \& Garven, G., 1999. Hydrodynamics and overpressure mechanisms in the Sacramento Basin, California, Am. J. Sci., 299, 429466.

McQuillin, R., Bacon, M., \& Barclay, W., 1984. An introduction to seismic interpretation: reflection seismics in petroleum exploration, Graham and Trotman, London.

Mallick, S., Craft, K.L., Meister, L.J. \& Chambers, R.E., 1998. Determination of the principal directions of azimuthal anisotropy from P-wave seismic data, Geophysics, 63, 692-706.

Maubeuge, F. \& Lerche, I., 1993. A north Indonesian basin: Geothermal and hydrocarbon generation histories, Marine and Petroleum Geology, 10, 231-245.

Maubeuge, F. \& Lerche, I., 1994. Geopressure evolution and hydrocarbon generation in a north Indonesian basin: two-dimensional quantitative modeling, Marine and Petroleum Geology, 104, 104-115.

Murphy, W.F., 1982. Effects of partial water saturation on attenuation in Massilon sandstone and Vycor porous glass, J. acoust. Soc. Am., 71, 14581468.

Nagano, K., 1998. Crack-wave dispersion at a fluid-filled fracture with lowvelocity layers, Geophys. J. Int., 134, 903-910.

Otten, R.H.J.M. \& van Ginneken, L.P.P.P., 1989. The Annealing Algorithm, Kluwer Academic Publishers.

Payne, D.F., Tuncay, K., Park, A., Comer, J. \& Ortoleva, P., 2000. A reactiontransport-mechanical approach to modelling the interrelationships between gas generation, overpressuring, and fracturing-Implications for the Upper Cretaceous natural gas reservoirs of the Piceance Basin, Colorado, AAPG Bulletin, 84, 545-565.

Person, M. \& Garven, G., 1992. Hydrologic constraints on petroleum gen- eration within continental rift basins: Theory and application to the Rhine Graben, AAPG Bulletin, 76, 468-488.

Person, M., Toupin, D. \& Eadington, P., 1995. One-dimensional models of groundwater flow, sediment thermal history and petroleum generation within continental rift basins, Basin Research, 7, 81-96.

Peterson, R.A., Fillipone, W.R. \& Coker, E.B., 1955. The synthesis of seismograms from well log data, Geophysics, 20, 516-538.

Press, W.H., Teukolsky, S.A., \& Vetterling, W.T., 1993. Numerical recipes in C: The art of scientific computing, Cambridge University Press, Cambridge.

Ramos, A.C.B. \& Davis, T.L., 1997. 3-D AVO analysis and modeling applied to fracture detection in coalbed methane reservoirs, Geophysics, 62, 16831695.

Roberts, S.J. \& Nunn, J.A., 1995. Episodic fluid expulsion from geopressured sediments, Marine and Petroleum Geology, 12, 195-204.

Rothman, D.H., 1985. Nonlinear inversion, statistical mechanics, and residual statics estimation, Geophysics, 50, 2784-2796.

Rothman, D.H., 1986. Automatic estimation of large residual statics correction, Geophysics, 51, 337-346.

Schlumberger Log Interpretation, 1972. Principles/Applications, Schlumberger Educational Services.

Schneider, F., Potdevin, J.L., Wolf, S. \& Faille, I., 1996. Mechanical and chemical compaction model for sedimentary basin simulators, Tectonophysics, 263, 307-317.

Sen, M.K. \& Stoffa, P.L., 1991. Nonlinear one-dimensional seismic waveform inversion using simulated annealing, Geophysics, 56, 1624-1638.

Sen, M.K. \& Stoffa, P.L., 1995. Global optimization methods in geophysical inversion, in Advances in Exploration Geophysics 4, ed. Berkhout, A.J., Elsevier.

Sheriff, R.E., 1977. Limitations on resolution of seismic reflections and geologic detail derivable from them. in Seismic Stratigraphy-Applications to Hydrocarbon Exploration, Vol. 26, pp. 3-14, ed. Payton, C.E., Am. Assoc. Pet. Geol. Mem.

Sheriff, R.E. \& Geldart, L.P., 1995. Exploration Seismology, Cambridge University Press.

Suetnova, E. \& Vasseur, G., 2000. 1-D modelling rock compaction in sedimentary basins using a visco-elastic rheology, Earth planet. Sci. Lett., 178, 373-383.

Szu, H. \& Hartley, R., 1987. Fast simulated annealing, Physics Letters A, 122, 157-162.

Tandon, K., Lorenzo, J.M. \& de la Linde Rubio, J., 1998. Timing of rifting in the Alboran Sea Basin - Correlation of borehole (ODP Leg 161 and Andalucia A-1) to seismic reflection data: implications for basin formation, Marine Geology, 144, 275-294

Tuncay, K. \& Ortoleva, P., 2001. Salt tectonics as a self-organizing process: a three dimensional reaction, transport and mechanics model, J. geophys. Res., 106, 803-818.

Tuncay, K. \& Ortoleva, P., 2003. Quantitative basin modeling: Present state and future developments towards predictibility, Geofluids, in press.

Tuncay, K., Park, A. \& Ortoleva, P., 2000a. Sedimentary basin deformation: An incremental stress approach, Tectonophysics, 23, 77-104.

Tuncay, K., Park, A. \& Ortoleva, P., 2000b. A forward fracture model to predict fracture orientation and properties, J. geophys. Res., 105, 16719 16735.

Turcotte, D.L. \& Schubert, G., 1982. Geodynamics: Applications of Continuum Physics to Geological Problems, John Wiley \& Sons.

Ungerer, P., Burrus, J., Doligez, B., Chenet, P.Y. \& Bessis, F., 1990. Basin evaluation by integrated two-dimensional modeling of heat transfer, fluid flow, hydrocarbon generation, and migration, AAPG Bulletin, 74, 309335

Ursin, B., de Matos Neto, J.B. \& Posani, M.J., 1996. Seismic reflection estimation with colored noise, Journal of Seismic Exploration, 5, 5162.

Yilmaz, O., 1987. Seismic Data Processing, Investigations in Geophysics, Vol. 2, ed. Doherty, S.M., Society of Exploration Geophysicists.

Yu, Z., Lerche, I. \& Bour, Q., 1995. Inversion of dynamical indicators in quantitative basin analysis models. III. Multiwell information and twodimensional case histories, Mathematical Geology, 27, 41-68. 
Wang, C. \& Xie, X., 1998. Hydrofracturing and episodic fluid flow in shalerich basins-A numerical study, AAPG Bulletin, 82, 1857-1869.

Wieck, J., Person, M. \& Strayer, L., 1995. A finite element method for simulating fault block motion and hydrothermal fluid flow within rifting basins, Water Resources Research, 31, 3241-3258.

Zhao, K. \& Lerche, I., 1993. Inversion of dynamical indicators in quantitative basin analysis models. II. Synthetic tests and a case history using dynamical indicator tomography, Mathematical Geology, 25, 107-123.

\section{APPENDIX}

Let $s(t)$ be the synthetic seismogram generated by the basin simulator at a geothermal gradient of $30{ }^{\circ} \mathrm{C} \mathrm{km}^{-1}, n(t)$ is the random noise generated and $\operatorname{swn}(t)$ is the seismogram with random noise added to $s(t)[\operatorname{swn}(t)=s(t)+n(t)]$. The random noise $n(t)$ is generated as follows:

$n(t)=B \sum_{n=N_{0}}^{N_{\max }} \frac{\xi_{n}}{1+m} \sin \left(\frac{2 \pi n t}{T}+\phi_{n}\right)$,

where $m$ determines the harmonic order ranging from $N_{0}$ to $N_{\max }$, $T$ is the total time period for the time-series, $B$ scales the amplitude of the random noise generator according to the signal $s(t)$ and is the randomly generated phase for each Fourier component using random numbers. The parameter $B$ is used to determine the noise level $(N L) . N L$ is related to $B$ via

$N L^{2}=\frac{\int n^{2}(t) d t}{\int s w n^{2}(t) d t}$. 


\section{Appendix B}

Tuncay, K., A. Park, and P. Ortoleva. 2000. Sedimentary basin deformation: An incremental stress rheology approach. Tectonophysics, Vol. 323, 77-104. 


\title{
Sedimentary basin deformation: an incremental stress approach
}

\author{
K. Tuncay, A. Park, P. Ortoleva * \\ Laboratory for Computational Geodynamics, Department of Chemistry, Indiana University, Bloomington, IN 47405, USA
}

Received 25 January 2000; accepted for publication 14 March 2000

\begin{abstract}
A key component of sedimentary basin evolution is the spatial distribution and temporal variation of stress and deformation. The many deformation processes (poroelasticity, fracturing, irreversible nonlinear viscosity, and pressure solution) are inextricably bound in a tightly coupled network which, in turn, is coupled to a myriad of basin diagenetic, thermal and hydrologic processes. In the approach presented here, the various deformation processes are integrated through an incremental stress approach. Together with mass, momentum and energy conservation, this approach yields a complete, fully coupled basin model that captures basin and fault phenomena that are beyond the scope of simpler or decoupled models.

Many of the most interesting basin phenomena are not only dependent on multiple, coupled processes but also are fundamentally three-dimensional. To address this three-dimensional complexity, we have developed a numerical simulator using a moving, adapting, accreting finite element grid which is allowed to deform and to grow and adapt with the addition of sediment to capture smaller sedimentary features.

As a result, our fully coupled, comprehensive model allows one to solve a number of key problems in basin and fault dynamics. These include compaction, fractured reservoir and compartment genesis and dynamics. Examples illustrating these applications are presented for idealized systems and the Piceance Basin (Colorado) and the Permian Basin (West Texas). The incremental stress rheology is found to be a powerful formalism for integrating basin hydrology, diagenesis and mechanics. (c) 2000 Elsevier Science B.V. All rights reserved.
\end{abstract}

Keywords: deformation; rheology; sedimentary basin; stress

\section{Introduction}

Reconstructing the stress and deformation history of a sedimentary basin is a challenging and important problem in the geosciences and a variety of applications. The latter include petroleum exploration, reserve assessment and production, and earthquake hazard reduction. Progress in this field has been hampered by the absence of an integrated mechanical modeling approach set within the wider context of the coupled reaction, transport and mechanical (RTM) dynamics of a basin. This

\footnotetext{
* Corresponding author. Fax: +1-812-855-8300.
}

integration and implementation as a three-dimensional simulator are the major goals of this work.

The strongly coupled nature of the deformation problem may be understood in terms of the feedbacks underlying crustal dynamics. For example, pore fluid pressure affects stress, stress changes can lead to fracturing, and fracturing can affect pore fluid pressure. Similarly, stress can affect mineral solubility, causing mineral dissolution which, in turn, can affect rock rheology and, therefore, stress. Clearly, basin deformation analysis requires accounting of the coupling among the many operating, interacting RTM processes. 
It has become clear over the past two decades that many geological phenomena can only be explained via strongly coupled RTM models (see reviews in Haase et al. 1980; Ortoleva, 1979, 1990, 1994a; Nicolis and Nicolis, 1987; Ortoleva, et al., 1987a,b; Turcote, 1992). Therefore, modeling sedimentary basin dynamics requires a fully coupled, integrated approach. In the approach presented here, integration is achieved through an incremental stress (Zienkiewicz and Cormeau, 1974; Rice, 1975) approach.

The goal of the modeling presented here is to calculate the evolution of the distribution within a basin of the set of descriptive variables characterizing its internal state:

- rock texture and mineralogy

- fluid properties

- temperature

- rock deformation
- fracture network characteristics

- stress.

These properties respond over geological time to their interactions among each other and via the influence of the basin's surroundings. These features are summarized in Fig. 1. The interaction with the surroundings provides the boundary conditions to which the equations of mass, energy and momentum conservation must be subjected to arrive at the evolution of the basin. In this way, basin analysis becomes the delineation of the RTM basin dynamical system and its response to the constraints imposed at the boundaries. As the laws for the basin RTM processes are nonlinear in the descriptive variables, one expects this response to be extremely rich (Ortoleva, 1993, 1994a,c, 1998; Dewers and Ortoleva, 1994).

Other models do exist and have been used to gain valuable insights into the basin system.

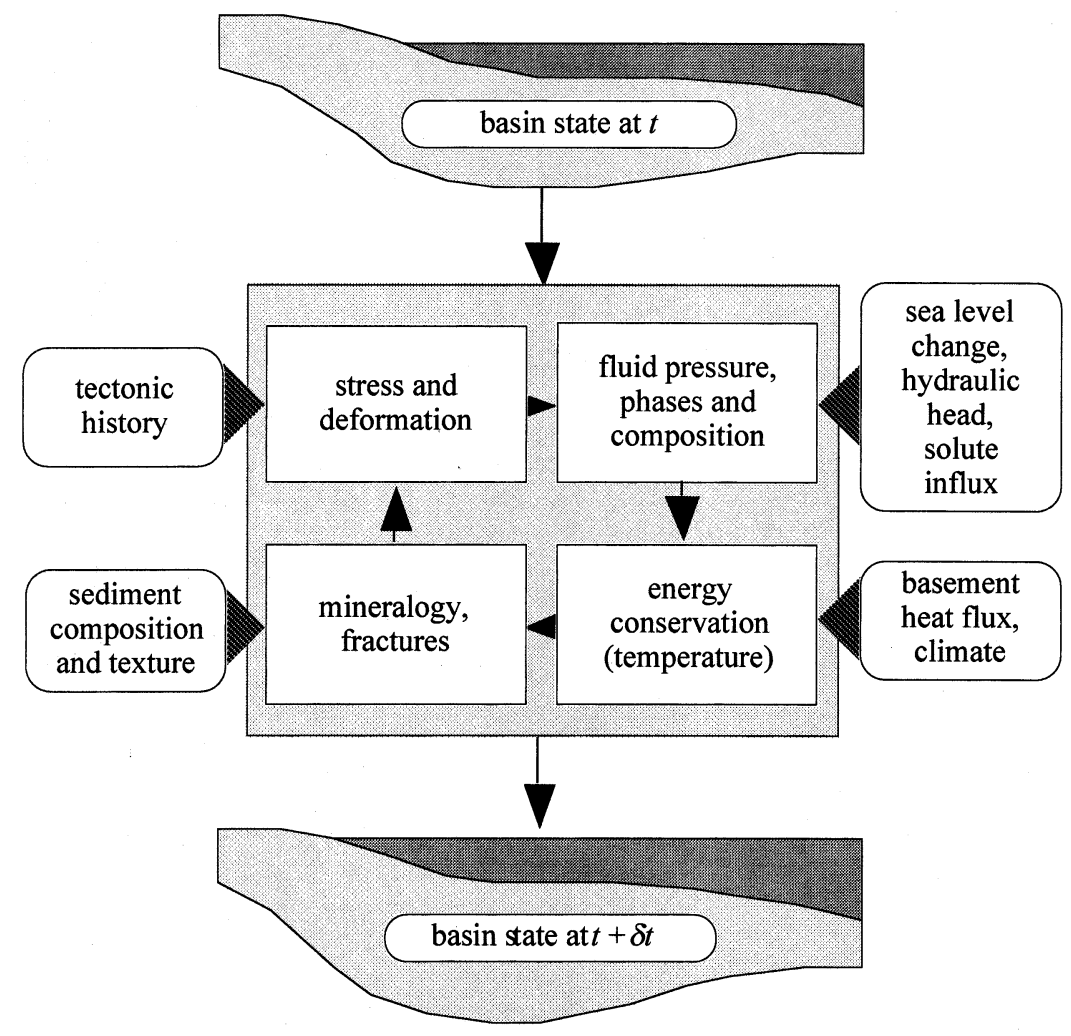

Fig. 1. Flow chart showing how the interplay of geologic data and physico-chemical (i.e., reaction-transport-mechanical) process modules evolve the basin over a computational time interval $\delta t$. 
However, the many strongly coupled basin RTM processes as suggested in Fig. 1 require a comprehensiveness and three-dimensional approach that goes far beyond these two- or three-process, typically two-dimensional models (Ungerer et al., 1990; Larson et al., 1993; Maubeuge and Lerche, 1993, 1994; Dewers and Ortoleva, 1994; Sonnenthal and Ortoleva, 1994; Roberts and Nunn, 1995; Luo and Vasseur, 1996; Schneider et al., 1996; Luo et al., 1998; Wang and Xie, 1998). To be effective, models must be fully three-dimensional to capture basin sedimentological geometry, fracture orientation, changing compression/extensional tectonic regimes and basement heat flux anomalies.

Fracture mediated petroleum migrations from reservoirs are key aspects of the dynamic petroleum (or other crustal fluid) system. In most of the existing basin evolution models, it is assumed that rocks fracture when the fluid pressure exceeds a certain fraction of lithostatic stress (Ghaith et al., 1990; Maubeuge and Lerche, 1993, 1994; Chen et al., 1994; Sonnenthal and Ortoleva, 1994; Roberts and Nunn, 1995; Wang and Xie, 1998). This assumption essentially eliminates the dependence of fracturing on lithologic properties. In this study, we find that fracturing strongly depends on the rock texture, i.e., mineral composition, grain size, porosity, etc. For example, the difference in neighboring sandstone and shales with respect to their fracture response is commonly observed (Kulander et al., 1979; Segall and Pollard, 1983; Hancock et al., 1984; Lorenz et al., 1991; Gross, 1993; Fischer et al., 1995; Wu and Pollard, 1995; Payne et al., 2000). Although fracturing can occur in almost any type of rock, they are more common in brittle rocks (Mallory, 1977). Furthermore, fractures in a brittle lithology commonly discontinue at the interface of more ductile lithologies (Engelder and Geiser, 1980). Another observation is that fracture spacing is strongly dependent on bed thickness and lithology (Harris et al., 1960; Nickelsen and Hough, 1967; Gross, 1993; Fischer et al., 1995; Wu and Pollard, 1995).

Another limiting assumption made in other studies is that there exists a simple dependence of porosity on effective stress (Ungerer et al., 1990; Maubeuge and Lerche, 1993, 1994; Roberts and Nunn, 1995; Luo and Vasseur, 1996; Schneider et al., 1996; Wang and Xie, 1998). This results in a very smooth porosity profile and, once again, eliminates the importance of the lithologic dependence of rock properties. In our approach, porosity is obtained by solving the mass conservation equation for the solids using the rock deformation velocity computed by a multi-process, incremental stress rheology. Since, in our approach, the elastic, viscous and yield parameters are functions of texture, the evolution of porosity and stress are strongly coupled and thereby computed self-consistently. As a result, shales tend to have lower porosity and higher least-compressive stress than sandstones. The small grain size combined with low porosity results in very low permeability and, thus, these layers can form efficient seals. Furthermore, our results show that low shear viscosity/bulk viscosity ratio makes fracturing very unlikely in the absence of flexure or extreme overpressuring. Mechanisms of overpressuring include petroleum generation, fluid thermal expansion and compaction. In the numerical results presented here, we illustrate the episodic fracturing of a seal layer resulting from a high rate oil generation.

In other basin evolution simulators, fracture permeability is assumed to be isotropic. This can be attributed to the fact that, for an accurate description of fracture orientations, the full stress tensor must be known. In our approach, a representative set of putative fractures of a range of orientations is introduced. The time dependent properties of each realized fracture are calculated by using the stress component normal to its fracture plane, pressure and rock properties. The anisotropic fracture permeability is obtained using the predicted fracture network statistics (see Tuncay et al., 2000 for details).

Another effect that is always disregarded is the volumetric strain caused by fracturing. As fractures open, the overall rock volume increases and fluid pressure decreases (due to flow and increase in pore volume). This reduces the rate of fracture growth. Therefore, fracturing is a self-limiting process. In summary, a fully coupled deformation/ hydrologic/fracturing model is required to capture the co-evolution of these fracture enhancing and limiting factors.

Although one- and two-dimensional studies give 
hints into the dynamics of basin evolution, a threedimensional basin simulator is necessary to take into account all geometric effects. This becomes extremely important when fracturing is due mainly to flexure and the direction of tectonic compressive/extension is changing over the basin's history. Fracture networks provide a pathway for fluid flow and, especially in a layered medium, fluids can move laterally. This can only be taken into account by a three-dimensional basin simulator with a stress/deformation solver that can capture the nonplanar layers (notably domes) and the strong variations in rheology from layer to layer (e.g., sandstones versus shales or salt). The fundamentally three-dimensional nature of these systems is further enhanced as preferred fracture orientation induces anisotropic permeability tensor that can strongly influence the direction of fluid flow. To our knowledge, existing basin evolution simulators are mostly one- or two-dimensional (Ungerer et al., 1990; Larson et al., 1993; Maubeuge and Lerche, 1993; 1994; Roberts and Nunn, 1995; Luo and Vasseur, 1996; Schneider et al., 1996; Luo et al., 1998; Wang and Xie, 1998).

Although the history of basement heat flux, sedimentation and erosion rates, and subsidence and upheaval rates are among the most important parameters that effect the basin evolution, some basin simulators start with a predefined grid (Schneider et al., 1996). In other words, two basins with the same final thickness and sediments but different histories are assumed to behave similarly. It is well known that overpressuring, a key factor in fracturing and other deformation processes, correlates with sedimentation rate (Dewers and Ortoleva, 1994; Wang and Xie, 1998; Ortoleva, 1998).

In the classical flexure analysis, the history of deformation and its relation to the lithologic properties is ignored although present-day flexure is often a poor indication of fracturing. If the rate of flexure development was very slow, then rocks could have deformed continuously, depending on viscosities, and there would be no fracturing. Flexure can occur without fracturing early in a sediment's evolution, i.e., when it is poorly lithified or if it has inherently ductile behavior (as for organic-rich shales, rock salt or anhydrates).
Therefore, the next generation of basin evolution simulators should be not only three-dimensional, but should also consider the history of sedimentation, erosion, tectonic and thermal evolution and their interplay with deformation.

\section{Modeling concept}

The conservation laws are universal; it is the differences in the history of the evolving boundary conditions that give a basin its individual character. Thus, one might state that the geology is in the boundary conditions and the physics and chemistry imply the form of the conservation equations.

In this study, we attempt to demonstrate that the integration of mechanics into a basin RTM model can be done most effectively using an incremental stress approach (Ortoleva, 1994a, 1998). In analogy with the classic theory of chemical kinetics, the total rate of strain $\epsilon$ is written as a sum of terms, each accounting for a particular process $\left(j=1,2, \ldots N_{d}\right)$ for a system with $N_{d}$ deformation processes:

$\underline{\underline{\dot{\epsilon}}}=\sum_{j=1}^{N_{d}} \underline{\underline{\dot{\epsilon}}}^{(j)}$.

Such processes included in this study are:

- poroelasticity

- continuous, irreversible rock deformation

- fracturing

- pressure solution

Recently, we have studied contributions of gouge, and coal devolitilization shrinkage/cleating to the total rate of strain (Ortoleva, 1998; Ozkan et al., 1998; Ozkan and Ortoleva, 1999b). Each of the deformation processes includes a variety of possibilities. For example, fracturing may be induced by flexure or elevated fluid pressure. Devolitilization in coals, dehydration, and thermally-induced shrinkage may also lead to fractures.

The outstanding contribution of incremental stress theory is that the total rate of strain is expressible as a linear combination of rate of strains from different process due to the fact that it represents a relation among infinitesimal changes. Multiplying both sides of Eq. (1) by a 
small time increment $d t$, the equation states that an infinitesimal deformation is expressed as a sum of infinitesimal deformation due to various processes. This type of statement has been the basis of chemical kinetics over the past century and has entered in fundamental physics at least as far back as Newton who recognized that the rate of change of momentum was equal to a linear combination of forces.

The individual rate of strain terms on the righthand side of Eq. (1) depend on the full suite of rock textural and fluid properties as well as the macroscopic stress. It is through this dependence and the coevolution of rock deformation and of these variables that the full coupling of all processes is accounted for in our model. As the rates $\underline{\underline{\dot{\epsilon}}}^{(j)}$ typically vary stronger than linearly with these variables, the basin is a nonlinear dynamical system.

We suggest that rigorous models of rock behavior should be of the Morkov type - i.e., the rate of change of rock state should only depend on the instantaneous rock state and not on prior history. Stress and strain are related through rock rheology, to rock texture $\Theta$ (grain size, shape, packing, mineralogy, and fracture length, aperture and orientation statistics). Pressure solution and grain breakage imply that the rate of change of $\Theta$ depends on stress, denoted $\sigma$. If $\Theta$ satisfies the different equation $d \Theta / d t=G(\Theta, \sigma)$ then, in principle, $\Theta(t)$ is a functional of $\sigma$, i.e., depends on $\sigma\left(t^{\prime}<t\right.$, i.e., the stress history: $\Theta=\Theta[\sigma]$. As rheology depends on $\Theta$ we see that $\Theta[\sigma]$ reflects the entire prior stress history and not just the instantaneous value of $\sigma$. Clearly, however, this "memory" in a theory wherein $\Theta$ is not coevolved with $\sigma$ is an artefact of the incompleteness of a rock deformation model that attempts to avoid coevolving $\Theta$ with stress. While there are many stress-strain histories that could lead to the instantaneous state of a rock, only the latter is key to predicting its failure and other behavior.

Our model uniquely accounts for the changing rock rheological parameters that accompany the changing texture (e.g., grain size, mineralogy and porosity) and fracture network properties (length, aperture, number density and orientation statistics). The bulk, shear and effective stress coeffi-

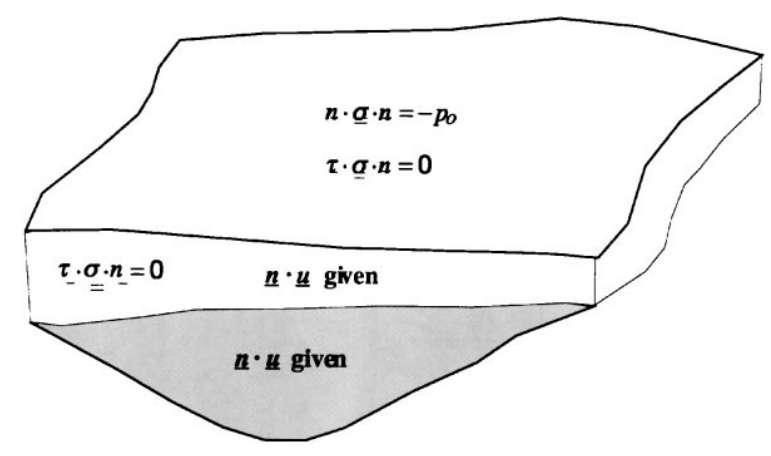

Fig. 2. Conditions at the boundary of basin simulation domain allow for imposition of ocean bottom normal pressure $p_{o}$ and

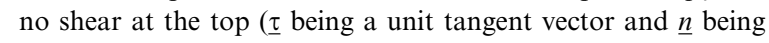
an outward pointing unit normal vector with respect to the basin boundary). At the bottom, the tectonic history fixes the evolution of the deformation velocity $\underline{u}$ using slip conditions as on the sides Iightly shaded). The normal velocity $\underline{n} \cdot \underline{u}$ is imposed by the prescribed history of upheaval/subsidence and compression/extension.

cients of the (assumed) isotropic rocks are computed using Berryman's composite medium theory (Berryman, 1980, 1986, 1992). The shear and bulk viscosities are assumed to depend on grain contact areas and mineralogy, fitting specific formulae to geologic and experimental data from a series of test basins. Thus, the mechanical and diagenetic modifications of texture directly affect rheology which, then, is used to compute basin stress/deformation history using our extended incremental stress rheology.

The particular boundary conditions that are used to completely pose the rheologic problem are illustrated in Fig. 2. These conditions enforce the lateral compression/extension and subsidence/ upheaval imposed by the larger-scale tectonics. The interaction of the top of the sediment pile with the overlying fluids (atmosphere or sea bottom) is accounted for by the value of normal stress and the (assumed) absence of tangential shear. The no vertical-shear lateral boundary condition allows for natural compaction at the sides of the basin.

Nonlinearity and coupling are key reasons for constructing a comprehensive basin model. The modern theory of nonlinear dynamical systems has revealed their great potential for supporting a host of phenomena that arise autonomously, i.e., with- 
out their imposition by an external template. Nonlinear systems can, for example, oscillate periodically or chaotically in time and may organize spatially in regular spatial patterns (see Ortoleva, 1987a, 1987b, 1990, 1994a; Nicolis and Nicolis, 1987; Turcote, 1992).

A necessary condition that such autonomous spatio-temporal organization can take place is that the system be maintained sufficiently far from equilibrium (Nicolis and Prigogine, 1977). The potential importance of nonlinear dynamics in geological systems has been the subject of several conferences (Nicolis and Nicolis, 1987; Ortoleva et al., 1990a) and has been investigated extensively in the context of geochemistry (Ortoleva, 1994a). For the sedimentary basin, it has been pointed out (Ortoleva, 1993, 1994a,b,c; Maxwell, 1997) that nonlinear dynamics can play an important role on a wide range of spatial scales. This potential autonomy of behavior suggests that many patterns of mineralization, petroleum reservoirs, fault motion and other phenomena cannot be understood as direct consequences of related patterns of sedimentology, volcanism or tectonism, i.e., cannot be attributed to an external template. Owing to the large network of processes underlying basin dynamics, as well as the nonlinearity of the conservation equations, a computational modeling approach is likely the only way to delineate nonlinear basin phenomena and the range of conditions (overall tectonics, sedimentation history, etc.) for which they occur.

Far-from-equilibrium conditions, necessary for the operation of autonomous spatio-temporal patterning, can be obtained in a sedimentary basin. The basin is sustained out of equilibrium by the fluxes and forces applied to the boundary of the basin. Input of sediment presents the basin with minerals and fluid chemical species which, after burial, are out of equilibrium at the local pressure and temperature or other conditions. Changes in tectonic forces, heat flux or influxed magmatic or meteoric fluids can also cause all or part of the basin to be driven out of equilibrium. Other factors are the drive of the overburden towards compaction and buoyancy drive of low density fluids (oil, gas or hot aqueous liquid) to rise. These factors that drive the basin out of equilibrium are directly or indirectly imposed at the basin boundaries.
Thus, as suggested in Fig. 2, the conditions inducing change within a basin are expressed in terms of the boundary conditions imposed on the solution of the conservation equations. Clearly, rapid burial, large geothermal gradients or large amounts of chemically unstable kerogen are examples of factors favoring an increase in the likelihood of autonomous basin behavior. The comprehensive, three-dimensional, fully coupled model presented here is designed to capture this richness in autonomous basin behavior.

In this study, we use the updated Lagrangian approach to analyze the time dependent large deformation behavior of geological materials with the incremental stress rheologic behavior (Bathe et al., 1975; Synder and Bathe, 1981; Bathe, 1996). In our numerical approach, all variables are referred to an updated configuration in each time step. The approach has two major steps. First, the incremental stress rheology is solved at the integration points of the finite elements. Second, the displacements are computed by using a global deformation solver. Iterations of these two steps are performed until the norm of the change in displacements between two consecutive iterations is less than a specific tolerance. The two-step solution technique allows the introduction of new deformation mechanisms with only minor changes in the code.

The finite element grid accretes with sediment infilling. A new sediment layer is introduced when the sediment layer at the top of the basin reaches a critical thickness. In contrast, when erosion creates a top layer that is locally too thin, the finite element grid is locally reorganized to preserve numerical accuracy. This accreting, reorganizing grid that also adapts to sedimentary features as they are added (i.e., to capture thin beds) is required to capture sedimentary detail and ensure numerical stability and accuracy.

We use the conjugate gradient iterative technique with simple diagonal preconditioner to solve for the incremental displacements. The finite element code and iterative solver are parallelized.

In the following sections, the incremental stress rheology is introduced. Then, the explicit formulation of the rate of strain is provided for poroelasticity, irreversible nonlinear viscosity, pressure solution, and fracturing. The numerical solution technique 
and finite element formulation are provided in the Appendix. One- and three-dimensional simulations of the Piceance Basin (Colorado) and the Andector Field, Permian Basin (West Texas) are used to illustrate the capabilities of our approach.

\section{Incremental stress rheology}

The strongly coupled nature of the basin deformation problem is captured using an incremental stress rheology. Let us make some of the coupling explicit. The poroelasticity rate of strain $\underline{\underline{\dot{\epsilon}}}^{(p e)}$ ) may be expressed in terms of total stress $\underline{\underline{\sigma}}$, wetting phase fluid pressure $p$, and rock texture $\Theta$ via:

$\underline{\underline{\dot{\epsilon}}}^{(p e)}=\underline{D}^{-1}(\Theta) \frac{D}{D t}(\underline{\underline{\sigma}}+\alpha(\Theta) p \underline{\underline{I}})$

for fourth rank tensor of poroelastic coefficients $D$ and effective stress coefficient $\alpha ; D / D t$ represents a material time derivative measuring the rate of change of a tensor in time with respect to a local reference frame fixed to a translation, rotating material point. The texture $\Theta$ represents a set of variables characterizing the mineralogy, shape, size, orientation, and packing of the grains, and also the fracture length, aperture, number density and orientation statistics. In summary:

$\underline{\underline{\dot{\epsilon}}}^{(p e)}=\underline{\underline{\dot{\epsilon}}}^{(p e)}(\Theta, p, \underline{\underline{\sigma}})$,

illustrates the strong coupling among deformation, fluid properties and texture.

A direct coupling of mechanics and chemistry arises through pressure solution. Grain dissolution at stressed grain-grain contacts induces compaction and thereby contributes to $\dot{\epsilon}$. The rate of this pressure solution contribution, $\underline{\underline{\dot{\epsilon}}}^{(p s)}$, depends on the stress at grain-grain contacts and, hence, on the macro-stress $\sigma$, fluid pressure and texture. However, $\underline{\underline{\epsilon}}^{(p s)}$ should also depend on the composition of the pore fluid. The latter may be characterized by the set of concentrations $c=\left\{c_{1}, c_{2}, \ldots c_{N}\right\}$ for the $N$ pore fluid species system; hence $\underline{\underline{\dot{\epsilon}}}^{(p s)}$ depends on $\sigma, \Theta, p$ and $c$.

As the present theory is macroscopic, the variables describing fractures (length, aperture, number density and orientation statistics) are considered to be part of the texture $\Theta$ (Tuncay et al.,
2000). This assumes that the length scale on which the phenomena of interest vary is much greater than the fracture length or inter-fracture spacing. Otherwise one must treat fractures individually, an approach that is not viable for basin-scale modeling. With our macro-textural description, one must allow for the potential influence of the fracture variables on rock mechanical and other properties.

One expects that $\underline{\underline{\epsilon}}^{(j)}$ (where $j=$ poroelasticity, viscosity, pressure solution, fracturing, etc.) should, in general, depend on all the aforementioned variables $(\underline{\sigma}, \Theta, p, c)$, as well as absolute temperature $T$. With this:

$\underline{\underline{\dot{\epsilon}}}=\sum_{j=1}^{N_{d}} \underline{\underline{\dot{\epsilon}}}^{(j)}(\Theta, \underline{\underline{\sigma}}, p, c, T)$

The dependency of $\underline{\underline{\epsilon}}^{(j)}$ on the indicated state variables may be nonlocal in time. For example, in the case of poroelasticity, $\underline{\underline{\epsilon}}^{(p e)}$ depends on the time-derivative of effective stress (see Eq. (2)). Therefore, the $\underline{\underline{\epsilon}}^{(j)}$ may be functionals of their arguments that can, in principle, sample the state variables in some finite volume of space-time.

The total rate of strain $\underline{\underline{\epsilon}}$ is defined by:

$\dot{\epsilon}_{i i^{\prime}}=\frac{1}{2}\left(\frac{\partial u_{i}}{\partial x_{i^{\prime}}}+\frac{\partial u_{i^{\prime}}}{\partial x_{i}}\right)$.

The six independent components of the symmetric second rank tensor Eq. (4) must be supplemented with three additional equations so that the three deformation velocity components $\left(\underline{u}=u_{1}, u_{2}, u_{3}\right)$ can be determined. The required condition arises from force balance:

$\sum_{i^{\prime}=1}^{3} \frac{\partial \sigma_{i i^{\prime}}}{\partial x_{i^{\prime}}}+f_{i}=0$

for body force $f_{i}$ which, for gravity, is given by:

$f_{i}=g \rho_{m} \delta_{i 3}$.

Here $g$ is the gravitational acceleration, $\rho_{m}$ is the mass density, and the 3-direction is upward.

The above formulation must be augmented with equations of texture dynamics and fluid mass and energy conservation (the latter to fix $T$ ). With this, the model provides a complete theory of basin dynamics when the equations are solved using the 
boundary conditions imposed by the overall tectonics and by the surfacial fluids (i.e., ocean bottom and atmospheric pressure) (Figs. 1, 2).

Effects such as strain hardening or weakening are accounted for in the present model via the coupled dynamics of texture and stress. The differential equations of texture evolution introduced the time delays (memory) that make our rheology capture hardening or softening. The latter properties are reflections of texture, i.e., hardness/ weakness is a unique function of texture but not of stress. Thus, rock rheology depends on texture which, via the evolution equations of the latter, depends on the history of deformation.

To complete the incremental stress formulation, explicit expressions for the rate functions $\underline{\underline{\dot{\epsilon}}}^{(j)}(\Theta, \underline{\underline{\sigma}}, p, c, T)$ are required. For $\underline{\underline{\dot{\epsilon}}}^{(p s)}$, for example, these can be obtained through geometric considerations of the texture variables and the rate of grain shortening from pressure solution (see Dewers and Ortoleva, 1994 and Ortoleva, 1994a, 1998).

The dependence of the strain rates on state clarifies the central role of incremental stress theory in integrating all the RTM basin processes into a unified model. It is the coupling allowed by this integration that underlies many key basin phenomena from fault dynamics to episodic fluid flow, seal formation and overpressure.

In addition to the coupling of deformation to other phenomena through the incremental stress formulation, there are numerous indirect couplings. For example, rock properties such as permeabilities, reactive grain surface area and thermal conductivities depend strongly on texture. As the latter is affected by stress and deformation, a complex network of coupling relations is thereby expressed. For further discussion of the consequence of this network, see Ortoleva et al. (1987a, 1987b), Ortoleva (1994a, 1994b, 1994c, 1998), and Dewers and Ortoleva (1994).

\section{Explicit formulation of the $\underline{\underline{\epsilon}}^{(j)}$}

\subsection{Poroelasticity}

The poroelastic formulation adopted is as in Eq. (2). It is the implementation of the theory of
Biot (Biot, 1941; Gassman, 1951; Biot and Willis, 1957) modified for an incremental strain rate. When the medium is isotropic, the fourth rank elasticity tensor can be written as:

$D_{i j k l}=K \delta_{i j} \delta_{k l}+G\left(\delta_{i k} \delta_{j l}+\delta_{i l} \delta_{j k}-\frac{2}{3} \delta_{i j} \delta_{k l}\right)$

where $K$ and $G$ are bulk and shear moduli of the drained porous medium. These moduli and the effective stress coefficient of the macroscopic porous medium can be approximately calculated in terms of the grain sizes, composition, porosity, and mineral elastic properties by using Berryman's (1980, 1986) approach.

\subsection{Nonlinear viscosity and yield}

The inelastic mechanical contribution to $\dot{\underline{\epsilon}}$ is cast in the present approach as a nonlinear viscosity law in the form:

$\underline{\underline{\dot{\epsilon}}}^{(v p)}=\underline{\eta}^{-1}(\underline{\underline{\sigma}}+\tilde{\alpha} p \underline{I})$.

The fourth rank viscosity tensor $\underline{\eta}$ depends on stress, fluid pressure and texture. The second term in the effective stress involves a coefficient $\tilde{\alpha}$ that is usually taken to be unity. The viscosity tensor is assumed to be isotropic:

$\eta_{i j k l}=\kappa \delta_{i j} \delta_{k l}+\mu\left(\delta_{i k} \delta_{j l}+\delta_{i l} \delta_{j k}-\frac{2}{3} \delta_{i j} \delta_{k l}\right)$

for shear and bulk viscosities $\mu$ and $\kappa$, respectively. To capture faulting, shear viscosity is assumed to depend on a yield function $F$. In the present work, we adopt the transitional form:

$\frac{1}{\mu}=\frac{1}{\mu_{-}}+\left[\frac{1}{\mu_{+}}-\frac{1}{\mu_{-}}\right] \frac{1}{1+\exp [-(F+w) / \Delta]}$

where $\mu_{-}$and $\mu_{+}$are the viscosities before $(F<0)$ and after $(F>0)$ yield, respectively, and $w=\Delta \ln \left(\mu_{-} / \mu_{+}\right)$such that $\mu=0.5\left(\mu_{-}+\mu_{+}\right)$for $F=$ 0 . The width of the yield transition $\Delta$, like $\kappa, \mu_{+}$ and $\mu_{-}$, depends on $\Theta$ (and possibly on other state variables, notably $\sigma, p, T$ and $c$ ).

The texture dependence of the viscosities is not 
well characterized. We conjecture that the area of grain-grain contact should strongly affect the viscosities. Assume that the greater the free face area relative to grain-grain contact area, the lower the viscosity. For a monomineralic system, let $x_{f}$ be the fraction of a grain surface that is free (and not in contact with other grains). Then consider the assumption:

$$
\mu_{-}=\mu_{-}^{0} \frac{1-x_{f} / x_{f}^{*}}{1+\left(x_{f} / x_{f}^{0}\right)^{\gamma}}
$$

for parameters $x_{f}^{*}, x_{f}^{0}$, and $\mu_{-}^{0}$ that are particular for each mineral. Note $\mu_{-}^{0}$ is the viscosity for the zero porosity, monomineralic rock. An alternative approach is the approximation of viscosities as a function of grain size and porosity. For example:

$\mu_{-}=A \exp \left(-\phi / B \phi^{0}\right)^{n} g(r)$

where $A, B$, and $n$ are material properties, $g(r)$ is a correction for grain size $r$, and $\phi$ is porosity.

For the $M$ mineral system $(i=1,2, \ldots M)$, consider the mode (solid volume fraction) average:

$\eta_{-}^{-1}=\frac{1}{1-\phi} \sum_{i=1}^{M} \phi_{i} \eta_{-i}^{-1}$

Here $\eta_{-\mathrm{i}}$ is $\eta_{-}$for a pure mineral $i$ rock and $\phi_{i}$ is the fraction of rock volume occupied by mineral $i$. Note that:

$$
1-\phi=\sum_{i=1}^{M} \phi_{i}
$$

The yield function $F$ is assumed to take the form (Drucker-Prager and Prager, 1952):

$$
F=a J_{1}+b \sqrt{J_{2}}-c
$$

where $J_{1}$ is the first invariant of the effective stress tensor and $J_{2}$ is the second invariant of the deviatoric effective stress tensor. The dependence of the $a, b, c$ coefficients on mineralogy and texture has been fit with experimental data (Ozkan and Ortoleva, 1999a).

\subsection{Pressure solution}

Attempting to formulate an expression for the contribution to $\dot{\underline{\epsilon}}$ due to pressure solution requires a careful delineation of the grain-scale deformation processes. These include:

- dissolution at grain-grain contacts;

- granulation at grain-grain contacts;

- grain boundary slip;

- plastic deformation of single grains; and

- grain breakage.

In general, several or all of these microscopic processes are acting simultaneously and may be strongly interacting. It thus may be appropriate to further refine our incremental stress rheology such that $\underline{\underline{\dot{\epsilon}}}^{(v p)}+\underline{\underline{\dot{\epsilon}}}^{(p s)}$ is written as a sum of the aforementioned processes. In the present work, we shall limit our treatment to only include an explicit accounting of pressure solution via simple texture geometric models wherein the geometry of packing does not change its character due to nonpressure solution processes and all the other irreversible processes are assumed to be captured by $\underline{\underline{\dot{\epsilon}}}^{(v p)}$ as introduced above. Limitations to such a model include the neglect of gouge and a lack of a clearly defined accounting of the other nonpressure solution processes. Progress in the development of more refined models will be the subject of other studies.

Pressure solution-derived rates of strain must be formulated through a relation between the rate of change of variables characterizing the texture and through the geometric relation between texture and macroscopic strain. Such formulations have been presented elsewhere for simple texture models (Ortoleva, 1998). Their model does account for some of the interplay of mechanical and pressure solution deformation by allowing grain geometry and packing to depend on porosity, the latter being the aggregate effect of all strain processes.

The simplest pressure solution models are isogeometric. For them, the symmetry of grain packing is assumed to be preserved as pressure solution tends to dissolve minerals at grain-grain contacts that bear normal stress in excess of fluid pressure. For a single stress-supporting mineral system considered to be a periodic array of truncated spheres, 
the formula (Dewers and Ortoleva, 1990, 1994):

$\dot{\epsilon}_{33}^{(p s)}=\frac{G_{3}}{L_{3}}$

was used, where $G_{3}$ is the rate of grain shortening in the vertical (3) direction from pressure solution and $L_{3}$ is the grain height. In that formulation, the grains are described in terms of $L_{3}$, two horizontal truncation lengths $L_{1}$ and $L_{2}$, and the sphere radius $L_{4}$. Porosity can be expressed in terms of the $L$ values. The rate $G_{3}$ depends on the normal stress $P_{3}$ to the horizontal grain-grain contacts. To obtain a complete theory, this quantity must be related to the macroscopic stress $\underline{\underline{\sigma}}$ operating on a volume element containing many grains and on the texture $\Theta=\left\{L_{1}, L_{2}, L_{3}, L_{4}\right\}$. This relation has been taken to be in terms of the surface area average:

$$
L_{1} L_{2} \sigma_{33}=-A_{3} P_{3}-\left(L_{1} L_{2}-A_{3}\right) p
$$

for grain-grain contact area $A_{3}(\Theta)$ and fluid pressure $p$. This formalism has been generalized to multi-mineralic problems and for random arrays of grains of a range of sizes and shapes (Ortoleva, 1998).

Chemistry enters the pressure solution rate law through the stoichiometry of the mineral dissolution reaction and the pore fluid composition $c\left(=\left\{c_{1}, c_{2}, \ldots c_{N}\right\}\right)$ of the $N$ pore fluid species in the (assumed) single fluid phase. In the case of quartz, it is found to good approximation that:

$\frac{D L_{3}}{D t}=G_{3}=k_{3}\left[c_{\mathrm{SiO}_{2}(a q)}-K\left(P_{3}\right)\right]$

for material derivative $D / D t$, rate coefficient $k_{3}$ and equilibrium constant $K$. The latter increases with $p_{2}$ due to variations of free energy with normal stress. More complex dependencies of $K$ on stress have also been considered when strain energy is taken into account. The rate coefficient $k_{3}$ depends on the properties of the water film within the grain-grain contact and, thereby, can also depend on $P_{3}, p$, and, in principle, $c$ (Renard et al., 1997, 1999). All the above quantities depend on temperature $T$, coupling the equation of mechanics, pore fluid chemistry, and hydrology to that of energy transport. In the simulations pre- sented below, the approaches of Renard et al. (1997, 1999) are used.

\subsection{Fractures}

We have developed a model of the probability for fracture length, aperture and orientation (Tuncay et al., 2000). The model predicts the evolution of this probability in response to the changing stress, fluid pressure, and rock properties as the basin changes. The fracture probability is used to compute the permeability tensor. The latter affects the direction of petroleum migration; information which is key to finding new resources. It is central to planning infill drilling spacing and likely directions for field extension. It is key to the design of horizontal wells and the optimum rate of production in stress-sensitive reservoirs. Finally, the predicted distribution of fracture network statistics across a field is a necessary input to reservoir simulators used to optimize production.

The dynamics of the fracture network in our model is based on a statistical representation. For example, consider a set of fractures of length $L$ with normal $\underline{n}$ for a three-dimensional spectrum of normal orientations. Then the rate of change for $L$ in the rock-fixed frame takes the form:

$$
\frac{d L}{d t}=R(p, \Theta, \underline{\underline{\sigma}})
$$

where the fracture extension rate $R$ depends on the normal stress $\sigma$, the wetting phase fluid pressure $p$ and the texture $\Theta$ of the surrounding rock (including fracture length and aperture). A similar equation for the fracture aperture is developed (see Tuncay et al., 2000 for further details).

Let $f^{\prime}$ be the number density of sites at which fractures may nucleate. By definition of the undeformed state, $f^{\prime}=f$ but $f^{\prime}$ can differ from $f$ due to changes in rock texture from diagenesis or mechanical processes. In the simplest case where fracture nucleation sites are not created or destroyed, $f^{\prime}$ obeys the conservation equation $\partial f^{\prime} / \partial t+\underline{\nabla} \cdot\left(f^{\prime} \underline{u}\right)=$ 0 . In a macrovolume element of volume $V$ there are $V f^{\prime}$ fracture nuclei and hence a fracture void space $V f^{\prime} \pi r^{2} a$ where $a$ and $r$ are the aperture and radius of the assumed penny-shaped fractures, 
respectively. To compute the dilatation, we focus on a fixed volume $V_{m}$ of solids and follow its change in a time $\delta t$. The volume of the unfractured rock $V_{u n f r}$ is related to $V_{m}$ and the porosity $\phi_{m}$ of the unfractured rock via $V_{u n f r}=V_{m}+\phi_{m} V_{u n f r}$. Hence, $V_{u n f r}=V_{m} /\left(l-\phi_{m}\right)$. Note that total porosity is equal to $\phi=\phi_{m}-\phi_{f r}$. The total volume $V$ of the sample of rock containing $V_{m}$ is then

$$
V=\left(1-\phi_{m}\right)^{-1} V_{m}+V \Delta
$$

where $\Delta=f^{\prime} \pi L^{2} a$. With this, the volume of rock $V(t)$ at time $t$ for fixed volume of solids $V_{m}$ (considered incompressible and not to expand thermally or react) is given by

$$
V(t)=V_{m}\left(1-\phi_{m}\right)^{-1}(1-\Delta)
$$

Noting that

$\operatorname{tr} \underline{\underline{\dot{\epsilon}}}^{(f r)}=\lim _{\delta t \rightarrow 0} \frac{V(t+\delta t)-V(t)}{V(t) \delta t}$

one obtains

$\operatorname{tr} \underline{\underline{\epsilon}}^{(f r)}=[1-\Delta]^{-1} \frac{D \Delta}{D t}$

where $D / D t$ is the material derivative, i.e., the derivative in the reference frame fixed to the solids.

The tensor character of the fracture-mediated deformation is related to the directions of each fracture through its normal $\underline{n}$ to the fracture plane. Consider the expression

$\dot{\epsilon}_{k l}^{(f r)}=[1-\Delta]^{-1} \frac{D}{D t}\left(\Delta n_{k} n_{l}\right)$.

Here $D / D t$ represents a material time derivative; however, now, it must also account for the rotation of the fracture normals as they change direction with flexure, shearing or other deformation. Note that the trace of this expression agrees with the earlier result for the dilatation. Finally, this expression agrees with simple cases wherein all fractures are parallel.

In our model, a finite (but representative) number of fracture orientations is accounted for. We use the fracture kinetics formulation of Ortoleva (1994a), and Sonnenthal and Ortoleva
(1994). However, here we replace the least compressive stress in the formulation by the stress component normal to each fracture plane. This allows calculation of fracture length and aperture for each fracture orientation. For example, if we assume that only vertical fractures can occur as for a one-dimensional problem, since the stress component normal to any vertical plane is the same because of the symmetry, an isotropic fracture network develops. In three-dimensional problems, our proposed algorithm has the power to predict a complex fracture network with preferential orientations dictated by the structure of the stress tensor.

Since the fracture network is well defined, the anisotropic fracture permeability can be calculated approximately. The anisotropic fracture permeability of a fracture network consisting of a single fracture orientation is given by

$K_{i j}^{f r}=\lambda\left(\delta_{i j}-n_{i} n_{j}\right)$

where $\underline{n}$ is the unit normal to the fracture plane and $K^{f r}$ is the fracture permeability. The parameter $\lambda$ can be approximated by

$\lambda=\beta \phi_{f r} \frac{a^{2}}{12}$.

Here $\beta$ is a factor accounting for the connectivity of fractures. For large fracture lengths and dense networks $\beta$ approaches unity whereas for small fracture lengths and low fracture densities it vanishes (Oda, 1986). Oda $(1985,1986)$ proposed that this coefficient should be a function of a dimensionless second order tensor of fracture geometry. He called this tensor the fabric tensor (Oda, 1982). A discussion of this factor can be found in later papers by Oda $(1985,1986)$. In this study, $\beta$ is taken as unity. We assume that the total fracture permeability is obtained by summation of fracture permeabilities for all orientations and statistical classes multiplied by the fracture porosity which has been proposed previously by Chen et al. (1999). It is assumed that fluid flow is slow and the disturbance at fracture intersections is negligible. Summation is inadequate when the fracture density is lower than the percolation threshold 
(Odling, 1992; Berkowitz, 1995; Bour and Davy, 1998). Another limitation is due to the surface roughness of fractures. In this study, fracture aperture is assumed to be constant in a particular fracture. The spatial distribution of fracture aperture alters the fracture permeability. Waite et al. (1998) measured water flow through a sinusoidal fracture to compare sinusoidal flow with parallel plate flow. Their experimental and numerical results showed that a sinusoidal fracture has a significantly lower permeability and for the sinusoidal geometry the effective aperture is very close to the minimum value of the normal aperture. Thomson and Brown (1991) showed that the directional nonuniformities in the fracture surface are more important than the degree of surface roughness. Therefore Eq. (25) should be viewed as a simple fracture permeability tensor to approximate dense fracture networks with relatively smooth fracture surface. Note that the fracture permeability tensor is obtained by post processing the fracture network characteristics. We refer to Tuncay et al. (2000) for further details.

\section{Illustrative basin simulations}

\subsection{Numerical simulation}

The basin deformation model described in the previous sections was implemented in three dimensions using finite element techniques. Details on our approach are presented in the Appendix. Results presented below illustrate some of the many phenomena supported by this nonlinear basin dynamical system.

\subsection{One-dimensional simulations}

\subsubsection{Comparison with an analytical solution}

To verify our numerical approach, we compare predictions with an analytical solution obtained for the compaction of a viscoelastic layer overlying a rigid bed rock. The layer is subjective to its own weight. In the absence of fracturing and pressure solution, the total rate of strain tensor is $\underline{\underline{\underline{\epsilon}}}=\underline{\underline{\dot{\epsilon}}}^{p e}+\underline{\underline{\dot{\epsilon}}}^{v p}$ where $\underline{\underline{\dot{\epsilon}}}^{p e}$ and $\underline{\underline{\dot{\epsilon}}}^{v p}$ are given by Eqs. (2) and (9). To obtain the analytical solution we assume that elastic and viscous properties are constant and deformations are small. Letting $z$ represent vertical distance measured from the surface (downward), the lateral stress, vertical stress and displacement are obtained in the form:

$$
\begin{aligned}
\sigma_{z z}= & -\rho g z \\
\sigma_{y y}= & -\frac{b}{b+c} \sigma_{z z}+\left(\frac{e}{d}+\frac{b}{b+c}\right) \sigma_{z z} \mathrm{e}^{-(b+c)(d-e)(2 e+d) t / d} \\
w_{3}= & \frac{\rho g\left(h^{2}-z^{2}\right)}{2 d}\left[\frac{(c+2 b)(c-b)}{c+b} d t\right. \\
& -\frac{2 a^{2}}{(c+b)^{2}(d-e)(d+2 e)} \mathrm{e}^{-(b+c)(d-e)(d+2 e) t / d} \\
& \left.+1+\frac{2 a^{2}}{(c+b)^{2}(d-e)(d+2 e)}\right]
\end{aligned}
$$

where

$a=c e+d b+b e$

$$
\begin{aligned}
& b=\frac{2 \kappa+2 \mu / 3}{(\kappa+4 \mu / 3)(2 \kappa+2 \mu / 3)-2(\kappa-2 \mu / 3)^{2}} \\
& c=\frac{\kappa-2 \mu / 3}{2 \kappa+2 \mu / 3} b \\
& d=\frac{(1-v) E}{(1+v)(1-2 v)} \\
& e=\frac{v}{1-v} d .
\end{aligned}
$$

We take the model parameter values as $h=$ $0.5 \mathrm{~km}$, Poisson's ratio $v=0.3$, elasticity modulus $e=10000 \mathrm{MPa}, \kappa=\mu=10^{10} \mathrm{MPa} \mathrm{s}, \rho=2200 \mathrm{~kg} / \mathrm{m}^{3}$. The analytical and numerical results for the displacement $\mathrm{w}_{3}$ of the surface as a function of time are illustrated in Fig. 3. The match between the analytical and numerical results is excellent. 


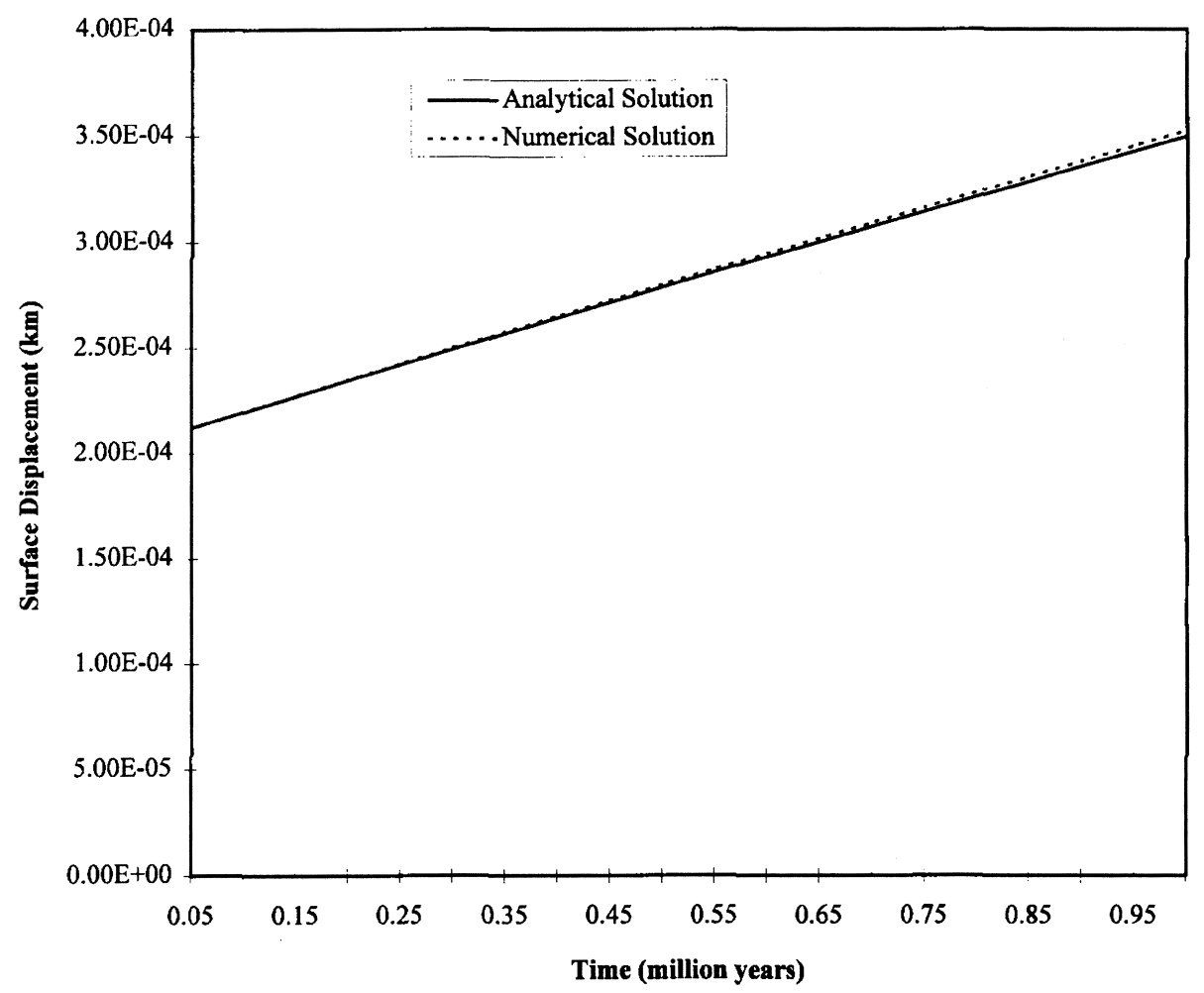

Fig. 3. Comparison of numerical and analytical results for the surface displacement.

\subsubsection{Analytical solution for least compressive} stress of a subsiding overpressured viscoelastic medium

An analytical solution is obtained for the least compressive stress of a subsiding, overpressured viscoelastic rock to illustrate some of the interesting aspects of our incremental stress approach. We assume that the vertical stress and pressure are given by:

$\sigma_{z z}=\sigma_{z z}^{0}+A_{3} t$

$P=P^{0}+A_{1} t+A_{4} \cos \left(A_{2} t\right)$

where the superscript $o$ refers to initial values, and the parameters $A_{i}$ are constants. Then the lateral stress is obtained in the form:

$$
\begin{aligned}
\sigma_{z z}= & \left(\sigma_{z z}^{0}-D_{1}-D_{3}\right) \exp \left(-\frac{c_{11}+c_{12}}{d_{11}+d_{12}} t\right) \\
& +D_{1}+D_{2} t+D_{3} \cos \left(A_{2} t\right)+D_{4} \sin \left(A_{2} t\right)
\end{aligned}
$$

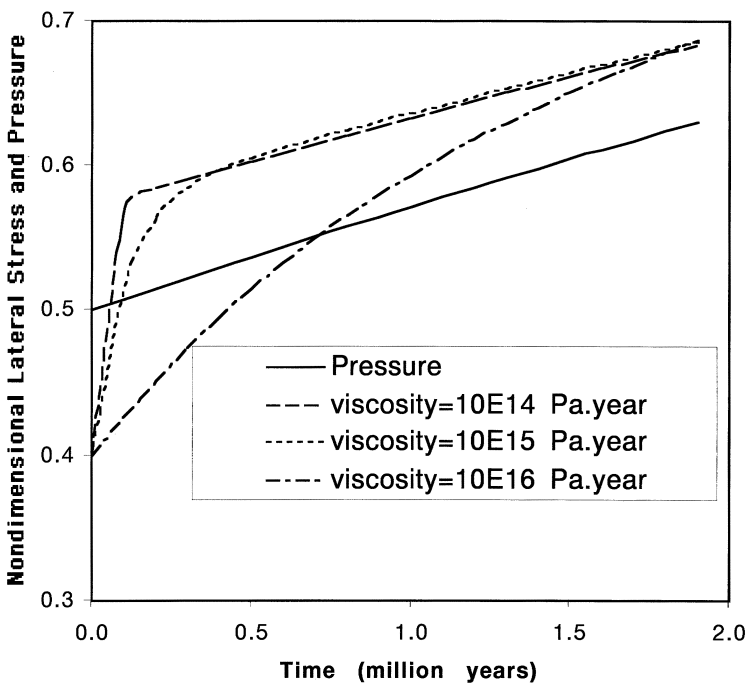

Fig. 4. History of lateral stress and pressure (Eqs. (29) and (11)). When the ratio of viscosities is kept constant $(R=1)$, the lateral stress exceeds pressure in less than one million years even for high viscosities. 
where

$$
\begin{aligned}
\sigma_{x x}^{0}= & -\frac{d_{12}}{d_{11}+d_{12}}\left(\sigma_{z z}^{0}+\alpha P^{0}\right)-\alpha P^{0} \\
d_{11}= & (2 K+2 G / 3) /\left[(K+4 G / 3)^{2}\right. \\
& \left.+(K+4 G / 3)(K-2 G / 3)-2(K-2 G / 3)^{2}\right] \\
d_{12}= & -\frac{K-2 G / 3}{2 K+2 G / 3} d_{11} \\
c_{11}= & (2 \kappa+2 \mu / 3) /\left[(\kappa+4 \mu / 3)^{2}\right. \\
& \left.+(\kappa+4 \mu / 3)(\kappa-2 \mu / 3)-2(\kappa-2 \mu / 3)^{2}\right] \\
c_{12}= & \frac{\kappa+2 \mu / 3}{2 \kappa+2 \mu / 3} c_{11} \\
Z=- & \left(d_{11}+d_{12}\right)^{2} A_{2}^{2}-\left(c_{11}+c_{12}\right)^{2} \\
D_{1}= & {\left[-\left(d_{11}+2 d_{12}\right) \alpha A_{1}+\left(c_{11}+2 c_{12}\right) P^{0}\right.} \\
& \left.-d_{12} A_{3}+c_{13} \sigma_{z z}^{0}-\left(d_{11}+d_{12}\right) D_{2}\right] /-\left(c_{11}+c_{12}\right) \\
D_{2}= & {\left[\left(c_{11}+2 c_{12}\right) A_{1}+c_{12} A_{3}\right] /-\left(c_{11}+c_{12}\right) } \\
D_{3}= & {\left[-\left(d_{11}+d_{12}\right)\left(c_{11}+2 c_{12}\right) A_{2} A_{4}\right.} \\
& \left.+\left(d_{11}+2 d_{12}\right)\left(c_{11}+c_{12}\right) \alpha A_{2} A_{4}\right] / Z \\
D_{4}= & {\left[\left(d_{11}+2 d_{12}\right)\left(d_{11}+d_{12}\right) \alpha A_{2}^{2} A_{4}\right.} \\
& \left.+\left(c_{11}+c_{12}\right)\left(c_{11}+2 c_{12}\right) A_{2}\right] / Z . \\
&
\end{aligned}
$$

In obtaining this result it was assumed that the material properties do not change in time, and deformations are small.

We take coefficients $A_{1}, A_{2}, A_{3}$, and $A_{4}$ as -0.1 , $0,0.05$, and 0 , respectively. The bulk and shear moduli are taken as $K=G=10 \mathrm{GPa}$. In Fig. 4 , the variation of pressure and lateral stress is shown for three different viscosities keeping the shear viscosity/bulk viscosity ratio $R$ as one. As seen in the figure, depending on the magnitude of viscosity, the lateral stress eventually catches up to the fluid pressure. This rock will hardly fracture in a one-dimensional system unless there is a high rate of overpressuring such as from petroleum generation. Even for the high viscosity rock, the lateral stress reaches the fluid pressure in less than one million years.

The effect of the viscosity ratio $R$ is illustrated in Fig. 5 where the coefficients $A_{i}$ are taken as
$-0.1,10,0.05$, and 0.1 , respectively. In this figure, we keep the bulk viscosity as $10^{15} \mathrm{~Pa}$ year, and vary the shear viscosity of $R=0.5$ and $R=1$. For $R=0.5$, rock fractures at very high pressures whereas for $R=1$, rock fractures a number of times even at low pressures. This example shows that a simple criterion, such as assumption of fracturing when fluid pressure exceeds a certain fraction of lithostatic stress, is not valid for viscoelastic media.

The physics of stress evolution is complicated since bulk and shear viscosities are dictated by the evolving microscale rock texture. Concepts such as seals (low permeability zones) emerge but are tempered with notions of resistance to fracturing, i.e., should be of low $R$ in character. In the next section, an example of episodic fracturing of a seal rock is illustrated.

\subsubsection{Episodic oil ejection}

As discussed in the previous section, most seal rocks will not fracture in a one-dimensional system (no geometric effects such as flexure) unless there is a high-rate, overpressuring mechanism in operation. In this section, we illustrated the effect of high rate of oil generation in a source rock just

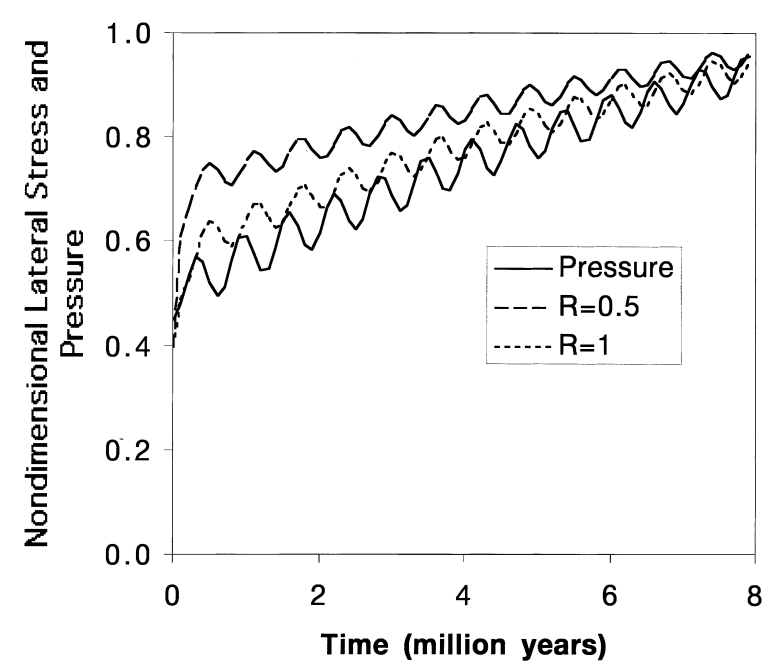

Fig. 5. History of lateral stress and pressure with harmonic pressure oscillation. For low viscosity ratio, lateral stress is higher than pressure except for very high, almost lithostatic stresses. When the viscosity ratio is high, pressure catches up with lateral stress a number of times. 
below a seal. The location of the simulated system is the Andector Field of the West Texas Permian Basin (Tuncay et al., 1998). The basin is approximately 500 million years old. The history of sedimentation rate, and mineralogic and textural character are taken from published reports as are thermal and subsidence/upheaval, erosional and thermal (i.e., basement heat flux) histories (see Tuncay et al., 1998).

Evolution of overpressure at the bottom of the Ellenburger Formation is shown in Fig. 6. Overpressuring starts around 350 million years into the simulation when fractures in the layer above the source rock first disappear but then cyclically reappear. Oscillatory behaviour is a result of cyclic fracturing and healing of the seal (Fig. 7) driven by petroleum generation. After 470 million years, the cyclic petroleum expulsion ceases and the pressure, oil saturation, fracturing and other variables show a more steady behavior.

Fig. 7 illustrates the fracture permeability/depth profile time sequence demonstrating a fracture front moving through the seal located between 2450 and $2700 \mathrm{~m}$. Overpressuring of the oil and water phases is primarily due to oil generation. It creates a fracture front that moves upward through the seal. Once the overpressure is released, the fractures close, which in turn results in descent of the fracture front and the recommencing of overpressuring. This cycle repeats until the oil generation rate slows down, or the seal remains fractured. The latter could occur due to an extremely high rate of petroleum generation or due to tectonic effects (flexure or overall extensional regime). Each fracture front cycle corresponds to one peak in Fig. 6 and to a pulse of oil release.

This type of overpressuring, fracturing, escape of fluids and the closure/healing of fractures (OFEC cycle) has been studied by a number of authors (Ghaith et al., 1990; Chen et al., 1994; Dewers and Ortoleva, 1995; Maxwell, 1997; see also discussion in Ortoleva, 1994a, 1998).

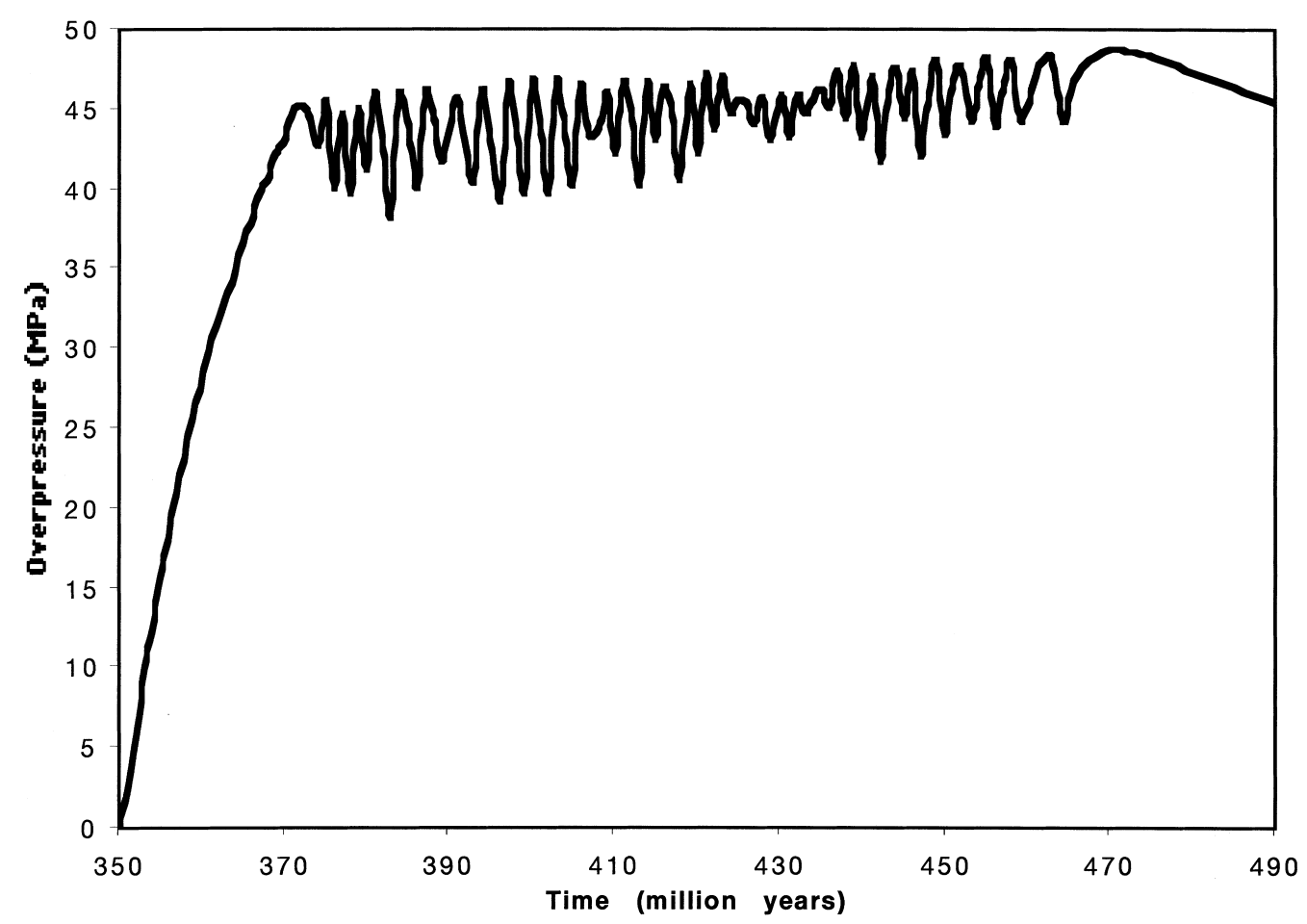

Fig. 6. Evolution of overpressure at the bottom of the Ellenburger Formation. Oscillatory behavior is a result of cyclic fracturing of the seal driven by petroleum generation. 

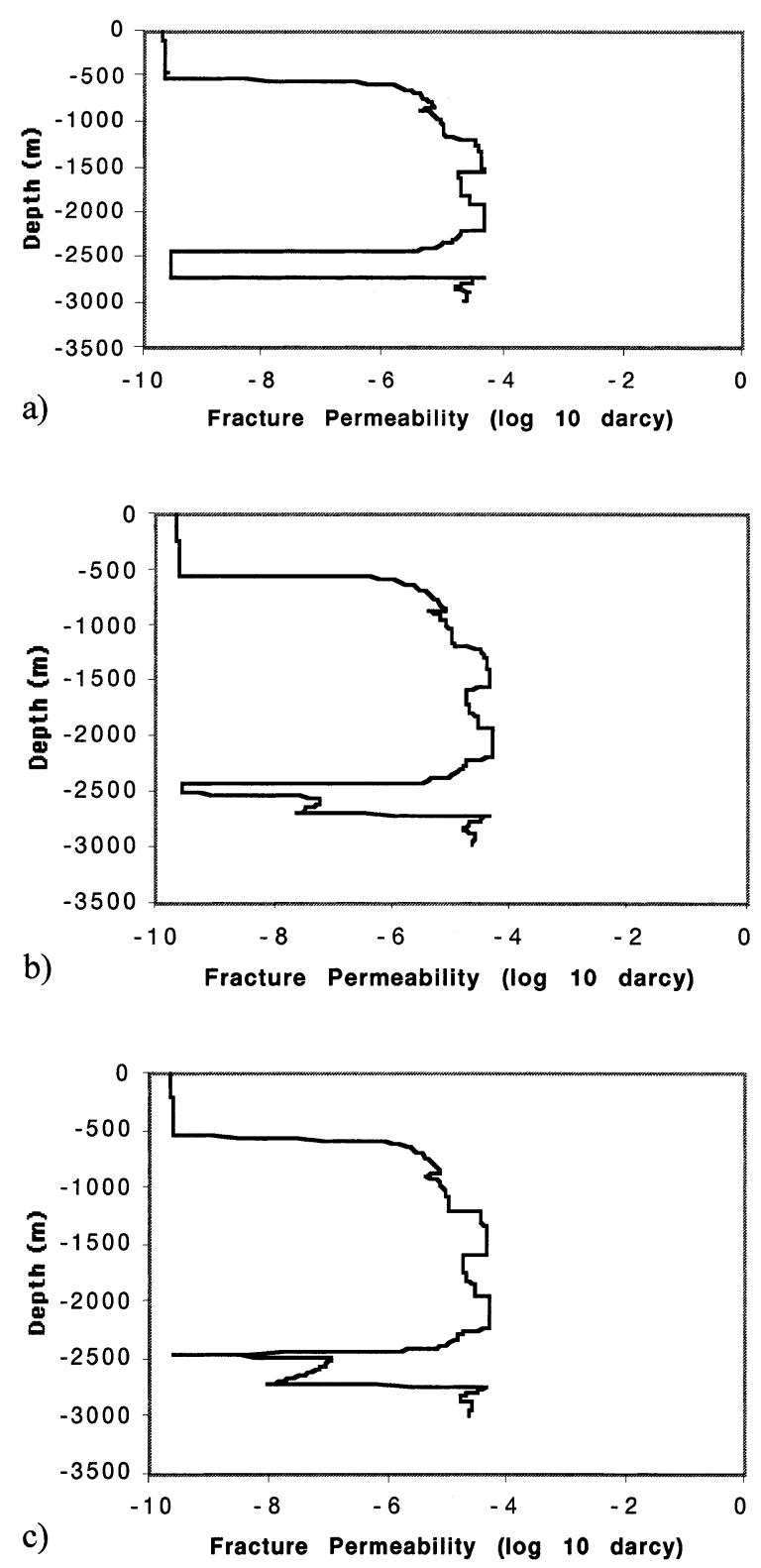

Fig. 7. Fracture permeability profile sequence illustrating the fracture front moving through the seal (between 2450 and $2700 \mathrm{~m})$.

However, these studies of the OFEC cycle do not include the self-limiting character of fracturing as accounted for in the present incremental stress formulation. The present study shows that OFEC cyclicity can still occur and its characteristic of oscillator fracture front motion is still preserved.

\subsection{Three-dimensional systems}

\subsubsection{Curvature versus permeability diagrams}

A comprehensive RTM model derives its predictive power from its basis in the physical and chemical laws that govern the behavior of geological materials. This is in contrast to correlative approaches such as porosity/depth curves or curvature analysis that attempt to use statistical average behavior. Many aspects of geological systems involve a multiplicity of factors controlling their evolution and, furthermore, there are both memory-preserving and -destroying processes. Therefore, it does not seem that there are simple correlations between today's state variables and processes that operated tens or hundreds of million years ago. Here we examine such correlations within the framework of our basin model.

Fig. 8 shows a correlation diagram for permeability with local curvature. The data are taken from a simulation performed for the Piceance Basin, Colorado (see below). The curvature has been calculated as the sum of the absolute values of the curvature tensor $\kappa_{i t}^{\prime} \equiv \frac{1}{2}\left\{\partial n_{i} / \partial x_{i^{\prime}}+\partial n_{i^{\prime}} / \partial x_{i}\right\}$ where $n_{i}$ is the upward pointing normal vector to the top of the lithologic layer in which the material point is located (at the closest point on the latter contact surface). This figure shows that prediction of fractured zones based on such a correlation is

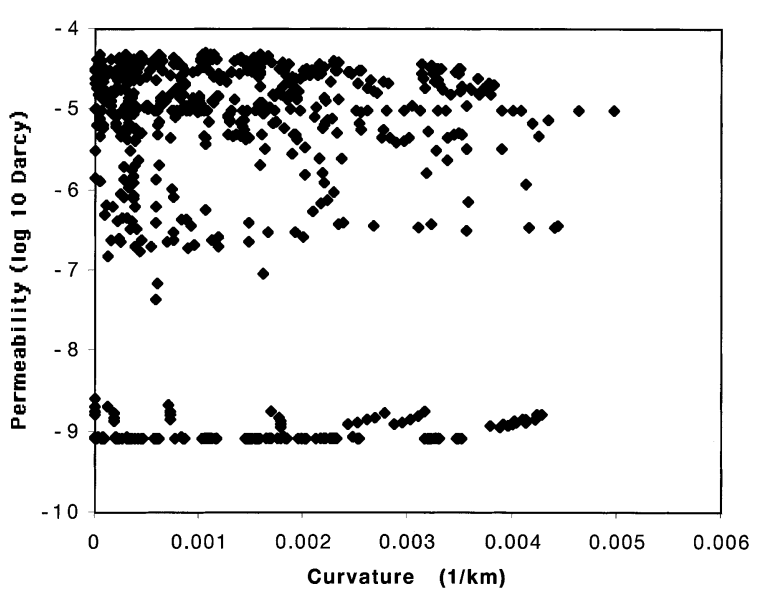

Fig. 8. Correlation of curvature with permeability shows little relation. The absolute sum of eigenvalues of the curvature tensor is used to represent curvature. 
not a reliable approach. The correlation between curvature and fracturing is very weak. Indeed, fracturing is a very dynamic process, and is affected by many parameters such as fluid pressure, tectonic boundary conditions, rock viscosities and thermal regime as well as the time course of these factors.

To better understand this effort, the time course of the state of a particular material point in the permeability/curvature plane can be studied. Fig. 9 shows the complexity of such an evolution for two material points. The first material point shows a fast compaction period followed by fracturing and healing whereas the second material point shows compaction followed by fracturing. As today's rock state is only one point on such a trajectory, it is clear that there is little hope of finding a simple correlation for such a system. Fig. 9 shows the history of permeability and curvature for two material points (relatively brittle and ductile) as the basin evolves. First, the permeability reduces as a result of compaction. The brittle rock permeability and curvature correlates as the curvature increases during an early period. However, fractures remain open as the curvature disappears due to the changing tectonics. Following such trajectories leads one to strongly argue against the reliability of present-day (i.e., snapshot) correlations.

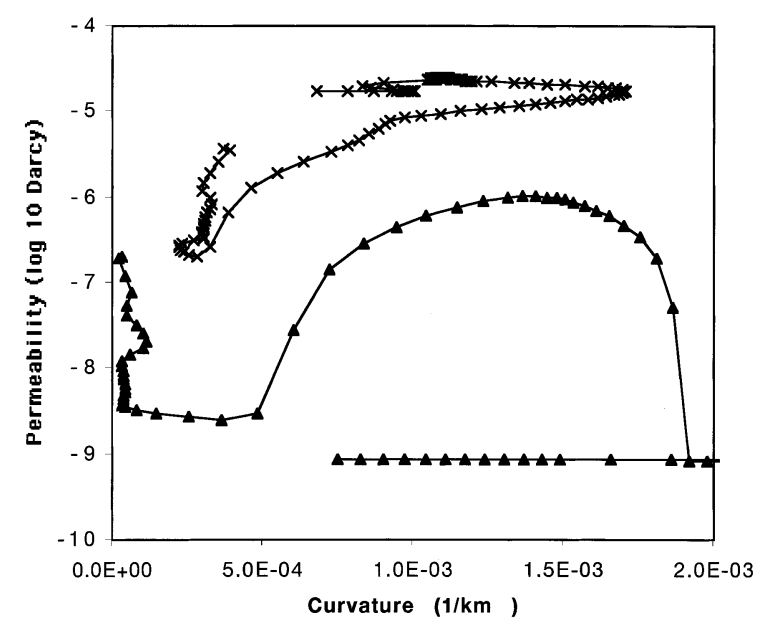

Fig. 9. Trajectory of a material point in the permeability/curvature plane. One of the material points heals whereas the other does not. The high permeability material point is in a sandstone layer.

\subsubsection{Three-dimensional deformation of the south- central zone of the Piceance Basin}

The map view of the simulation domain of this study is shown in Fig. 10. Data from five wells are used to reconstruct the sedimentation, erosion and subsidence/upheaval histories (shown in Fig. 10).

The Piceance Basin history and local site data were distilled into specific input files for the boundary conditions and initial data as follows. The depositional history input (thickness of units and ages of contacts) was constructed from well logs such that the sandstone to the mudstone ratio, as well as the total coal thickness in the Paludal Interval, are preserved. The sandstones in the Mesaverde Group were combined such that no single unit is less than $30 \mathrm{~m}$ thick, in order to satisfy the minimum thickness for the chosen resolution of the simulation grid. For simplicity, the overlying Wasatch Formation and younger units are left undifferentiated. Lithologic input (grain size distribution) for the Mesaverde Group was modified from petrographic data primarily for coarser grained units in the Mesaverde Group at the MWX site (Multiwell Experiment Project Groups at Sandia National Laboratories and CER Corporation, 1987, 1988, 1989, 1990). These data were compiled and averaged for sandstones in each interval in the Williams Fork Formation, and for each of the three major sandstone units in the Iles Formation. For the lithologic input of sandstone units, the sum of the observed average contents of quartz and feldspar minerals were assigned to the framework grains, and the observed total clay content was assigned to the clay matrix fraction. This composition is then normalized by the program to an assumed depositional porosity of $30 \%$, based on average porosities for poorly to moderately sorted, very fine to medium grained, wet-packed sands (Beard and Weyl, 1973). Due to the paucity of available data for the composition of these mudstones and shales, the input compositions for this study are loosely based on general mudstone compositional data (Shaw and Weaver, 1965) and on compositional data of the Wasatch Formation mudstones (Hosterman and Dyni, 1972). These compositional fractions are then normalized in the program to account for an assumed depositional porosity of $25 \%$. We refer to Payne 


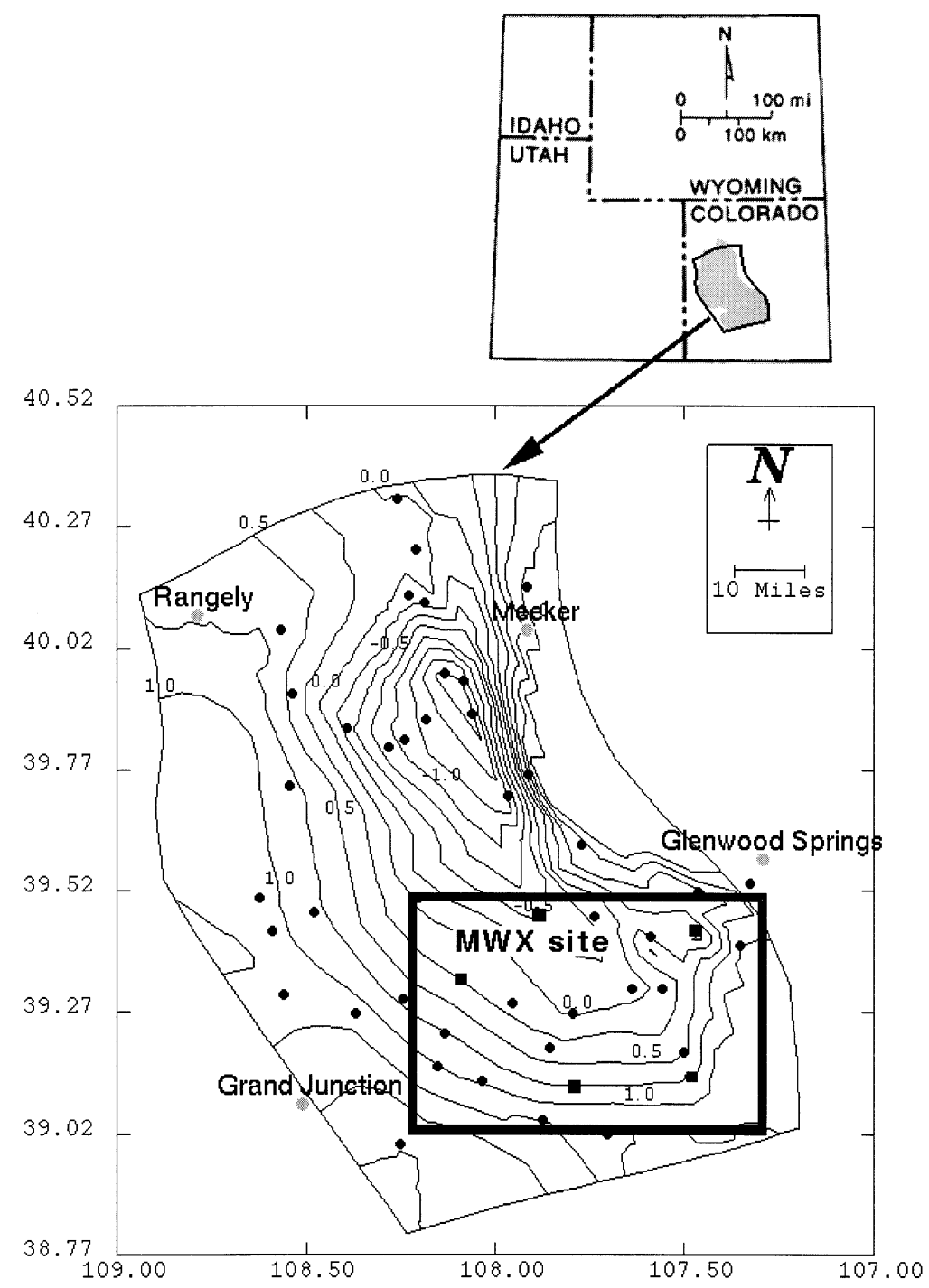

Fig. 10. Map view of the Piceance Basin and simulation domain (thick box). The small dots indicate locations of wells at which lithologic and other data are available whereas the square markers indicate locations of five wells used in the reconstruction of sedimentation and subsidence/upheaval histories.

et al. (2000) and Payne (1998) for further details on data preparation and the geologic history of the Piceance Basin.

Most of the natural gas production in the Piceance Basin is from fractured reservoirs. It is important to know the history of fracturing as well as the fractured zones today to understand the migration of petroleum from source rocks to reservoirs. Furthermore, it is interesting to delin- eate the complexity of such a three-dimensional fluid-rock system as an example of a dynamic crustal system. Thus, we simulate the 75 million year history of this basin.

The simulation starts with a flat and very thin (just a few hundred meters thick) computational domain. The subsidence rate is interpolated from the well data and used to impose a velocity of descent/upheaval at the bottom of the basin. Our 
reconstructed sedimentation and erosion rate history are used to update the thickness of the uppermost layers of the sediment. When the thickness of the uppermost layer is greater than a specified value, the upper grid is split and thus the grid grows during deposition. During extended periods of erosion, the grid is automatically reorganized so as to maintain numerical stability and accuracy. The time dependent sea level is used to impose the normal traction at the top boundary of the sediment.

Fig. 11 shows the porosity distribution in a sandstone. During early times, the basin is quite flat and porosity-depth correlation, for a given lithology, is very strong. As the basin develops, the porosity distribution is strongly affected by the tectonic boundary conditions. In the last 10 million years of simulation, the curvature at the bottom decreases. However, the porosity distribution remains fairly complicated, illustrating the effect of the detailed history on the present day porosity distribution and the lack of a simple porositydepth relationship.

Fig. 12 shows the isosurface of overpressure (15 bars) toned with depth. The back panel shows the permeability distribution. The structure of the folded, multiply layered surface is dictated by the interplay of lithological differences and fracturing, and shows the three-dimensional complexity of the continuity of overpressured zones. Thus, stacked overpressured compartments when viewed as a few pressure/depth curves yield little insight into the full three-dimensionality of the compartmentation structure.

A cross-sectional view of fracture length is shown in Fig. 13. The distribution of fracture length reflects lithologic variation and the topography imposed by the basement tectonics. The layered fracture length structure is closely related to the layering in the overpressure isosurface. While Figs. 12 and 13 illustrate the present day geometric complexity, further complexity is seen when these three-dimensional images are viewed in time sequence. These aspects of the deforming basin reflect its behavior as a nonlinear dynamical system. The layered nature of fracture zones shows the lithology dependence of fracturing, i.e., fractures in a brittle lithology discontinue at the interface of more ductile lithologies.

\section{Conclusions}

The challenge of basin modeling follows from the strongly coupled nature of the processes, the large number of operating processes and the fundamentally three-dimensionality of the problem. In the present study, an incremental stress formulation has been used to integrate the suite of basin RTM processes to understand basin deformation, fracturing, and stress history and the coevolving fluid, mineral and thermal systems. This coupled RTM system has been shown here to be capable of self-organizing compartments and oscillatory, episodic fluid flow. The present comprehensive basin modeling notion confirms that the basin is to be viewed as a self-organizing nonlinear dynamical system (Ortoleva et al., 1987a,b; Ortoleva, 1990, 1994a,b,c, 1998).

A key element of our approach is the coevolution of rock texture with macroscopic deformation. The introduction of dynamical texture variables into basin models started with the work in pressure solution where a model of grain size and shape was introduced to capture compaction, stylolites and diagenetic bedding (see Ortoleva, 1994a, 1994b for reviews). In these models, the texture $\Leftrightarrow$ deformation dynamic, models can be developed for phenomena heretofore not possible with decoupled or less comprehensive models.

Key challenges for the future of comprehensive, fully coupled, three-dimensional basin modeling are as follows:

- improve fracturing and faulting descriptions and rate laws;

- calibrate the RTM laws, notably those such as continuous irreversible deformation which operate on long time scales;

- develop more efficient numerical techniques and faster computer hardware (to attain the $>10^{6}$ finite element, $>100$ descriptive variables needed for truly comprehensive, spatially resolved simulations; and

- develop efficient methods for constructing the tectonic, thermal, and sedimentation histories directly from well logs, seismic or other "raw" data.

Calibration and validation of our model is an active research area in our laboratory at present. 

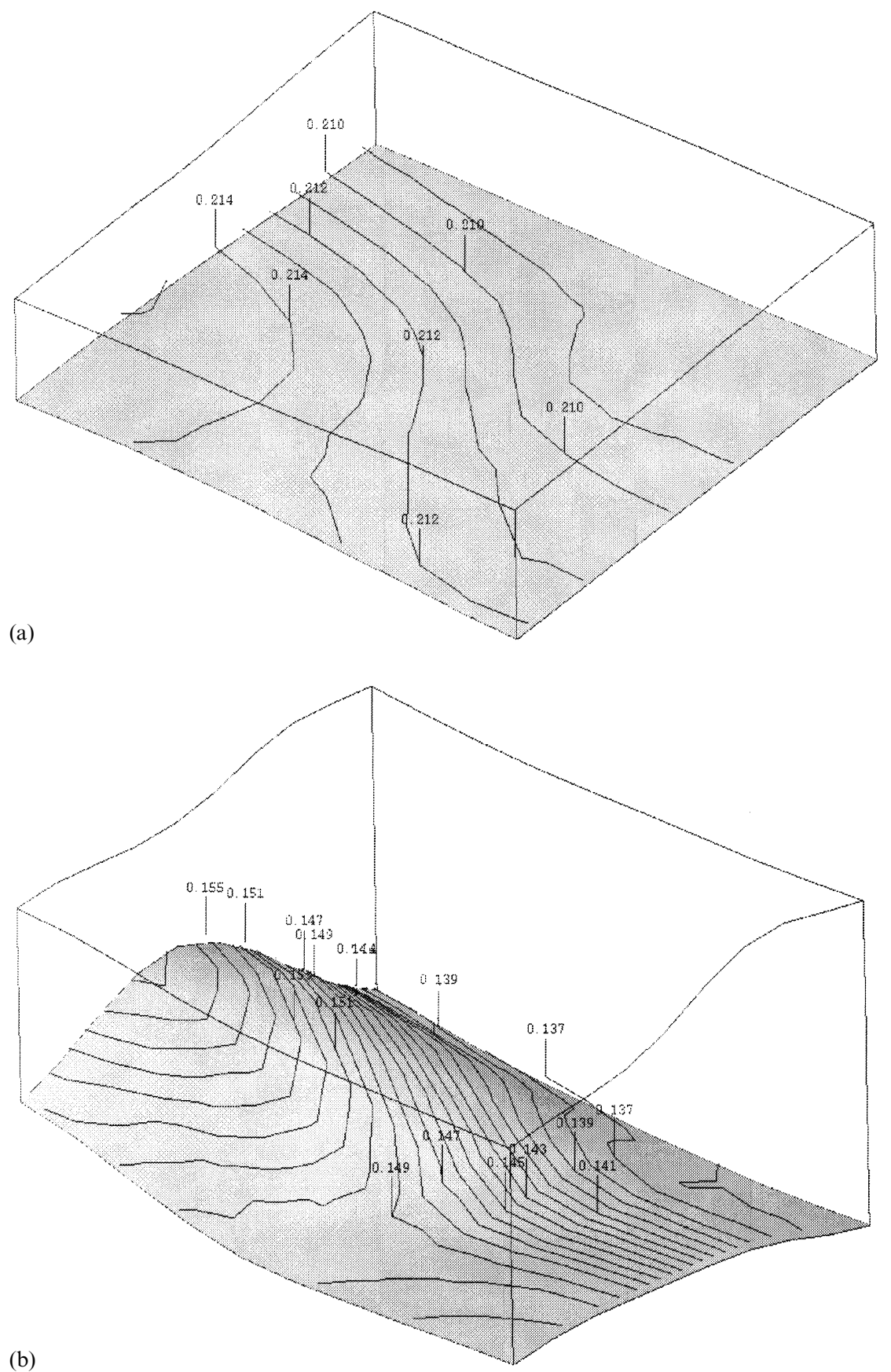

Fig. 11. Porosity contours at 20,50 and 74 (present day) million years into the simulation.

Efforts are in two directions. Instantaneous relations such as permeability/textural laws, fracture growth laws, poroelastic coefficient formulae and diagenetic rate and equilibrium parameters are being calibrated by laboratory data. Very long time processes are not accessible to laboratory 


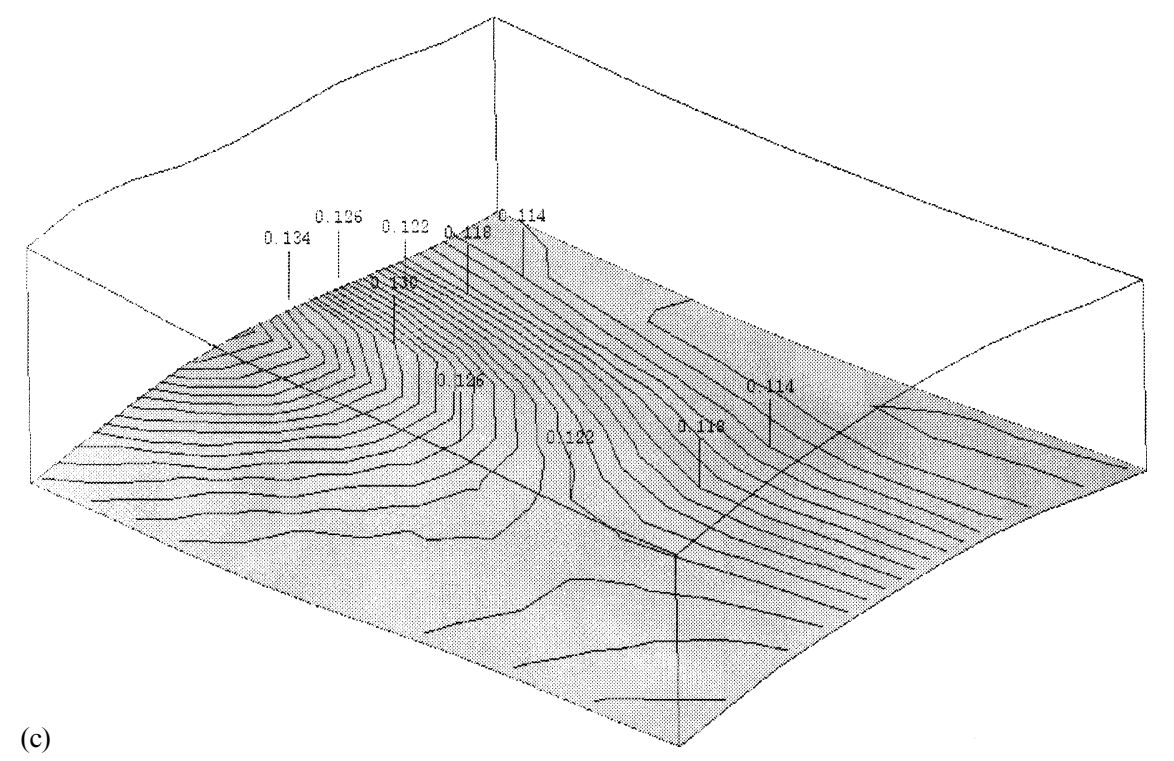

Fig. 11. (continued)

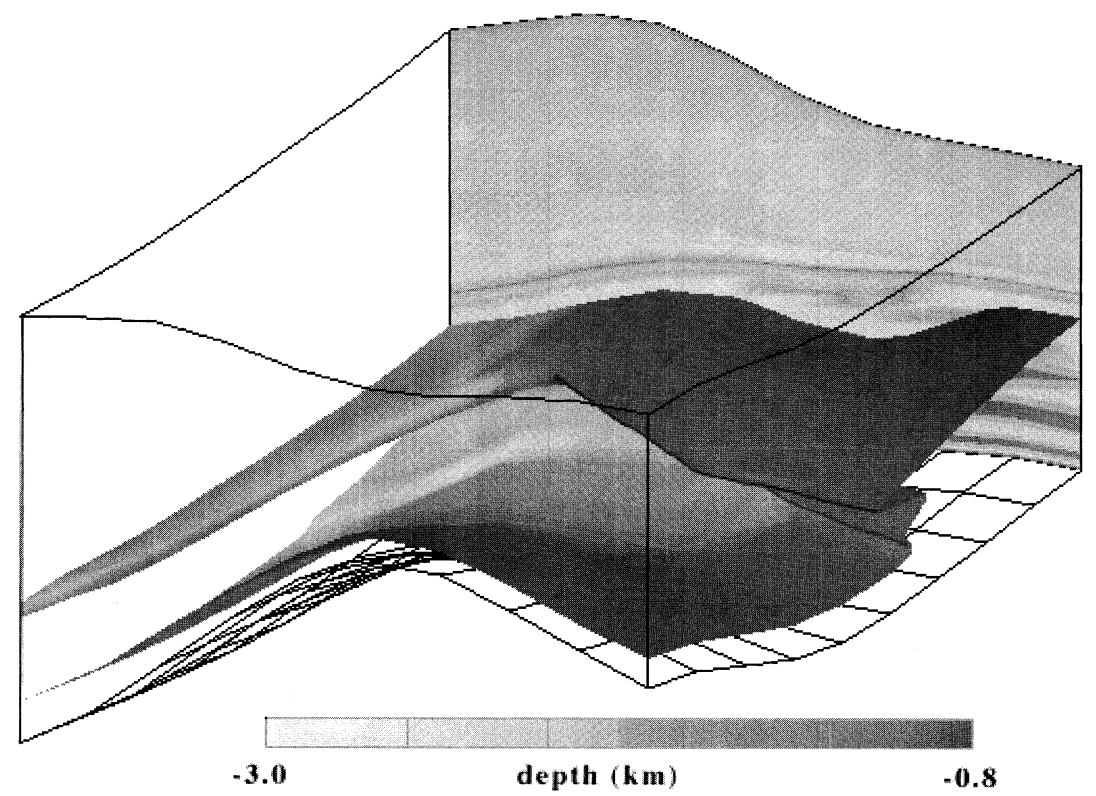

Fig. 12. Isosurface of 15 bars overpressure toned with depth. The cross-section at the back shows the permeability distribution. Different permeabilities reflect varying sediment compositions including porosity, texture and mineral grain sizes, and fractures.

experiments and are therefore being calibrated using geologic data. We have developed a "laboratory" basin database to fix these slow process parameters. This is not to be viewed as a correla- tion between overall basin properties and final basin state (such as porosity-depth curve, etc.) but rather is an approach to calibrating the physical and chemical parameters which are universal to 

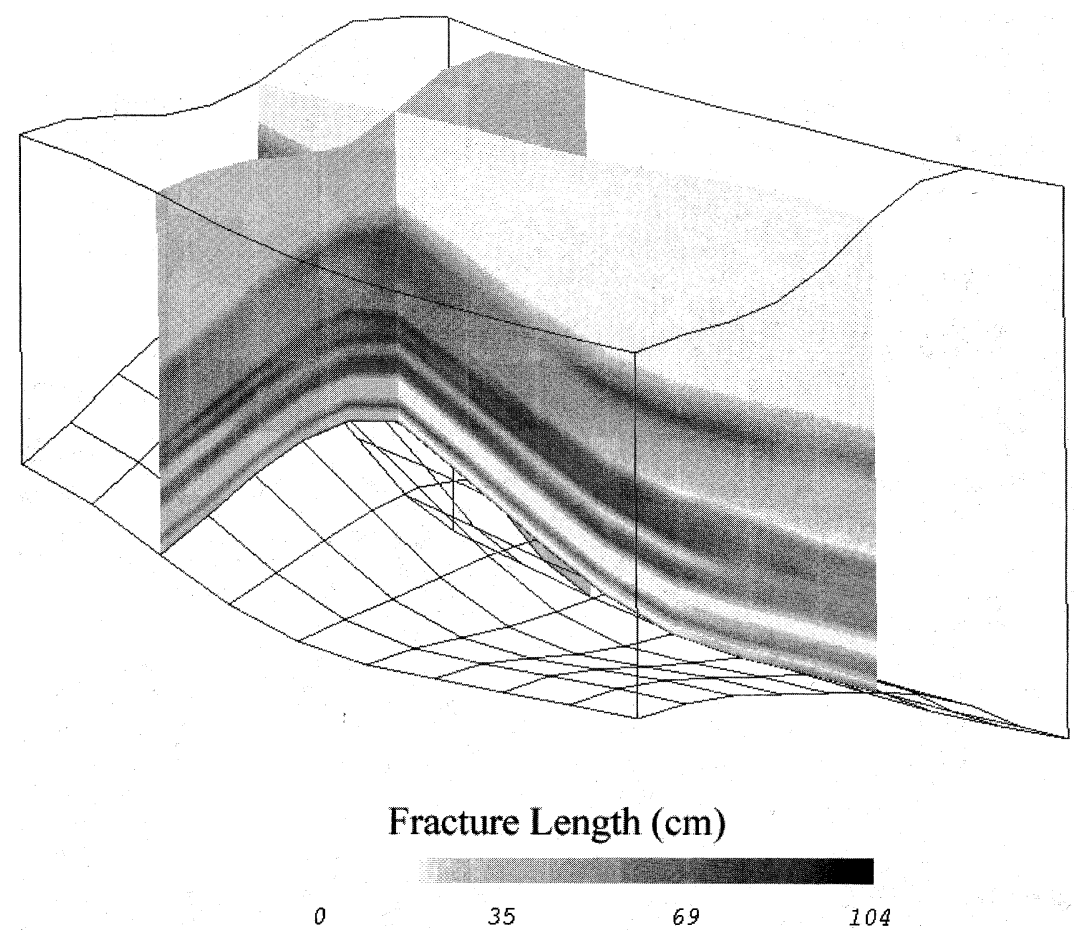

Fig. 13. Cross-sectional view of maximum fracture length illustrating the strong dependence of fracturing on lithology. Fractures discontinue at the interface of more ductile lithologies. Changing sediment properties, stress and fluid pressure during the evolution of the basin result in dynamic fracture patterns, which in turn significantly affect the anisotropy of fracture permeability. If the enhanced permeability from fracturing is significant, it can direct the flow of petroleum; understanding such occurrences of the past, therefore, can be important for identifying or understanding reservoirs in presently unlikely structural and stratigraphic locations.

all basins. With this laboratory and geological calibration approach we are also involved in validating our model on other basins not used in the calibration data set. We believe that this can be best achieved through collaborative efforts.

Our approach is of both fundamental and practical interest. Besides aiding in unraveling the dynamics of a basin, our results can be applied to: - locating quality petroleum reservoirs;

- predicting properties of the earthquake cycle and fault dynamics in general;

- determining the mechanical stability of putative chemical waste storage reservoirs/ compartments; and

- integrated management of basin water, mineral, petroleum, and related resources.

We believe that our extended incremental stress rheology provides the integration for the comprehensive, three-dimensional, RTM basin modeling needed to meet these fundamental and practical challenges.

\section{Acknowledgements}

This work has greatly benefited from conversations with and assistance from Ms. Spring Romer, Mr. Walid Sibo, Dr. Dorothy Payne and Dr. Gonca Ozkan. We thank Prof. J. Rice, Harvard University, for an introduction to the incremental stress concept. We also thank Xiaorong Luo and an anonymous reviewer whose suggestions improved the manuscript. This work was supported by a contract with the Gas Research Institute, the Office of Sciences of the U.S. Department of Energy, MEPTEC of Mobil Oil Company and Philips Petroleum Company. 


\section{Appendix: Numerical approach}

In this study we use the updated Lagrangian approach to analyze the time dependent large deformation behavior of elasto-visco-plastic geological materials. In this approach, all static and kinematic variables are referred to an updated configuration in each time step. We first obtain the integral form of the momentum balance equation. We transform the integral form to a form which allows an incremental numerical procedure.

We consider the constitutive relations in the form of:

$\left(\dot{\sigma}_{i j}+\alpha \dot{P} \delta_{i j}\right)=D_{i j k l}\left(\dot{\epsilon}_{k l}-\dot{\epsilon}_{k l}^{v p}\right)$

where $\sigma_{i f}$ is the Cauchy (real) stress tensor (force per unit area in the deformed geometry), $\dot{\epsilon}_{y}$ is the rate of deformation tensor, $D_{i j k l}$ is the fourth order elasticity tensor, $a$ is the effective stress coefficient, and over dot denotes the material derivative. For the sake of simplicity we denote all deformation processes other than poroelasticity by $\dot{\epsilon}_{i f}^{v p}$.

\section{Displacement reformulation}

It is convenient to reformulate the problem in terms of displacement defined for a small time step $\Delta t$. In that time, a material point at ${ }^{t} \underline{x}$ moves to ${ }^{t+\Delta t} \underline{x}$ through a displacement $\underline{w}$ :

${ }^{t+\Delta t} \underline{x}={ }^{t} \underline{x}+\underline{w}$

If $\underline{\sigma}$ changes continuously in time then we expect that:

$\underline{w}_{\Delta t \rightarrow 0}^{\sim} \Delta t \underline{v}$

Thus for small $\Delta t, w$ and $v$ are equivalent. In what follows, the discretized time for mutation used will be for small $\Delta t$ so that henceforth only deformation over a small time interval $\Delta t$ will be discussed. Large scale deformation will be simulated as the accumulation of small displacements over many small $\Delta t$ advancement steps.

The boundary conditions applied during the small time $(\Delta t)$ period may be written as follows. Let $S_{\phi}$ and $S_{w}$ be portions of the system boundary over which forces and displacements are specified, respectively. Then the boundary conditions to be imposed are:

$w_{i}=w_{i}^{S}=$ on $S_{w}$ and $\sigma_{i j} n_{j}=t_{i}=$ on $S_{\phi}$

where $t_{i}$ is the traction and $w_{i}^{s}$ is the specified displacement. Eqs. (A1) and (II.5) form a set of nonlinear equations in terms of the stress tensor and deformation velocities. Since the deformations are large, the system constitutes a moving boundary problem.

\section{Integral formation}

To obtain the integral form, we multiply Eq. (II.5) by an arbitrarily chosen continuous displacements such as:

$\delta w_{i}=0$ on $S_{w}$

Then we integrate the equation over the total volume $V$ :

$\int_{t+\Delta t}\left({ }^{t+\Delta t} \sigma_{i j, j}+{ }^{t+\Delta t} f_{i}\right) \delta w_{i} d v=0$

By using the divergence theorem, Eq. (A6) can be written as:

$$
\begin{aligned}
\int_{t+\Delta t}{ }^{t+\Delta t} \sigma_{i j} \delta_{t+\Delta t} e_{i j} d v & =\int_{t+\Delta t}{ }^{t+\Delta t} f_{i} \delta w_{i} d v \\
+\int_{{ }^{t+\Delta t} S^{f}}{ }^{t+\Delta t} t_{i} \delta w_{i} d s & ={ }^{t+\Delta t} R
\end{aligned}
$$

where the left superscripts refer to the configuration of the body and the left subscripts refer to the coordinate axes, and $e_{y}$ is the small strain tensor:

${ }_{t+\Delta t} e_{i j}=\frac{1}{2}\left({ }_{t+\Delta t} w_{i, j}+{ }_{t+\Delta t} w_{j, i}\right)$.

Note that:

$$
\delta_{t+\Delta t} e_{i j}=\delta \frac{1}{2}\left({ }_{t+\Delta t} w_{i, j}+{ }_{t+\Delta t} w_{j, i}\right){ }_{t+\Delta t} w_{i, j}=\frac{\partial w_{i}}{\partial^{t+\Delta t} x_{j}}
$$

\section{Incremental form}

The solution of Eq. (A7) requires the location of the body at time $t+\Delta t$. Therefore we transform Eq. (A7) to the configuration at time $t$, by defining 
the second Piola-Kirchoff stress ${ }^{t+\Delta t}{ }_{t} S_{i j}$ which refer to the stress at time $t+\Delta t$ but measured in the configuration at time $t$, as:

$\int_{{ }^{t} V}{ }^{t+\Delta t}{ }_{t} S_{i j} \delta^{t+\Delta t}{ }_{t} l_{i j} d v={ }^{t+\Delta t} R$

where

${ }^{t+\Delta t}{ }_{t} S_{i j}=\frac{{ }^{t} \rho}{{ }^{t+\Delta t} \rho}\left({ }_{t+\Delta t} x_{i, s}{ }^{t+\Delta t} \sigma_{s r t+\Delta t}^{t} x_{j, r}\right)$

${ }^{t+\Delta t} l_{i j}=\frac{1}{2}\left({ }_{t} w_{i, j}+{ }_{t} w_{j, i}+{ }_{t} w_{k, i t} w_{k, j}\right)$.

$l_{i f}$ is the Green-Lagrange strain tensor. Since the stresses and strains are unknown, the following incremental decompositions are used:

$$
\begin{aligned}
& { }^{t+\Delta t}{ }_{t} S_{i j}={ }^{t} \sigma_{i j}+{ }_{t} S_{i j} \\
& { }^{t+\Delta t}{ }_{t} l_{i j}={ }_{t} e_{i j}+{ }_{t} \eta_{i j} ;{ }_{t} e_{i j}=\frac{1}{2}\left({ }_{t} w_{i, j}+{ }_{t} w_{j, i}\right) ; \\
& { }_{t} \eta_{i j}=\frac{1}{2}{ }_{t} w_{k, i t} w_{k, j}
\end{aligned}
$$

Substituting Eq. (A12) into Eq. (A10) yields:

$$
\begin{aligned}
\int_{{ }^{t} V}{ }_{t} S_{i j} \delta_{t} l_{i j} d v & +\int_{{ }^{t} V}{ }^{t} \sigma_{i j} \delta_{t} \eta_{i j} d v={ }^{t+\Delta t} R \\
& -\int_{{ }^{t_{V}}{ }^{t} \sigma_{i j} \delta_{t} e_{i j} d v}
\end{aligned}
$$

When the stress increment ${ }_{t} S_{i f}$ is given in terms of incremental displacements, Eq. (A13) becomes a nonlinear equation in terms of incremental displacements. It should be noted that Eqs. (A7) and (A13) are, theoretically, equivalent.

When the rate of deformation is small, Eq. (A1) can be written as:

$$
{ }_{t} S_{i j}=D_{i j k l}\left(l_{t} e_{k l}-\Delta t \dot{\epsilon}_{k l}^{v p}\right)-\alpha \Delta t \dot{p} \delta_{i j}
$$

\section{Finite element formulation}

Eq. (A13) can be linearized as:

$$
\begin{gathered}
\int_{{ }^{t} V}{ }_{t} S_{i j} \delta_{t} e_{i j} d v+\int_{{ }^{t} V}{ }^{t} \sigma_{i j} \delta_{t} \eta_{i j} d v={ }^{t+\Delta t} R \\
-\int_{{ }^{t} V}{ }^{t} \sigma_{i j} \delta_{t} e_{i j} d v .
\end{gathered}
$$

Substituting Eq. (A14) into Eq. (A15) yields:

$$
\begin{aligned}
\int_{{ }^{t} V} D_{i j k l t} e_{i j} \delta_{t} e_{i j} d v & +\int_{{ }^{t} V}{ }^{t} \sigma_{i j} \delta_{t} \eta_{i j} d v={ }^{t+\Delta t} R \\
& -\int_{{ }^{t} V}{ }^{t} \sigma_{i j} \delta_{t} e_{i j} d v \\
& +\int_{{ }^{t} V}\left(\Delta t D_{i j k l} \dot{\epsilon}^{v p}\right. \\
& \left.+\alpha \Delta t \dot{p} \delta_{i j}\right) \delta_{t+\Delta t} e_{i j} d v
\end{aligned}
$$

When a standard formulation employing 8-node brick elements is used, approximating the displacement components in terms of its nodal values results in the following assembled matrix equation:

$$
\left(K_{L}+K_{N L}\right) \bar{w}={ }^{t+\Delta t} R-F-Z
$$

where

$$
\begin{aligned}
& K_{L}^{e}=\int_{{ }^{t} V^{e}} B_{L}^{T} D B_{L} d v \\
& K_{N L}^{e}=\int_{{ }^{V}{ }^{e}} B_{N L}^{T}{ }^{t} \sigma B_{N L} d v \\
& F^{e}=\int_{{ }^{t} V^{e}} B_{L}^{T} \hat{\sigma} d v \\
& Z^{e}=\int_{{ }^{t} V^{e}} B_{L}^{T}\left(\Delta t D \dot{\epsilon}^{v p}+\Delta t \alpha \dot{p}\right) d v \\
& K_{L}=\sum K_{L}^{e}, K_{N L}=\sum K_{N L}^{e}, F=\sum F^{e}, Z=\sum Z^{e}
\end{aligned}
$$

where the summation operator denotes the assemblage of the element matrices. In Eq. (A18) $B_{L}, B_{N L}, D, \hat{\sigma},{ }^{\mathrm{t}} \sigma$ are the linear strain-displacement transformation matrix, nonlinear strain-displacement matrix, elasticity coefficients matrix, Cauchy stress matrix, and vector of Cauchy stresses in the configuration at time $t$, respectively. It is important to realize that Eq. (A16) is an approximation to the actual equation to be solved, Eq. (A13). Depending on the nonlinearities in the system, the linearization of Eq. (A13) may introduce errors. For this reason it may be necessary to iterate at 
each time step until Eq. (A13) is satisfied to a required tolerance. The error is given by:

Error $={ }^{t+\Delta t} R-\int_{t+\Delta t}{ }^{t+\Delta t} \sigma^{l}{ }_{i j} \delta_{t+\Delta t} e^{l}{ }_{i j} d v$

where the superscript 1 refer to approximate values. We should note that the right-hand sides of Eqs. (A19) and (A16) are similar except the last integral arising from the visco-plastic strains.

\section{Iteration procedure}

Because of the inelastic effects, the solution is obtained in two steps. In the first step the nonlinear constitutive equations are solved for the incremental stresses. This requires the solution of six unknowns (components of stress tensor) at each selected point. We solve for the stress increments at the Gaussian points which results in an efficient integration of the integrals containing the stresses. If we use an implicit scheme, we can write:

${ }_{t} S_{i j}^{m}=D_{i j k l}\left({ }_{t} e_{k l}^{m-1}-\Delta t\left({ }^{(+\Delta t} \dot{\epsilon}_{k l}^{v p}\right)^{m}\right)+\Delta t \dot{p} \delta_{i j}$

where $m$ is the iteration number. The incremental displacement can be solved from:

$\left(K_{L}+K_{N L}\right) \bar{w}^{m}={ }^{t+\Delta t} R-F^{m}-Z^{m}$

We define the change in incremental displacement between iteration $m$ and $m-1$ as:

$\Delta \bar{w}^{m}=\bar{w}^{m}-\bar{w}^{m-1}$

Substituting Eq. (A22) into Eq. (A21) yields:

$\left(K_{L}+K_{N L}\right) \Delta \bar{w}^{m}={ }^{t+\Delta t} R-F^{m}-Z^{m}-\left(K_{L}+K_{N L}\right) \bar{w}^{m-1}$

Eq. (A23) can be rewritten by considering Eqs. (A13), (A17) and (A19) as:

$\left(K_{L}+K_{N L}\right) \Delta \bar{w}^{m}={ }^{t+\Delta t} R-{ }^{t+\Delta t} F^{m}$

where

${ }^{t+\Delta t} F^{m}=\int_{{ }^{t+\Delta t} V^{m}}{ }^{t+\Delta t} B_{L}^{m t+\Delta t} \hat{\sigma}^{m} d v$
The iteration continues until $\Delta w$ becomes reasonably small. When the solution at $t+\Delta t$ is obtained, the Cauchy stress in the deformed configuration is obtained using the relations:

$$
\begin{aligned}
& { }^{t+\Delta t} S_{i j}={ }^{t} \sigma_{i j}+{ }_{t} S_{i j} \\
& { }^{t+\Delta t} \sigma_{s r}=\frac{{ }^{t+\Delta t} \rho}{{ }^{t} \rho}{ }^{t+\Delta T}{ }_{t} x_{s, j}{ }^{t+\Delta t}{ }_{t} S_{i j}{ }^{t+\Delta t}{ }_{t} x_{r, j} .
\end{aligned}
$$

This completes the iteration for $t=t+\Delta t$. Since the solution is obtained in two steps, our numerical scheme can also be applied to problems with different kinds of constitutive relations. In such cases, one needs to modify the first step (i.e., solution of incremental stress components). The second step remains unchanged since it is a conservation equation.

Once the incremental displacements are solved, porosity can be solved from the mass balance of solids assuming incompressible solid grains:

$\frac{D \phi}{D t}=(1-\phi) \underline{D} \cdot \underline{u}$

It should be noted that since the viscosities and elastic parameters depend on the state of stress, failure function, and porosity, they are updated at every iteration.

To optimize the convergence rate, we have introduced a dynamic relaxation parameter for Eq. (A22). This parameter automatically changes depending on the convergence rate. An observation of Eq. (A24) shows that as the right-hand side of Eq. (A24) approaches zero, the incremental displacement vanishes, i.e., solution is obtained. This can be achieved regardless of a rigorous stiffness matrix, which is nothing but a preconditioner of the system. Therefore, we only calculate the linear part of stiffness matrix whenever appreciable changes in the system are observed and used it as a conditioner for the displacements.

\section{References}

Bathe, K.J., 1996. Finite Element Procedures. Prentice Hall, Englewood Cliffs, NJ. 
Bathe, K.J., Ramm, E., Wison, E.L., 1975. Finite element formulations for large deformation dynamic analysis. Int. J. Num. Meth. Eng. 9, 353-386.

Beard, D.C., Weyl, P.K., 1973. Influence of texture on porosity and permeability of unconsolidated sand. AAPG Bull. 57, 349-369.

Berkowitz, B., 1995. Analysis of fracture network connectivity using percolation theory. Math. Geol. 27, 467-483.

Berryman, J.G., 1980. Long-wavelength propagation in composite elastic media I: Spherical inclusions. J. Acoust. Soc. Am. 68, 1809-1819.

Berryman, J.G., 1986. Effective medium approximation for elastic constants of porous solids with microscopic heterogeneity. J. Appl. Phys. 69, 1136-1140.

Berryman, J.G., 1992. Single-scattering approximations for coefficients in Biot's equations of poroelasticity. J. Acoust. Soc. Am. 91, 551-571.

Biot, M.A., 1941. General theory of three-dimensional consolidation. J. Appl. Phys. 12, 155-164.

Biot, M.A., Willis, D.G., 1957. The elastic coefficients of the theory of consolidation. J. Appl. Mech. 24, 594-601.

Bour, O., Davy, P., 1998. On the connectivity of three-dimensional fault networks. Water Resource Res. 34, 2611-2622.

Chen, Y., Chen, W., Park, A., Ortoleva, P., 1994. In: Ortoleva, P. (Ed.), Role of pressure-sensitive reactions in seal formation and healing: Application of the CIRF A reaction-transport code. Basin compartments and seals AAPG Memoir No. 61. AAPG, Tulsa, Oklahoma, pp. 403-416.

Chen, M., Bai, M., Roegiers, J.-C., 1999. Permeability tensors of anisotropic fracture networks. Math. Geol. 31, 355-373.

Dewers, T., Ortoleva, P., 1990. Differentiated structures arising from mechano-chemical feedback in stressed rocks. EarthSci. Rev. 29, 283-298.

Dewers, T., Ortoleva, P., 1994. Nonlinear dynamical aspects of deep basin hydrology: fluid compartment formation and episodic fluid release. Am. J. Sci. 294, 713-755.

Druker-Prager, D.C., Prager, W., 1952. Soil mechanics and plastic analysis or limit design. Quart. Appl. Math. 10, 157-165.

Engelder, T., Geiser, P., 1980. On the use of regional joint sets as trajectories of paleostress fields during the development of the Appalachian Plateau New York. J. Geophys. Res. 85, 6319-6341.

Fischer, M.P., Gross, M.R., Engelder, T., Greenfield, R.J., 1995. Finite element analysis of the stress distribution around a pressurized crack in layered elastic medium-implications for the spacing of fluid-driven joints in bedded sediment-rock. Tectonophysics 247, 49-64.

Gassman, F., 1951. Uber die elastizität poröser medien. Veirteljahrsschrift der Naturforchenden Gesellschaft in Zürich 96, 1-23.

Ghaith, A., Chen, W., Ortoleva, P., 1990. Oscillatory methane release from shale source rock. Earth Sci. Rev. 29, 241-248.

Gross, M.R., 1993. The origin and spacing of cross joints examples from the Monterey formation, Santa-Barbara coastline, California. J. Struct. Geol. 15, 737-751.
Haase, C.S., Chadam, J., Feinn, D., Ortoleva, P., 1980. Oscillatory zoning in plagioclase feldspar. Science 209, 272-274.

Hancock, P.L., Ali Kadhi, A., Walper, J.L., 1984. Regional joint sets in the Arabian platform as indicators of interplate processes. Tectonophysics 3, 27-43.

Harris, J.F., Taylor, G.L., Walper, J.L., 1960. Relation of deformational fractures in sedimentary rocks to regional and local structures. AAPG Bull. 44, 1853-1873.

Hosterman, J.W., Dyni, J.R., 1972. Clay mineralogy of the Green River Formation, Piceance Creek Basin, Colorado A preliminary study. U.S. Geological Survey Professional 800, 159-163.

Kulander, B.R., Barton, C.C., Dean, S.L., 1979. The application of fractography to core and outcrop fracture investigations. U.S. Department of Energy, Morgantown Energy Technology Center Report METC/SP 79 (3), 174.

Larson, K.W., Waples, D.W., Fu, H., Kodama, K., 1993. Predicting tectonic fractures and fluid flow through fractures in basin modelling. In: Dore, A.G. (Ed.), Basin Modelling: Advances and Applications NPF Special Publications 3 Norwegian Petroleum Society. Elsevier, Amsterdam, pp. 373-383.

Lorenz, J.C., Teufel, L.W., Warpinski, N.R., 1991. Regional fractures I: A mechanism for the formation of regional fractures at depth in flat-lying reservoirs. AAPG Bull. 75, 1714-1737.

Luo, X., Vasseur, G., 1996. Geopressuring mechanism of organic matter cracking: numerical modeling. AAPG Bull. 80, 856-873.

Luo, X., Vasseur, G., Pouya, A., Lamoureux-Var, V., Poliakov, A., 1998. Elastoplastic deformation of porous medium applied to the modelling of compaction at basin scale. Mar. Petrol. Geol. 15, 145-162.

Mallory, W.W., 1977. Oil and gas from fractured shale reservoirs in Colorado and northwest New Mexico. Rocky Mountain Assoc. Geol. Spec. Publ. 1, 38.

Maubeuge, F., Lerche, I., 1993. A north Indonesian basin: geothermal and hydrocarbon generation histories. Mar. Petrol. Geol. 10, 231-245.

Maubeuge, F., Lerche, I., 1994. Geopressure evolution and hydrocarbon generation in a north Indonesian basin: twodimensional quantitative modelling. Mar. Petrol. Geol. 104, 104-115.

Maxwell, J.M., 1997. The physical chemistry and nonlinear dynamics of compartment formation in sedimentary basins. Ph.D. Thesis, Indiana University, Bloomington.

Multiwell Experiment Project Groups at Sandia National Laboratories and CER Corporation, 1987. Multiwell Experiment Final Report: I. The Marine Interval of the Mesaverde Formation: Sandia National Laboratories Report, SAND87-0327.

Multiwell Experiment Project Groups at Sandia National Laboratories and CER Corporation, 1988. Multiwell Experiment Final Report II. The Paludal Interval of the Mesaverde Formation: Sandia National Laboratories Report, SAND88-1008.

Multiwell Experiment Project Groups at Sandia National 
Laboratories and CER Corporation, 1988. Multiwell Experiment Final Report III. The Coastal Interval of the Mesaverde Formation: Sandia National Laboratories Report, SAND88-3284.

Multiwell Experiment Project Groups at Sandia National Laboratories and CER Corporation, 1988. Multiwell Experiment Final Report IV. The Fluvial Interval of the Mesaverde Formation: Sandia National Laboratories Report, SAND89-2612.

Nickelsen, R.P., Hough, V.N.D., 1967. Jointing in the Appalachian Plateau of Pennsylvania. Geol. Soc. Am. Bull. 78, 609-630.

Nicolis, G., Prigogine, I., 1977. Self-organization in non-equilibrium systems from dissipative structures to order through fluctuations. Wiley-Interscience, New York.

Nicolis, C., Nicolis, G., 1987. Irreversible phenomena and dynamical systems. Analysis in geosciences. D. Reidel Publishing Co., Dordrecht Holland.

Oda, M., 1985. Permeability tensor for discontinuous rock masses. Geotechnique 35, 483-495.

Oda, M., 1986. An equivalent continuum model for coupled stress and fluid flow analysis in jointed rock masses. Water Resources Res. 22, 1845-1856.

Oda, M., 1982. Fabric tensor for discontinuous geological materials. Soils and Foundations 22, 96-108.

Odling, N.E., 1992. Network properties of a two-dimensional natural fracture pattern. Pure Appl. Geophys. 138, 95-114.

Ortoleva, P., Hallet, B., McBirney, A., Meshri, I., Reeder, R., Williams, P., 1990. Self-organization in geological systems: Proceedings of a Workshop held 26-30 June. University of California at Santa Barbara Earth Sci. Rev. 29, 1-3.

Ortoleva, P., 1979. The multifaceted family of the nonlinear waves and fields center dynamics, catastrophes rock bands and precipitation patterns. In: Pacault, A., Vidal, C. (Eds.), Synergetics Far From Equilibrium. Springer-Verlag, New York, pp. 114-127.

Ortoleva, P., 1990. Self-organisation in Geological Systems Special Issue, Ortoleva, P. (Ed.), Earth Sci. Rev. Spec. Publ. 29.

Ortoleva, P., 1993. Self-organization and nonlinear dynamics in sedimentary basins. Phil. Trans. Roy. Soc. Lond. 344, 171-179.

Ortoleva, P., 1994a. Geochemical Self-organization. Oxford University Press, New York.

Ortoleva, P. (Ed.), 1994b. Basin Compartments and Seals, AAPG Memoir No. 61, AAPG, Tulsa, Oklahoma.

Ortoleva, P., 1994c. Development of diagenetic differentiated structure through reaction-transport feedback. In: Wolf, K.H., Chilingarian, G.V. (Eds.), in: Diagenesis 4, 39-52.

Ortoleva, P., Merino, E., Chadam, J., Moore, C.H., 1987a. Geochemical self-organization I: Reaction-transport feedback mechanisms and modeling approach. Am. J. Sci. 287, 979-1007.

Ortoleva, P., Merino, E., Moore, C.H., Chadam, J., 1987 b. Geochemical self-organization II: The reactive-infiltration instability. Am. J. Sci. 287, 1008-1040.
Ortoleva, P., 1998. Basin compartments and seals. Report prepared for the Gas Research Institute, No. GRI-97/0097.

Ozkan, G., Ortoleva, P., 1999a. Mechanical failure of cavities in poroelastic media. Submitted for publication.

Ozkan, G., Ortoleva, P., 1999b. Evolution of gouge grain size distribution: a Markov model. Submitted for publication.

Ozkan, G., Tuncay, K., Ortoleva, P., 1998. Process-based fault seal/conduit prediction,. 1998 AAPG Annual Convention Abstracts (CD-ROM format) May 17-28. AAPG, Salt Lake City, UT.

Payne, D., 1998. A new, fully coupled, reaction-transport-mechanical approach to modeling the evolution of methane reservoirs in the Piceance Basin. Ph.D. diss., Indiana University, Bloomington, IN.

Payne, D., Tuncay, K., Park, A., Comer, J., Ortoleva, P., 2000. Role of gas generation in natural fracture development and compartmentation in the Piceance Basin. AAPG Bull. (in press).

Renard, F., Ortoleva, P., Gratier, J.P., 1997. Pressure solution in sandstone: influence of clays and dependence on temperature and stress. Tectonophysics 280, 257-266.

Renard, F., Park, A., Ortoleva, P., Gratier, J.P., 1999. A transitional pressure solution model. Tectonophysics. (in press).

Rice, J.R., 1975. Continuum mechanics and thermodynamics of plasticity in relation to microscale deformation mechanisms. In: Argon, A.S. (Ed.), Constitutive Equations in Plasticity. MIT Press, Cambridge, MA, pp. 23-79.

Roberts, S.J., Nunn, J.A., 1995. Episodic fluid expulsion from geopressured sediments. Mar. Petrol. Geol. 12, 195-204.

Schneider, F., Potdevin, J.L., Wolf, S., Faille, I., 1996. Mechanical and chemical compaction model for sedimentary basin simulators. Tectonophysics 263, 307-317.

Segall, P., Pollard, D.D., 1983. Joint formation in granitic rock of the Sierra Nevada. Geol. Soc. Am. 94, 563-575.

Shaw, D.B., Weaver, C.E., 1965. The mineralogical composition of shales. J. Sed. Petrol. 35, 213-222.

Sonnenthal, E., Ortoleva, P., 1994.

Synder, M.D., Bathe, K.J., 1981. A solution procedure for thermo-elastic-plastic and creep problems. Nucl. Eng. Design 64, 49-80.

Thomson, M.E., Brown, S.R., 1991. The effect of anisotropic surface roughness on flow and transport in fractures. J. Geophys. Res. 96, 21923-21932.

Tuncay, K., Park, A., Ozkan, G., Zhan, X., Hoak, T., Sundberg, K., Ortoleva, P., 1998. Predicting the natural state of fractured carbonate reservoirs: Andector Field West Texas test of a 3-D RTM simulator. Paper in DOE/SAIC Final Report prepared for Contract DE-AC22-94PC91008.

Tuncay, K., Park, A., Ortoleva, P., 2000. A forward model of three dimensional fracture orientation and characteristics. J. Geophys. Res. (in press).

Turcote, D.L., 1992. Fractals, chaos, self-organized criticality and tectonics. Terra Nova 4, 4-12.

Ungerer, P., Burrus, J., Doligez, B., Chenet, P.Y., Bessis, F., 1990. Basin evaluation by integrated two-dimensional modeling of heat transfer, fluid flow, hydrocarbon generation and migration. AAPG Bull. 74, 309-335. 
Waite, M.E., Ge, S., Spetzler, H., Bahr, D.B., 1998. The effect of surface geometry on fracture permeability: A case study using a sinusoidal fracture. Geophys. Res. Lett. 25, 813-816.

Wang, C., Xie, X., 1998. Hydrofracturing and episodic fluid flow in shale-rich basins - a numerical study. AAPG Bull. 82, 1857-1869.
Wu, H.Q., Pollard, D.D., 1995. An experimental study of the relationship between joint spacing and layer thickness. J. Struct. Geol. 17, 887-905.

Zienkiewicz, O.C., Cormeau, I.C., 1974. Visco-plasticity and creep in elastic solids - a unified numerical solution approach. Int. J. Num. Meth. Eng. 8, 821-845. 


\section{Appendix C}

Tuncay, K., and P. Ortoleva. 2004. Quantitative basin modeling: present state and developments toward predictability, Geofluids, Vol. 4, 23-39. 


\title{
Quantitative basin modeling: present state and future developments towards predictability
}

\author{
K. TUNCAY AND P. ORTOLEVA \\ Laboratory for Computational Geodynamics, Department of Chemistry, Indiana University, Bloomington, IN 47405, USA
}

\begin{abstract}
A critique review of the state of quantitative basin modeling is presented. Over the last 15 years, a number of models are proposed to advance our understanding of basin evolution. However, as of present, most basin models are two dimensional (2-D) and subject to significant simplifications such as depth- or effective stress-dependent porosity, no stress calculations, isotropic fracture permeability, etc. In this paper, promising areas for future development are identified. The use of extensive data sets to calibrate basin models requires a comprehensive reaction, transport, mechanical (RTM) model in order to generate the synthetic response. An automated approach to integrate comprehensive basin modeling and seismic, well-log and other type of data is suggested. The approach takes advantage of comprehensive RTM basin modeling to complete an algorithm based on information theory that places basin modeling on a rigorous foundation. Incompleteness in a model can self-consistently be compensated for by an increase in the amount of observed data used. The method can be used to calibrate the transport, mechanical, or other laws underlying the model. As the procedure is fully automated, the predictions can be continuously updated as new observed data become available. Finally, the procedure makes it possible to augment the model itself as new processes are added in a way that is dictated by the available data. In summary, the automated data/model integration places basin simulation in a novel context of informatics that allows for data to be used to minimize and assess risk in the prediction of reservoir location and characteristics.
\end{abstract}

Key words: basin modeling, fluid flow, rheology

Received 16 September 2002; accepted 25 April 2003

Corresponding author: K. Tuncay, Laboratory for Computational Geodynamics, Department of Chemistry, Indiana University, Bloomington, IN 47405, USA.

E-mail: ktuncay@indiana.edu. Tel: +1812 8552717. Fax: +18128558300.

Geofluids (2004) 4, 23-39

\section{THE NEW BASIN MODELING}

Basin modeling, like any attempt to obtain quantitative predictions of a complex system, must be sufficiently comprehensive and, because of the great uncertainties involved, should be formulated in terms of probability theory. Here, we attempt to delineate steps that can be taken to achieve this goal and identify the benefits and pitfalls involved.

For the petroleum industry, a basin model is a set of rules (guided by laws underlying a set of physical and chemical processes) and computational techniques for predicting the location and characteristics of reservoirs. Thus, a model of a basin from this perspective is not just a sedimentologic history recreation software. It is not a maturation computation or a multiphase flow solver. It is not a rock deformation or heat transfer model. It is all this and more. And, in reality, it is also a methodology, either built into a software package or carried out by hand, to integrate all these physical and chemical processes with available geologic data.

Figure 1 suggests a set of reaction, transport, mechanical (RTM) processes that operate in a sedimentary basin. These processes are so strongly coupled that to leave out any of them is to risk failing to obtain reliable predictions of reservoir location and characteristics. For example, fracturing affects fluid flow, which changes pore fluid pressure and thereby fracturing, completing one of the many feedback loops underlying basin dynamics. Thus, while basin modeling apparently means many things to many people, we suggest that predictive basin models should be of the fully coupled, comprehensive RTM type.

The advantage of comprehensive RTM basin modeling is not only its potential for reliable predictions, but also its ability to predict a fuller suite of the parameters needed to characterize and evaluate a reservoir. They include the pressure 


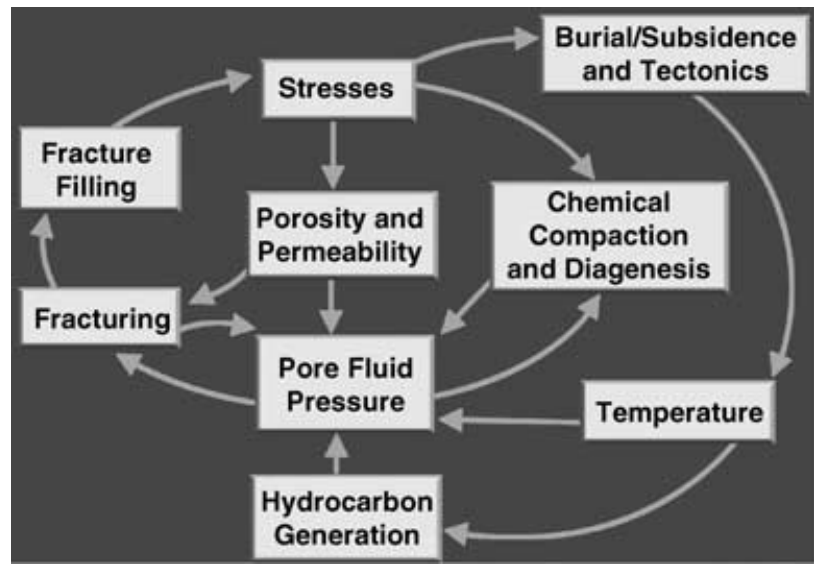

Fig. 1. Complex network of coupled processes underlying the dynamics of a sedimentary basin.

and composition of the various pore fluid phases, the shape, size, packing, and abundance of the minerals, fracture network statistics, and in situ stress. These rock and fluid parameters allow one to estimate reserves in place and the flow and mechanical properties of a reservoir needed to assess and optimize producibility.
We review a set of basin models and assessment of the subset of processes they account for in Table 1 . As the models are often under active development, and as the actual processes being accounted for are not always made explicit in the literature, Table 1 is only meant to be suggestive of the state of the art, rather than to be complete.

The complexity of basin models is not the only challenge we must face. The volume of basin data, obtained at great expense, presents both a technical and economic challenge to the industry. What is needed is an automated procedure to derive value from these databases often available on a field or basin. From our research, we suggest that automated analysis can be achieved through model-automated informatics (MAI). By definition, informatics is the science of deriving conclusions or otherwise deriving information from a vast and complex database. In this article, we suggest that this can be achieved with the use of comprehensive basin model to analyze available data using information theory. Before presenting the details of the MAI strategy, we summarize the present state of basin modeling and the improvements that will be needed to integrate basin modeling with information theory to make it an economically viable exploration and field development technology.

Table 1 An incomplete but representative list of basin models.

\begin{tabular}{|c|c|}
\hline Studies & Basin models \\
\hline Ungerer et al. (1990) & $2-\mathrm{D}, \phi E, H, \| \pi, \mathrm{SF}, \mathrm{PG}$ \\
\hline Forbes et al. (1992) & $2-\mathrm{D}, \phi E, H, \| \pi, \mathrm{PG}$ \\
\hline Person \& Garven (1992) & 2-D, $\phi E, H, \mathrm{I} \pi, \mathrm{PG}$ \\
\hline Maubeuge \& Lerche (1993) & $1-\mathrm{D}, \phi E, H, \mathrm{I} \pi, \mathrm{PG}$ \\
\hline Maubeuge \& Lerche (1994) & $2-\mathrm{D}, \phi E, H, \mathrm{I} \pi, \mathrm{PG}$ \\
\hline Bour \& Lerche (1994) & $2-\mathrm{D}, \phi E, H, \mathrm{I} \pi$ \\
\hline Bredehoeft et al. (1994) & $2-\mathrm{D}, \phi D, \mathrm{I} \pi, \mathrm{PG}, \mathrm{SF}$ \\
\hline Wieck et al. (1995) & $2-\mathrm{D}, \phi E, H, \mathrm{I} \pi$ \\
\hline Person et al. (1995) & $1-D, \phi D, H, \mid \pi, P G$ \\
\hline Luo \& Vasseur (1995) & 2-D, $\phi E, H, I$ \\
\hline Yu \& Lerche (1995) & $2-\mathrm{D}, \phi E, \| \pi, \mathrm{PG}$ \\
\hline Roberts \& Nunn (1995) & $1-\mathrm{D}, \phi E, H, \mathrm{I} \pi, \mathrm{SF}$ \\
\hline Burrus et al. (1996) & $2-\mathrm{D}, \phi D, H, \| \pi, \mathrm{PG}$ \\
\hline Luo \& Vasseur (1996) & $2-\mathrm{D}, \phi E, \| \pi, \mathrm{PG}$ \\
\hline Schneider et al. (1996) & 1-D, IS with elasticity and viscosity, $\mid \pi$ \\
\hline Mello \& Karner (1996) & $1-\mathrm{D}, \phi E, H, \mathrm{I} \pi, \mathrm{SF}$ \\
\hline Luo et al. (1998) & $2-D$, elastoplastic rheology (cam-clay model), $\mid$ \\
\hline Gordon \& Flemings (1998) & $1-\mathrm{D}, \phi E, \mathrm{I} \pi$ \\
\hline Wang \& Xie (1998) & 1-D, $\phi E, H, I \pi, S F$ \\
\hline McPherson \& Garven (1999) & 2-D, $\phi E /$ poroelasticity, $H, \mathrm{I} \pi$ \\
\hline Schegg et al. (1999) & $2-\mathrm{D}, \phi E, H, \| \pi, \mathrm{PG}, \mathrm{SF}$ \\
\hline Lee \& Williams (2000) & $2-\mathrm{D}, \phi E, H, \mathrm{I} \pi, \mathrm{PG}$ \\
\hline Suetnova \& Vasseur (2000) & 1-D, IS with elasticity and viscosity \\
\hline Payne et al. (2000) & 1-D, IS with elasticity, viscosity, and fracturing, $\| I \pi, S N D, P G$ \\
\hline Tuncay et al. $(2000 a, b)$ & 3-D, IS with elasticity, viscosity, and fracturing, II $\pi$, 3-D SND, PG \\
\hline Tuncay \& Ortoleva (2001) & 2-D, IS with elasticity, viscosity, and fracturing, $\| 1 \pi, 3-D$ SND, PG \\
\hline McPherson \& Bredehoeft (2001) & $3-D, \phi E, H, \| \pi, P G$ \\
\hline Stover et al. (2001) & $2-\mathrm{D}, \phi E, \| \pi, H, \mathrm{PG}$ \\
\hline
\end{tabular}

A list of earlier basin models was presented by Person et al. (1996). Representative list of basin models. Symbols indicate the following: $H$, heat flow; $1 \pi$, single phase flow; II $\pi$, two-phase flow; $\phi D$, porosity-depth relationship; $\phi E$, porosity-effective stress relationship; SF , simple fracture model; PG, petroleum generation; IS, incremental stress rheology; and SND, statistical fracture network dynamics. 


\section{THE NEED FOR COMPREHENSIVE 3-D RTM BASIN MODELING}

\section{Overview}

Nonlinearity and coupling are other reasons for constructing a comprehensive basin model. The modern theory of nonlinear dynamical systems has revealed their great potential for supporting a host of phenomena that arise autonomously, i.e. without their imposition by an external template. Nonlinear systems can, for example, oscillate periodically or chaotically in time and may organize spatially in regular patterns (see Nicolis \& Nicolis 1987; Ortoleva et al. 1987a,b, 1990; Turcotte 1992; Ortoleva 1994a).

A necessary condition for autonomous spatio-temporal organization is met when the system is maintained sufficiently far from equilibrium (Nicolis \& Prigogine 1977). The potential importance of nonlinear dynamics in geologic systems has been the subject of several conferences (Nicolis \& Nicolis 1987; Ortoleva et al. 1990) and has been investigated extensively in the context of geochemistry (Ortoleva 1994a). For the sedimentary basin, it has been pointed out (Ortoleva 1994a,b; Tuncay et al. 2000a) that nonlinear dynamics can play an important role at a wide range of spatial scales. This potentially autonomous behavior suggests that many patterns of mineralization, petroleum reservoirs, fault motion, and other phenomena cannot be understood as direct consequences of related patterns of sedimentology, volcanism, or tectonism, i.e. cannot be attributed to an external template. Because of the large network of processes underlying basin dynamics, as well as the nonlinearity of the conservation equations, a computational modeling approach is likely the only way to delineate nonlinear basin phenomena and the range of conditions (overall tectonics, sedimentation history, etc.).

Far-from-equilibrium conditions, necessary for the operation of autonomous spatio-temporal patterning, can be obtained in a sedimentary basin. The basin is sustained out of equilibrium by the fluxes and forces applied at its boundary. Input of sediment presents the basin with minerals and fluid chemical species, which, after burial, are out of equilibrium at the local pressure and temperature or other conditions. Changes in tectonic forces, heat flux, or influxed magmatic or meteoric fluids can also cause all or part of the basin to be driven out of equilibrium. Other factors are the effect of overburden on compaction and buoyancy-driven flow (oil, gas, or hot aqueous liquid). These factors that drive the basin out of equilibrium are directly or indirectly imposed at the basin boundaries. Thus, as suggested in Fig. 2, the conditions inducing change within a basin may be expressed in terms of the boundary conditions imposed on the solution of the conservation equations. Rapid burial, large geothermal gradients, and large amounts of chemically unstable kerogen are examples of factors favoring an increase in the likelihood

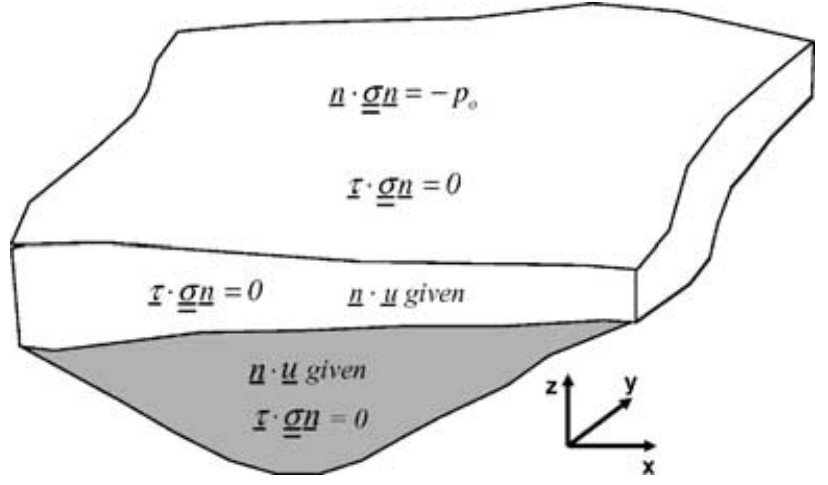

Fig. 2. Boundary conditions for stress $(\underline{\underline{\sigma}})$ shown here as well as those for heat and mass flow influence basin model predictions but are subject to great uncertainty. Conditions at boundary of basin simulation domain allow for imposition of ocean bottom normal pressure $P_{\mathrm{o}}$ and no shear at the top $(\tau$ being a unit tangent vector and $n$, an outward pointing unit normal vector with respect to the basin boundary). At the bottom, the tectonic history fixes the evolution of the deformation velocity $\underline{u}$ using slip conditions as on the sides (lightly shaded). The normal velocity $\underline{n} \cdot \underline{u}$ is imposed by the prescribed history of upheaval/subsidence and compression/extension.

of autonomous basin behavior. Comprehensive, threedimensional (3-D), fully coupled models are designed to capture this richness in autonomous basin behavior.

Although 1-D and 2-D studies give insights into the dynamics of basin evolution, a 3-D basin simulator is necessary to take all geometric effects into account. This becomes extremely important when fracturing is mainly because of flexure and the direction of tectonic compression/extension is changing over the basin's history. Fracture networks provide a pathway for fluid flow and, especially in a layered medium, fluids can move laterally. This can only be taken into account by a 3-D basin simulator with a stress/deformation solver that can capture the nonplanar layers (notably domes) and the strong variations in rheology from layer to layer. The fundamentally 3-D nature of these systems is further enhanced as preferred fracture orientation induces an anisotropic permeability tensor that can strongly influence the direction of fluid flow. Table 1 summarizes the spatial dimensionality and RTM processes of a number of basin models. In the next subsections, we discuss two important basin phenomena to illustrate the need for a comprehensive 3-D modeling approach.

\section{Compartmentation}

A compartment is a zone of fluid trapped within an envelope of low permeability rock. Compartmentation is viewed as a nonlinear phenomenon arising from the dynamics of the crustal fluid-rock system (Ortoleva 1994a,b, 1998; Ortoleva et al. 1995). This view leads to insights into the origins of compartments and the complex dynamics of the exchange of fluids with their surroundings. 
Compartments are found in sedimentary basins worldwide. Such domains of rock, typically with abnormal fluid pressures, have been recognized for many years in the petroleum industry (Dickey \& Cox 1977; Powley 1990; Al-Shaieb et al. 1994; Bradley \& Powley 1994; Ortoleva 1994a,b, 1998; Ortoleva et al. 1995). Powley (1980) has given evidence that sedimentary basins are typically divided into a boxwork of compartments, each of which are bounded on top, bottom, and sides by seals. Diagenetic, hydrologic, and mechanical processes underlying the development and dynamics of compartments are outlined by Ortoleva (1994a,b, 1998) and Ortoleva et al. (1995).

A key element of compartmentation appears to be the existence and inter-relationships between phenomena on a broad range of length scales. What is, in fact, most interesting and important is that the phenomena on these wide range of scales may strongly couple to reinforce each other in many ways. Let us define these scales, and then note some of their inter-relationships.

Microscale phenomena are defined here to be those that take place on a length scale of the order of a single or perhaps few grain diameters. Most notable among these phenomena are grain growth/dissolution/nucleation reactions, grain coating, plastic grain deformation, microfracturing/breakage, and rearrangement. These processes underlie compaction and porosity/permeability occlusion or enhancement.

Mesoscopic phenomena take place on the 10-grain diameter to meter scale. A key aspect of mesoscopic phenomena is that they involve a statistically significant number of grains so that they might be described in terms of the spatial distribution of the local texture. Texture is taken to mean the average grain volume, shape and orientation of the various minerals present but could be more detailed - that is, be described in terms of the probability distributions for the aforementioned quantities. Examples of mesoscopic phenomena are stylolites, banded compaction/cementation alternations, differentiated or enhanced marl/limestone alternations, and banded carbonate cements in sandstones (Dewers \& Ortoleva 1990a, 1994a; Ortoleva 1994a,b; Qin \& Ortoleva 1994).

Macroscopic phenomena are exemplified by intra-lithologic unit compartments or those encompassing several lithologic units. They exist on the $10-\mathrm{m}$ to $10-\mathrm{km}$ scale, laterally. The largest (basin) scale is the megascopic scale. A megacompartment may have a complex, nested interior structure of smaller scale compartments.

Phenomena on all these scales are intimately related. Mesoscopic processes involve the spatial distribution of microscopic variables. In turn, the conditions promoting or repressing the processes (stress or fluid pressure, for example) are affected by macro- and megascopic phenomena. Mesoscopic phenomena can create seals and hence play a key role in defining the boundaries of a compartment on the macroor megascopic scale. In summary, phenomena on one scale affect and are affected by phenomena on one or more other scales - compartmentation involves a complex network of cross-coupled processes operating on a hierarchy of scales.

\section{Salt tectonics}

While salt tectonic phenomena have long been recognized as a key process in basin evolution and, in particular, in the context of petroleum reservoir location and characteristics, most studies have not addressed the complexities caused by the interplay of salt deformation with other RTM processes.

Salt tectonics features are the consequences of a symmetry breaking of the idealized planar state of a salt layer over- and under-lain by other horizontal sediments. Small disturbances in the translational invariance of the system in the horizontal directions can be amplified by the RTM processes into wave, diapir, and more complex features. The controversy as to the physical laws underlying salt tectonics, and hence, in our view, the aforementioned self-organization, still has not been completely resolved (Koen 1993; Taylor 1995; Tuncay \& Ortoleva 2001). We believe that this is because, while one can rather easily conjecture a variety of mechanisms, it is likely a quantitative question as to which ones dominate in a given geologic context. Experience gained over the past three decades in nonlinear dynamical systems shows that they often behave in rather unexpected ways. A solution to this dilemma is to study salt tectonics using a comprehensive, 3-D multiprocess simulator. In such an approach, one may test the relative importance of the various factors and evaluate the degree to which they reinforce or inhibit one another.

Although there is a vast number of observations of salt structures (Seni \& Jackson 1984; Jackson \& Talbot 1986; Cobbold 1993), there are only a few attempts to numerically simulate them. Most salt simulators are 2-D, and both salt and sediment are treated as bulk materials, i.e. fluid flow through the pores and its influence on effective stress are ignored. None of these models account for the evolving rheology of the sediments because of diagenesis and mechanical processes, petroleum generation, and the changing thermal regime. Therefore, most salt tectonics simulators do not have the sufficient comprehensiveness that can assist saltrelated exploration.

Daudre \& Cloetingh (1994) presented a 2-D analysis based on Stokes flow. In this approach, both sediment and salt are treated as nonlinear viscous fluids. A Drucker-Prager criterion is adopted to model the inception of brittle deformation. The density of sediment was calculated based on a pressure-dependent porosity. An updated Lagrangian formulation was used to simulate large deformations. They showed that extension combined with salt rheology favors salt diapirism. Van Keken et al. (1993) obtained the effective rock salt viscosity by adding dislocation creep and pressure solution creep. The effective salt viscosity was allowed to depend on temperature, strain rate, and grain size. Pressure solution dominated the salt motion. Sediment density was taken to 
be a function of depth. With these assumptions, they performed a sensitivity analysis for the effect of overlying sediment viscosity. Their results showed that the sediment viscosity/salt viscosity ratio is an important factor in determining salt motion and geometry. Another 2-D model was developed by Mazariegos et al. (1996) based on Stokes flow. They used two different salt rheologies; a dislocation-creep power law and a fluid-assisted creep law. The functional form of the salt rheology depended on grain size. Although they used a rather complex rheology for salt, sediment viscosity was taken to be constant. They showed that their fluidassisted creep law results in faster salt motion. They concluded that a better understanding of salt diapirism requires an accurate characterization of surrounding rock rheology. A 2-D study was presented by Schultz-Ela et al. (1994) who used the commercial program GEOSIM-2D for their numerical modeling. Salt was treated as a visco-elastic material, whereas sediment was modeled with an elastoplastic rheology. Plastic yield was determined with a Drucker-Prager criterion.

In all of these studies, the results were limited to 2-D. However, the most crucial difficulty is that they are limited to the deformation of bulk media. In other words, they do not employ the theory of composite porous media. In composite environments, the velocity, stress, and other descriptive variables of solid and fluid phases show very different time history and spatial distributions. For example, the velocity and stress tensor of solid and fluid phases are very distinct. Therefore, although approximation of such a complicated composite medium by an equivalent bulk medium may give some information, it cannot have the predictive power of a composite model.

Recently, Tuncay \& Ortoleva (2001) presented a 3-D model that accounts for poroelasticity, nonlinear temperature-dependent viscosity, 3-D fracture network dynamics, and multiphase flow with petroleum generation. They also studied the role of the coupling between the spatial distribution of sediment input rate and diapir growth, and demonstrated the genesis of subsalt compartments, coordinated migration of petroleum and fracture network dynamics, and salt morphology.

\section{IMPORTANT PHYSICAL AND CHEMICAL PROCESSES}

\section{Stress and deformation}

Reconstructing the stress and deformation history of a sedimentary basin is a challenging and important problem in the geosciences with a variety of applications. These include petroleum exploration, reserve assessment and production, and earthquake hazard reduction. Progress in this field has been hampered by the absence of an integrated mechanical modeling approach set within the wider context of the coupled RTM dynamics of a basin.
The strongly coupled nature of basin deformation may be understood in terms of the feedbacks underlying crustal dynamics. For example, pore fluid pressure affects stress, stress changes can lead to fracturing, and fracturing can affect pore fluid pressure. Similarly, stress can affect mineral solubility, the latter can cause mineral dissolution which, in turn, can affect rock rheology and hence stress. Clearly, basin deformation requires an accounting of the coupling among the many RTM processes. Therefore, modeling sedimentary basin dynamics requires a fully coupled approach.

The goal of the modeling is to calculate the evolution of the distribution within a basin of the set of descriptive variables characterizing its internal state:

- rock texture and mineralogy;

- fluid properties;

- temperature;

- rock deformation;

- fracture network characteristics; and

- stress.

These properties respond over geologic time to their interactions among each other and via the influence of the basin's surroundings. The interaction with the surroundings provides the boundary conditions to which the equations of mass, energy, and momentum conservation must be subjected to arrive at the evolution of the basin. In this way, basin analysis becomes the delineation of the RTM basin dynamical system and its response to the constraints imposed at the boundaries. As the laws for the basin RTM processes are nonlinear in the descriptive variables, one expects this response to be extremely rich (Dewers \& Ortoleva 1994a; Ortoleva 1994a,b, 1998).

An important limiting assumption made in most basin models is that there exists a simple dependence of porosity on effective stress (Ungerer et al. 1990; Forbes et al. 1992; Person \& Garven 1992; Maubeuge \& Lerche 1993, 1994; Bour \& Lerche 1994; Luo \& Vasseur 1995, 1996; Roberts \& Nunn 1995; Wieck et al. 1995; Yu \& Lerche 1995; Yu et al. 1995; Mello \& Karner 1996; Schneider et al. 1996; Gordon \& Flemings 1998; Wang \& Xie 1998; McPherson \& Garven 1999; Schegg et al. 1999; Lee \& Williams 2000; Polyansky \& Poort 2000; McPherson \& Bredehoeft 2001) or depth (Bredehoeft et al. 1994; Person et al. 1995; Burrus et al. 1996). Different compaction parameters are used for shales and sandstones regardless of their mineral composition, and grain size distribution. Although these approximations allow fast computations, they lack the effects of compressional/extensional regimes, brittle deformation (such as fracturing), heat flux, and rock texture on porosity evolution. Such expressions might result in limited success for a well-studied basin with a large set of data. However, their applicability to new prospects is doubtful as the parameters calibrated for a mature basin include the unique geologic boundary histories (overall tectonics, heat and fluid flux at the boundaries) that influenced it. In summary, these 
models do not account for the unique history of pressure, stress, and temperature to which the medium was subjected. The conservation laws are universal; it is the differences in the history of the evolving boundary conditions that give a basin its individual character.

The difficulty in modeling deformation in porous media stems from the many deformation processes that operate over long time scales. The integration of these processes can be achieved using an incremental stress approach. In analogy with the classic theory of chemical kinetics, the total rate of strain $\underline{\underline{\varepsilon}}$ is written as a sum of terms

$\dot{\underline{\varepsilon}}=\sum_{j=1}^{N} \underline{\underline{\dot{\varepsilon}}}^{(j)}$.

The list of such processes includes:

- poroelasticity;

- continuous, irreversible rock deformation;

- fracturing; and

- pressure solution.

The outstanding contributions of incremental stress theory is that the total rate of strain is expressible as a linear combination of rate of strains from different processes due to the fact that it represents a relation among infinitesimal changes. The individual rate of strain terms on the right-hand side of equation 1 depend on the full suite of rock textural and fluid properties as well as the macroscopic stress. it is through this dependence and the coevolution of rock deformation and of these variables that the full coupling of all processes is accounted for in a model. As the rates $\underline{\dot{\varepsilon}}^{(j)}$ typically vary more strongly than linearly with these variables, the basin is a nonlinear dynamical system.

The poroelasticity term depends on the elasticity tensor of the medium, effective stress coefficient, total stress, and fluid pressure. The elasticity tensor depends on the instantaneous mineral composition, fracture statistics, grain sizes, temperature, and porosity. As these quantities (fracture statistics, temperature, etc.) evolve, the elasticity parameters change accordingly. The challenge is the calculation of the individual rates of strain for a multimineralic rock as it requires the homogenized response of the medium. For example, given the mineral elasticities, grain sizes, and porosity, the bulk and shear moduli, and effective stress coefficients of the (assumed) isotropic rocks can be computed using Berryman's composite medium theory (Berryman 1980, 1986, 1992).

A direct coupling of mechanics and chemistry arises through pressure solution. Grain dissolution at stressed grain-grain contacts induces compaction and, thereby, contributes to the total rate of strain. The rate of this pressure solution contribution, depends on the stress at grain-grain contacts and hence on the macroscopic stress, fluid pressure, and texture. However, it should also depend on the composition of the pore fluid. Pressure solution-derived rates of strain must be formulated through a relation between the rate of change of variables characterizing the texture and through a geometric relation between texture and macroscopic strain (Weyl 1959; Dewers \& Ortoleva 1994b; Renard et al. 1999a,b).

Another effect that is usually disregarded is the volumetric strain caused by fracturing. As fractures open, the overall rock volume increases and fluid pressure decreases (because of flow and increase in pore volume). This reduces the rate of fracture growth. Therefore, fracturing is a self-limiting process.

Example boundary conditions that are needed to complete the formulation of the rheologic problem are illustrated in Fig. 2. These conditions enforce the lateral compression/ extension and subsidence/upheaval imposed by the larger scale tectonics. The interaction of the top of the sediment pile with the overlying fluids (atmosphere or sea bottom) is accounted for by the value of normal stress and the (assumed) absence of tangential shear. A no-vertical-shear lateral boundary condition allows for natural compaction at the sides of the basin.

There are a few basin-modeling studies that consider incremental stress rheology. Among them, one can note Dewers \& Ortoleva (1994a; 1-D incremental stress rheology accounting for poroelasticity and pressure solution with single-phase flow), Suetnova \& Vasseur (2000; 1-D visco-elastic rheology with single phase flow), Schneider et al. (1996; 1-D visco-elastic rheology with single phase flow), Luo et al. (1998; 2-D elastoplastic rock behavior with single phase flow), Tuncay et al. (2000a,b), and Tuncay \& Ortoleva (2001; 3-D incremental stress rheology accounting for poroelasticity, nonlinear viscosity, and fracturing with multiphase flow).

\section{Multiphase flow}

Multiphase flow in porous media is of interest in various areas of science. Significant amount of effort has been spent on different aspects and applications of multiphase flow in porous media such as contamination of groundwater aquifers, reservoir modeling, and basin evolution. The main difference between these applications is the magnitude of the time scale. The engineering time scale contamination or recovery operations can vary from days to months, whereas the time scale for basin evolution is in the order of millions of years. Therefore, the numerical approach followed for large time scale problems must allow large time steps, and must be accurate enough to capture the nonlinear dynamics of the problem. Furthermore, the dynamic nature of the basin evolution because of the time-dependent sedimentation, extension, subsidence, and erosion makes the problem computationally very challenging. Another complexity is the range of material properties such as permeability in basins. The permeability range may be as large as 10 orders of magnitude. This requires the use of a mesh that is locally adapted to the material discontinuties. 
Numerical models for multiphase flow in porous media have been presented by various researchers based on finite difference and finite element methods. These models focused on surface spills and subsurface leakage of hydrocarbons from pipes and storage tanks (Kaluarachchi \& Parker 1989; Celia \& Binning 1992; Sleep \& Sykes 1993a,b; Huyakorn et al. 1994; Panday et al. 1994, 1995; White 1995; Hadad et al. 1996) and reservoir simulation (Young \& Stephenson 1983; Watts 1986; Quandalle \& Sabathier 1989; Sukirman \& Lewis 1993). In parallel with the advances in computer hardware, the simulators have started to employ implicit numerical techniques with applications to miscible multiphase flow problems. Newton-Raphson linearization appears to be the most popular technique in the solution of nonlinear algebraic equations, which makes the need for fast largesparse-matrix solvers inevitable (Kipp et al. 1992; Peters 1992). A review of trends in numerical modeling of multiphase flow in porous media is given by Panday et al. (1995).

The potential role of petroleum in creating traps and preserving the porosity needed to house it has long been recognized in the petroleum industry (Wilson 1977; O'Brien \& Lerche 1986). Multiphase flow models in basin simulators have mostly been limited to immiscible two-phase flow (Ungerer et al. 1990; Forbes et al. 1992; Burrus et al. 1996; Luo \& Vasseur 1996; Tuncay \& Ortoleva 2001) or miscible two-phase flow (Payne et al. 2000; Tuncay et al. 2000a). Yu \& Lerche (1995), McPherson \& Bredehoeft (2001), Wendebourg (2000) and Schegg et al. (1999) presented three-phase multiphase flow in the context of basin modeling. Modeling of multiphase flow in deforming porous media requires rock texture- and fluid composition-dependent relative permeability and capillary pressure curves. In the absence of experimental data, expressions provided by Brooks \& Corey (1964) and Van Genuchten (1980) are the most commonly used ones (see Lenhard et al. 1989b for a comparison). Although empirical parameters are introduced to model the observed hysteresis in flow parameters (Parker \& Lenhard 1987; Lenhard et al. 1989a), they have not apparently been applied to basin scale problems yet.

\section{Petroleum generation}

The thermal degradation of organic material leads to the creation of components (methane and other C- and H-rich molecules), which add to the pore-filling fluid phases. For organicrich lithologies [coal or high total organic carbon (TOC) shale], organic reactions can cause compaction or shrinkage. Reaction-induced matrix shrinkage causes cleating in coals and may lead to similar effects in organic-rich shales. Models of kerogen breakdown and other organic reactions are required to study the time course of the generation of petroleum. A number of such models are available (Tissot et al. 1987; Espitalie et al. 1988; Braun \& Burnham 1990; Hunt et al. 1991; Pepper \& Corvi 1995). However, these models are highly schematic and are calibrated to the organic material from various basins but not to the detailed network of reaction processes among specifically identified molecules or sites on macromolecules. Thus, it seems that results from one type of material, basin, or context cannot be easily generalized to others.

A fundamental component of most existing basin models is petroleum generation (Ungerer et al. 1990; Forbes et al. 1992; Person \& Garven 1992; Bredehoeft et al. 1994; Person et al. 1995; Yu \& Lerche 1995; Burrus et al. 1996; Luo \& Vasseur 1996; Mello \& Karner 1996; Forster et al. 1998; Schegg et al. 1999; Lee \& Williams 2000; Payne et al. 2000; Wendebourg 2000; Tuncay et al. 2000a; McPherson \& Bredehoeft 2001; Tuncay \& Ortoleva 2001; Payne \& Ortoleva 2002a,b). The generated petroleum is used as a lumped source term in the mass balance equation for the oil or gas phase, i.e. the detailed composition of products is not taken into consideration.

To overcome the limitations of relatively simple petroleum generation models, the following must be addressed:

- More complex equations of state.

- Detailed mechanisms allow greater number of activation energies and thus greater predictability of the relations between thermal histories and gas composition.

- Temperature/overpressure relationship.

- Coupling to organic reactions.

\section{Heat flow}

It is a common practice to assume that the solid phase is in thermal equilibrium with the fluid phase. Classical advective-dispersive heat transfer equation is used to model heat flow (Ungerer et al. 1990; Forbes et al. 1992; Person \& Garven 1992; Person et al. 1995; Roberts \& Nunn 1995; Wieck et al. 1995; Mello \& Karner 1996; Forster et al. 1998; Wang \& Xie 1998; McPherson \& Garven 1999; Polyansky \& Poort 2000; McPherson \& Bredehoeft 2001). A specified temperature is imposed at the interface between sediment pile and sea, and heat flux history is applied at the bottom of the sediment pile. The lateral boundaries are assumed to have zero heat flux. Some models have considered radiogenic heat production (Mello \& Karner 1996; Forster et al. 1998).

As rheology is affected by temperature, and as deformation affects, the spatial distribution of thermal conductivity, heat transfer, and deformation problems must be solved simultaneously - an issue particularly important in salt tectonic regimes (Tuncay \& Ortoleva 2001).

\section{Fracture mechanics}

Fractures play an important role in many geologic processes. They provide a mechanism of deformation and a pathway for fluid flow. The timing of fracture initiation and the scenario of their evolution over the history of a zone may significantly affect the rate and direction of fluid migration. 
Because of its importance to both petroleum and geologic sciences, fracturing has been studied by many researchers (see Pollard \& Aydin 1988; Lorenz et al. 1991 for reviews). Fractures in areas subjected to bending are usually explained by associated extensional stresses (Friedman 1976). The existence of fractures in near-horizontal layers has been attributed to unloading (Currie \& Nwachukwu 1974; Engelder 1987), high fluid pressure (Pollard \& Aydin 1988; Ortoleva 1998), and anisotropic stress influenced by nearby geologic structures (Currie \& Nwachukwu 1974; Segall \& Pollard 1983). Clearly, fracturing in near-horizontal or folded areas is because of a combination of the effects listed above. If the local fracture kinetics is well described by fracture growth/ healing laws, a multiprocess deformation model coupled to fluid flow and fracturing can be used to quantify the relative importance of these effects.

It is well documented that fracturing strongly depends on lithology (Kulander et al. 1979; Segall \& Pollard 1983; Hancock et al. 1984; Lorenz et al. 1991; Gross 1993; Fischer et al. 1995; Wu \& Pollard 1995). Although fracturing can occur in almost any type of rock, they are more common in brittle rocks (Mallory 1977). Furthermore, fractures in a brittle lithology commonly discontinue at the interface of more ductile lithologies (Engelder \& Geiser 1980). Another observation is that fracture spacing is strongly dependent on bed thickness and lithology (Harris et al. 1960; Nickelsen \& Hough 1967; Gross 1993; Fischer et al. 1995; Wu \& Pollard 1995). However, a simple correlation between fracturing and bed thickness does not seem feasible because of the many factors operating such as fluid pressure, state of stress, neighboring lithology properties, and tectonics.

Present-day flexure is often a poor indicator of fracturing. If the rate of flexure development was very slow, then there would be no fracturing. Also, flexure early in a sedimentary rock's evolution (i.e. when it is poorly lithified) or if sediment has inherent ductile behavior (as for organic-rich shales, rock salt or anhydrites) can occur without fracturing. Thus, prediction of present-day fracturing requires a model that allows for the continuous processes (notably ductile behavior or pressure solution) to compete with discontinuous deformation (faulting and fracturing) to arrive at the overall deformation.

Fracturing may introduce anisotropy to the evolving system. Fractures orient flows along their surface and also introduce directions of weakness in a deforming rock. Thus, fractures may guide the direction of high pressure fluids, which thereby changes the location of subsequent fracturing. Fracture-induced changes in rheologic parameters may affect the overall stress tensor, which, in turn, affects the orientation of subsequent fracturing.

In most basin models, it is assumed that rocks fracture when the fluid pressure exceeds a specified fraction of lithostatic (Ungerer et al. 1990; Maubeuge \& Lerche 1993, 1994; Bredehoeft et al. 1994; Roberts \& Nunn 1995; Luo \& Vasseur 1996; Mello \& Karner 1996; Schneider et al.
1996; Wang \& Xie 1998). This assumption essentially eliminates the dependence of fracturing on lithologic properties, although it is well known that fracturing strongly depends on texture (Harris et al. 1960; Nickelsen \& Hough 1967; Mallory 1977; Segall \& Pollard 1983; Lorenz et al. 1991; Gross 1993; Fischer et al. 1995; Wu \& Pollard 1995). Such a simplification also fails in predicting nonvertical fractures because of flexure.

In most of the existing basin evolution simulators, fracture permeability is assumed to be isotropic (Ungerer et al. 1990; Maubeuge \& Lerche 1993, 1994; Bredehoeft et al. 1994; Roberts \& Nunn 1995; Luo \& Vasseur 1996; Mello \& Karner 1996; Schneider et al. 1996; Wang \& Xie 1998). This is apparently because of the lack of information in their models about the tensorial nature of the stress tensor and the resulting, evolving fracture network.

There is a vast amount of work on approximations for the permeability of fracture networks (for example, Oda 1986; Long \& Billaux 1987; Odling 1992; Berkowitz 1995; Koudina et al. 1998). However, in these studies, a fracture network is generated either by an independent (decoupled) statistical, geometrical model or based on data.

To account for the above effects, forward quantitative basin models should include:

- fracture orientation reflecting the stress tensor;

- new fractures are added to the evolving network as the stress tensor changes because of tectonics or to fracture or diagenetic changes in rheology;

- the fracture network characteristics affect the tensorial fluid transport (e.g. permeability) and rock rheologic parameters;

- the construction of rose diagrams and other parameters that can be compared with observation; and

- an account of the mineralogy, texture, and statistical variations of properties (notably weak spots) that can affect fracturing and change over the geologic time scale.

Recently, Tuncay et al. (2000b) presented a dynamical model of fracture growth that is fully coupled to other crustal processes, deformation, and heat transfer. Thus, the oscillatory and other self-organization and nonlinear phenomena of crustal evolution can be captured (Ortoleva et al. 1987a,b; Maxwell \& Ortoleva 1994; Dewers \& Ortoleva 1994a; Ortoleva 1994a,b). The most notable of these is the cycle of fracturing $\rightarrow$ fluid flow $\rightarrow$ fluid pressure release $\rightarrow$ fracture healing (Ghaith et al. 1990; Chen et al. 1994; Dewers \& Ortoleva 1994a; Ortoleva 1998). Modifications were made to the fracture length growth law that allows for fracture healing. In their model, each representative volume of rock is given a statistical distribution of fracture nuclei and associated tensile strength. This is a way to approximate the effect of rock heterogeneity and fracture interaction, and to predict the density of fractures and their dependence on the histories of stress, fluid pressure, and rock properties. A formalism is developed to account for the orientation of single or multiple fracture sets. The model is thereby able to describe 
conditions for developing a few large fractures or a swarm of smaller ones, depending on the rate of change of fluid pressure and stress. The change in the rock volume and anisotropic fracture permeability tensor are also obtained.

\section{UNCERTAINTIES IN BASIN MODELS}

\section{Overview}

Although basin models require a large number of phenomenologic parameters as well as geologic boundary conditions, only a few studies focused on the utilization of observed data to constrain the model (Lerche 1991; Maubeuge \& Lerche 1993; Zhao \& Lerche 1993; Yu et al. 1995; Tuncay \& Ortoleva 2002). Yu et al. (1995) used observed porosity, permeability, fluid pressure, and layer thickness data to evaluate two parameters that appear in empirical porosity and permeability expressions. However, their study lacks the assessment of uncertainty associated with the predictions.

Uncertainties in the input data needed to run a basin model lead to uncertainties in the predictions. Furthermore, formulating this input data is an extremely labor-intensive and subjective process. We suggest that basin modeling is naturally placed within the context of probability theory as follows. Let $\rho[B]$ be the probability of the boundary tectonic scenario $B$. The objective is to construct $\rho[B]$ and thereby find the most probable $B$. Once the most probable $B$ is determined, we can use it with the basin model to predict the likely location and characteristics of the reservoirs in a study area. As we have $\rho[B]$, we can also determine the uncertainties in any of the reservoir location and state parameters. With this, the basin-modeling effort should focus on the development of basin data collection procedures that reduce the uncertainties implied quantitatively by the form of the dependence of $\rho$ on $B$.

As the objective is to decrease uncertainty, we wish to obtain sufficient information to limit the range in $B$ over which $\rho$ is nonnegligible. Information theory (Jaynes 1957) provides a general prescription for constructing $\rho[B]$ using the information we know about the system. This procedure is outlined in Information theory formulation. It is shown there that seismic, well-log, core, and other data can be used to constrain $\rho$ if the basin model is sufficiently comprehensive that it predicts enough fluid and rock properties to construct synthetic seismic, well-log and other data. While the difference between the observed and synthetic data provides an error that can be minimized to calibrate a few model parameters, it cannot, given the sparseness of real data sets, give the most probable boundary tectonic scenario, nor can such an error minimization procedure yield a self-consistent assessment of risk. The procedure outlined in Information theory formulation enables one to surmount these difficulties.

A probabilistic basin-modeling approach can also provide a natural platform for the integration of expertise. These exper- tise constraints include limiting the spatial and temporal scale of phenomena (e.g. maximum known rate of overall basin deformation, basement heat flux, etc.). In the section under Information theory formulation, we show that this can conveniently be performed via the minimum relative entropy approach. As a model becomes more comprehensive or the number of expertise constraints are increased, less data are needed. If the above data/modeling integration can be automated, a basin model in effect becomes the centerpiece of a database mining algorithm as differential equations of physics and chemistry are simply algorithms for processing information. In this sense, this procedure is the essence of a quantitative geoinformatics methodology.

\section{Phenomenologic parameters}

Modeling a large number of RTM processes requires information on a rather large set of parameters that appear in the equations of mass, momentum, and energy conservation, and phenomenologic laws. Most of these parameters are subject to uncertainties.

\section{Rock rheology}

The bulk and shear elastic moduli of minerals are fairly well known. However, existing composite medium approximations are based on wave scattering for simple geometries. The effective viscosity of a multimineralic rock poses one of the most difficult homogenization problems. As experimental data in the range of temperatures of interest are very limited, parameters that appear in the phenomenologic expressions are subject to great uncertainty. Currently, most of the experimental studies in this area focus on the compaction. Even if the medium is isotropic, there is need for additional information on the irreversible distortional rock deformation.

\section{Multiphase flow parameters}

The relative permeability and capillary pressure relations evolve as rock texture and fluid composition change. Therefore, using present day relationships throughout the simulations is a very crude approximation. There is need for development of expressions that account for grain size distribution, porosity, fluid composition, and temperature. Calculation of matrix and fracture permeability tensors requires a sufficiently rich texture model that includes many variables to describe the medium. This aspect is briefly discussed in the section under Incorrect and/or incomplete models.

\section{Geologic boundary conditions}

For practical reasons, basin analysis is based on sparse and irregularly distributed data. Basin analysis involves reconstructing the spatial geometry of sedimentary bodies in three dimensions or reconstructing the detailed history of sediment 
input/erosion across the basin, and a number of commercial software focus on this aspect of basin modeling. Such information is invaluable in unraveling the history of the basin and for applications such as mineral and oil exploration, hydrocarbon reservoir simulation, and resource assessment.

To be effective, such a reconstruction must have a number of key attributes:

- automation so that new data can continuously be added for re-evaluation;

- automatic output of cross-sections and 3-D graphical representations;

- respect of general knowledge of sedimentary body geometry, sharpness of property changes across contacts, and smooth variations within a lithologic unit; and

- respect of irregularity of well location and depth/time interval of available mineralogic, age, and textural information.

Thus, an effective approach must transform sparse, irregularly distributed data into a basin-covering picture that respects notions of sedimentology and can be continuously and automatically updated.

Perhaps the most error-prone of basin model input data is the 'tectonic boundary scenario' $B$ :

$$
B=\text { time course of }\left\{\begin{array}{l}
\text { uplift/subsidence } \\
\text { compression/extension/wrenching } \\
\text { basement heat and mass flux } \\
\text { sedimentation/erosion } \\
\text { climate/sea level }
\end{array}\right.
$$

This tectonic boundary scenario constitutes a continuous infinity of parameters (the value of these parameters over all the surface of the basin, for all times) that are traditionally determined in labor-intensive fashion and subject to individual bias.

\section{Incorrect and/or incomplete models}

\section{Multiphase flow in fractured porous media}

There is a vast amount of experimental data on relative permeabilities and capillary pressure relations (Brooks \& Corey 1964; Lenhard \& Parker 1987; Nordtvedt et al. 1997; Liu et al. 1998). In the absence of experimental data, expressions provided by Brooks \& Corey (1964) and Van Genuchten (1980) are the most commonly used ones (see Lenhard et al. $1989 \mathrm{~b}$ for a comparison). However, because of the sensitivity of parameters to rock texture, and fluid configuration and properties, and hysteresis in the relations, a unified model has not been available. Hysteresis in the relative permeability and capillary pressure relations arise because of the changes in intrapore fluid configuration (Aziz \& Settari 1979; Lenhard et al. 1989a). As the change in fluid configuration cannot be described by the classical multiphase model field variables (saturations and fluid phase composition), additional empirical (and not self-consistently predicted) parameters are introduced to model the observed hysteresis (Parker \& Lenhard 1987; Lenhard et al. 1989a). Progress in this area has been hampered by the absence of a complete set of variables to describe the pore-scale fluid configuration dynamics. Therefore, there is a need for a new multiphase flow formulation that is sufficiently comprehensive to selfconsistently model the changing wetting and other pore scale fluid configurational variations (Ortoleva 1998; Tuncay \& Ortoleva 2001).

\section{Texture evolution models}

Texture sits at the heart of many feedback processes and related coupling that underlie crustal system evolution. In this way, the dependence of many phenomenologic laws on rock texture couples all RTM processes. A complete model of crustal evolution must be based on a sufficiently complete set of textural variables and the equations yielding their dynamics. Including such laws in basin models allows the texture-dependent RTM process rate laws be continuously updated over the evolution period of interest.

We suggest that rigorous models of rock behavior should be of the Markov type - i.e. the rate of change of rock state should only depend on the instantaneous rock state and not on prior history. Stress and strain are related through rock rheology to rock texture $\Theta$ (grain size, shape, packing, mineralogy, and fracture length, aperture and orientation statistics). Pressure solution and grain breakage imply that the rate of change of $\Theta$ depends on stress, denoted $\sigma$. If $\Theta$ satisfies the differential equation $\mathrm{d} \Theta / \mathrm{d} t=G(\Theta, \sigma)$, then, in principle, $\Theta(t)$ is a functional of $\sigma$, i.e. depends on $\sigma\left(t^{\prime}\right)$ for all $t^{\prime}<t$, $\Theta=\Theta[\sigma]$ As rheology depends on $\Theta$, we see that $\Theta[\sigma]$ reflects the entire prior stress history and not just the instantaneous value of $\sigma$. However, this 'memory' in a theory wherein $\Theta$ is not coevolved with $\sigma$ is an artifact of the incompleteness of the model. While there are many stress-strain histories that could lead to the instantaneous state of a rock, only the latter is key to predicting its failure and other behavior (Tuncay et al. 2000a; Tuncay \& Ortoleva 2001).

Weyl (1959) proposed that a texture model was introduced based on a periodic array of truncated spheres. Dewers \& Ortoleva (1990a,b,c, 1991a,b) showed how such a texture model could be used in an RTM model. They described the interplay of effect of diffusion and pressure solution to yield diagenetic bedding, stylolites, and related phenomena (see also Ortoleva 1994a,b, 1998 in the wider context of basin modeling). The limitation of this approach is that, with the exception of single grain plastic deformation (Dewers \& Ortoleva 1991b), it cannot describe mechanical compaction through grain boundary slip or grain breakage. Furthermore, as it is limited to isogeometric deformation, it cannot account for nucleation, a range of particle sizes and the complex interplay between mechanical and pressure solution deformation 
in multimineralic systems. The incompleteness of existing texture models is a major drawback in quantitative basin modeling. Improved models would allow for the discrimination between rocks of similar grain size and porosity but of different origins (e.g. chemically precipitated versus mechanically deposited).

\section{Faulting}

The deformation of brittle rocks is a multiple time and length scale phenomenon. Rocks fail rapidly but heal slowly on geologic time scales (Logan \& Teufel 1986; Fredrich \& Evans 1992). Brittle rocks have two sources of memory. They store elastic energy, and, once failed, have broken grain-grain contacts and gouge that persist over long times. Viscous deformation or failure erase the former while chemical healing processes diminish or erase the latter. When sheared across a large-scale zone, rocks can fail within a meter-scale fault zone. Furthermore, the fault dynamic typically takes the form of a series of short time scale events with long inter-event healing periods to form the faulting cycle. The challenge is to develop a rheologic model of this deformation behavior that captures this multiple scale character autonomously - i.e. from an initially uniform, unfailed system to a faulted one, experiencing intermittent failure-healing cyclicity and complex spatial structure. Therefore, fault simulation must be carried out in three spatial dimensions via a model that incorporates a full suite of crustal RTM processes. A preliminary extension of a basin model to simulate faulting is presented by Ozkan et al. (1998) and Tuncay et al. (2001).

\section{Organic geochemical kinetics}

To attain predictability under a wide range of thermal histories and for kerogen of a variety of compositions, it is necessary to base a maturation model on the detailed chemical reaction network with associated laws guided by rules of organic chemistry. Most existing organic geochemical models are based on overall reactions, which do not capture the intermediate processes and are thereby difficult to calibrate in general (Tissot et al. 1987; Espitalie et al. 1988; Braun \& Burnham 1990; Hunt et al. 1991; Pepper \& Corvi 1995).

\section{INFORMATION THEORY FORMULATION}

A central challenge of basin modeling is to construct the present-day internal configuration and chronology of the subsurface from data of a range of types and quality, sparsely distributed across a study area. As the available data are typically indirect and fraught with uncertainty, an objective methodology is needed that yields the most probable chronology and present-day configuration as well as an estimate of the associated uncertainty. Further, there is often a great quantity of data that are too time consuming to be fully inte- grated by classical methods, and methods of analysis are often subject to individual bias. We now outline the MAI approach to the challenges of petroleum Exploration and Production $(\mathrm{E} \& \mathrm{P})$ based on an information theory (probabilistic) integration of basin modeling and large databases.

Basin data must be integrated with modeling to compensate for the incompleteness of both. Furthermore, the integration must be automated, i.e. seismic, well-log, core and other data must, to the extent possible, be used as direct input to the model so as to reduce labor-intensive tasks and to eliminate bias in the interpretation of the geologic record. On the other hand, the automation should somehow integrate our geologic experience/expertise in a natural way so as to minimize the extent of the computations.

There are two main categories of factors that an automated procedure must determine to run a model. The first is the least well-known parameters in the phenomenologic expressions and, second, the scenario of factors influencing the basin at its boundaries (uplift/subsidence and compression/extension/wrenching; basement heat flux, climate, and sediment/erosion). The latter factors change across the basin's boundaries and over the history of the basin (see Fig. 3). Below we outline a new approach, presenting a derivation of an equation for the most probable history of the spatial distribution of the least well-constrained boundary factors. Having delineated these factors, one may use them with a basin model to estimate the time course of the internal state of a basin from the inception of a basin to the present. This approach has been demonstrated for an engineering problem (Tuncay \& Ortoleva 2002).

Let $B$ represent the time course of a set of influences acting on the basin boundaries (see Figs 2 and 3). Thus, $B$ represents the histories of compression/extension and upheaval/subsidence or of other factors (basement heat and fluid flux, sea level, sediment input, erosion) acting at each point on the basin's top, side, and bottom. Let $\rho[B]$ be the probability of a given scenario $B$ of these influences. For example, if $B$ represents the basement heat flux at all points on the basin

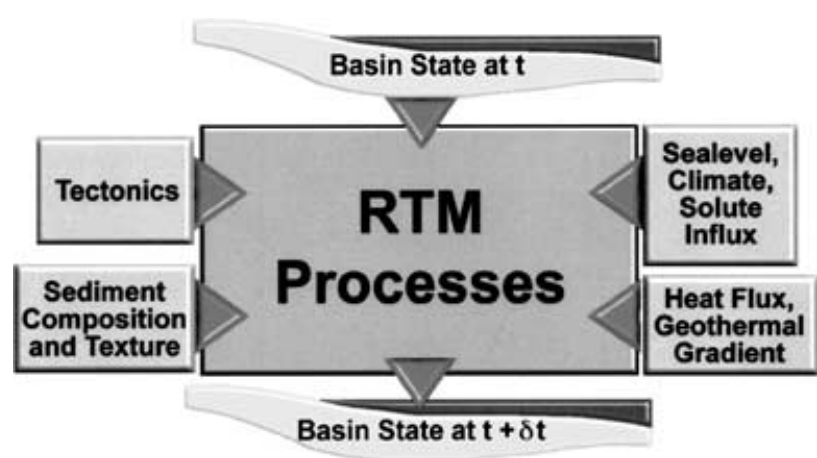

Fig. 3. Schematic flow chart showing how the interplay of geologic data and reaction-transport-mechanical process modules evolve the basin over each computational time interval $\delta t$. 
bottom for all times of the basin's history (inception to present), then $\rho$ depends on the infinity of these heat fluxes (all points on the bottom for all times). Let $S$ indicate an integration of the infinity of such variables, i.e. a functional integral; then normalization of $\rho$ implies

$S_{B} \rho=1$.

Information theory (Jaynes 1957) in the present context is based on the entropy $S$ defined via

$S=-S_{B} \rho \ln \rho$.

$S$ is taken to be a measure of the uncertainty we have in the state of a system; thus as the number of possible states of the system increases so does the entropy. The probability $\rho$ is determined to be that functional of $B$, which maximizes $S$ subject to equation 3 and information we may have about the system (e.g. seismic, well-log, fluid pressure, core analysis, etc.).

Let $O^{(k)}$ be the $k$ th set of the aforementioned data we have on the basin $k=1,2, \ldots, N_{\text {error }}$. Thus, $O^{(1)}$ could be a seismic survey on one area, $O^{(2)}$ a survey on another, $O^{(3)}$ is a suite of logs at various locations, etc. Similarly, let $\Omega^{(k)}$ be the synthetic seismic or other data as constructed using a basin simulator. With this, we construct the $k$ th error $E^{(k)}$ defined such that

$E^{(k)}=\sum_{j=1}^{N^{(k)}}\left(\Omega_{j}{ }^{(k)}[B]-O_{j}^{(k)}\right)^{2}$

As a basin simulation depends on $B$, then so does the synthetic data $\Omega^{(k)}$ constructed from it. In equation (5), $N^{(k)}$ is the number of data values of type $k, O_{j}{ }^{(k)}$ is the $j$ th value of type $k\left(j=1,2, \ldots, N^{(k)}\right)$ and $\Omega^{(k)}$ is the synthetic value of $O_{j}{ }^{(k)}$. With this, we impose the conditions

$S_{B} \rho E^{k}=E^{(k)^{*}}$

where $E^{(k)^{*}}$ is an estimated value of $E^{(k)}$ obtained from our general knowledge of the accuracy of the available data, numerical accuracy of the basin simulator, and the formulas used to construct $\Omega^{(k)}$ from it.

The spatial sparseness of the available data does not allow us to determine the spatial dependence of $B$ on short length scales. Furthermore, practical basin simulation does not allow for very fine grid spacing. Thus, it is not feasible to seek a very fine spatial scale resolution of the $B$ parameters. A similar consideration holds for the time dependence of the $B$ parameters. To constrain the scale at which we wish to delineate the space-time variations in the state of the system, we impose the conditions

$S_{B} \rho \int_{0}^{t_{\text {present }}} \mathrm{d} t \frac{1}{A} \int_{\text {boundary }} \mathrm{d}^{2} r \frac{1}{2}\left|(\underline{\nabla}-\underline{n}(\underline{n} \cdot \underline{\nabla})) B_{\alpha}\right|^{2}=\chi_{\alpha}$

where $t_{\text {present }}$ is the age of the basin ( $t=0$ being the time at basin inception), and $n$ is a unit normal to the basin's bound- ary pointing outward; $\underline{n}$ and $\nabla$ terms imply a tangential gradient. Similarly,

$S_{B} \rho \int_{0}^{t_{\text {present }}} \mathrm{d} t \frac{1}{A} \int_{\text {boundary }} \mathrm{d}^{2} r \frac{1}{2}\left(\frac{\partial B}{\partial t}\right)^{2}=\theta_{\alpha}$

In the above, $B_{\alpha}$ is the $\alpha$ th of the $N_{\mathrm{b}}$ boundary factors ( $\left.B=\left\{B_{1}, B_{2}, \ldots, B_{\mathrm{N}_{\mathrm{b}}}\right\}\right)$ and $\chi_{\alpha}$ and $\theta_{\alpha}$ are estimates that constrain the spatial and time derivatives of $B_{\alpha}$ while $A$ is the (time-dependent) surface area of the basin. The types of boundary factors we shall consider are $\alpha=$ basement heat flux, lateral boundary shape for compression/extension tectonics, shape of the bottom of the basin.

In the light of information theory (Jaynes 1957), we maximize $S$ subject to the constraints (equations 3, 6-8) to obtain $\rho$, we find,

$$
\begin{aligned}
\ln \rho= & -\ln Q-\sum_{k=1}^{N_{\text {error }}} \beta_{k} E^{(k)} \\
& -\sum_{\alpha=1}^{N_{b}} \lambda_{\alpha} \int_{0}^{t_{\text {present }}} \mathrm{d} t \frac{1}{A} \int_{\text {boundary }} \mathrm{d}^{2} r\left|(\underline{\nabla}-\underline{n}(\underline{n} \cdot \underline{\nabla})) B_{\alpha}\right|^{2} \\
& -\sum_{\alpha=1}^{N_{b}} \omega_{\alpha} \int_{0}^{t_{\text {present }}} \mathrm{d} t \frac{1}{A} \int_{\text {boundary }} \mathrm{d}^{2} r\left(\frac{\partial B_{\alpha}}{\partial t}\right)^{2} .
\end{aligned}
$$

This completes the formal construction of $\rho$ (once the normalization constant $Q$ is evaluated via equation (3) and the Lagrange multipliers $(\beta-, \lambda$-, $\omega$-parameters $)$ are fixed via equations (6-8).

The most probable space-time dependence of the $B_{\alpha}$ are determined to be those which maximize $\rho$. We find, upon setting the functional derivatives of equation (9) to zero,

$\frac{\delta \ln \rho}{\delta B_{\alpha}}=0\left(\alpha=1,2, \cdots, N_{\mathrm{b}}\right)$

To find $B$, we shall solve this set of functional differential equations.

Our approach can be more explicitly illustrated for the most probable history of the basement heat flux. Let $B(x, y$, $t)$ be the vertical heat flux into the bottom of a basin at map view position $(x, y)$ at time $t$. For the simple case of a single type of error $E$, we have

$\beta \frac{\delta E}{\delta B(x, y, t)}-\frac{\chi}{A}\left[\frac{\partial^{2} B}{\partial x^{2}}+\frac{\partial^{2} B}{\partial y^{2}}\right]-\frac{\theta}{A} \frac{\partial^{2} B}{\partial t^{2}}=0$

for area $A$ of the basin bottom taken to be constant for simplicity of illustration here. This equation has the character of a space-time diffusional dynamic interacting with the nonlinear $\delta E / \delta B$ term that is a functional derivative of $E$ with respect to $B(x, y, t)$. The $\delta E / \delta B$ term is constructed using a basin simulator.

In the approach presented above, two conditions (equations 7 and 8 ) are used to constrain the spatial and temporal resolution of the $B$ parameters. The use of prior information to regularize the inverse problems is a common practice. This 
can be achieved through an additional error measure in the form of

$E_{\alpha}{ }^{B}=\sum_{j=1}^{M}\left(B_{\alpha}{ }^{j}-B_{\alpha}{ }^{j p}\right)^{2}$

where $M$ is the number of points used to discretize $B$, and $B_{\alpha}{ }^{j p}$ is the prior information, which is usually taken as the initial guess. The use of prior information is convenient. However, it may also be misleading if it masks the observed data. An alternative approach to account for prior information is through the use of the minimum relative entropy principle (Kullback 1959; Kapur 1988; Woodbury \& Ulrych 1996). The minimum relative entropy principle suggests that the probability $\rho$ is constructed by the minimization of the functional $H$ :

$H=S_{B} \rho \ln \frac{\rho}{q}$

with respect to $\rho$. In equation (13), $q$ is the a priori probability distribution. The use of a quadratic error measure (such as equation 12) for the set of uncertain parameters corresponds to the assumption of a Gaussian distribution for the prior information. The minimum relative entropy principle allows one to tailor the a priori probability to the degree of uncertainty once has accumulated through the experience/expertise.

An approximation to $\rho$ can be obtained by expanding equation (9) around the most probable $B$

$$
\begin{aligned}
\left.\ln \rho \approx \ln \rho\right|_{\underline{B}^{m}} & +\left.\sum_{\alpha=1}^{N_{\mathrm{b}}} \sum_{i=1}^{n} \frac{\partial \ln \rho}{\partial B_{\alpha}{ }^{i}}\right|_{\underline{B}^{m}} \Delta B_{\alpha}{ }^{i} \\
& +\left.\sum_{\alpha=1}^{N_{\mathrm{b}}} \sum_{i, j=1}^{n} \frac{1}{2} \frac{\partial \ln \rho}{\partial B_{\alpha}{ }^{i} \partial B_{\alpha}{ }^{j}}\right|_{\underline{B}^{m}} \Delta B_{\alpha}{ }^{i} \Delta B_{\alpha}{ }^{j}+\cdots
\end{aligned}
$$

At the most probable scenario $\left(\underline{B}^{m}\right)$ the second term vanishes. This approximate probability distribution accounts for all the available information, i.e. geologic data and associated error, a priori information, and regularization constraints for temporal and spatial distributions. The matrix of the quadratic term is constructed in the course of carrying out a large number of basin simulations to solve equation (10) numerically. Equation (14) is used to assess the uncertainty associated with the $B$ parameters.

A flowchart for the information theory approach is shown in Fig. 4. Tuncay \& Ortoleva (2002) presented preliminary results in the context of reservoir modeling using this approach.

\section{CONCLUSIONS}

The development of basin simulators has been influenced by several factors:

- availability of mathematical models to describe individual RTM processes;

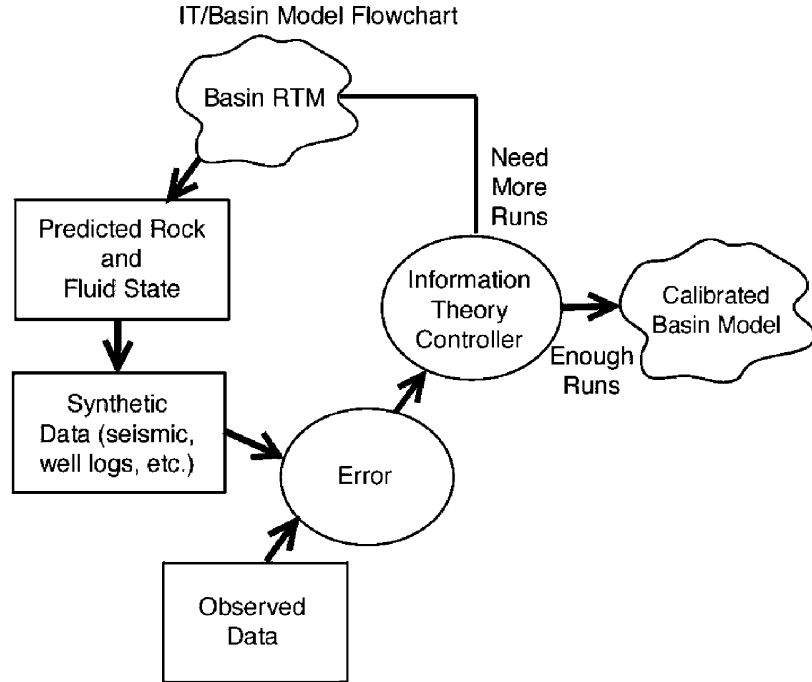

Fig. 4. This automated methodology is based on computational algorithm and I/O to yield the most probable state of the system given observed data and comprehensive physics and chemistry in the basin simulator.

- improvements in computer hardware;

- availability of efficient, stable and accurate numerical techniques; and

- interest and support by industry and government agencies. While applied E\&P teams have always been pressing for a basin simulator that can run on aC in a short time, the reality is that accounting for the many RTM processes requires supercomputing power. While many research teams have attempted to develop simplified models, they have usually failed to communicate the likely pitfalls of an incomplete model. What needed are collaborative projects between industry, government, and academia that combine long-time scientific and financial commitments to make developments of comprehensive basin simulators feasible.

A serious limitation to the successful use of comprehensive basin simulators is the large amount of CPU time presently required for each simulation. This CPU time depends on the spatial dimensionality, spatial resolution, and the number of processes accounted for. The time/cost of the labor-intensive development of basin input data has also been a prohibiting factor in making comprehensive models effective, as has the difficulty in calibrating some of the phenomenologic parameters. Likely availability of faster CPUs and massive parallel architecture and algorithms as well as automated input data schemes based on information theory should accelerate these computations in the next 5 years.

Quantitative basin modeling provides several approaches to the goal of establishing steady and long-term petroleum through exploration and production technologies that utilize the computer-automated analysis of well-log, seismic, geochemical, and other data. Notable among the benefits of this MAI technology are the following: 
- Improve the prediction of reservoir location and characteristics (fracture and matrix permeability, reserves in place, reservoir geometry, in situ stress, etc.) to lower the cost of exploration and production for deep reserves.

- Identify compartments and, thereby, locate by-passed resources.

- Make use of the billions of dollars of well-log, seismic, core, geochemical, and other basin data, which are presently under-used because of the cost of labor-intensive and often biased methods for interpretation.

- Use MAI-predicted reservoir characteristics to plan directions for horizontal wells and other factors to optimize field development and production.

- Achieve quantitative assessment of risk/uncertainty using an MAI approach.

At present, the total investment in basin modeling is a negligible percentage of even the most conservative estimates of the benefits that the comprehensive, MAI basin-modeling technology would yield. We conclude that more resources should be made available for the development of comprehensive basin simulators and MAI software in the near future so as to make the benefits of this technology available to the industry.

\section{ACKNOWLEDGEMENTS}

This work was supported by a grant from the Office of Science of the United States Department of Energy (grant \# DE-FG02-91ERl4175); and a contract \# DE-RA2699FT40160 from the United States Department of Energy.

\section{REFERENCES}

Al-Shaieb Z, Puckette J, Abdalla A, Ely P (1994) Three levels of compartmentation within the overpressured interval of the Anadarko Basin. In: Basin Compartments and Seals (ed. Ortoleva P), pp. 69-84. AAPG, Tulsa, Oklahoma, AAPG Memoir no. 61.

Aziz K, Settari A (1979) Petroleum Reservoir Simulation. Applied Science Publishers, London.

Berkowitz B (1995) Analysis of fracture network connectivity using percolation theory. Mathematical Geology, 27, 467-83.

Berryman JG (1980) Confirmation of Biot's theory. Applied Physics Letters, 37, 383-4.

Berryman JG (1986) Effective medium approximation for elastic constants of porous solids with microscopic heterogeneity. Journal of Applied Physics, 59, 1136-40.

Berryman JG (1992) Single-scattering approximations for coefficients in Biot's equations of poroelasticity. Journal of Acoustic Society of America, 91, 551-71.

Bour O, Lerche I (1994) Numerical modelling of abnormal fluid pressures in the Navarin Basin, Bering Sea. Marine and Petroleum Geology, 11, 491-500.

Bradley JS, Powley D (1994) Pressure compartments in sedimentary basins: a review. In: Basin Compartments and Seals (ed. Ortoleva P), pp. 3-26, AAPG, Tulsa, Oklahoma, AAPG Memoir no. 61.

Braun RL, Burnham AK (1990) Mathematical model of oil generation, degradation, and expulsion. Energy and Fuels, 4, 132-46.
Bredehoeft JD, Wesley JB, Fouch TD (1994) Simulations of the origin of fluid pressure, fracture generation, and the movement of fluids in the Uinta Basin. Utah AAPG Bulletin, 1994, 1729-47.

Brooks RH, Corey AT (1964) Hydraulic Properties of Porous Media. Hydrology Paper no. 3. Colorado State University, Fort Collins.

Burrus J, Osadetz K, Wolf S, Doligez B, Visser K, Dearborn D (1996) A two-dimensional regional basin model of Willston basin hydrocarbon systems. AAPG Bulletin, 80, 265-91.

Celia MA, Binning P (1992) A mass conservative numerical solution for two-phase flow in porous media with application to unsaturated flow. Water Resources Research, 28, 2819-28.

Chen Y, Chen W, Park A, Ortoleva P (1994) Role of pressuresensitive reactions in seal formation and healing: application of the CIRF. A reaction-transport code. In: Basin Compartments and Seals (ed. Ortoleva P), pp. 403-16. AAPG, Tulsa, Oklahoma, AAPG Memoir no. 61.

Cobbold PR (ed.) (1993) New insights into salt tectonics: collection of invited papers reflecting the recent developments in the field of salt tectonics. Tectonophysics 228 .

Currie JB, Nwachukwu SO (1974) Evidence on incipient fracture porosity in reservoir rocks at depth. Bulletin of Canadian Petroleum Geology, 22, 42-58.

Daudre B, Cloetingh S (1994) Numerical modeling of salt diapirism: influence of the tectonic regime. Tectonophysics, 240, 59-79.

Dewers T, Ortoleva P (1990a) A coupled reaction/transport/ mechanical model for intergranular pressure solution, stylolites, and differential compaction and cementation in clean sandstones. Geochemica et Cosmochemica Acta, 54, 1609-25.

Dewers T, Ortoleva P (1990b) Geochemical self-organization. Part III. A mechano-chemical model of metamorphic differentiation. American Journal of Science, 290, 473-521.

Dewers T, Ortoleva P (1990c) Force of crystallization during the growth of siliceous concretions. Geology, 18, 204-7.

Dewers T, Ortoleva P (1991a) Interaction of reaction, mass transport, and rock deformation during diagenesis: mathematical modeling of intergranular pressure solution, stylolites, and differential compaction and cementation. In: Prediction of Reservoir Quality Through Chemical Modeling, Vol. 49. (eds Meshri I, Ortoleva P), pp. 147-60. American Association of Petroleum Geologists Memoir, Tulsa, OK.

Dewers T, Ortoleva P (1991b) Influences of clay minerals on sandstone cementation and pressure solution. Geology, 19, 1045-8.

Dewers T, Ortoleva P (1994a) Nonlinear dynamical aspects of basin hydrology: fluid compartment formation and episodic fluid release. American Journal of Science, 294, 713-55.

Dewers TA, Ortoleva P (1994b) Formation of stylolites, marl/ limestone alternations, and clay seams accompanying chemical compaction of argillaceous carbonates In: Diagenesis. Part IV. Developments in Sedimentology (eds Chilingarian GV, Wolf $\mathrm{KH}$ ), pp. 155-216. Elsevier, Amsterdam.

Dickey PA, Cox WC (1977) Oil and gas in reservoirs with subnormal pressure. AAPG Bulletin, 61, 2134-42.

Engelder T (1987) Joints and shear fractures in rock. In: Fracture Mechanics of Rocks (ed. Atkinson BK), pp. 27-69. Academic Press, Orlando.

Engelder T, Geiser P (1980) On the use of regional joint sets as trajectories of paleostress fields during the development of the Appalachian Plateau, New York. Journal of Geophysical Research, $\mathbf{8 5}, 6319-41$.

Espitalie J, Ungerer P, Irwin I, Marquis F (1988) Primary cracking of kerogens: experimenting and modeling $\mathrm{C} 1, \mathrm{C} 2-\mathrm{C} 5, \mathrm{C} 6-\mathrm{C} 15$, and C15+ classes of hydrocarbons formed. Organic Geochemistry, 13, 893-9. 
Fischer MP, Gross MR, Engelder T, Greenfield RJ (1995) Finite element analysis of the stress distribution around a pressurized crack in layered elastic medium-implications for the spacing of fluid-driven joints in bedded sedimentary-rock. Tectonophysics, 247, 49-64.

Forbes PL, Ungerer P, Mudford BS (1992) A two dimensional model of overpressure development and gas accumulation in Venture Field, Eastern Canada. AAPG Bulletin, 76, 318-38.

Forster A, Merrian DF, Hoth P (1998) Geohistory and thermal maturation in the Cherokee Basin (Multicontinent USA), AAPG Bulletin, 82 (9), 1673-93.

Fredrich JT, Evans B (1992) Strength recovery along simulated faults by solution transfer processes. In: 33rd US Rock Mechanics Symposium (eds Tillerson JR, Warersik WR), pp. 121-30. Balkema, Rotterdam.

Friedman M (1976) Fracture in rock. Reviews of Geophysics and Space Physics, 13, 352-8, 383-9.

Ghaith A, Chen W, Ortoleva P (1990) Oscillatory methane release from shale source rock. Earth Science Reviews, 29, 241-8.

Gordon DS, Flemings PB (1998) Generation of overpressure and compaction-driven fluid flow in a Plio-Pleistocene growth-faulted basin, Eugene Island 330, offshore Louisiana. Basin Research, 10, 177-96.

Gross MR (1993) The origin and spacing of cross joints - examples from the Monterey formation, Santa-Barbara coastline, California. Journal of Structural Geology, 15, 737-51.

Hadad A, Bensabat J, Rubin H (1996) Simulation of immiscible multiphase flow in porous media: a focus on the capillary fringe of oil-contaminated aquifers. Transport in Porous Media, 12, 24569.

Hancock PL, Al Kadhi A, Walper JL (1984) Regional joint sets in the Arabian platform as indicators of intraplate processes. Tectonophysics, 3, 27-43.

Harris JF, Taylor GL, Walper JL (1960) Relation of deformational fractures in sedimentary rocks to regional and local structures. AAPG Bulletin, 44, 1853-73.

Hunt JM, Lewan MD, Hennet RJC (1991) Modeling oil generation with time-temperature index graphs based on the arrhenius equation. AAPG Bulletin, 75, 795-807.

Huyakorn PS, Panday S, Wu YS (1994) A three-dimensional multiphase flow for assessing NAPL contamination in porous and fractured media. Part 1. Formulation. Journal of Contaminant Hydrology, 16, 109-30.

Jackson MPA, Talbot CJ (1986) External shapes, strain rates, and dynamics of salt structures. Geological Society of America Bulletin, 97, 305-23.

Jaynes ET (1957) Information theory and statistical mechanics. Physical Review, 106, 620-30.

Kaluarachchi JJ, Parker JC (1989) An efficient finite element method for modeling multiphase flow. Water Resource Research, 25, 43-54

Kapur JN (1988) Maximum Entropy Models in Science and Engineering. John Wiley, New York.

Kipp KL, Russell TF, Otto JS (1992) A new renumbering for iterative solution of ground-water flow and solute transport equations. In: Computational Methods in Water Resources IX: Numerical Methods in Water Resources (eds Russell TF, Ewing RE, Brebbia CA, Gray WG, Pinder GF), pp. 495-502. Computational Mechanics Publications, Boston, MA.

Koen AD (1993) Subsalt play could rekindle outlook for Gulf of Mexico. Oility and Gas Journal, 91, 23-6.

Koudina N, Garcia RG, Thovert J-F, Adler PM (1998) Permeability of three-dimensional fracture networks. Physical Review E, 57, 4466-79.
Kulander BR, Barton CC, Dean SL (1979) The Application of Fractography to Core and Outcrop Fracture Investigations, p. 174. US Department of Energy, Morgantown Energy Technology Center, Report METC/SP-79/3.

Kullback S (1959) Information Theory and Statistics. John Wiley, New York.

Lee M-K, Williams DD (2000) Paleohydrology of the Delaware Basin, Western Texas: overpressure development, hydrocarbon migration and ore genesis. AAPG Bulletin, 84, 961-74.

Lenhard RJ, Parker JC (1987) Measurement and prediction of saturation-pressure relationships in three phase porous media systems. Journal of Contaminant Hydrology, 1, 407-24.

Lenhard RJ, Parker JC, Kaluarachchi JJ (1989a) A model for hysteretic constitutive relations governing multiphase flow. Part 3. Refinements and numerical simulations. Water Resources Research, 25, 1727-36.

Lenhard RJ, Parker JC, Mishra S (1989b) On the correspondence between Brooks-Corey and van Genuchten models. Journal of Irrigation and Drainage Engineering, 110, 744-51.

Lerche I (1991) Inversion of dynamical indicators in quantitative basin analysis models. Part I. Theoretical considerations. Mathematical Geology, 23, 817-32.

Liu YP, Hopmans JW, Grismer ME, Chen JY (1998) Direct estimation of air-oil and oil-water capillary pressure and permeability relations from multi-step outflow experiments. Journal of Contaminant Hydrology, 32, 223-45.

Logan JM, Teufel LW. The effect of normal stress on the real area of contact during frictional sliding of rocks. Pure and Applied Geophysics, 124, 471-86.

Long JCS, Billaux DM (1987) From field theory to fracture network modeling: an example incorporating spatial structure. Water Resources Research, 23, 1201-16.

Lorenz JC, Teufel LW, Warpinski NR (1991) Regional fractures. Part I. A mechanism for the formation of regional fractures at depth in flat-lying reservoirs. AAPG Bulletin, 75, 1714-37.

Luo X, Vasseur G (1995) Modelling of pore pressure evolution associated with sedimentation and uplift in sedimentary basins. Basin Research, 7, 35-52.

Luo X, Vasseur G (1996) Geopressuring mechanism of organic matter cracking: numerical modeling. AAPG Bulletin, 80, 856-73.

Luo X, Vasseur G, Pouya A, Lamoureux-Var V, Poliakov A (1998) Elastoplastic deformation of porous medium applied to the modeling of compaction at basin scale. Marine and Petroleum Geology, 15, 145-62.

Mallory WW (1977) Oil and gas from fractured shale reservoirs in Colorado and northwest New Mexico. Rocky Mountain Association of Geologists Special Publication, 1, 38.

Maubeuge F, Lerche I (1993) A north Indonesian basin: geo, thermal and hydrocarbon generation histories. Marine and Petroleum Geology, 10, 231-45.

Maubeuge F, Lerche I (1994) Geopressure evolution and hydrocarbon generation in a north Indonesian basin: two-dimensional quantitative modelling. Marine and Petroleum Geology, 104, 104-15.

Maxwell JM, Ortoleva P (1994) Simulating the development of a three-dimensional basinwide overpressured compartment. In: Basin Compartments and Seals (ed. Ortoleva P), pp. 429-34. AAPG Memoir 61, Tulsa, OK.

Mazariegos R, Andrews MJ, Russell JE (1996) Modeling the evolution of salt structures using nonlinear rocksalt flow laws. Tectonophysics, 256, 129-43.

McPherson BJOL, Bredehoeft JD (2001) Overpressures in the Uinta Basin, Utah: analysis using a three-dimensional basin evolution model. Water Resources Research, 37, 857-71. 
McPherson BJOL, Garven G (1999) Hydrodynamics and overpressure mechanisms in the Sacremento Basin, California. American Journal of Science, 299, 429-66.

Mello UT, Karner GD (1996) Development of sediment overpressure and its effect on thermal maturation: application to the Gulf of Mexico Basin. AAPG Bulletin, 80, 1367-96.

Nickelsen RP, Hough VND (1967) Jointing in the Appalachian Plateau of Pennsylvania. Geological Society of America Bulletin, 78, 609-30.

Nicolis C, Nicolis G (eds) (1987) Irreversible Phenomena and Dynamical Systems Analysis in Geosciences. D. Reidel Publishing Co., Dordrecht, Holland.

Nicolis G, Prigogine I (1977) Self-Organization in Non-Equilibrium Systems: From Dissipative Structures to Order Through Fluctuations. Wiley-Interscience, New York.

Nordtvedt JE, Ebeltoft E, Iversen JE, Sylte A, Urkedal H, Vatne KO, Watson AT (1997) Determination of three-phase relative permeabilities from displacement experiments. SPE Formation Evaluation, 12, 221-6.

O'Brien JJ, Lerche I (1986) The preservation of primary porosity through hydrocarbon entrapment during burial. Formation Evaluation, Society of Petroleum Engineers. Vol. 1. pp. 295-9.

Oda M (1986) An equivalent continuum model for coupled stress and fluid flow analysis in jointed rock masses. Water Resources Research, 22, 1845-56.

Odling NE (1992) Network properties of a two-dimensional natural fracture pattern. Pure and Applied Geophysics, 138, 95-114.

Ortoleva P (1994a) Geochemical Self-Organization. Oxford University Press, New York.

Ortoleva P (ed.) (1994b) Basin Compartments and Seals. American Association of Petroleum Geologists, Tulsa, OK, AAPG Memoir 61.

Ortoleva P (1998) Basin Compartment Fundamentals. Topical Report (Project no. GRI-97/0097). Gas Research Institute, Chicago.

Ortoleva P, Merino E, Chadam J, Moore CH (1987a) Geochemical self-organization. Part I. Reaction-transport feedback mechanisms and modeling approach. American Journal of Science, 287, 979-1007.

Ortoleva P, Merino E, Moore CH, Chadam J (1987b) Geochemical self-organization. Part II. The reactive-infiltration instability. American Journal of Science, 287, 1008-40.

Ortoleva P, Al-Shaieb Z, Puckette J (1995) Genesis and dynamics of basin compartments and seals. American Journal of Science, 295, $345-427$.

Ortoleva P, Hallet B, McBirney A, Meshri I, Reeder R, Williams P (eds) (1990) Self-organization in geological systems. Proceedings of a Workshop Held 26-30 June 1988, University of California at Santa Barbara. Earth Science Reviews 29.

Ozkan G, Tuncay K, Ortoleva P (1998) Process-based fault seal/ conduit prediction. In: 1998 AAPG Annual Convention Abstracts (CD-ROM Format). AAPG, Salt Lake City, UT (May 17-28).

Panday S, Wu YS, Huyakorn PS, Springer EP (1994) A threedimensional multiphase flow model for assessing NAPL contamination in porous and fractured media. Part 2. Porous medium simulation examples. Journal of Contaminant Hydrology, 16, 131-56.

Panday S, Forsyth PA, Falta RW, Wu YS, Huyakorn PS (1995) Considerations for robust compositional simulations of surface nonaqueous phase liquid contamination and remediation. Water Resources Research, 31, 1273-89.

Parker JC, Lenhard RJ (1987) A model for hysteretic constitutive relations governing multiphase flow. Part 1. Saturation-pressure relations. Water Resources Research, 23, 2187-96.
Payne DF, Ortoleva PJ (2002a) A model for lignin alteration, Part I. A kinetic reaction-network model. Organic Geochemistry, 32 (9), 1087-1101.

Payne DF, Ortoleva PJ (2002b) A model for lignin alteration. Part II. Numerical model of natural gas generation and application to the Piceance Basin, Western Colorado. Organic Geochemistry, 32 (9), 1073-85.

Payne DF, Tuncay K, Park A, Comer J, Ortoleva P (2000) A reaction-transport-mechanical approach to modelling the interrelationships between gas generation, overpressuring, and fracturing - implications for the Upper Cretaceous natural gas reservoirs of the Piceance Basin, Colorado. AAPG Bulletin, 84, 545-65.

Pepper AS, Corvi PJ (1995) Simple kinetic-models of petroleum formation. Part 1. Oil and gas generation from kerogen. Marine and Petroleum Geology, 12, 291-319.

Person M, Garven G (1992) Hydrologic constraints on petroleum generation within continental rift basins: theory and application to the Rhine Graben. AAPG Bulletin, 76, 468-88.

Person M, Toupin D, Eadington P (1995) One-dimensional models of groundwater flow, sediment thermal history and petroleum generation within continental rift basins. Basin Research, 7, 81-96.

Person M, Raffensperger JP, Ge S, Garven G (1996) Basin-scale hydrogeologic modeling. Reviews of Geophysics, 34, 61-87.

Peters A (1992) CG-like algorithms for linear systems stemming from the FE discretization of the advection-dispersion equation. In: Computational Methods in Water Resources IX: Numerical Methods in Water Resources (eds Russell TF, Ewing RE, Brebbia CA, Gray WG, Pinder GF), pp. 511-20. Computational Mechanics Publications, Boston, MA.

Pollard DD, Aydin A (1988) Progress in understanding jointing over the past century. Geological Society of America Bulletin, 100, 1181-204.

Polyansky OP, Poort J (2000) 2D modeling of fluid flow and heat transport during the evolution of the Baikal rift. Journal of Geochemical Exploration, 69, 77-81.

Powley D (1980) Normal and abnormal pressure. Lecture presented to AAPG Advanced Exploration Schools, 1980-1987.

Powley D (1990) Pressures, hydrogeology, and large scale seals in petroleum basins. In: Self-Organization in Geological Systems, Proceedings of a Workshop Held 26-30 June 1988 (eds Ortoleva P, Hallet B, McBirney A, Meshri I, Reeder R, Williams P). Elsevier, New York.

Qin C, Ortoleva P (1994) Banded diagenetic pressure seals: types, mechanisms and homogenized basin dynamics. In: Basin Compartments and Seals (ed. Ortoleva P), pp. 385-400. AAPG, Tulsa, OK.

Quandalle P, Sabathier JC (1989) Typical features of a multipurpose reservoir simulator. SPE Reservoir Engineering, 4, 475-80.

Renard F, Gratier JP, Brosse E (1999a) The different processes involved in the mechanism of pressure solution in quartz-rich rocks and their interactions. In: Quartz Cement in Oil Field Sandstones (eds Worden R, Morad S). International Association of Sedimentologists Special Publication, pp. 67-78. Blackwell Science, Oxford, UK.

Renard F, Park A, Ortoleva P, Gratier JP (1999b) A transitional pressure solution model. Tectonophysics, 312, 97-115.

Roberts SJ, Nunn JA (1995) Episodic fluid expulsion from geopressured sediments. Marine and Petroleum Geology, 12, 195-204.

Schegg R, Cornford C, Leu W (1999) Migration and accumulation of hydrocarbons in the Swiss Molasse Basin: implications of a 2D basin modeling study. Marine and Petroleum Geology, 16, 511-31. 
Schneider F, Potdevin JL, Wolf S, Faille I (1996) Mechanical and chemical compaction model for sedimentary basin simulators. Tectonophysics, 263, 307-17.

Schultz-Ela DD, Jackson MPA, Vendeville BC (1994) Mechanics of Active Diapirism. Bureau of Economic Geology, Report of Investigation no. 224. Austin, TX.

Segall P, Pollard DD (1983) Joint formation in granitic rock of the Sierra Nevada. Geological Society of America, 94, 563-75.

Seni SJ, Jackson MPA (1984) Sedimentary Record of Cretaceous and Tertiary Salt Movement, East Texas Basin. Bureau of Economic Geology, Report of Investigations no. 139. Austin, TX.

Sleep BE, Sykes JF (1993a) Compositional simulation of groundwater contamination by organic compounds. Part 1. Model development and verification. Water Resources Research, 29, 1697-708.

Sleep BE, Sykes JF (1993b) Compositional simulation of groundwater contamination by organic compounds. Part 2. Model applications. Water Resources Research, 29, 1709-18.

Stover CS, Ge S, Weimer P, McBride B (2001) The effects of salt evolution, structural development, and fault propagation on Late Mesozoic/Cenozoic oil migration: a two-dimensional fluid flow study along a megaregional profile in the northern Gulf of Mexico. Bulletin of American Association of Petroleum Geologists, 85, 1945-66.

Suetnova E, Vasseur G (2000) 1-D modelling rock compaction in sedimentary basins using a visco-elastic rheology. Earth and Planetary Science Letters, 178, 373-83.

Sukirman Y, Lewis RW (1993) A finite element solution of a fully coupled implicit formulation for reservoir simulation. International Journal for Numerical and Analytical Methods in Geomechanics, 17, 677-98.

Tissot BP, Pelet R, Ungerer P (1987) Thermal history of sedimentary basins, maturation indices, and kinetics of oil and gas generation. AAPG Bulletin, 71, 1445-66.

Tuncay K, Ortoleva P (2001) Salt tectonics as a self-organizing process: a three dimensional reaction, transport and mechanics model. Journal of Geophysical Research, 106, 803-18.

Tuncay K, Ortoleva P (2002) Probability functionals, homogenization and comprehensive reservoir simulators. In: Resource Recovery, Confinement, and Remediation of Environmental Hazards, Institute of Mathematics and its Applications, Vol. 131. (eds Chadam J, Cunningham A, Ewing RE, Ortoleva P, Wheeler MF), pp. 161-78. Springer-Verlag, New York.

Tuncay K, Park A, Ortoleva P (2000a) Sedimentary basin deformation: an incremental stress rheology approach. Tectonophysics, 323, 77-104.

Tuncay K, Park A, Ortoleva P (2000b) A forward model of three dimensional fracture orientation and characteristics. Journal of Geophysical Research, 105, 16719-35.

Tuncay K, Khalil A, Ortoleva P (2001) Failure, memory and cyclic fault movement. Bulletin of Seismological Society of America, 91, $538-52$.
Turcotte DL (1992) Fractals, chaos, self-organized criticality and tectonics. Terra Nova, 4, 4-12.

Ungerer P, Burrus J, Doligez B, Chenet PY, Bessis F (1990) Basin evaluation by integrated two-dimensional modeling of heat transfer, fluid flow, hydrocarbon generation, and migration. AAPG Bulletin, 74, 309-35.

Van Genuchten MT (1980) A closed-form equation for predicting the hydraulic conductivity of unsaturated soils. Soil Science Society of the American Journal, 44, 892-8.

Van Keken PE, Spiers CJ, van den Berg AP, Muyzert EJ (1993) The effective viscosity of rocksalt: implementation of steady-state creep laws in numerical models of salt diapirism. Tectonophysics, 225, $457-76$

Wang C, Xie X (1998) Hydrofracturing and episodic fluid flow in shale-rich basins - a numerical study. AAPG Bulletin, 82, 1857-69.

Watts JW (1986) A compositional formulation of the pressure and saturation equations. SPE Reservoir Engineering, 1, 243-52.

Wendebourg J (2000) Modeling multi-component petroleum fluid migration in sedimentary basins. Journal of Geochemical Exploration, 69, 651-6.

Weyl PK (1959) Pressure solution and the force of crystallization - a phenomenological theory. Journal of Geophysical Research, 64, 2001-25.

White SP (1995) Multiphase nonisothermal transport of systems of reacting chemicals. Water Resources Research, 31, 1761-72.

Wieck J, Person M, Strayer L (1995) A finite element method for simulating fault block motion and hydrothermal fluid flow within rifting basins. Water Resources Research, 31, 3241-58.

Wilson HH (1977) 'Frozen-in' hydrocarbon accumulations or diagenetic traps - exploration targets. AAPG Bulletin, 61, 483-91.

Woodbury AD, Ulrych TL (1996) Minimum relative entropy inversion: theory and application to recovering the release history of a groundwater contaminant. Water Resources Research, 32, 2671-81.

Wu HQ, Pollard DD (1995) An experimental study of the relationship between joint spacing and layer thickness. Journal of Structural Geology, 17, 887-905.

Young LC, Stephenson RE (1983) A generalized compositional approach for reservoir simulation. SPE Journal, 21, 727-41.

Yu Z, Lerche I (1995) Three-phase fluid migration with solubilities in a two-dimensional basin simulation model. Marine and Petroleum Geology, 12, 3-16.

Yu Z, Lerche I, Bour Q (1995) Inversion of dynamical indicators in quantitative basin analysis models. Part III. Multiwell information and two-dimensional case histories. Mathematical Geology, 27, 41-68.

Zhao K, Lerche I (1993) Inversion of dynamical indicators in quantitative basin analysis models. Part II. Synthetic tests and a case history using dynamical indicator tomography. Mathematical Geology, 25, 107-23. 


\section{Appendix D}

Sheen, D-H., C-E. Baag, K. Tuncay, and P. Ortoleva 2003. Parallel simulation of wave propagation in three dimensional poroelastic media, AGU Annual Meeting (Received the best student poster award in the seismology section). 


\section{Parallel Simulation of Wave Propagation in Three-Dimensional Poroelastic Media}

Dong-Hoon Sheen ${ }^{1,2 *}$, Chang-Eob Baag ${ }^{1}$, Kagan Tuncay ${ }^{2} a n d$ Peter J. Ortoleva2.

${ }^{1}$ School of Earth and Environmental Sciences, Seoul National University, Seoul, Korea

2 Laboratory for Computational Geodynamics, Indiana University, Bloomington, IN, USA

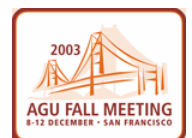

\section{Abstract}

Parallelized velocity-stress staggered-grid finite-difference method to simulate wave propagation in 3-D heterogeneous poroelastic media is presented. Biot's poroelasticity theory is used to study the behavior of wavefield in fluid saturated media. In the poroelasticity theory, fluid velocities and pressure are included as descriptive field variables to those for the pure elasticity to account for the interaction between pore fluid and solid matrix. Discretization of governing equations for finite-difference approximation is performed for a total of 13 field variables in 3-D Cartesian coordinates: six components for velocity, six components for solid stress, and fluid pressure. The scheme has fourth-order accuracy in space and second-order accuracy in time. Also, to simulate wave propagation in an unbounded medium, the perfectly matched layer (PML) method is used as an absorbing boundary condition. In contrast with the pure elastic problem, the larger number of field variables requires a huge sum of core memory inevitably. Hence, a realistic simulation can hardly be run on serial platforms. Therefore, the computationally efficient scheme to run on large parallel environments is required. The parallel implementation is achieved by using a spatial decomposition and the portable message passing interface (MPI). Direct comparisons are made for serial and parallel computations. The efficiency of parallelization for the poroelastic wave modeling are demonstrated using model examples.

\section{Introduction}

- Numerical Simulation of wave propagation in fluid saturated poroelastic media

- Account for pore fluid properties such as fluid density, bulk modulus, viscosity and saturation.

- Based on Biot's theory (1962).

- Staggered-grid finite-difference method to increase the accuracy of the numerical simulation ( $4^{\text {th }}$ order accuracy in space and $2^{\text {nd }}$ order accuracy in time domain)

- Parallelization via Message Passing Interface (MPI).

- Absorbing boundary condition to simulate wave propagation in an unbounded medium

- Perfectly Matched Layer (PML) method (Berenger, 1994).

- The method of complex coordinates (Chew and Weedon, 1994) to formulate the velocity-stress staggered-grid formulation.

\section{Theory}

- Biot's equations (1962) for a fluid-saturated, statistically isotropic, locally homogeneous, poroelastic medium

$\rho \ddot{\mathbf{u}}+\rho_{f} \ddot{\mathbf{w}}-\left(\lambda_{c}+\mu\right) \nabla \nabla \cdot \mathbf{u}-\mu \nabla^{2} \mathbf{u}-\alpha M \nabla \nabla \cdot \mathbf{w}=0$

$\rho_{f} \ddot{\mathbf{u}}+m \ddot{\mathbf{w}}+b \dot{\mathbf{w}}-\alpha M \nabla \nabla \cdot \mathbf{u}-M \nabla \nabla \cdot \mathbf{w}=0$

where

u: displacement vector for the solid;

w: displacement vector of the fluid relative to that for the solid;

$\rho$ : the overall density of the saturated medium determined

by $\phi \rho_{f}+(1-\phi) \rho_{s}$;

$\rho_{f}$ : density of the fluid;

$\rho_{s}:$ density of the solid;

$\phi$ : porosity;

$\lambda_{c}$ : Lamé constant of the saturated matrix;

$\mu$ : shear modulus of the dry porous matrix;

$m$ : effective fluid density;

$\eta$ : viscosity of the fluid;

$K$ : permeability of the porous medium;

$b$ : mobility of the fluid defined by $\eta / K$;

$K_{s}$ : bulk modulus of the solid;

$K_{f}$ : bulk modulus of the fluid;

$K_{b}$ : bulk modulus of the dry porous matrix;

$\alpha=1-K_{b} / K_{s}$;

$M=\left[f / K_{f}+(\alpha-\phi) / K_{s}\right]-1$.

\section{Finite-difference formulation}

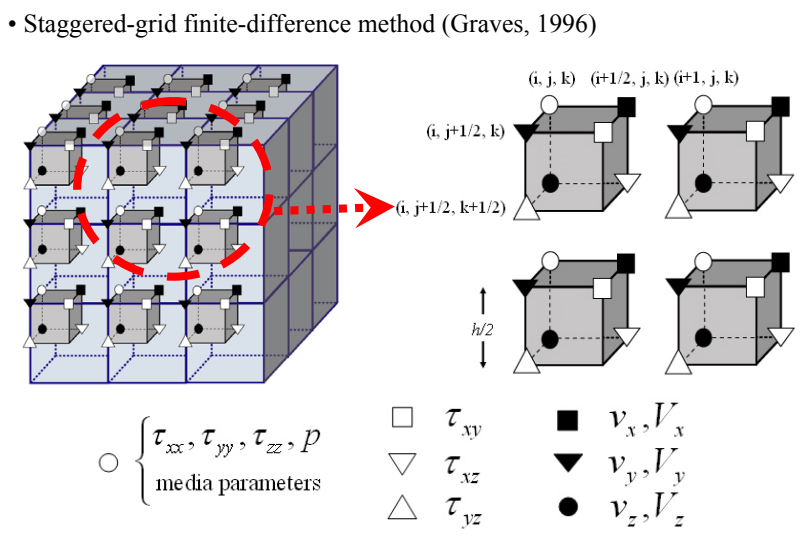

\section{Absorbing boundary condition}

- Using the concept of complex coordinates (Chew and Weedon, 1994) in the frequency domain with a time dependence of $e^{-i \omega t}$, the complex coordinate stretching variables can be written as

$\tilde{x}_{i}=\int_{0}^{x_{i}} s_{i}\left(x_{i}^{\prime}\right) d x_{i}^{\prime}, s_{i}\left(x_{i}^{\prime}\right)=a_{i}^{s}+i \omega_{i}^{s} / \omega$,

where $a_{i}^{s} \geq 1$ is a scaling factor and $\omega_{i}^{s} \geq 0$ is an attenuation factor

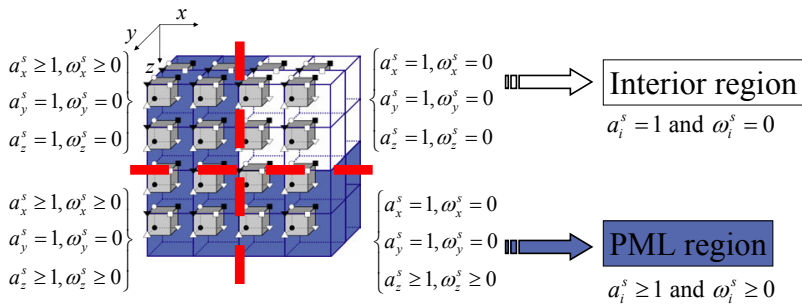

- In this work, the scaling factor and the attenuation factor have the following forms

$$
\begin{aligned}
& a_{i}^{s}=1+a_{\text {max }}\left(l_{i} / L_{\mathbf{P M L}}\right)^{n}, \\
& \omega_{i}^{s}=2 \pi f_{0} \omega_{\text {max }}\left(l_{i} / L_{\mathbf{P M L}}\right)^{n+\alpha},
\end{aligned}
$$

where $l_{i}$ is the distance from the interface between the PML region and the interior region, $L_{\mathrm{PML}}$ is the thickness of the PML region, $a_{\max }$ and $\omega_{\max }$ are empirical coefficients and $f_{0}$ is the dominant frequency of the source. 


\section{Parallelization}

- Poroelastic wave propagation needs 4 more wavefield components and 5 more material properties than elastic wave propagation. In the PML region, it requires 21 more wavefield components than that of elastic wave propagation.

- For parallelization, the 3-D computational domain is decomposed into relatively small sub domains.
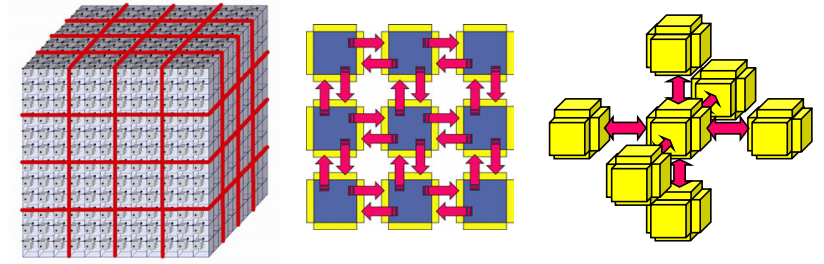

- The velocity components $\left(v_{i}, V_{i}\right)$ and the stress $\left(\tau_{i j}, p\right)$ components are staggered in spatial and time domain. Therefore, no stress component can be updated, before the velocity update is completed and vice versa.

- Each processor updates wavefield components within its domain. After each processor has updated its domain, the processors exchange the wavefield information at the edges of sub domains. For communication at the internal edges a ghost layer whose thickness is 2 grid spacing has been introduced. The length of this ghost layer is half of the length of spatial finite difference operator.

- The exchange of the wavefield information in PML region is the same as in interior (Non-PML) region.

\section{Numerical examples}

\section{- Homogeneous model}

- We studied wave propagation in homogeneous poroelastic media to examin the accuracy of the FD and PML method.

- Gas saturated sandstone

- Ideal inviscid pore fluid

$\cdot 160 \times 160 \times 160$ grids

- 10 grids of PML on all sides of the computational boundary

- A point source with a dominant frequency of $15 \mathrm{~Hz}$

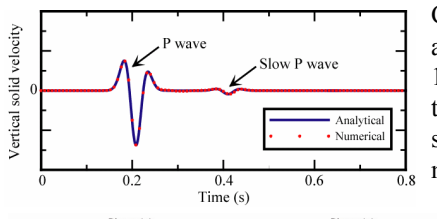

Comparison of numerical and analytical waveforms (Dai et al., 1995) of the vertical component of the solid velocity for an explosive source in an infinite homogeneous medium.
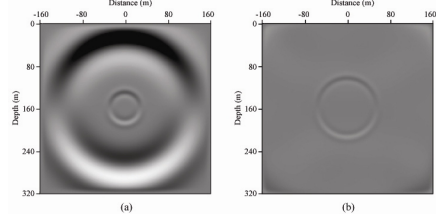

Snapshots of the vertical component of the solid velocity at $t=0.24 \mathrm{~s}$ (a) and $t=0.32 \mathrm{~s}$ (b).

\section{- Anticline model}

-We simulated an anticline model to illustrate poroelastic wave propagation in 3-D heterogeneous media.

-The saturated anticline sandstone is enclosed by water saturated shale.

- $500 \times 300$ × 200 grids

- The spatial / time step: $0.4 \mathrm{~m} / 0.08 \mathrm{~ms}$

-A point source (dominant frequency of $150 \mathrm{~Hz}$ )
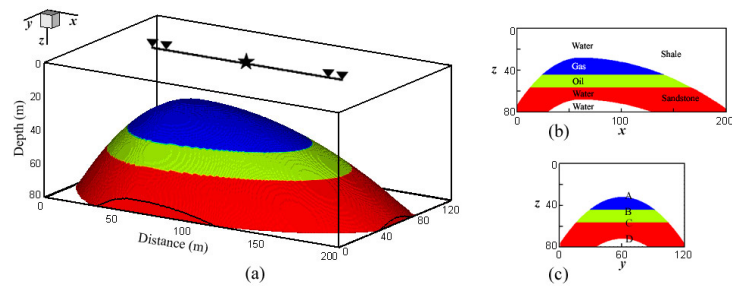

Geometry of the 3-D anticline model and source/receiver configuration. (a) Source and receivers are located at surface. Star and triangles denote the seismic source and receivers, respectively. 80 receivers with $2 \mathrm{~m}$ spacing are used for recording synthetic seismogram. (b) A saturated anticline sandstone reservoir enclosed by water saturated shale. (c) Interfaces are labeled A, B, C, and D.

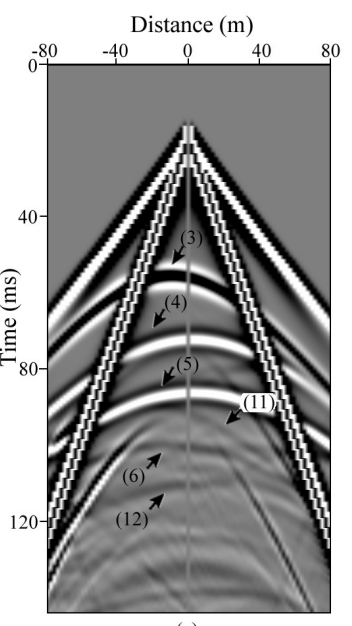

(a)

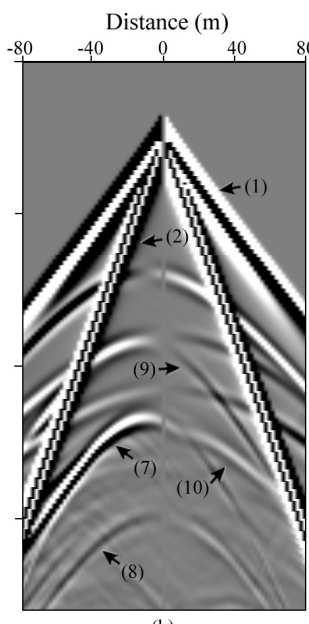

(b)
Synthetic seismograms from the vertical component (a) and horizontal component (b) of the solid velocity at the free surface for the 3-D anticline model shown in Figure 10. Labeling of arrivals are indicated for (1) direct $P$ wave, (2) Rayleigh wave, (3) P wave reflection from interface A, (4) P wave reflection from interface $\mathrm{B},(5) \mathrm{P}$ wave reflection from interface $\mathrm{C},(6)$ $\mathrm{P}$ wave reflection from interface $\mathrm{D}$, (7) $\mathrm{S}$ wave reflection from interface $\mathrm{A}$, (8) S wave reflection from interface B, (9) PS reflection from interface A, (10) PS reflection from interface B, and (11) and (12) P wave multiple reflections.

\section{- Parallelization}
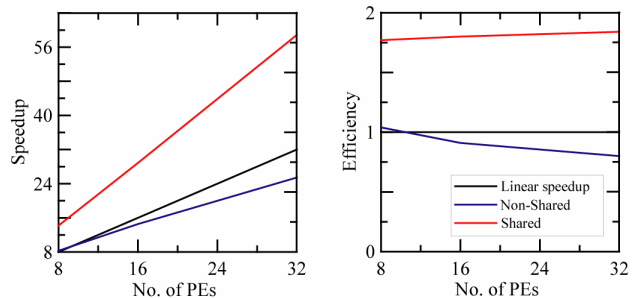

Observed speedup (serial execution time / parallel execution time) for $\mathrm{N}=200^{3}$ and 600 iterations.

Black lines indicate linear speedup.

Blue lines resulted from non-shared memory passing for intra-node communication

Red lines using shared-memory passing for intra-node communication (2 Processing Elements (PEs) per Node).

\begin{tabular}{|c|c|c|c|c|c|c|c|c|}
\hline & \multicolumn{3}{|c|}{ Non-Shared Memory (sec) } & \multicolumn{4}{c|}{ Shared Memory (sec) } \\
\hline No. of PEs & 1 & 8 & 16 & 32 & 1 & 8 & 16 & 32 \\
\hline $\begin{array}{c}\text { Computation } \\
\text { Time }\left(\mathrm{T}_{1}\right)\end{array}$ & 45648.47 & - & - & - & 45648.47 & - & - & - \\
\hline $\begin{array}{c}\text { Computation } \\
\text { Time }\left(\mathrm{T}_{\mathrm{n}}\right)\end{array}$ & - & 5503.50 & 3137.88 & 1793.54 & - & 3218.34 & 1580.91 & 775.56 \\
\hline $\begin{array}{c}\text { Speed-up } \\
\left(\mathrm{T}_{1} / \mathrm{T}_{\mathrm{n}}\right)\end{array}$ & - & 8.29 & 14.55 & 25.45 & - & 14.18 & 28.87 & 58.86 \\
\hline $\begin{array}{c}\text { Efficiency } \\
\left(\mathrm{T}_{1} / \mathrm{T}_{\mathrm{n}} / \mathrm{n}\right)\end{array}$ & - & 1.04 & 0.91 & 0.80 & - & 1.77 & 1.80 & 1.84 \\
\hline
\end{tabular}

\section{Conclusion}

To simulate more realistic seismic wave propagation, one should consider the effects of inelasticity, anisotropy and etc. In this work, parallelized velocitystress staggered-grid FD method is developed to simulate wave propagation in 3-D heterogeneous poroelastic media. It is shown that using shared-memory passing for intra-node communication can improve the efficiency of the parallelization. As poroelastic wave propagation simulation requires more memory space and computational efficiency than elastic wave propagation, the parallelization is indispensable for realistic seismic wave propagation simulation.

\section{Acknowledgement}

This work was partially supported by the BK21 program through the School of Earth and Environmental Sciences, Seoul National University, a grant from the Office of Science (\# DE-F602 91ER14175) and a contract from the Office of Fossil Energy (\# DE-RA26-99FT40160) of the United States Department of Energy.

\section{References}

Berenger, J. P., 1994, A perfectly matched layer for the absorption of electromagnetic waves, J. Comput Phys., 114, 185-200,

Biot, M. A., 1962, Mechanics deformation and acoustic propagation in porous media, J. Appl. Phys., 33, 1482-1498.

Chew, W. C., and Weedon, W. H., 1994, A 3-D perfectly matched medium from modified Maxwell's equations with stretched coordinates, Micro. Opt. Tech. Lett., 7. 599-604.

Dai, N., Vafidis, A., and Kanasewich, E. R., 1995, Wave propagation in heterogeneous, porous media: A velocity-stress, finite-difference method, Geophysics, 60, 327-340,

Graves, R. W., 1996, Simulating seismic wave propagation in 3D elastic media using staggered-grid finite differences, Bull. Seism. Soc. Am., 86, 1091-1106. 